\title{
Femtosekunden Photolyse von Diiodmethan in überkritischen Fluiden: Konkurrenz zwischen Photodissoziation und Photoisomerisierung
}

\author{
Dissertation \\ zur Erlangung des Doktorgrades \\ der Mathematisch-Naturwissenschaftlichen Fakultäten \\ der Georg-August-Universität zu Göttingen
}

vorgelegt von

Christian Grimm

aus Göttingen

Göttingen 2003 
D7

Referent: Prof. Dr. J. Troe

Korreferent: Prof. Dr. D. Schwarzer

Tag der mündlichen Prüfung: 05.11.2003 
Für Diana 



\section{Danksagung}

Herrn Prof. J. Troe danke ich für die Anregung und die stete Förderung dieser Arbeit. Besonders das internationale wissenschaftliche Umfeld und die hervorragende Arbeitsatmosphäre in seiner Abteilung haben sehr zum Gelingen dieser Arbeit beigetragen. Auch die Möglichkeit an zahlreichen internationalen Tagungen teilnehmen zu können, hat die Arbeit im Rahmen des von ihm geleiteten Sonderforschungsbereiches 357 sehr gefördert.

Mein ganz besonderer Dank gilt Prof. J. Schroeder für die Betreuung dieser Arbeit. Sein stetes Interesse am Fortgang des Projektes und seine Diskussionsbereitschaft haben diese Arbeit erst ermöglicht. Das Arbeitsklima in seiner Gruppe habe ich immer als Bereicherung empfunden.

Herrn Prof. D. Schwarzer danke ich für die freundliche Übernahme des Korreferates, sowie für seine Diskussionsbereitschaft und seine Hilfe bei der Implementation der Hochdruckmesszelle.

Besonders danke ich auch Herrn Dipl. Chem. Jochen Zerbs für seine unerschütterliche Ausdauer an noch so harten Labortagen und die hervorragende Zusammenarbeit im „Femtoland“. Die gemeinsamen Diskussionen zu aktuellen Themen der Physikalischen Chemie, der Psychologie, der Philosophie und der Önologie haben den Laboralltag ausgesprochen bereichert.

Den Drs. Matthias Kling und Tobias Steinel sei ganz herzlich für die gemeinsamen Laborstunden in frühen „Femtoland“-Tagen und für die hervorragende Korrekturarbeit, auch über den großen Teich hinweg, gedankt.

Auch bei Dr. Thomas Lenzer und Herrn Dipl. Chem. Christian Müller möchte ich mich an dieser Stelle ganz recht herzlich für die fleißige Korrekturarbeit und die hilfreichen Hinweise bedanken.

Meinem Ko-Administrator, Herrn Dipl. Chem. Jörg Hahn, möchte ich an dieser Stelle meinen besonderen Dank für die gemeinsame Lösung aller erdenklichen Computerproblem, nicht nur $\mathrm{T}_{\mathrm{E} X}$ 'nischer Art, aussprechen. 
Für die stets schnelle und kompetente Lösung aller erdenklichen feinmechanischen Probleme und für die Beschreitung des „Inventor"-Neulandes danke ich Herrn Reinhard Bürsing ausdrücklich.

Stellvertretend für alle Mitarbeiter der Werkstätten des Institutes danke ich den Herren Dipl. Ing. Volker Meier und Dipl. Ing. Andreas Knorr für die durchgeführten Arbeiten, die sehr zum Gelingen des Auf- und Ausbaus der Femtosekundenanlage beigetragen haben.

Ganz besonders danke ich auch meinen Eltern, die mich stets in allen Situation unterstützt und gefördert haben.

Meiner Frau Diana danke ich für ihre Unterstützung, ihr Verständnis und ihre Liebe, die das Gelingen dieser Arbeit erst ermöglicht haben. 


\section{Inhaltsverzeichnis}

$\begin{array}{ll}\text { Zusammenfassung } & 1\end{array}$

1 Einleitung 3

2 Das Modellsystem Diiodmethan 9

2.1 Dissoziationsdynamik des isolierten Moleküls . . . . . . . . . . . . . . 9 9

2.2 Einfluss der Lösungsmittelumgebung . . . . . . . . . . . . . . . . . . . 14

2.2.1 Einfluss auf die Molekülschwingungen . . . . . . . . . . . . . . 14

2.2.2 Untersuchungen in der Lösungsmittelmatrix . . . . . . . . . . . . 15

2.2.3 Zeitaufgelöste Untersuchungen . . . . . . . . . . . . . . . . 17

2.2.4 Schwingungsenergietransfer . . . . . . . . . . . . . . . . . . . . . . . . . . . 18

2.3 Bildung des Isomers . . . . . . . . . . . . . . . . . . . . . . . . 18

3 Experimentelle Technik 23

3.1 Wahl der Methode .................... . . 23

3.2 Das Lasersystem im Überblick . . . . . . . . . . . . . . . . . . . . . . 24

3.3 Die Komponenten des Lasersystems . . . . . . . . . . . . . . . . . . . . 25

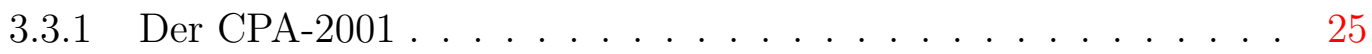

3.3.2 Erzeugung des Pumppulses im NOPA . . . . . . . . . . . . . . 27

3.3.3 Erzeugung des Probepulses im TOPAS . . . . . . . . . . . . 31

3.3.4 Das Pump-Probe-Interferometer . . . . . . . . . . . . . . 32

3.4 Signalaufnahme . . . . . . . . . . . . . . . . . . 34

3.4.1 Messprinzip ........................ 34

3.4 .2 Elektronische Steuerung . . . . . . . . . . . . . 36

3.4 .3 Signalauswertung . . . . . . . . . . . . . . . . . . 37

3.4.4 Charakterisierung des Messsystems . . . . . . . . . . . . . 40

3.5 Aufbau und Funktion der Messzelle . . . . . . . . . . . . . . . . . . . . 42

3.6 Verwendetes Hochdrucksystem . . . . . . . . . . . . . . . . . . . . . 45

3.7 Aufnahme stationärer Absorptionsspektren . . . . . . . . . . . . . . 46

3.8 Verwendete Chemikalien . . . . . . . . . . . . . . . . . 47 
4 Experimentelle Ergebnisse $\quad 49$

4.1 Stationäre Absorptionsmessungen . . . . . . . . . . . . . . . . . . . . . 49

4.1.1 Absorptionsspektren in organischen Lösungsmitteln . . . . . . . 49

4.1.2 Absorptionsspektren in überkritischen Lösungsmitteln . . . . . . 51

4.2 Zeitaufgelöste Absorptionsmessungen . . . . . . . . . . . . . . . 52

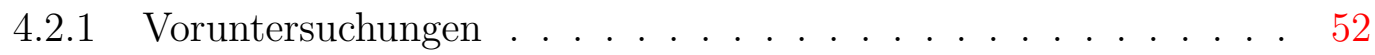

4.2.2 Zeitaufgelöste Messungen in überkritischem $\mathrm{CO}_{2}$. . . . . . . . . 54

4.2.3 Zeitaufgelöste Messungen in überkritischem $\mathrm{CHF}_{3}$. . . . . . . . 72

4.2.4 Zeitaufgelöste Messungen in überkritischem Ethan . . . . . . . . 77

4.2.5 Zeitaufgelöste Messungen in überkritischem Xenon . . . . . . . 82

5 Diskussion $\quad 87$

5.1 Beteiligte Spezies ..................... . . 87

5.1.1 Franck-Condon-Region des angeregten $\mathrm{CH}_{2} \mathrm{I}_{2} \ldots \ldots$. . . . . . 90

5.1 .2 Geminale Rekombination . . . . . . . . . . . . . . . . . 92

5.1 .3 Schnelle Isomerbildung . . . . . . . . . . . . . . . . . . 94

5.1.4 $\mathrm{CH}_{2} \mathrm{I}$-Radikalabsorption .................. 94

5.1.5 Orientierungsrelaxation . . . . . . . . . . . . . . . 96

5.1 .6 Ladungstransferkomplex . . . . . . . . . . . . . . . . . 99

5.1.7 Zusammenfassung . . . . . . . . . . . . . 100

5.2 Einfluss des Schwingungsenergietransfers . . . . . . . . . . . . . . . 101

5.3 Lösungsmitteleinflüsse . . . . . . . . . . . . . . . . . . . . . . . . 104

5.3.1 Ausbeute an Isomer . . . . . . . . . . . . . . . . . . 104

5.3 .2 Isomerisierungsgeschwindigkeit . . . . . . . . . . . . 107

5.3.3 Einfluss der lokalen Dichte . . . . . . . . . . . . . . . . . . . 110

5.4 Mechanismen der Photoisomerisierung . . . . . . . . . . . . . . . 111

5.5 Abschließende Bemerkungen . . . . . . . . . . . . . . . 113

6 Ausblick 115

6.1 Messungen bei niedrigeren Dichten . . . . . . . . . . . . . . . . 115

6.2 Spektrale Entwicklungen . . . . . . . . . . . . . . . 116

6.3 Messungen in der zweiten Absorptionsbande . . . . . . . . . . . . 117

6.4 Zerfallsreaktion des Isomers . . . . . . . . . . . . . . . . . 118

6.5 Messung thermischer Spektren . . . . . . . . . . . . . . . . 118

6.6 Einfluss der lokalen Dichte . . . . . . . . . . . . . . . . . 119

6.7 Untersuchung anderer Dihalomethane . . . . . . . . . . . . . . . . 119

$\begin{array}{ll}\text { Anhang } & 121\end{array}$

A.1 Modellfunktionen des Anpassungsprogramms . . . . . . . . . . . . . . . 121

A.2 Daten der Anpassungen . . . . . . . . . . . . . . . . . . 123

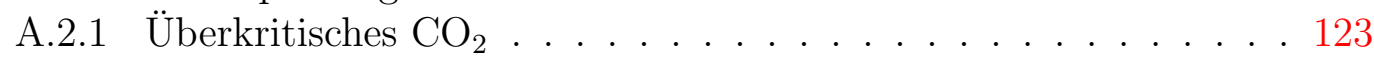

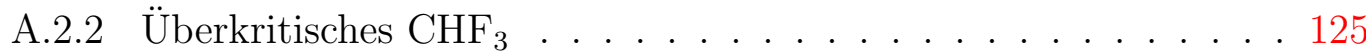


A.2.3 Überkritisches Ethan . . . . . . . . . . . . . . . . . . . . . . . 125

A.3 Thermodynamische Daten . . . . . . . . . . . . . . . 126

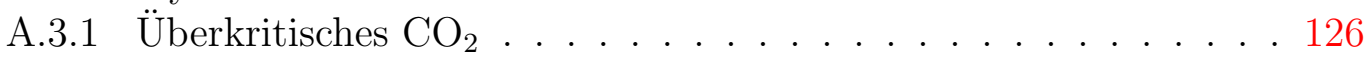

A.3.2 Überkritisches $\mathrm{CHF}_{3}$. . . . . . . . . . . . . . . . . . . . . 128

A.3.3 Überkritisches Ethan . . . . . . . . . . . . . . . . . . . . 130

A.3.4 Überkritisches Xenon . . . . . . . . . . . . . . . . . . . . 131

$\begin{array}{ll}\text { Abbildungsverzeichnis } & 133\end{array}$

$\begin{array}{ll}\text { Tabellenverzeichnis } & 137\end{array}$

$\begin{array}{ll}\text { Literaturverzeichnis } & 139\end{array}$ 



\section{Zusammenfassung}

Die Photodissoziation des Diiodmethans wurde mit der Femtosekunden Pump/ProbeSpektroskopie in überkritischen Fluiden in einem Druckbereich von 100-1000 bar untersucht. Es sollte die in flüssiger Lösung auftretende Konkurrenz zwischen der Dissoziation und der Bildung des iso-Diiodmethans innerhalb eines Lösungsmittelkäfigs nach Photoanregung im Bereich von $300 \mathrm{~nm}$ im Hinblick auf die Struktur und den Einfluss des Lösungsmittelkäfigs genauer untersucht werden. Die aus den Messungen in überkritischen Fluiden erhaltenen transienten Absorptions-Zeit-Profile zeigen eine ähnliche Form, wie die in flüssigen, organischen Lösungsmitteln erhaltenen Messungen. Aus den druckabhängigen Messungen in den verschiedenen Lösungsmitteln zeigt sich sowohl eine Dichteabhängigkeit der Isomerausbeute als auch der Isomerisierungsgeschwindigkeit. Ferner wurden aus den einzelnen Absorptions-Zeitprofilen transiente Spektren rekonstruiert, die deutlich die Charakteristika einer sich abkühlenden Spezies aufweisen, welches der Schwingungsenergierelaxation des gebildeten Isomers zugeordnet wird.

Anhand der experimentellen Daten wurden verschiedene Aspekte des Mechanismus der Photodissoziation und Photoisomerisierung diskutiert. So wurden die zu den Kurzzeitsignalen beitragenden, möglichen Spezies näher betrachtet. Es wurde ein Vergleich verschiedener Lösungsmittel und ihr Einfluss auf die Dissoziation und Isomerisierung vorgenommen. Die Untersuchung der Isomerausbeute zeigt, dass die Ausbeute für alle Lösungsmittel nahezu linear mit der reduzierten Dichte ansteigt, was den Schluss zulässt, dass es sich um einen rein kinematischen Austritt der Fragmente aus dem Lösungsmittelkäfig handelt. Für den nahezu exponentiellen Anstieg der Geschwindigkeitskonstanten der Isomerisierung mit der reduzierten Dichte wurden im Rahmen dieser Arbeit einige Erklärungsansätze diskutiert, z. B. der Einfluss des Schwingungsenergietransfers, die Beeinflussung einer möglichen Reaktionsbarriere durch das Lösungsmittel oder die Auswirkungen der lokalen Dichte. Auf Grundlage dieser Überlegungen wurde für die Photodissoziation des Diiodmethans in Lösung ein kinetisches Modell vorgeschlagen. Zusätzlich wurden aus den spektralen Daten qualitative Aussagen über die Lösungsmittelabhängigkeit des Schwingungsenergietransfers des Isomers in das umgebende Lösungsmittel gemacht. 



\section{Kapitel 1}

\section{Einleitung}

Für das Verständnis komplexer chemischer Reaktionen ist oft eine detaillierte Kenntnis der beteiligten Elementarreaktionen unabdingbar. Von besonderem Interesse sind dabei unimolekulare Reaktionen, wie beispielsweise Zerfallsreaktionen oder intramolekulare Umlagerungen. Unimolekulare Reaktionsmechanismen sind unter isolierten Bedingungen theoretisch und experimentell gut untersucht und verstanden $[1,2,3]$. Weit weniger Informationen existieren hingegen bezüglich des Einflusses der Lösungsmittelumgebung auf die Mechanismen unimolekularer Reaktionen. Dabei sind gerade Reaktionen in der kondensierten Phase für sehr viele chemisch und auch biologisch relevante Prozese außerordentlich wichtig. Das Lösungsmittel kann beispielsweise Energie aufnehmen bzw. abgeben oder die Reaktionspartner in einem Lösungsmittelkäfig gefangen halten und damit einen erheblichen Einfluss auf den Verlauf der Reaktion

nehmen. Die außerordentliche Relevanz unimolekularer Reaktionen in Lösung hat bereits zu einer Vielzahl von Untersuchungen geführt [4].

Bereits in den 30er Jahren erkannten James Franck und Eugen Rabinowitsch in Göttingen die Wichtigkeit des Einflusses der Lösungsmittel [5] auf photochemische Zerfallsreaktionen [6] und postulierten das Prinzip des photolytischen Käfigeffekts [5]. Danach können die im primären Zerfallsschritt photochemisch gebildeten Fragmente direkt in einem weiteren Elementarschritt wieder rekombinieren (geminale Rekombination). Eine solche Reaktion im Lösungsmittelkäfig ist demnach von der Konkurrenz zwischen geminaler Rekombination und dem Austritt der Fragmente aus dem Lösungsmittelkäfig bestimmt.

In den 50er Jahren gelang es schließlich Noyes und Mitarbeitern am Beispiel der Photodissoziation und Rekombination von molekularem Iod den photolytischen Käfigeffekt zu validieren $[7,8,9,10]$. Dazu untersuchten sie bei verschiedenen Quecksilberlinien das Absorptionsverhalten des Iods in Lösung nach bestimmten Photolysezeiten. Sie stellten fest, dass die Quantenausbeute für den Zerfall von Iod in Iodatome nach Photoanregung von eins in der Gasphase auf nur wenige Prozent in der flüssigen Phase absinkt, was mit geminaler Rekombination erklärt wurde. Ihre Studien zur Lösungsmittel- und 
Wellenlängenabhängigkeit führten zur Beschreibung des Einflusses des Lösungsmittelkäfigs über ein einfaches Diffusionsmodell. Dies beinhaltet die Vorstellung, dass die beiden Iodatome auf Grund ihrer Überschussenergie mit einer gewissen kinetischen Energie aus dem Bindungsbruch hervorgehen. Durch Wechselwirkungen mit dem Lösungsmittel werden sie auf thermische Geschwindigkeiten abgebremst und können sich statistisch und diffusiv in einem homogenen, viskosen Lösungsmittelkontinuum bewegen und gegebenenfalls wieder rekombinieren. Die Konkurrenz zwischen Rekombination und Entfernen der Fragmente voneinander ist danach nur diffusionskontrolliert.

Der von Noyes und Mitarbeitern postulierte Einfluss der Lösungsmittelviskosität veranlasste Luther und Troe [11] zur Untersuchung der Dichteabhängigkeit der Reaktion in überkritischen Lösungsmitteln. Sie fanden dabei schon bei Dichten weit unterhalb von typischen Flüssigkeitsdichten eine Abnahme der Quantenausbeute für den Iodzerfall. Weitere intensive Untersuchungen zur Rekombinationsgeschwindigkeit und der Quantenausbeute mit Nanosekundenzeitauflösung in überkritischen Lösungsmitteln durch Schroeder, Troe und Mitarbeiter $[12,13]$ führten zu einer Verbesserung des Diffusionsmodells von Noyes. Demnach kann die Rekombinationsgeschwindigeit und die Quantenausbeute je nach Dichtebereich von verschiedenen Effekten beeinflusst werden. Bei geringeren Dichten sind die Effekte auf die Rekombinationsgeschwindigkeit durch Cluster- bzw. Intermediatkomplexbildung, bei höheren Dichten durch einen vollständig diffusionskontrollierten Mechanismus beschreibbar. Das Absinken der Quantenausbeute wird bei geringeren Dichten den Löschprozessen durch Cluster- bzw. Intermediatkomplexbildung und bei höheren Dichten durch eine Konkurrenz zwischen Energiertransfer in das Lösungsmittel und diffusivem Verlassen des Käfigs beschrieben. Ein Beitrag zur Abnahme der Quantenausbeute durch direktes, impulsives Verlassen des Käfigs durch die Iodatome nach der Dissoziation ist in ihrem Modell dabei vernachlässigbar.

Zur detaillierten Beschreibung der Dynamik der Dissoziations- und Rekombinationsprozesse im Lösungsmittelkäfig von Iod gab es zahlreiche Untersuchungen mit Pikound Femtosekunden-Zeitauflösung an Clustern, in komprimierten Gasen und flüssigen Lösungsmitteln $[14,15,16,17,18]$, woraus ein viel komplexeres Modell der beteiligten dynamischen Dissoziations- und Rekombinationsmechanismen resultierte. So wurde der Einfluss des prädissoziativen Charakters des nach der Photoanregung erreichten elektronischen Zustandes auf die Dissoziationsdynamik untersucht. Das dynamische Modell beinhaltete insbesondere verschiedene geminale Rekombinationsmechanismen, die auf unterschiedlichen Potentialfächen ablaufen können: einen direkten, schnellen geminalen Rekombinationskanal auf der $\mathrm{I}_{2}$-Grundzustandspotentialfäche, bei der das Rekombinationsprodukt durch Schwingungsenergietransfer stabilisiert wird, sowie einen langsameren Kanal, bei dem das Rekombinationsprodukt zunächst in einem elektronischen Zwischenzustand gefangen wird, aus dem es durch strahlende und nichtstrahlende Übergänge in den Iod-Grundzustand gelangen und dort stabilisiert werden kann. Demgegenüber ist es einem Teil der Fragmente möglich, den Lösungsmittelkäfig 
nach der Dissoziation schnell zu verlassen. Diese können ebenfalls auf einer sehr viel längeren Zeitskala von mehreren zehn Pikosekunden geminal rekombinieren oder rein diffusionskontrolliert getrennt werden, um gegebenenfalls später auf einer Mikrosekundenzeitskala nicht-geminal zu rekombinieren. In diesem detaillierteren Modell wurde demnach, im Gegensatz zu vorangegangenen Arbeiten, dem sofortigen, impulsiven Verlassen des Lösungsmittelkäfigs ein wesentlicher Beitrag zugeschrieben.

Zur Kurzzeitdynamik wurden begleitend zahlreiche theoretische Arbeiten veröffentlicht, die sich mittels klassischer [19,20,21,22,23] und gemischt quanten-klassischer [24, 25, 26], molekulardynamischer Methoden mit der Dynamik der DissoziationsRekombinationsmechanismen beschäftigten. Wie die Simulationen zeigen, spielt die Konkurrenz zwischen direktem, impulsiven Verlassen und geminaler Rekombination im Lösungsmittelkäfig nur innerhalb weniger Pikosekunden eine Rolle, wobei die Dissoziations- und Rekombinationsdynamik durch nicht-thermische, hochenergetische Stöße dominiert wird. Erst später setzt ein nur durch diffusive Bewegungen gesteuerter Prozess ein.

In jüngster Zeit wurden weitere Untersuchungen zur Reaktionsdynamik des IodZerfalls in überkritischen Lösungsmitteln durchgeführt $[27,28]$. Insbesondere Schwarzer und Mitarbeiter konnten mit begleitenden klassischen molekulardynamischen Untersuchungen weitere Erkenntnisse zum Mechanismus der Reaktion liefern [28]. Ihre Messungen und die Simulationen ergaben, dass die Quantenausbeute für die Dissoziation des Iods nahezu linear mit steigender Dichte abnimmt. Dabei zeigte sich insbesondere durch eine Darstellung der Quantenausbeute gegen die reduzierte Dichte und damit gegen die Packungsdichte der Lösungsmittelmoleküle bzw. das freie Volumen der aus der Dissoziation gebildeten Fragmente, dass die Quantenausbeute unabhängig von der Art des Lösungsmittels ist. Diese Ergebnisse und die begleitenden Simulationen geben Anlass zu der Vermutung, dass es sich beim Verlassen der Fragmente aus dem Lösungsmittelkäfig um einen rein kinematischen Effekt handelt [28].

Der photolytische Käfigeffekt am Beispiel eines zweiatomigen Halogenmoleküls gilt daher als sehr gut verstanden. Dies legt eine ebenso systematische Untersuchung polyatomarer Moleküle, insbesondere hinsichtlich des Einflusses der Schwingungsrelaxation auf den photolytischen Käfigeffekt, nahe. Aussichtsreiche Kandidaten für diese Untersuchungen sind die Dihalomethane, insbesonders das Diiodmethan $\mathrm{CH}_{2} \mathrm{I}_{2}$, an dem bereits zahlreiche Untersuchungen durchgeführt wurden.

Diiodmethan zerfällt nach UV-Anregung bei $300 \mathrm{~nm}$ in der Gasphase mit einer Quantenausbeute von eins in ein $\mathrm{CH}_{2}$ I-Radikal und ein Iod-Atom [29,30]. Der Hauptteil der Überschussenergie dieses Zerfalls findet sich als innere Energie des $\mathrm{CH}_{2}$ I-Fragmentes wieder, was auf Ähnlichkeiten zur Dissoziation von triatomaren Spezies wie $\mathrm{NO}_{2}$ und auf einen impulsiven Dissoziationsmechanismus hindeutet [31].

In der kondensierten Phase gibt es in Analogie zum Iodzerfall durch das Vorhandensein eines Lösungsmittelkäfigs zu dem direkten Zerfall konkurrierende Prozesse, 
was zu einer abnehmenden Quantenausbeute für die Dissoziation führt. So wurde zunächst in Argon- und Kohlenwasserstoffmatrizen eingefrorenes $\mathrm{CH}_{2} \mathrm{I}_{2}$ untersucht und die Bildung eines im Lösungsmittelkäfig gebildeten Photoprodukts beobachtet $[32,33]$. Dieses Photoprodukt wurde schließlich als $\mathrm{CH}_{2} \mathrm{I}$-I-Isomer identifiziert [33]. Die Ergebnisse aus zeitaufgelösten Messungen in flüssiger Phase gaben Anlass zu kontroversen Interpretationsansätzen. Harris und Mitarbeiter schrieben ihre Signale der Absorption des $\mathrm{CH}_{2} \mathrm{I}$-Radikals gefolgt von einem Prozess der geminalen Rekombination zum $\mathrm{CH}_{2} \mathrm{I}_{2} \mathrm{zu}$ [34], während Yoshihara et al. die Ausbildung eines Ladungstransferkomplexes diskutierten [35]. Nach weiteren Femtosekunden Pump/Probemessungen von Åkesson und Mitarbeitern wurde für die flüssige Phase ebenfalls die Bildung des $\mathrm{CH}_{2} \mathrm{I}$-I-Isomers als Käfigphotoprodukt vorgeschlagen [36]. Die Bildung dieses Isomers als Käfigphotoprodukt nach der Photodissoziation wurde jüngst durch ramanspektroskopische und quantenchemische Methoden von Phillips et al. gestützt [37]. Eine Isomerisierungsreaktion innerhalb eines Lösungsmittelkäfigs wurde auch schon an anderen Systemen wie OClO oder ICN beobachtet [38,39]. Insbesondere ist dieses Verhalten auch in anderen Halogenmethanen, wie $\mathrm{CHI}_{3}$ [40, 41], $\mathrm{CH}_{2} \mathrm{Br}_{2}$ [42], $\mathrm{CH}_{2} \mathrm{ClI}[43,44]$, $\mathrm{CH}_{2} \mathrm{BrI}[45,46,47]$ und $\mathrm{CHBr}_{2} \mathrm{Cl}$ [48] feststellbar. Das derzeit verwendete dynamische Modell nach der Photodissoziation von Diiodmethan in Lösung geht von einer schnellen Bildung des $\mathrm{CH}_{2} \mathrm{I}$-I-Isomers innerhalb des Lösungsmittelkäfigs gefolgt von Schwingungsrelaxation der gebildeten Spezies aus [36,37]. Eine detailliertere Beschreibung des aktuellen Forschungsstandes ist in Kapitel $2 \mathrm{zu}$ finden.

Bisher ist jedoch nur sehr wenig über die genauen Prozesse bekannt, die zur Absenkung der Dissoziationsquantenausbeute in kondensierter Phase führen. So ist noch nicht geklärt, ob wie beim Iod eine geminale Rekombination der Fragmente im Lösungsmittelkäfig stattfindet. Zusätzlich zu dieser in Analogie zum Iodzerfall aufgeworfenen Fragestellung der Konkurrenz zwischen dem Verlassen des Käfigs und dem Entstehen geminaler Käfigphotoprodukte tritt die Frage des Einflusses des Schwingungsenergietransfers auf die Käfigdynamik auf. Auch das genaue Verzweigungsverhältnis zwischen den Prozessen ist nicht geklärt. Des Weiteren kann das Vorhandensein eines direkten Reaktionspfades vom $\mathrm{CH}_{2} \mathrm{I}_{2}$-Eduktmolekül zum $\mathrm{CH}_{2} \mathrm{I}$-I-Isomer diskutiert werden, der durch den Lösungsmittelkäfig ermöglicht wird. Als fundamentale Frage stellt sich, bei welcher Dichte beim Übergang von der Gasphase zur kondensierten Phase die Quantenausbeute für den direkten Zerfall absinkt und somit die Käfigreaktion zum Isomer einsetzt.

Ziel dieser Arbeit war es, die genannten Fragestellungen aufzugreifen und durch die Untersuchung mit Ultrakurzzeitmethoden den Mechanismus der Photodissoziation und Photoisomerisierung in Lösung zu untersuchen. Dazu wurden Experimente in überkritischen Lösungsmitteln wie $\mathrm{CO}_{2}, \mathrm{CHF}_{3}$, Ethan und Xenon im Bereich von 100 1000 bar durchgeführt, da in überkritischen Lösungsmitteln die Dichte und damit die Eigenschaften des Lösungsmittelkäfigs systematisch variiert werden können. Damit könnte es möglich sein, ähnlich wie bei der Photodissoziation von Iod [13], den Über- 
gang zwischen der Situation in der Gasphase und Situation in der flüssigen Phase zu erfassen.

Im folgenden Kapitel 2 wird ein kurzer Abriss der Erkenntnisse zur Dissoziationsdynamik des Diiodmethans gegeben. Insbesondere wird dabei auf den aktuellen Stand der Forschung zur Photoisomerisierung des Diiodmethans genauer eingegangen. In Kapitel 3 findet sich eine detaillierte Beschreibung des verwendeten experimentellen Aufbaus. Die mit diesem Aufbau gewonnenen Messergebnisse sind in Kapitel 4 dargestellt. Diese werden anschließend in Kapitel 5 diskutiert und bewertet. Abschließend wird in Kapitel 6 ein kurzer Ausblick auf den Fortgang des Projektes sowie auf offene Fragestellungen gegeben. 



\section{Kapitel 2}

\section{Das Modellsystem Diiodmethan}

Diiodmethan $\mathrm{CH}_{2} \mathrm{I}_{2}$ ist in vielerlei Hinsicht von wissenschaftlichem Interesse. Insbesondere die photochemischen Eigenschaften wurden unter verschiedenen Aspekten detailliert untersucht. Dabei waren besonders die photochemischen Reaktionen sehr früh Gegenstand der Forschung [29,49]. Gregory und Style veröffentlichten u. a. ein erstes Gasphasenspektrum des Diiodmethanes bei der Untersuchung seiner Photooxidation sowie einen Mechanismus der primären Photoprozesse [29].

In der Natur spielt $\mathrm{CH}_{2} \mathrm{I}_{2}$ als biogen in den Ozeanen

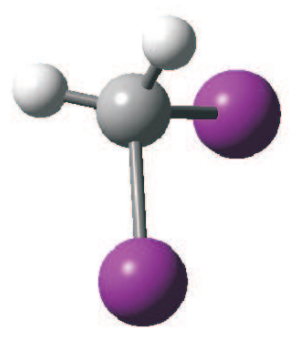
gebildeter Stoff ebenfalls eine große Rolle beim Ozonabbaumechanismus in der Atmosphäre [50,51]. Auch in der organischen Synthese für die photochemische Herstellung von Cyclopropan mit Olefinen, z. B. in der SimmonsSmith-Reaktion [52,53], wird Diiodmethan als Reagenz eingesetzt.

In Elektronenbeugungsuntersuchungen in der Gasphase wurden frühzeitig die strukturellen Daten des Moleküls ermittelt [54]. Danach beträgt der C-I-Abstand 2,21 A, der I-I-Abstand 3,569 $\AA$ sowie der I-C-I-Winkel 114,7.

Im Folgenden soll nun auf die wesentlichen Aspekte der für das Verständis der Isomerisierung wichtigen Photodissoziation des Diiodmethans nach UV-Lichtanregung genauer eingegangen werden.

\subsection{Dissoziationsdynamik des isolierten Moleküls}

Die Dissoziationsdynamik des Diiodmethans wird schon seit geraumer Zeit mit spektroskopischen Methoden untersucht. Das isolierte Molekül liegt in $C_{2 \mathrm{v}}$-Symmetrie vor $[30,55,56]$. Das Schwingungsspektrum wurde z. B. über resonante Raman-Streuung 
oder IR-Spektroskopie ermittelt [57,58] (eine Übersicht über die Schwingungsmoden gibt Tabelle 2.1 auf Seite 14). Die Rotationskonstanten sind aus Millimeterwellenmessungen im Molekularstrahl zu A=22034,437(2) MHz, B=620,584(2) MHz und $\mathrm{C}=605,798(2) \mathrm{MHz}$ bestimmt worden [59]. $\mathrm{CH}_{2} \mathrm{I}_{2}$ ist im Gegensatz zu $\mathrm{CH}_{3} \mathrm{I}$ ein bichromophorisches System [60] und Anregung in den ersten elektronisch angeregten Zustand hat $A_{1} \rightarrow B_{1}$-Charakter. Das Übergangsdipolmoment dieser Anregung ist parallel zur I-I-Verbindungslinie polarisiert [56]. Der Mechanismus der UV-Anregung in den ersten elektronisch angeregten Zustand geht von einer Anregung eines nichtbindenden, am Iod lokalisierten Elektrons in einem Orbital mit p-Charakter in ein antibindendes $\sigma^{*}$-Orbital der C-I-Bindung, also einem $n \rightarrow \sigma^{*}$-Übergang, aus. Das zugehörige Übergangsdipolmoment sollte senkrecht zur C-I-Bindung liegen, jedoch sind im Fall von $\mathrm{CH}_{2} \mathrm{I}_{2}$ starke Kopplungen durch das zweite Iod-Atom anzunehmen [30]. Die weiteren elektronischen Übergänge des Diiodmethans konnten durch Anpassung des stationären Absorptionsspektrums mit mehreren Gaussfunktionen abgeschätzt werden [30,55,61], deren Zentren bei 312, 288, 249 und $212 \mathrm{~nm}$ liegen [62]. Diese Banden entsprechen Übergängen vom elektronischen Grundzustand mit $\widetilde{X}^{1} A_{1}$-Symmetrie in fünf elektronisch angeregte Zustände mit folgenden Symmetrien: $1 B_{1}, 2 B_{1}, B_{2}, 1 A_{1}, 2 A_{1}$ (siehe dazu Abbildung 2.2).

Für die Dissoziation nach UV-Anregung sind die Reaktionskanäle zum $\mathrm{CH}_{2} \mathrm{I}$-Radikal und zwei verschiedenen Spin-Orbit-Zuständen des Iod-Atoms [63,64] sowie zum $\mathrm{CH}_{2^{-}}$ Fragment und molekularem Iod bei bestimmten Anregungswellenlängen energetisch möglich [61]:

$$
\begin{array}{ll}
\mathrm{CH}_{2} \mathrm{I}_{2} \stackrel{h \nu}{\longrightarrow} \mathrm{CH}_{2} \mathrm{I}+\mathrm{I}\left({ }^{2} \mathrm{P}_{3 / 2}\right) & \Delta E^{\circ}=17950 \mathrm{~cm}^{-1}, \\
\mathrm{CH}_{2} \mathrm{I}_{2} \stackrel{h \nu}{\longrightarrow} \mathrm{CH}_{2} \mathrm{I}+\mathrm{I}^{*}\left({ }^{2} \mathrm{P}_{1 / 2}\right) & \Delta E^{\circ}=25510 \mathrm{~cm}^{-1}, \\
\mathrm{CH}_{2} \mathrm{I}_{2} \stackrel{h \nu}{\longrightarrow} \mathrm{CH}_{2}+\mathrm{I}_{2} & \Delta E^{\circ}=30000 \mathrm{~cm}^{-1} .
\end{array}
$$

Kawasaki et al. haben in diesem Zusammenhang die Photodissoziation im Molekularstrahl und die Winkelverteilungen der Photofragmente bestimmt [30]. Aus der Anisotropie der Winkelverteilungen konnte geschlossen werden, dass der Zerfall des Diiodmethans schnell im Vergleich zur Rotationsperiode des Moleküls sein muss. Dies bedeutet, dass der Dissoziationsprozess auf einer sub-ps Zeitskala abläuft. Bei Anregung in das Maximum der ersten Absorptionsbande (siehe Abbildung 2.3) bei $290 \mathrm{~nm}$ $\left(\sim 34500 \mathrm{~cm}^{-1}\right)$ sind aus energetischer Sicht alle drei Reaktionskanäle möglich (siehe Gleichungen R1, R2, R3). Jedoch wurde im Massenspektrum nur eine Linie des I bei $127 \mathrm{amu}$ aber keine Linie des $\mathrm{I}_{2}$ bei $254 \mathrm{amu}$ gefunden. Daher kann davon ausgegangen werden, dass der Reaktionskanal zu molekularem Iod im Bereich der ersten Absorptionsbande nicht zugänglich ist. Dies ist konsistent mit der Annahme, dass die Potentialfläche mit $B_{1}$-Symmetrie aus Symmetriegründen nicht zur Dissoziation von 


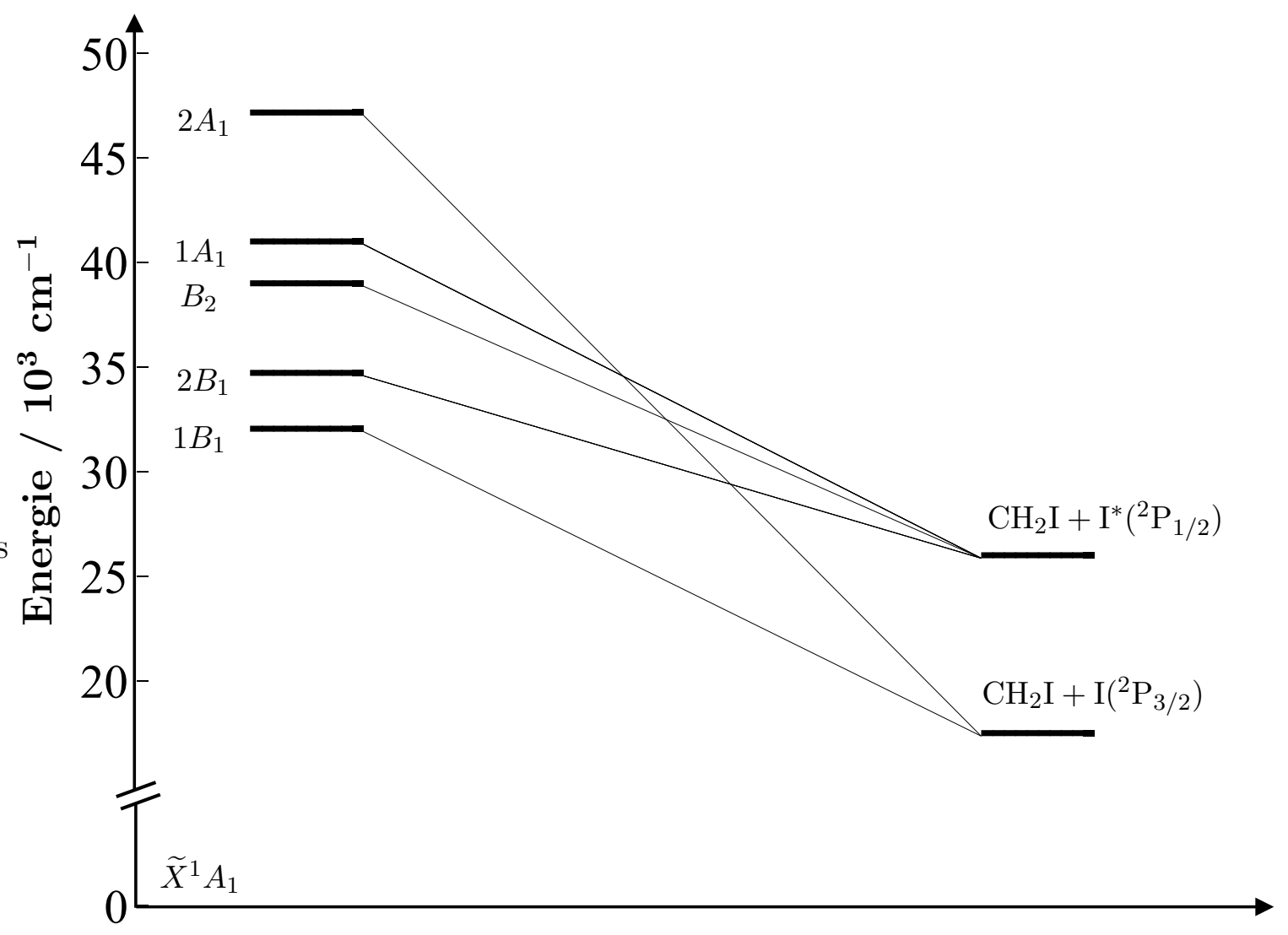

Abstand $\mathrm{CH}_{2} \mathrm{I}-\mathrm{I}$

Abbildung 2.2: Schematische Energiediagramm der niedrigsten elektronischen Zustände des Diiodmethans. Die Korrelation mit den I oder I* Produktkanälen ist ebenfalls dargestellt.

molekularem Iod führen kann $[30,64,65]$. Eine Dissoziation in das Carben-Diradikal und molekulares Iod ist erst mit Anregung unterhalb von $150 \mathrm{~nm}[66,67,68]$, mit Multiphotonenanregung [69] oder mit zeitlich speziell geformten Pulsen (chirped pulses) [70] im Bereich von $300 \mathrm{~nm}$ möglich.

Für die entstehenden Iod-Atome wurde eine Energieabhängigkeit der Quantenausbeuten gefunden $[65,71,72]$. So entsteht unterhalb von $29360 \mathrm{~cm}^{-1}(\sim 340 \mathrm{~nm})$ Anregungsenergie kein angeregtes $I^{*}\left({ }^{2} \mathrm{P}_{1 / 2}\right)$ [65]. Dies führt zu der Annahme, dass aus dem energetisch niedrigsten angeregten Zustand mit $B_{1}$-Symmetrie nur GrundzustandsIod $\left({ }^{2} \mathrm{P}_{3 / 2}\right)$ gebildet wird. Für die UV-Anregung bei $304 \mathrm{~nm}\left(\sim 32900 \mathrm{~cm}^{-1}\right)$ wurde die Quantenausbeute von $I^{*}$ zu 0,25 ermittelt [73].

Als zweites Produkt wird bei der Photodissoziation das $\mathrm{CH}_{2}$ I-Radikal gebildet. In Molekularstrahlexperimenten konnte mit massenspektroskopischen Methoden gezeigt 


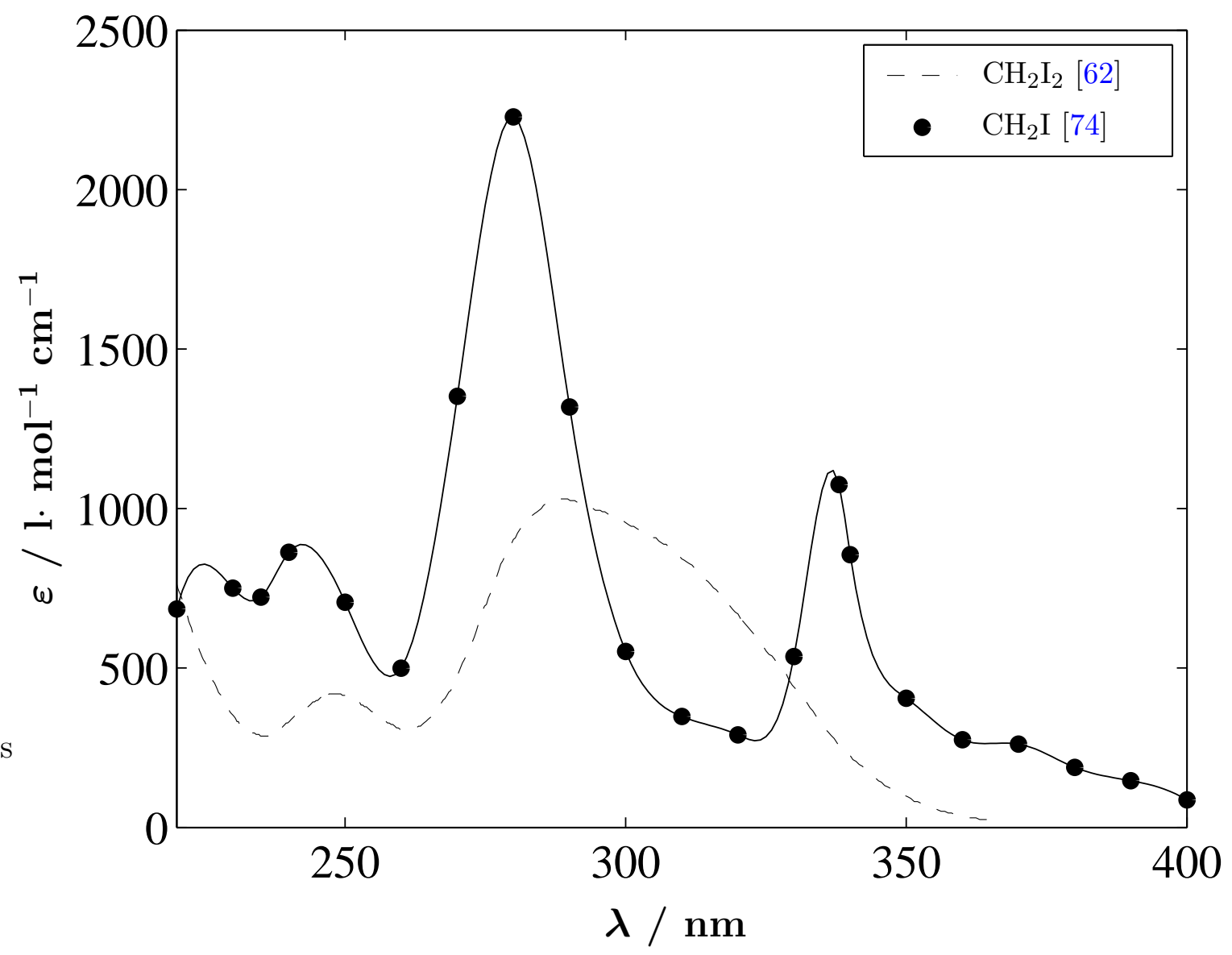

Abbildung 2.3: Vergleich der Absorptionsspektren von $\mathrm{CH}_{2} \mathrm{I}_{2}$ [62] und $\mathrm{CH}_{2} \mathrm{I}$ [74] in der Gasphase.

werden, dass etwa 80-90\% der verfügbaren Energie nach der Dissoziation als rovibronische Energie, mit $40 \%$ rotatorischem und $60 \%$ vibronischem Anteil, im $\mathrm{CH}_{2} \mathrm{I}$ vorliegt [56]. Der vibronische Anteil liegt dabei nahe dem Quasikontinuum der populierten Schwingung [71]. Die hohe Schwingungsanregung ist im Wesentlichen durch das Vorhandensein der sehr niederfrequenten C-I-Schwingung des Radikals begründet, die nur sehr schlecht an die C-H-Moden koppelt. Der restliche Teil der aus der Dissoziation verfügbaren Energie steht dem Iod-Atom in Form von Translationsenergie zur Verfügung. Die gesamte verfügbare Energie $E_{\mathrm{v}}$ kann aus der Beziehung $E_{\mathrm{v}}=h \nu-D_{0}^{0}+E_{\text {in }}^{0}$ abgeschätzt werden [56], wobei $D_{0}^{0}$ die Dissoziationsenergie und $E_{\text {in }}^{0}$ die innere Energie des Moleküls bezeichnet. Diese Werte können mit der Standardbildungsenthalpie abgeschätzt werden: $D_{0}^{0}-E_{\mathrm{in}}^{0} \simeq \Delta H_{298 \mathrm{~K}}^{\mathrm{f}}$. Das $\mathrm{CH}_{2}$ I-Radikal besitzt ebenfalls $C_{2 \mathrm{v}^{-}}$ Symmetrie und sein elektronisches Absorptionsspektrum in der Gasphase verfügt über zwei Maxima bei $280 \mathrm{~nm}$ und $340 \mathrm{~nm}$ [74] (siehe Abbildung 2.3). 
Für die theoretische Beschreibung der Dissoziation wurde die Dynamik der Wellenpaketbewegung im ersten elektronisch angeregten Zustand untersucht. Zunächst wurde dazu ein Exciton-Modell, zweier koppelnder Excitonen der C-I-Bindungen im angeregten Zustand vorgeschlagen [30]. In Messungen der laserinduzierten Fluoreszenz (LIF) durch Zhang et al. [61] bei Anregung von $355 \mathrm{~nm}$ wurden im LIF-Spektrum Beiträge folgender Schwingungen festgestellt: der symmetrischen C-I-Streckschwingung $\nu_{3}$ mit Progression, der asymmetrischen C-I-Streckschwingung $\nu_{9}$ mit erstem Oberton $2 \nu_{9}$ und der I-C-I-Knickschwingung $\nu_{4}$. Die Interpretation der Signale beruht auf der Annahme, dass das im angeregten Zustand geformte Wellenpaket sich hauptsächlich auf der Potentialfläche der symmetrischen C-I-Streckschwingung bewegt, jedoch kann ein Teil des Wellenpakets auch auf der Potentialfläche der asymmetrischen C-IStreckschwingung erzeugt werden. Die Potentialfläche muss dabei jedoch stark repulsiven Charakter haben und damit der dissoziierende Zustand sein, da nur der erste Oberton der $\nu_{9}$-Mode sichtbar ist. Aufgrund des Zusammenspiels zwischen symmetrischer und asymmetrischer Streckschwingung sind die C-I-Bindungen bei der Dissoziation stark verlängert, welches die hohe Schwingungsanregung im Produktradikal erklären würde. Im Vergleich zu Messungen an $\mathrm{CH}_{3} \mathrm{I}$ kann festgestellt werden, dass die Intensität des $2 \nu_{9}$-Obertones im $\mathrm{CH}_{2} \mathrm{I}_{2}$ stärker ausgeprägt ist, was auf eine langsamere Dissoziationsgeschwindigkeit als in $\mathrm{CH}_{3} \mathrm{I}$ hindeutet [61]. Ferner wird durch die Bewegung in der symmetrischen Strecksschwingung der I-C-I-Winkel geändert, so dass ein Beitrag der $\nu_{4}$-Schwingung im LIF-Signal erhalten wird. Die Dissoziation wird dabei als quasidreiatomig angesehen, da keine LIF-Beiträge in $\mathrm{CH}_{2}$-Moden sichtbar und somit als entkoppelt anzunehmen sind. Eine auf diesen Ergebnissen aufbauende quantenmechanische Simulation der Dynamik im ersten angeregten Zustand geht von einem schnellen elektronischen Energietransfer von einem angeregten Iod im Molekül zu dem nichtangeregten Iod aus [60]. Für diesen Energietransfer muss ein nicht-adiabatischer Übergang (surface hopping) zwischen den Potentialflächen (PES, potential energy surface) zweier lokaler C-I-PES stattfinden, die sich entlang der Koordinaten der symmetrischen Streckschwingung kreuzen. Für diesen Übergang werden zwei Möglichkeiten diskutiert: ein diabatisches Limit und ein adiabatisches Limit. Im diabatischen Limit kann das Wellenpaket nachdem es die Kreuzungsregion verlassen hat keinen Übergang zur anderen Potentialfäche finden, so dass ein Energieaustausch sehr langsam wäre. Im adiabatischen Limit hingegen ist die Kopplung beider lokaler PES sehr stark und der Energietransfer läuft sehr schnell ab. Die schnelle Dissoziationsdynamik favorisiert eine Beschreibung im adiabatischen Limit. Aufgrund dieser Struktur der PES wird das nach Anregung in der symmetrischen Streckschwingung befindliche Wellenpaket in zwei Anteile der symmetrischen Streckschwingung aufgeteilt und führt zu einer Bifurkation des Wellenpaketes. Es kehrt nicht mehr in die ursprüngliche Franck-Condon-Region zurück und die Dissoziation kann dann aus einem dieser „Kanäle" stattfinden; eine Abspaltung von $\mathrm{I}_{2}$ ist daher nicht möglich. Dieses theoretische Modell stimmt mit den experimentellen Daten sehr gut überein.

Basierend auf den Untersuchungen von Zhang et al. [61] haben kürzlich Barinovs et al. 
3D-Wellenpaketrechnungen zum $\mathrm{CH}_{2} \mathrm{I}_{2}$ veröffentlicht [75]. Mit ihrer Methode konnten sie u. a. das Ramanspektrum mit sehr guter Übereinstimmung zum Experiment berechnen. Die Zeitskala der Dissoziationsdynamik erstreckt sich in ihren Simulationen ebenfalls über den Femtosekundenzeitbereich.

Während die Dissoziation im $1 B_{1}$-Zustand zu Grundzustands Iod-Atomen führt, werden bei der Anregung in den $2 B_{1}$ sowohl I* als auch I erhalten. Dieses Verhalten wird von $\mathrm{Xu}$ et al. anhand von Ion-Imaging-Messungen [62] mit einer Kreuzung der Potentialflächen (curve crossing), also ebenfalls einem nicht-adiabatischen Übergang, erklärt. Das im $2 B_{1}$-Zustand gebildete Wellenpaket wird danach nahe des Endzustandes in einen Teil aufgeteilt, der auf der $2 B_{1}$ Fläche zu $\mathrm{CH}_{2} \mathrm{I}$ und I* dissoziiert und einen Teil, der nach Kreuzung auf der $2 A_{1}$-Fläche in $\mathrm{CH}_{2} \mathrm{I}$ und I dissoziiert. Solche curve-crossing-Prozesse wurden auch für andere Dihalomethane vorgeschlagen [76].

\begin{tabular}{|c|c|c|c|}
\hline Normalmode & Energie / $\mathbf{c m}^{-\mathbf{1}}$ & Symmetrie & Zuordnung \\
\hline \hline$\nu_{1}$ & 2983 & $a_{1}$ & $\nu_{s}\left(\mathrm{CH}_{2}\right)$ \\
$\nu_{2}$ & 1361 & $a_{1}$ & $\delta\left(\mathrm{CH}_{2}\right)$ \\
$\nu_{3}$ & 492 & $a_{1}$ & $\nu_{s}\left(\mathrm{CI}_{2}\right)$ \\
$\nu_{4}$ & 122 & $a_{1}$ & $\delta\left(\mathrm{CI}_{2}\right)$ \\
$\nu_{5}$ & 1033 & $a_{2}$ & $\tau \mathrm{CH}_{2}$ \\
$\nu_{6}$ & 3063 & $b_{2}$ & $\nu_{a s}\left(\mathrm{CH}_{2}\right)$ \\
$\nu_{7}$ & 714 & $b_{2}$ & $\delta \mathrm{CH}_{2}$ \\
$\nu_{8}$ & 1152 & $b_{1}$ & $\omega \mathrm{CH}_{2} \mathrm{Kipp}_{1}$ \\
$\nu_{9}$ & 580 & $b_{1}$ & $\nu_{a s}\left(\mathrm{CI}_{2}\right)$ \\
\hline
\end{tabular}

Tabelle 2.1: Normalmoden der Schwingungen in $\mathrm{CH}_{2} \mathrm{I}_{2}$ [58] (die Bezeichnung der Symmetrierassen ist evtl. abweichend zur Symmetrieklassifizierung der elektronischen Zustände in Abb. 2.2 [62].)

\subsection{Einfluss der Lösungsmittelumgebung}

Der folgende Abschnitt beschäftigt sich mit dem Einfluss der Umgebung des Moleküls auf die Dissoziationsdynamik. So verschiebt sich u. a. das Maximum der ersten Absorptionsbande des stationären Spektrums mit zunehmender Lösungsmittelpolarität zu kürzeren Wellenlängen [55].

\subsubsection{Einfluss auf die Molekülschwingungen}

In einigen Arbeiten, insbesondere von Phillips et al. [77,78], wurde mit zeitaufgelöster Resonanz-Raman-Spektroskopie der Einfluss der Solvatation auf die Photodissoziati- 
onsdynamik des Diiodmethans untersucht. Während das Resonanz-Raman-Spektrum in der Gasphase im Wesentlichen nur Fundamentale, einige Obertöne und Kombinationsbanden der drei Franck-Condon-aktiven Moden $\nu_{3}, \nu_{4}$ und $\nu_{9}$ zeigt [61] (siehe Abschnitt 2.1 und Tabelle 2.1), finden sich im Spektrum in Lösung, z. B. Cyclohexan, weitere Obertöne (z. B. $2 \nu_{7}$ ) und Kombinationsbanden (z. B. $\nu_{9}+\nu_{4}, \nu_{9}+2 \nu_{4}$, etc.). Wird für Diiodmethan wie in der Gasphase $C_{2 \mathrm{v}}$-Symmetrie vorausgesetzt, sind für die Kombinationsbanden aus antisymmetrischer C-I-Streckschwingung $\nu_{9}$ und I-C-I-Knickschwingung $\nu_{4}$ keine Resonanzen im Ramanspektrum zu erwarten. Dies liegt daran, dass $\nu_{9}$ eine nicht-totalsymmetrische und $\nu_{4}$ eine totalsymmetrische Mode ist. Daher wird angenommen, dass die Symmetrie des Diiodmethans durch Solvatation reduziert wird und die Kombinationsbanden aus $\nu_{9}$ und $\nu_{4}$ im Resonanz-RamanSpektrum sichtbar werden. Die Größe des Symmetriebruches zeigt sich dabei lösungsmittelabhängig und ist z. B. in Cyclohexan größer als in Methanol. Auf Grund des Symmetriebruches kann es Einflüsse des Lösungsmittels auf die Photodissoziationsdynamik geben. So wird angenommen, dass das Lösungsmittelmolekül z. B. die Bindungsverlängerung einer C-I-Bindung mehr hindert als die der anderen C-I-Bindung. Es wird im Gegensatz zur Gasphase eine C-I-Bindung bevorzugt gebrochen. Ähnliche Untersuchungen zum Lösungsmitteleinfluss auf die Ramanspektren wurden auch an verwandten Halogenmethanen wie $\mathrm{CH}_{2} \mathrm{ClI}[79,80,81,82]$ oder $\mathrm{CF}_{2} \mathrm{I}_{2}$ [83] durchgeführt, die ähnliche Lösungsmitteleinflüsse zeigten.

\subsubsection{Untersuchungen in der Lösungsmittelmatrix}

Eine Reihe von Messungen wurden in Matrix-Umgebungen durchgeführt [32, 33, 84]. Simons und Tatham [84] untersuchten dazu das Absorptionsverhalten einer Diiodmethanprobe nach Anregung mit $254 \mathrm{~nm}$, die in einer Kohlenwasserstoffglasmatrix bei $77 \mathrm{~K}$ eingefroren war. Dabei stellten sie nach längerer UV-Bestrahlung im Absorptionsspektrum zwei neue Banden fest. Eine stärkere Bande bei etwa $380 \mathrm{~nm}$ und eine etwas schwächere Bande bei $530 \mathrm{~nm}$ (siehe Abbildung 2.4). Das Vorhandensein dieser neuen Banden im Absorptionsspektrum nach der Photolyse wurde „gefangenen“ Elektronen zugeordnet. Es wurde davon ausgegangen, dass durch den störenden Einfluss der benachbarten Moleküle das Ionisationspotential stark geändert sein könnte. Danach ist es möglich, die Energien der Rydberg-Orbitale des $\mathrm{CH}_{2} \mathrm{I}_{2}$ durch Störung der Nachbarmoleküle, auf Grund ihrer dichten Packung, zu verändern. Der $n \rightarrow \sigma^{*}$-Übergang kann von Rydberg-Übergängen überlappt werden. Wenn die Nachbarmoleküle ebenfalls Diiodmethan-Moleküle sind, ist es möglich, dass ein angeregtes Rydbergelektron des einen Moleküls durch Tunneln zu einem benachbarten Molekül übertragen werden kann und dort gefangen bleibt. Die beiden $\mathrm{CH}_{2} \mathrm{I}_{2}$-Moleküle bilden dabei einen Ladungstransferkomplex (CT, charge transfer) aus. Weil die Tunnelwahrscheinlichkeit für das Elektron stark abnimmt, wenn die Potentialbarriere steigt, ist dieser Effekt 


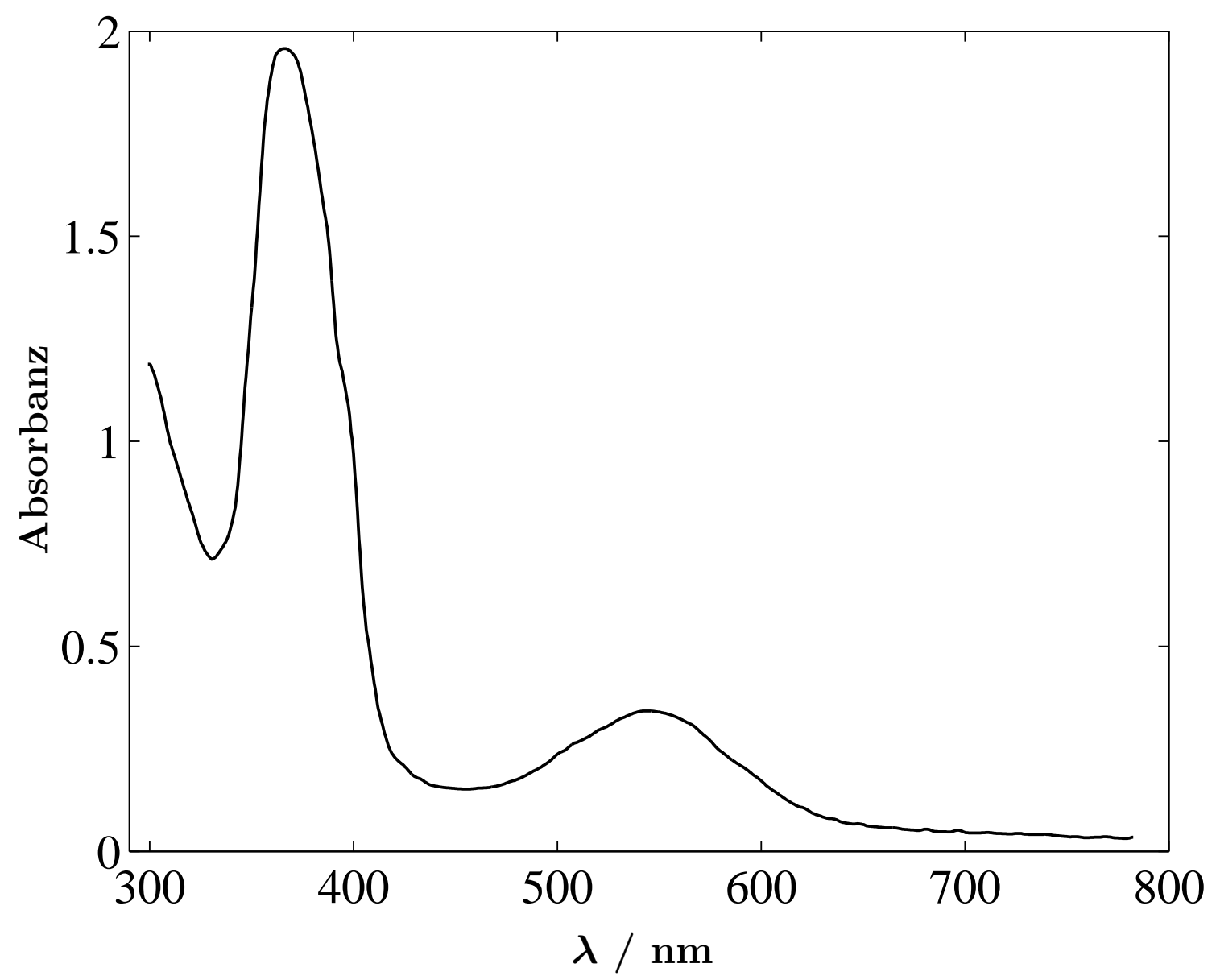

Abbildung 2.4: Absorption der Photoprodukte (Isomer) in einer $\mathrm{N}_{2}$-Matrix nach UVAnregung [33].

nur in der Matrix-Konfiguration beobachtbar. Die gefangenen Elektronen bilden dann die Farbzentren der Absorptionsbanden bei 380 und $530 \mathrm{~nm}$.

Andere Untersuchungen von Diiodmethan nach Photolyse mit $300 \mathrm{~nm}$ in einer 3-Methylpentan (3MP)-Matrix von Mohan et al. [32] lieferten ebenfalls zwei Banden bei 385 und $570 \mathrm{~nm}$ im Absorptionsspektrum. Im Gegensatz zu Simons und Tatham [84] befindet sich das Maximum der zweiten Absorptionsbande weiter im langwelligen Spektralbereich. Die Interpretation des Spektrums ist dabei abweichend zu Simons und Tatham, beinhaltet aber ebenfalls die Bildung eines CT-Komplexes: Nach Aufnahme eines Photons bei $300 \mathrm{~nm}$ wird aus zwei elektronisch angeregten DiiodmethanMolekülen ein CT-Komplex $\left(\mathrm{CH}_{2} \mathrm{I}_{2}{ }^{*}+\mathrm{CH}_{2} \mathrm{I}_{2}{ }^{*} \rightarrow \mathrm{CH}_{2} \mathrm{I}_{2}{ }^{+}+\mathrm{CH}_{2} \mathrm{I}_{2}{ }^{-}\right)$, der seinerseits wieder zerfallen kann und das $\mathrm{CH}_{2} \mathrm{I}$-Radikal bildet. Die Absorptionsbande bei $385 \mathrm{~nm}$ wird dem $\mathrm{CH}_{2} \mathrm{I}_{2}{ }^{+}$, die $570 \mathrm{~nm}$ Bande dem $\mathrm{CH}_{2} \mathrm{I}$ zugeschrieben. 


\subsubsection{Zeitaufgelöste Untersuchungen}

Von Mohan et al. [85] wurden auch transiente Absorptionsmessungen mit $\mu$ s-Zeitauflösung in flüssiger Phase durchgeführt. Dazu wurden transiente Absorptionsspektren und Abklingkurven von Diiodmethan in $\mathrm{N}_{2} \mathrm{O}$-gesättigter, wässriger Lösung und in saurer Lösung durchgeführt. Das zeitliche Verhalten im Absorptionsmaximum bei $380 \mathrm{~nm}$ in $\mathrm{N}_{2} \mathrm{O}$-gesättigter, wässriger Lösung wurde der Dynamik des $\mathrm{CH}_{2} \mathrm{I}_{2} \cdot \mathrm{OH}$-Adduktradikals zugeordnet. Die Signale bei $380 \mathrm{~nm}$ und $570 \mathrm{~nm}$ in saurer Lösung wurden hingegen, abweichend zu den vorangegangenen Arbeiten [32,84], dem $\mathrm{CH}_{2} \mathrm{I}_{2}{ }^{+}$-Intermediat zugeordnet.

Weitere transiente Absorptionsmessungen wurden z. B. von Harris et al. [34] mit fsZeitauflösung durchgeführt. Dabei wurden Pump/Probe-Messungen bei $310 \mathrm{~nm}$ Anregung und $620 \mathrm{~nm}$ Abfrage in flüssiger Lösung, wie z. B. $\mathrm{CCl}_{4}, \mathrm{CHCl}_{3}$ und $\mathrm{CH}_{2} \mathrm{Cl}_{2}$, durchgeführt. Die Signale zeigten einen schnellen Anstieg innerhalb der Zeitauflösung, gefolgt von einem 300-350 fs schnellen Abfall und einem darauf folgenden 10 ps schnellen Anstieg (siehe dazu auch Abbildung 4.3 auf Seite 52). Diese Absorptions-Zeitprofile wurde von Harris et al. dem Absorptionsverhalten des entstehenden $\mathrm{CH}_{2} \mathrm{I}$-Radikals zugeordnet. Das Radikal wird demnach mit hoher Schwingungsanregung (schwingungsheiß) auf einer Zeitskala $<100$ fs aus der Dissoziation von $\mathrm{CH}_{2} \mathrm{I}_{2}$ gebildet. Anschließend kann es nach nur einem Stoß mit dem Lösungsmittelkäfig wieder zu $\mathrm{CH}_{2} \mathrm{I}_{2}$ rekombinieren (Abfall des Signals mit $300 \mathrm{fs}$ ). Der Anstieg mit $\sim 10 \mathrm{ps}$, der von $\mathrm{CCl}_{4}$ über $\mathrm{CHCl}_{3}$ zu $\mathrm{CH}_{2} \mathrm{Cl}_{2}$ schneller wird, rührt demnach von der Schwingungsrelaxation (Abkühlung) des schwingungsheiß gebildeten Radikals her, die auf Grund der Effizienz des Energietransfers lösungsmittelabhängig ist.

Yoshihara et al. [35] führten ebenfalls Pump/Probemessungen mit $268 \mathrm{~nm}$ Anregung und $400 \mathrm{~nm}$ Abfrage am Diiodmethan mit fs-Zeitauflösung in Acetonitril durch. Die erhaltenen Absorptions-Zeitprofile sind denen von Harris et al. sehr ähnlich. Sie bestehen aus einem sehr schnellen Anstieg des Signals ( $200 \mathrm{fs}$ ), einem schnellen $500 \mathrm{fs}$ Abfall und einem Anstieg von 7-13ps (siehe auch Abbildung 4.3 auf Seite 52). Für die beiden schnellen Komponenten wurde keine Abhängigkeit von der Konzentration an $\mathrm{CH}_{2} \mathrm{I}_{2}$ festgestellt, jedoch wird der Anstieg im ps-Bereich mit zunehmender Konzentration schneller. Ferner wurden von ihnen noch transiente Spektren nach $268 \mathrm{~nm}$ Anregung im Probewellenlängenbereich von 300-600 nm mit Nanosekunden-Zeitauflösung durchgeführt. Das transiente Spektrum ist nahezu identisch mit dem MatrixSpektrum von Mohan et al. [32] (siehe Abbildung 2.4). Auf Grund dieses Spektrums und der Konzentrationsabhängigkeit der langsamen Komponente wurden die Femtosekundenmessungen wie folgt interpretiert: Die beiden schnellen Komponenten rühren vom gebildeten $\mathrm{CH}_{2} \mathrm{I}$-Radikal her. Der Anstieg innerhalb der Zeitauflösung stammt demnach von der Absorption des aus der Dissoziation schnell gebildeten Radikals. Der schnelle Abfall wiederum wird der geminalen Rekombination des $\mathrm{CH}_{2} \mathrm{I} / \mathrm{I}$-Radikalpaars 
zum $\mathrm{CH}_{2} \mathrm{I}_{2}$ innerhalb des Lösungsmittelkäfigs zugeordnet [34], während der langsame Anstieg von der Absorption eines $\mathrm{CH}_{2} \mathrm{I}_{2}{ }^{\delta+} \ldots \mathrm{I}^{\delta-}{ }_{-} \mathrm{CT}-\mathrm{Komplexes}$ stammt. Dieser formt schließlich, nach komplettem Ladungsaustausch, $\mathrm{CH}_{2} \mathrm{I}_{2}{ }^{+}$und $\mathrm{I}^{-}[85]$.

\subsubsection{Schwingungsenergietransfer}

Für das Verständnis des inter- und intramolekularen Energietransfers ist Diiodmethan ebenfalls ein oft verwendetes Modellsystem [86, 87, 88, 89, 90]. So wurde etwa mit Femtosekunden-Pump/Probe-Techniken die Schwingungsenergieumverteilung (IVR, intramolecular vibrational energy redistribution) und der Schwingungsenergietransfer (VET, vibrational energy transfer) in das Lösungsmittel in überkritischem $\mathrm{CO}_{2}$ dichteabhängig untersucht [89]. Dabei wurde mit einem IR-Puls bei 1,7 $\mu \mathrm{m}$ ein Oberton oder eine Kombinationsbande der C-H-Streckschwingungen angeregt. Anschließend wurde mit dem Probepuls bei $390 \mathrm{~nm}$ die Absorption in den angeregten Zustand des Diiodmethanes zeitaufgelöst verfolgt. Ein Absorptions-Zeitprofil zeigt zuerst eine ansteigende Komponente, die dem IVR und damit dem Ankommen der Schwingungsenergie in einer Franck-Condon-aktiven C-I-Mode zugeschrieben werden kann. Daran schließt sich eine langsamer abfallende Komponente an, die dem VET, also dem Abfließen der Schwingungsenergie aus einer C-I-Schwingung in das Lösungsmittel, zugeordnet werden kann. Die VET-Zeit fällt mit zunehmender Dichte von 80 ps, bei einer reduzierten Dichte von $\rho / \rho_{c}=1$, bis unter 50 ps bei einer reduzierten Dichte von 1,7. Die IVR-Zeit bleibt in diesem Dichtebereich nahezu konstant $\sim 20 \mathrm{ps}$. Theoretische Ansätze zur Modellierung des VET in fluider Phase, z. B. über das sogenannte IBC (isolated binary collisions)-Modell, welches einen generalisierten Landau-TellerAnsatz verwendet, liefert u. a. Schwarzer et al. [91, 92, 93].

\subsection{Bildung des Isomers}

Die Bildung des $\mathrm{CH}_{2} \mathrm{I}$-I-Isomers nach der Photodissoziation wurde zuerst von Maier et al. [33] mittels Messungen in einer Argon- $/ \mathrm{N}_{2}$-Matrix untersucht. Dabei wurden das Absorptionsverhalten im sichtbaren Spektralbereich und im IR von zahlreichen Dihalomethanen nach UV-Anregung

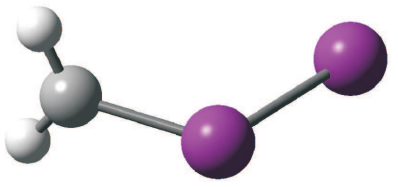

Abbildung 2.5: $\mathrm{CH}_{2} \mathrm{I}-\mathrm{I}$. untersucht. Dabei zeigten einige Dihalomethane eine intensive Färbung bei 15 bzw. $77 \mathrm{~K}$ in der Matrix, welche bei Erhitzung wieder verschwand. Das Absorptionsspektrum für das Diiodmethan wies eine Bande bei $370 \mathrm{~nm}$, eine schwächere Bande bei 545 nm (siehe Abbildung 2.4) im sichtbaren Spektralbereich und neue Banden im IR auf. Diese Banden verschwanden nach erneuter Bestrahlung 
in das Absorptionsmaximum des Photoproduktes bei $370 \mathrm{~nm}$ und das Absorptionsspektrum im sichtbaren Spektralbereich und im IR des $\mathrm{CH}_{2} \mathrm{I}_{2}$ stellte sich quantitativ wieder ein. Dieses führte zu der Vermutung, dass es sich um ein Isomer handelt, dessen Bildungsreaktion in der Matrix auch umkehrbar ist. Für einige andere Dihalomethane (z. B. Dichlormethan, Dibrommethan) wurden die IR-Spektren ihrer Isomere mit Frequenzen aus quantenchemischen Rechnungen auf SCF (self consistent field) oder störungstheoretischem (MP2) Niveau verglichen und eine gute Übereinstimmung festgestellt. Für diese Isomere wurden Energieminima bei nichtplanaren Strukturen mit

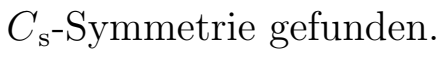

Glukhovtsev und Bach berechneten für das $\mathrm{CH}_{2} \mathrm{I}$-I-Isomer mit quantenchemischen Methoden eine spiegelsymmetrische Gleichgewichtsgeometrie [94]. Es wurde eine mögliche Geometrie eines Übergangszustandes für die Isomerisierungsreaktion von $\mathrm{CH}_{2} \mathrm{I}_{2}$ $\mathrm{zu} \mathrm{CH}_{2} \mathrm{I}-\mathrm{I}$ berechnet, die ebenfalls $C_{\mathrm{s}}$-Symmetrie aufweist. Die Strukturdaten der Gleichwichtsgeometrie wurden u. a. mit Dichtefunktionalmethoden (DFT, density functional theory) berechnet (siehe auch Tabelle 2.2). Außerdem wurden mit der G2Methode thermodynamische Größen, wie die Standardbildungsenthalpie und die Dissoziationsenergie des Isomers, quantenchemisch ermittelt.

Darauf aufbauend untersuchten Orel und Kühn [95] die 2-dimensionale, kartesische Struktur der Reaktionspotentialfläche mit quantenchemischen Methoden. Während die Gleichgewichtsstruktur und die Normalmoden für das $\mathrm{CH}_{2} \mathrm{I}$-I-Isomer in sehr guter Übereinstimmung mit den Daten von Glukhovtsev und Bach [94] liegt, weicht die Struktur des Übergangszustandes stark ab. Die Struktur zeigt sich, auf Grund ihres sehr flachen Minimums, dabei besonders abhängig vom verwendeten Basissatz. Die Rechnungen ergaben einen sehr flachen Verlauf der Reaktionspotentialfläche, so dass es nicht möglich war den kompletten Verlauf bis zur Gleichgewichtsgeometrie des Isomers zu verfolgen. Jedoch konnten die Energiedifferenz der beiden Isomere zu 3,1 eV und die energetische Höhe des Übergangszustandes für die $\mathrm{CH}_{2} \mathrm{I}_{2} \quad \mathrm{zu} \mathrm{CH}_{2} \mathrm{I}$-I-Isomerisierung zu 3,7 eV ermittelt werden.

Zeitaufgelöste Messungen zur Bildung des Isomers in flüssiger Phase stammen von Åkesson et al. [36]. Sie untersuchten mit fs-Pump/Probe-Techniken die Dissoziationsdynamik von Diiodmethan in Acetonitril. Dabei wurde die Probe mit $310 \mathrm{~nm}$ angeregt und Absorptions-Zeitprofile im Bereich von 290-1200 nm detektiert. Ferner wurden im Spektralbereich von 290-780 nm zeitaufgelöste Spektren aufgenommen. Zu frühen Zeiten (bis $300 \mathrm{fs}$ ) nach Anregung finden sich zwei auffällige Banden im transienten Spektrum bei $\sim 350 \mathrm{~nm}$ und bei Wellenlängen größer als $650 \mathrm{~nm}$. Im mittleren Zeitbereich, zwischen 0,5 und 10 ps verschmälert sich die Bande bei $350 \mathrm{~nm}$ und gewinnt an Intensität. Die Bande im langwelligeren Bereich $(>650 \mathrm{~nm})$ verschiebt sich zu kürzeren Wellenlängen. Nach langen Zeiten $\geq 50$ ps hat das transiente Spektrum die gleiche Gestalt wie das Absorptionsspektrum in der Matrix [33] bzw. das Nanosekundenabsorptionsspektrum [35] (siehe Abbildung 2.4). Auf Grund der Tatsache, dass das 
Langzeitspektrum mit dem Matrix-Spektrum identisch ist, gehen Åkesson et al. davon aus, dass es sich um die Bildung des Isomers handeln muss. Das Spektrum zu frühen Zeiten entspricht demnach dem schwingungsheiß gebildeten $\mathrm{CH}_{2} \mathrm{I}$-I-Isomer, welches über Schwingungsrelaxation (IVR und VET) auf einer Zeitskala von 5-50 ps abkühlt. Absorptions-Zeitprofile im langwelligen Bereich von 610-1200 nm zeigen nach dem ersten schnellen Anstieg des Signals einen schnellen Abfall (350-450 fs), gefolgt von einem langsamen Anstieg (1-3 ps) und einem langsamen Abfall (3,5-6 ps) des Signals. Ähnliches Verhalten wird im sehr kurzwelligen Bereich zwischen 290 und $320 \mathrm{~nm}$ gefunden. In Richtung der Absorptionsmaxima nimmt die langsam abfallende Komponente immer weiter ab und verschwindet dann ganz. Die Zeiten des schnellen Abfalls und des Anstiegs werden dabei größer. Im Bereich der beiden Maxima sind die Profile äquivalent zu denen von Yoshihara et al. [35] und Harris et al. [34] (siehe Abschnitt 2.2.3 und Abbildung 4.3 auf Seite 52), jedoch beinhaltet die Interpretation in diesem Falle die Bildung des $\mathrm{CH}_{2} \mathrm{I}$-I-Isomers.

Der schnelle Anstieg des Signals innerhalb der Zeitauflösung des Experiments wird der transienten Absorption des angeregten $\mathrm{CH}_{2} \mathrm{I}_{2}$ zugeordnet. Da er sowohl in einem großen Bereich um $350 \mathrm{~nm}$ als auch um $700 \mathrm{~nm}$ präsent ist, wird die Absorption des $\mathrm{CH}_{2}$ I-Radikals als Hauptursache ausgeschlossen. Jedoch tragen im kürzerwelligen Spektralbereich das Ausbleichen des $\mathrm{CH}_{2} \mathrm{I}_{2}$-Signals, die Radikalabsorption und die Isomerabsorption zu frühen Zeiten etwas zum Gesamtsignal bei. Der schnelle Abfall mit $\sim 350$ fs ist demnach mit dem Herauslaufen des, im angeregten Zustand erzeugten, Wellenpaketes aus der Franck-Condon-Region zu erklären. Im Zeitbereich von 350 fs bis 1 ps bilden sich dann innerhalb des Lösungsmittelkäfigs das $\mathrm{CH}_{2} \mathrm{I} / \mathrm{I}$-Radikalpaar und das schwingungsheiße $\mathrm{CH}_{2} \mathrm{I}-\mathrm{I}$-Isomer. Die Schwingungsrelaxation des Isomers findet auf einer 10 ps Zeitskala statt.

Unter ähnlichen Bedingungen, d.h. Diiodmethan in Acetonitril, wurden von Zheng und Phillips [96] transiente Resonanz-Raman-Messungen an den Photoprodukten der Dissoziation von $\mathrm{CH}_{2} \mathrm{I}_{2}$ durchgeführt. Während das Resonanz-Raman-Spektrum in der Gasphase des $\mathrm{CH}_{2} \mathrm{I}_{2}$ aus Fundamentalen, Obertönen und Kombinationsbanden dreier Franck-Condon-aktiver Moden $\nu_{3}, \nu_{4}$ und $\nu_{9}$ bei 701, 619 und $128 \mathrm{~cm}^{-1}$ besteht, finden sich im transienten Resonanz-Raman-Spektrum Fundamentale, Obertöne und Kombinationsbanden von vier Franck-Condon-aktiven Moden bei 640, 496, 128 und $\sim 110 \mathrm{~cm}^{-1}$. Diese Frequenzen wurden mit quantenchemisch berechneten Frequenzen aller bisher in der Literatur diskutierten Spezies, $\mathrm{CH}_{2} \mathrm{I}, \mathrm{CH}_{2} \mathrm{I}_{2}{ }^{+}$und $\mathrm{CH}_{2} \mathrm{I}-\mathrm{I}$, verglichen. Als Ursprung dieses Photoproduktspektrums kommt demnach nur das $\mathrm{CH}_{2} \mathrm{I}-\mathrm{I}-$ Isomer in Frage. Dieses bestätigt also die Vermutung von Åkesson etal. [36], dass es sich bei dem Photoprodukt um das $\mathrm{CH}_{2} \mathrm{I}-\mathrm{I}$-Isomer handelt. Intensive elektronische Übergänge wurden in Berechnungen von Zheng und Phillips um $400 \mathrm{~nm}$ in relativ guter Übereinstimmung mit der intensiven Bande bei 370 nm gefunden [96]. Bei der Bande im Bereich von $570 \mathrm{~nm}$ könnte es sich demnach eventuell um einen Triplett-Übergang des $\mathrm{CH}_{2} \mathrm{I}-\mathrm{I}$-Isomers handeln. 
In weiteren transienten Resonanz-Raman-Messungen mit ps-Zeitauflösung in polaren (Acetonitril) und unpolaren (Cyclohexan) Lösungsmitteln von Phillips et al. [37] erschienen die dem Isomer zugeordneten Banden innerhalb von 5-8 ps nach der Anregung. Diese Banden wachsen mit der Zeit an und verschieben sich leicht zu höheren Frequenzen. Dieses Verhalten deutet auf ein Abkühlen des Photoproduktes hin. Phillips et al. schließen daraus, dass das schwingungsheiße $\mathrm{CH}_{2} \mathrm{I}-\mathrm{I}$-Isomer innerhalb von wenigen Pikosekunden gebildet wird und anschließend auf einer Zeitskala von mehreren 10 ps abkühlt.

Von Phillips etal. wurden auch weitere Arbeiten veröffentlicht, die sich mit quantenchemischen Rechnungen und Resonanz-Raman-Messungen am $\mathrm{CH}_{2} \mathrm{I}-\mathrm{I}$ beschäftigen $[97,98]$. Diese diskutieren u. a. die Bildung eines $\mathrm{CH}_{2} \mathrm{I}_{2} \cdots$ I-Komplexes als weiteres sekundäres Photoprodukt aus einer bimolekularen Reaktion von $\mathrm{CH}_{2} \mathrm{I}_{2}$ mit IodAtomen.

Weitere theoretische und ramanspektroskopische Arbeiten aus jüngster Zeit befassen sich mit Reaktionen des photochemisch gebildeten $\mathrm{CH}_{2} \mathrm{I}$-I-Isomers mit Olefinen, wie z. B. mit Zink in der Simmons-Smith [52]-Cyclopropanierungsreaktion $[99,100,101,102,103]$. Dabei wird dem $\mathrm{CH}_{2} \mathrm{I}-\mathrm{I}$-Isomer eine wesentliche Rolle im Photocyclopropanierungsmechanismus zugeschrieben.

\begin{tabular}{|c|c|c|c|c|c|}
\hline \multicolumn{4}{|c|}{ Schwingungen } & \multicolumn{2}{|c|}{ Geometrie } \\
\hline Mode & $E\left[\mathrm{~cm}^{-1}\right]$ & Symmetrie & Zuordnung & Parameter & \\
\hline$\nu_{1}$ & 3131 & $a^{\prime}$ & $\nu_{s}\left(\mathrm{CH}_{2}\right)$ & $\mathrm{R}(\mathrm{C}-\mathrm{I})$ & $1,957 \AA$ \\
\hline$\nu_{2}$ & 1340 & $a^{\prime}$ & $\delta\left(\mathrm{CH}_{2}\right)$ & $\mathrm{R}(\mathrm{I}-\mathrm{I})$ & $3,042 \AA$ \\
\hline$\nu_{3}$ & 755 & $a^{\prime}$ & $\nu\left(\mathrm{CI}_{2}\right)$ & $\mathrm{R}(\mathrm{C}-\mathrm{H})$ & $1,091 \AA$ \\
\hline$\nu_{4}$ & 619 & $a^{\prime}$ & $\omega\left(\mathrm{CH}_{2}\right)$ & $\angle(\mathrm{C}-\mathrm{I}-\mathrm{I})$ & $118,2^{\circ}$ \\
\hline$\nu_{5}$ & 128 & $a^{\prime}$ & $\nu(\mathrm{I}-\mathrm{I})$ & $\angle(\mathrm{H}-\mathrm{C}-\mathrm{I})$ & $119,1^{\circ}$ \\
\hline$\nu_{6}$ & 99 & $a^{\prime}$ & $\delta(\mathrm{C}-\mathrm{I}-\mathrm{I})$ & $\angle(\mathrm{H}-\mathrm{C}-\mathrm{I}-\mathrm{I})$ & $90,0^{\circ}$ \\
\hline$\nu_{7}$ & 3281 & $a^{\prime \prime}$ & $\nu_{a s}\left(\mathrm{CH}_{2}\right)$ & & \\
\hline$\nu_{8}$ & 865 & $a^{\prime \prime}$ & $\delta\left(\mathrm{CH}_{2}\right)$ & & \\
\hline$\nu_{9}$ & 447 & $a^{\prime \prime}$ & $\tau\left(\mathrm{CH}_{2}\right)$ & & \\
\hline
\end{tabular}

Tabelle 2.2: Quantenchemisch berechnete Normalmoden der Schwingungen (B3LYP/ Sadlej-PVTZ) [97] und Gleichgewichtsgeometrie (B3LYP/Sadlej-PVTZ) [96] von $\mathrm{CH}_{2} \mathrm{I}-\mathrm{I}$. 



\section{Kapitel 3}

\section{Experimentelle Technik}

Zur Untersuchung der Kinetik und Dynamik der Isomerisierung des Diiodmethans ist eine Methode mit hoher Zeitauflösung nötig. Die in dieser Arbeit verwendete Technik wird im Folgenden beschrieben.

\subsection{Wahl der Methode}

Zur Untersuchung der Isomerisierungsreaktion des Diiodmethans wird die Pump/Probe-Spektrometrie (engl.: pump-probe, auch Testimpuls-Spektrometrie genannt) verwendet [104]. Dabei wird ein intensiver Laserpuls (Pumppuls) in eine Probe eingestrahlt, die somit angeregt wird. Anschließend wird mit einem schwachen Abfragepuls (Probepuls) nach einer definierten einstellbaren Zeitspanne das Vermögen der Probe zur Absorption, Verstärkung, Reflexion oder Polarisationsdrehung des Testimpulses gemessen. Dabei können die Wellenlängen des Pump- und des Probepulses unterschiedlich voneinander sein, um z. B. die transiente Absorption aus einem elektronisch angeregten Zustand in einen noch höher liegenden elektronischen Zustand zu detektieren (transiente Absorption). Als Messgröße dient dabei meist die Änderung der optischen Dichte $\triangle O D$ zwischen einer Messung der Absorption mit und ohne Anregung der Probe.

Zum Betrieb eines solchen Pump-Probe-Spektrometers mit ultrakurzer Zeitauflösung werden hauptsächlich Titan:Saphir-Lasersysteme verwendet, die mittlerweile in einer Vielzahl von Ausführungen kommerziell zur Verfügung stehen. Eine Übersicht über die Kurzpulserzeugung mit Titan:Saphir-Lasern geben unter anderem [105, 106, 107]. Das in dieser Arbeit verwendete Lasersystem wird im Folgenden beschrieben. 


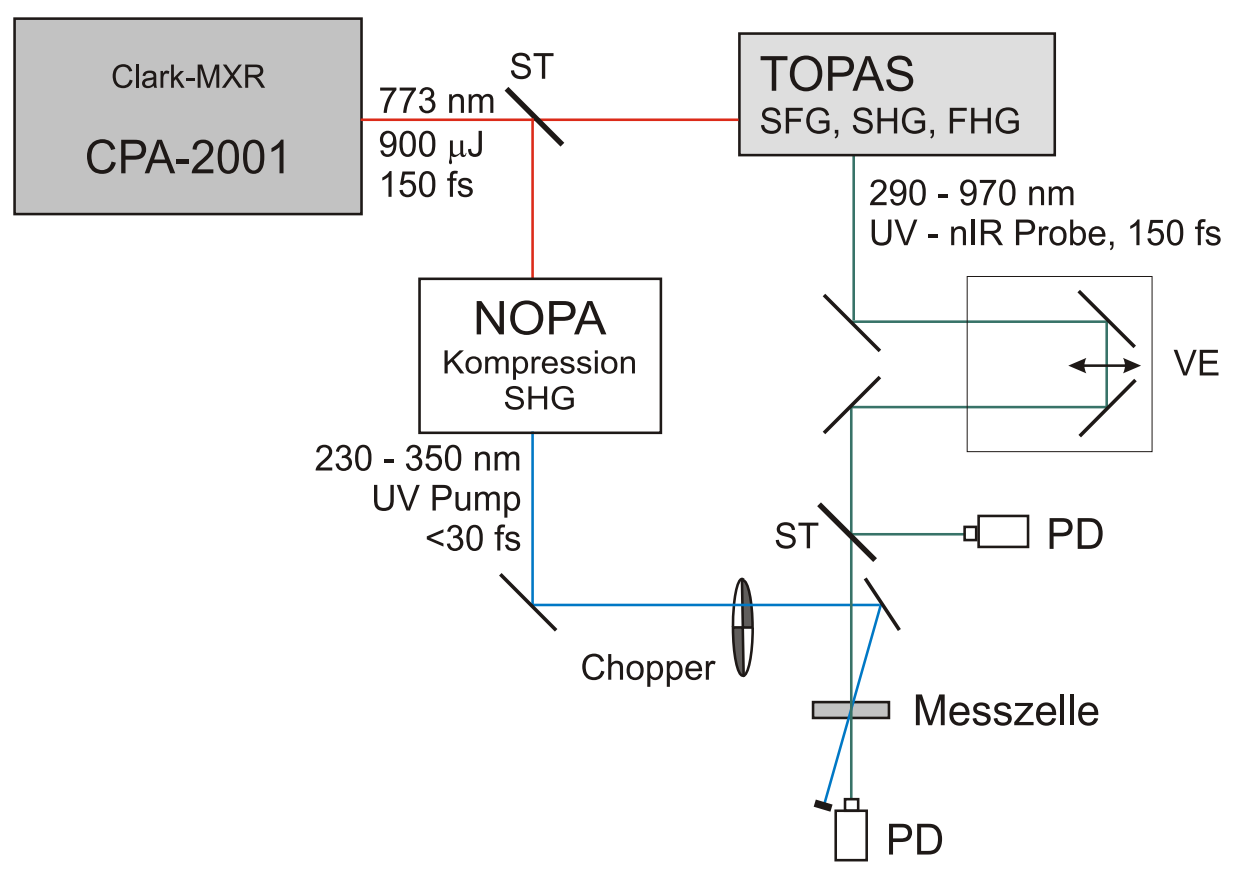

Abbildung 3.1: Das verwendete Lasersystem im Überblick.

\subsection{Das Lasersystem im Überblick}

Der schematische Aufbau des Lasersystems (siehe auch [108,109,110]) und der Detektion ist in Abbildung $3.1 \mathrm{zu}$ finden. Es besteht aus einem kommerziellen regenerativ verstärkten [111] Titan:Saphir-Laser (Clark MXR Inc. CPA-2001 [112]) der Femtosekundenpulse mit einer Dauer von etwa 150 fs bei einer Zentralwellenlänge von $773 \mathrm{~nm}$ und einer Impulsfolge von etwa $1 \mathrm{kHz}$ liefert. Die mittlere Energie pro Puls beträgt dabei $880 \mu \mathrm{J}$. Der Ausgangsstrahl des CPA-2001 wird mittels eines dielektrischen Strahlteilers (ST) in einen reflektierten Anteil (33\%) und einen transmittierten Anteil (67\%) aufgespalten.

Der reflektierte Anteil wird zum Pumpen eines kommerziellen (Clark MXR Inc.) zweistufigen NOPA (Noncollinear Optical Parametric A mplifier [113]) nach dem Prinzip von Riedle $[114,115]$ verwendet. Dieses System liefert Laserpulse im sichtbaren Spektralbereich von 460-700 nm mit einer Pulslänge nach Kompression von $<30$ fs und einer mittleren Pulsenergie von bis zu $7 \mu \mathrm{J}$. Nach Frequenzverdoppelung sind (Pump-)Pulse von 230-350 nm verfügbar.

Der andere Teil des Laserlichtes des CPA-2001 pumpt einen TOPAS ( Travelling-wave Optical Parametric Amplifier of $\boldsymbol{S}$ uperfluorescence [116]) der Firma Light Conversion. Dieser erlaubt, mit optionaler Nachschaltung von verschiedenen nichtlinearen 


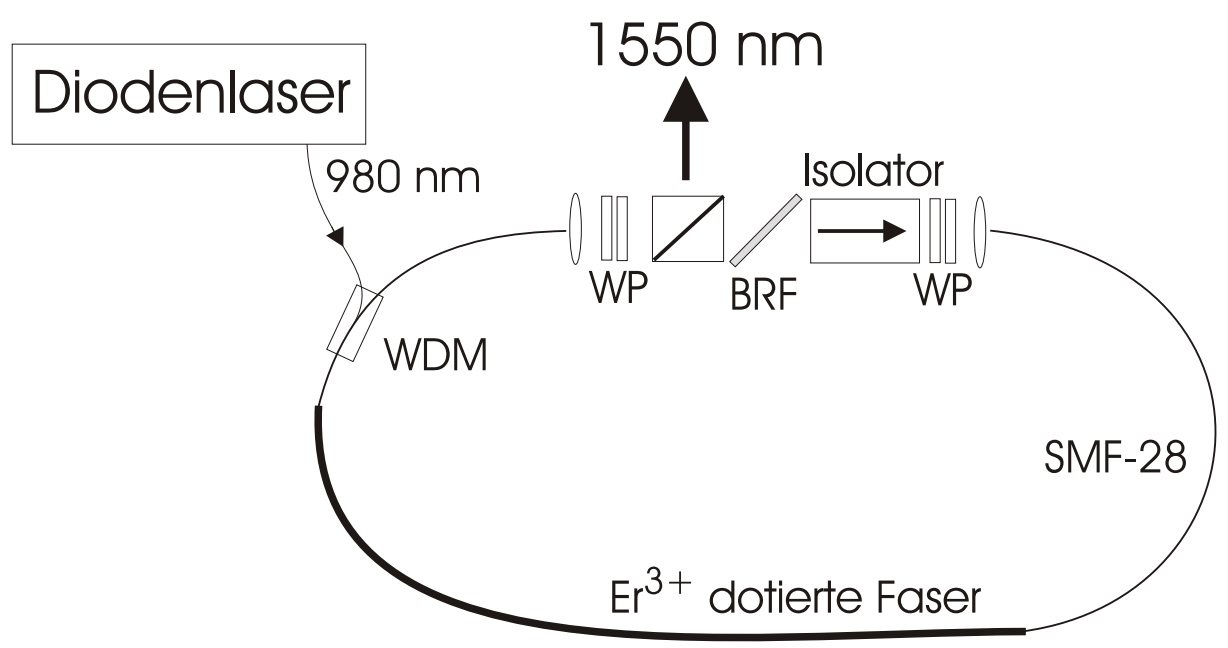

Abbildung 3.2: Prinzip des Faserlasers $[112,118]$.

optischen Prozessen (SHG, SFG bzw. FHG [117]), die Erzeugung von (Probe)-Pulsen im Wellenlängenbereich von 190-2750 nm.

Der Messaufbau besteht im Wesentlichen aus der Messzelle (siehe Abschnitt 3.5), in der Pump- und Probepuls unter einem kleinen Winkel räumlich überlagert werden. Die zeitliche Differenz zwischen Anregung und Abfrage wird über eine computergesteuerte Verschiebebühne (VE) (Physik Instrumente, PI M-415.DG) erreicht. Die Detektion geschieht über zwei intergrierende Photodioden (PD).

Eine detaillierte Beschreibung der einzelnen Komponenten des Messaufbaus findet sich in den folgenden Abschnitten.

\subsection{Die Komponenten des Lasersystems}

\subsubsection{Der CPA-2001}

Bei dem CPA-2001 handelt es sich um einen regenerativ verstärkten Titan:SaphirLaser [111]. Er ist in zwei übereinander liegende Sektionen eingeteilt. In der unteren Sektion befindet sich ein Diodenlaser als optische Pumpe für einen modengekoppelten Faserlaser [118] sowie ein Pulsstrecker. In der oberen Sektion befindet sich der eigentliche, Ti:Saphir-basierte, regenerative Verstärker, der von einem Nd:YAG-Laser optisch gepumpt wird sowie der Pulskompressor.

Das Diodenlaserlicht der Wellenlänge $980 \mathrm{~nm}$ wird über einen 980/1550 nm WDMKoppler (wavelength division multiplexing) in einen Ringresonator eingekoppelt und 
pumpt optisch das laseraktive Medium [119], eine $\mathrm{Er}^{3+}$-dotierte Faser (siehe Abbildung 3.2). Die Faser emittiert bei $1550 \mathrm{~nm}$ und weist normale Dispersion auf. Um die Gruppengeschwindigkeitsdispersion (group velocity dispersion, GVD) der dotierten Faser und der anderen optischen Elemente des Laserresonators zu kompensieren, ist ein Stück Standardglasfaser (single mode fiber, SMF-28) mit anormaler Dispersion in den Resonator eingebracht. Der gepulste Betrieb des Ringlasers wird über das polarisationsadditive Modenkoppeln (polarisation additive pulse modelocking, APM [118]) realisiert, welches über die korrekte Einstellung der vier Verzögerungsplatten (WP) erreicht werden kann. Der stabile Modenkopplungsbetrieb geht dabei auf Kosten der Ausgangsenergie des Lasers. Die Pulsfrequenz des Faserlasers beträgt 27,5 MHz. Als weitere Elemente dienen noch ein doppelbrechender Filter (birefringten filter, BRF) als Bandpass und ein optischer Isolator zum Betrieb des Oszillators in nur einer Resonatorrichtung. Zur Justage und zur Überwachung des selbsteinsetzenden Pulsbetriebes müssen nur gelegentlich die Verzögerungsplättchen nach dem Isolator leicht verstellt werden. Der ausgekoppelte Puls kann mittels einer Gitterkompression [120] auf etwa $100 \mathrm{fs}$ verkürzt werden. Anschließend werden die um $1550 \mathrm{~nm}$ zentrierten Pulse in einem Lithiumtriborat-Kristall (LBO) frequenzverdoppelt. Die so entstandenen Pulse mit einer Zentralwellenlänge von etwa $775 \mathrm{~nm}$ werden in einem Strecker auf eine Pulslänge von einigen Pikosekunden zeitlich verbreitert um in der nachfolgenden regenerativen Verstärkung eine Beschädigung der Optiken durch zu hohe Spitzenintensitäten zu vermeiden. Die so erhaltenen Seedpulse werden in den eigentlichen Verstärker eingekoppelt.

Zentrales Element des Verstärkers ist ein Ti:Saphir-Kristall, in dem eine Besetzungsinversion durch optisches Pumpen mit einem frequenzverdoppelten Nd:YAG-Laser (ORC-1000, Clark MXR Inc.) bei $532 \mathrm{~nm}$ erreicht wird. Der Nd:YAG-Laser seinerseits wird durch eine Krypton-UV-Lampe gepumpt, mit einem akustooptischen Modulator mit einer Frequenz von $1 \mathrm{kHz}$ kreisgütegeschaltet (Q-Switch [121]) und innerhalb der Kavität mit einem KTP-Kristall $\left(\mathrm{KTiOPO}_{4}\right)$ frequenzverdoppelt. Zeitverzögert zu der mit $1 \mathrm{kHz}$ erzeugten Besetzungsinversion werden die Seedpulse in die Verstärkerkavität mittels einer Pockelszelle eingekoppelt. Im Titan:Saphir-Kristall kann der Seedpuls nun die Besetzungsinversion durch stimulierte Emission „abräumen“ und dabei verstärkt werden. Dieses kann nun viele Male beim erneuten Durchlaufen des Pulszuges durch die Verstärkerkavität erfolgen bis die Verstärkung ihr Maximum erreicht hat. Dann koppelt die Pockelszelle den Puls wieder aus dem Resonator aus. Der genaue Zeitpunkt der Ein- und Auskoppelung wird durch einen HochfrequenzHochspannungstreiber (DT505, Clark MXR Inc.) eingestellt, und es wird ein wie in Abbildung 3.3 gezeigtes Verstärkerprofil erhalten. Der Abstand zwischen zwei Pulsen entspricht gerade einem Resonatorumlauf. Dieses Verstärkerprofil wird stets mit einem digitalen Oszilloskop (LeCroy 9362) überwacht.

Nach der Auskopplung aus dem Resonator des Verstärkers durchläuft der Puls eine Gitterkompression und wird auf eine Pulsdauer von ca. 150 fs komprimiert. Eine Au- 


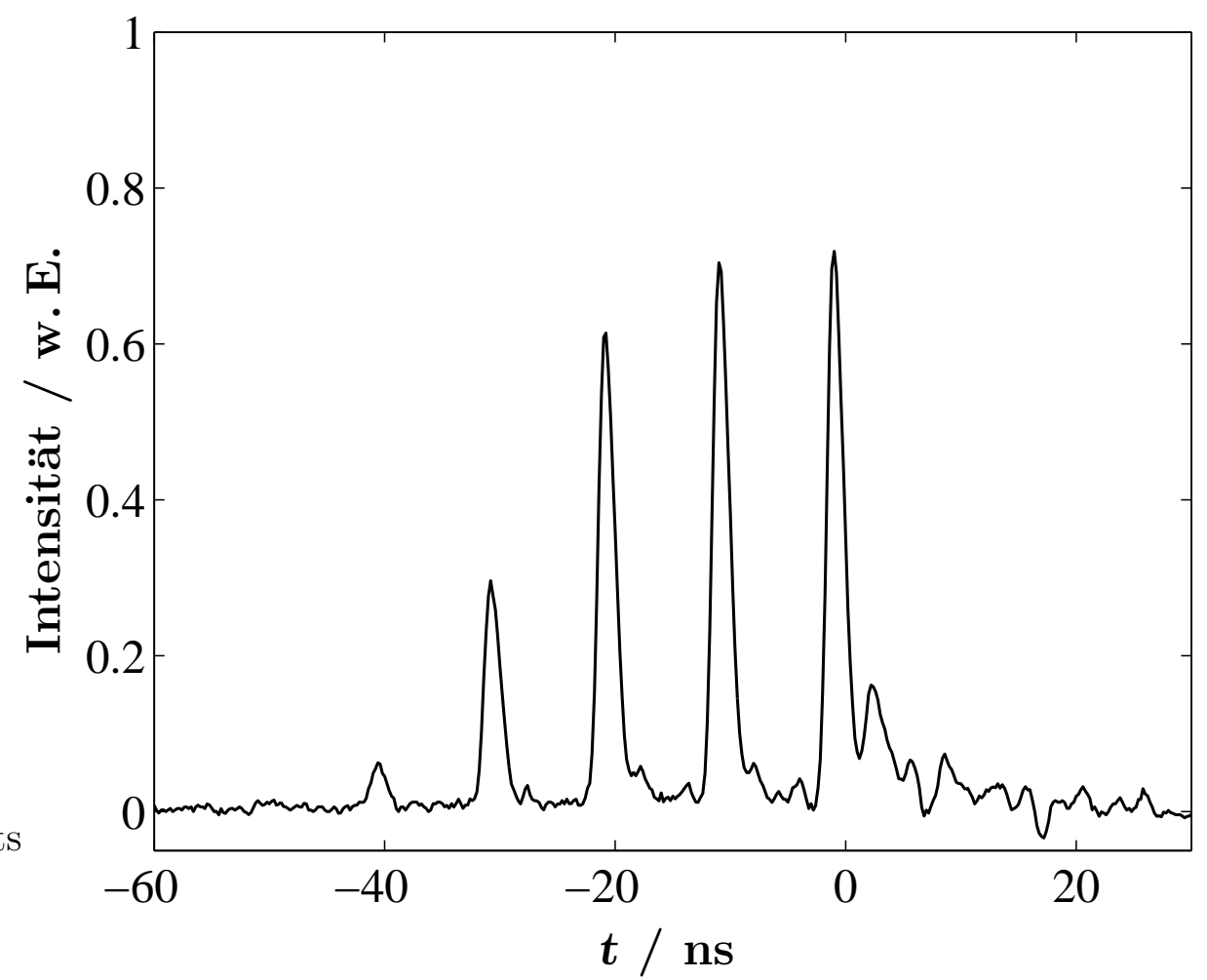

Abbildung 3.3: Verstärkungsprofil des Seedpulses im Resonator des Ti:SaphirVerstärkers.

tokorrelationsmessung nach dem Prinzip des Michelson-Interferometers lieferte unter der Annahme eines lorenzförmigen Zeitprofils eine Pulslänge von 120 fs (siehe Abbildung 3.4).

In der Abbildung der Autokorrelation lassen sich deutliche Schultern erkennen, die von einem schwachen Pikosekundenuntergrund herrühren, der durch höhere Beugungsordnungen des Gitterkompressors entsteht. Dieser Untergrund verschwindet in den folgenden nichtlinearen optischen Prozessen und hat für die Zeitauflösung des Experimentes keine Bedeutung mehr.

Der CPA-2001-Laser liefert p-polarisierte Pulse mit einer Repetitionsrate von $1 \mathrm{kHz}$ bei einer Zentralwellenlänge von $773 \mathrm{~nm}$ und einer mittleren Pulsenergie von $880 \mu \mathrm{J}$ bei einer Pulslänge von etwa 150 fs.

\subsubsection{Erzeugung des Pumppulses im NOPA}

Die für das Experiment benötigten Pumppulse werden in einem nichtkollinear gepumpten optisch parametrischen Verstärker (NOPA) mit anschließender Pulskompression 


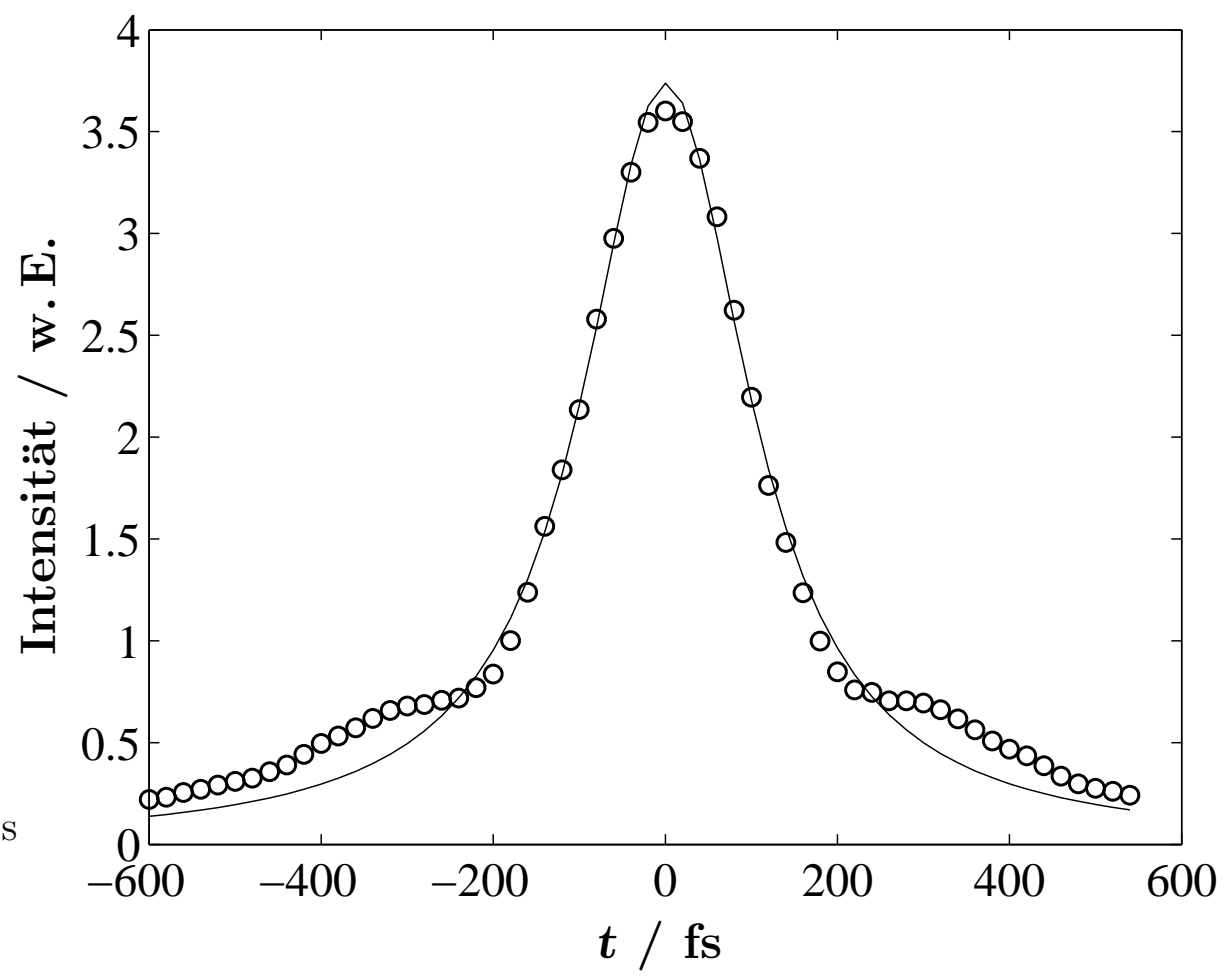

Abbildung 3.4: Autokorrelationsmessung der Pulse des CPA-2001. Die Halbwertsbreite einer lorenzförmigen Anpassung beträgt 120 fs.

und Frequenzverdopplung erzeugt. Der Strahlengang und der prinzipielle Aufbau des Gerätes sind in Abbildung 3.5 dargestellt. Der NOPA wird mit 33\% der Laserfundamentalen bei $773 \mathrm{~nm}$ betrieben.

Das grundlegende Prinzip des optisch parametrischen Verstärkers beruht auf der Theorie des 3-Wellen-Mischens [117]. Wird eine starke Pump- $\left(\omega_{\text {Pump }}\right)$ und eine Signalwelle $\omega_{\text {Signal }}\left(\right.$ mit $\left.\omega_{\text {Pump }}>\omega_{\text {Signal }}\right)$ in ein nichtlineares optisches Medium eingestrahlt, so wird $\omega_{\text {Signal }}$ verstärkt und eine weitere Frequenz $\omega_{\text {Idler }}$, der so genannte Idler, auf Kosten der Energie des Pumpulses, erzeugt. Für die 3 Wellen müssen sowohl Energieerhaltung als auch die Phasenanpassungsbedingung erfüllt sein:

$$
\begin{aligned}
\omega_{\text {Idler }} & =\omega_{\text {Pump }}-\omega_{\text {Signal }} \\
\Delta k & =k_{\text {Idler }}+k_{\text {Signal }}-k_{\text {Idler }} \stackrel{!}{=} 0 .
\end{aligned}
$$

Bei Verwendung eines doppelbrechenden Kristalls wie z. B. BBO (Beta-Barium-Borat, $\left.\beta-\mathrm{BaB}_{2} \mathrm{O}_{4}\right)$ als nichtlineares optisches Medium und kollineare Überlagerung des Signalund Pumpstrahls in einem 1-2 mm langen Kristall werden typischerweise Pulse von einer Länge von nicht kleiner als 100 fs erhalten. Dies liegt daran, dass es nicht möglich ist, die Gruppengeschwindigkeiten aller 3 Wellen zu kontrollieren und eine Phasenan- 


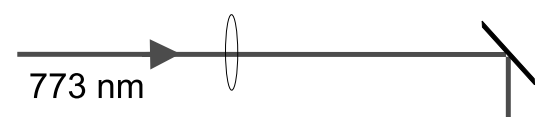

\section{Stufe}

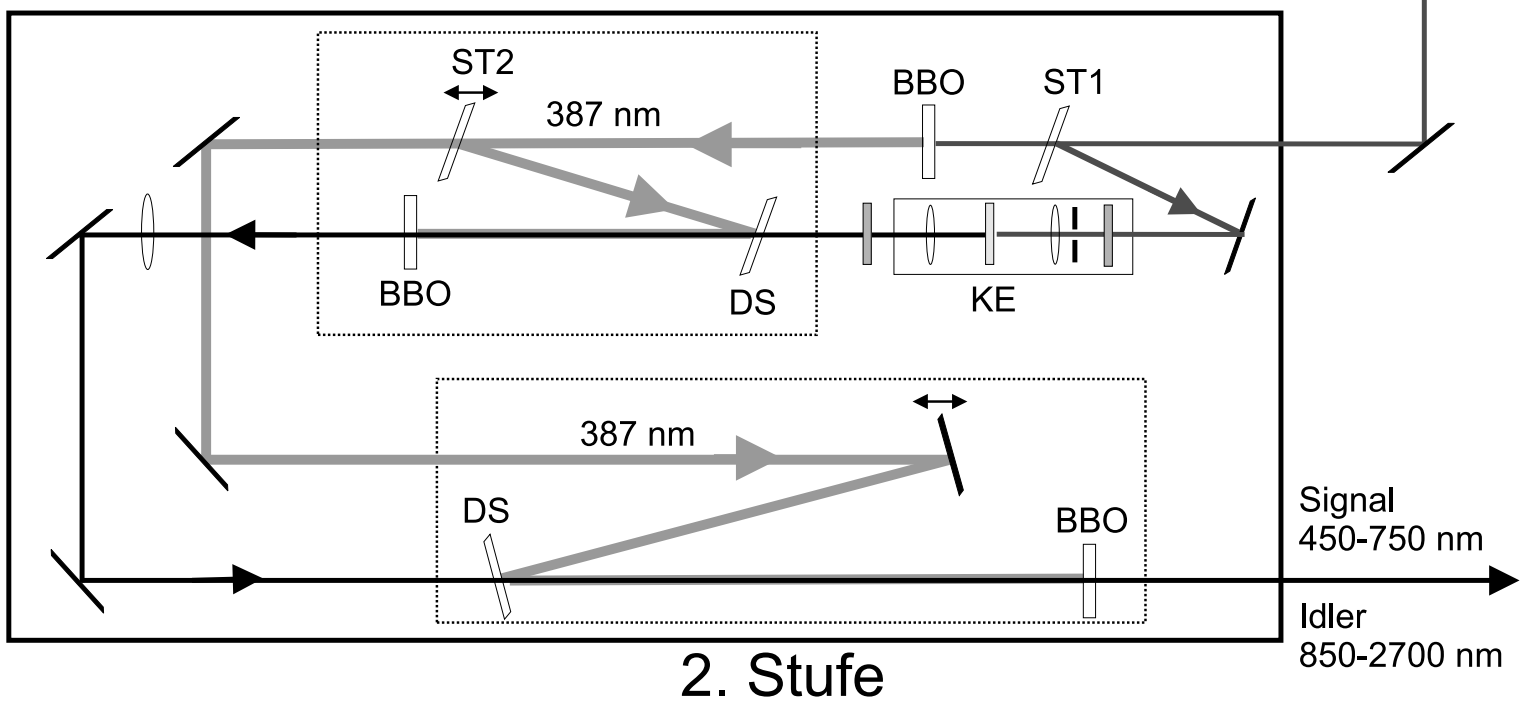

Abbildung 3.5: Schematischer Aufbau des verwendeten NOPA [113].

passung über die gesamte Kristalllänge aufrecht zu erhalten. Deshalb arbeitet der parametrische Verstärker nach dem Riedle-Prinzip [114,115] mit einer nichtkollinearen Anordnung von Signal- und Pumpwelle. Bei einem bestimmten Winkel $\alpha$ zwischen beiden Wellen läuft die Projektion in Signalrichtung des erzeugten Idler mit der gleichen Gruppengeschwindigkeit $v_{\mathrm{g}}$ wie der Signalpuls durch den Kristall:

$$
v_{\mathrm{g}, \text { Signal }}=\cos (\alpha) \cdot v_{\mathrm{g}, \mathrm{Idler}} .
$$

Diese Anordnung ermöglicht eine bessere Effizienz der Verstärkung und verhindert eine zeitliche Verbreiterung der Pulse. Im NOPA wird als Signalpuls ein in einem Saphir-Kristall erzeugtes Weißlichtkontinuum [122] verwendet. Dieses entsteht nach Einstrahlen der Laserfundamentalen von $773 \mathrm{~nm}$ durch Ramanübergänge im Saphir. Das Spektrum des Weißlichtkontinuums ist im Wellenlängenbereich von 460-700 nm flach und nahezu strukturlos.

Der für den NOPA vorgesehene Teil der Fundamentalen erreicht über zwei dielektrische, hochreflektierende Spiegel $\left(\mathrm{HR} 775,45^{\circ}\right)$ den Eingang des NOPA. Durch den dielektrischen Strahlteiler (ST1) werden 2\% reflektiert und zur Kontinuumserzeugung (KE) im Saphirkristall verwendet. Der übrige Anteil wird in einem BBO-Kristall $\left(8 \times 8 \times 0,7 \mathrm{~mm}^{3}, \Theta=30^{\circ}\right.$, Typ-I Phasenanpassung) frequenzverdoppelt (ca. $\left.70 \mu \mathrm{J}\right)$ und als Pumppuls verwendet. Für die parametrische Erzeugung in der erste NOPA Stufe werden dazu etwa $20 \%$ des Pumppulses vom Strahlteiler (ST2) reflektiert. Die 
Frequenzverdoppelte bei $387 \mathrm{~nm}$ wird über einen sphärischen dielektrischen Spiegel (DS) in den NOPA-Kristall fokussiert (Typ-I-BBO, $5 \times 5 \times 1 \mathrm{~mm}^{3}, \Theta=32,5^{\circ}$ ) und mit dem Signalpuls überlagert. Da die verschiedenen Wellenlängenanteile des Kontinuums unterschiedliche Laufzeiten in den transmittiven, optischen Bauteilen besitzen, kommen unterschiedliche Wellenlängenanteile des Kontinuums zu unterschiedlichen Zeiten am NOPA-Kristall an (chirp). Welcher Wellenlängenbereich zeitlich mit dem Pumppuls überlagert wird, kann durch die Verschiebebühne am (ST2) eingestellt werden. Bei einem eingestellten NOPA-Kristallwinkel wird die Phasenanpassungsbedingung für einen breiten Spektralbereich des Signalpulses erreicht und so kann ein spektral breites Verstärkungsspektrum erzielt werden. Der Winkel zwischen Pump- und Signalpuls (NOPA-Winkel $\alpha \sim 6^{\circ}$ ) ist in der Vertikalen arrangiert, so dass der Signalpuls über dem Pumpspiegel (DS) hinwegläuft. Die zweite nachgeschaltete NOPA-Stufe dient nun zur Verstärkung des in der ersten Stufe generierten Signalpulses. Dazu werden die verbleibenden $80 \%$ des Pumpulses bei $387 \mathrm{~nm}$ in einem weiteren NOPA-Kristall (BBO, Typ-I, $\left.5 \times 5 \times 2 \mathrm{~mm}^{3}, \Theta=32,5^{\circ}\right)$ mit dem Signalpuls überlagert. Es ist möglich beide Stufen des NOPA spektral leicht versetzt zu betreiben, welches ein spektral noch breiteres Verstärkungsspektrum im Bereich von 460-700 nm (ca. 5-10 $\mu \mathrm{J}$ ) ermöglicht. Im Fourierlimit kann ein so erhaltenes Spektrum einer Pulsdauer im Sichtbaren von $<30 \mathrm{fs}$ entsprechen $[114,115]$. Um einen solch kurzen Puls zu generieren, muss der durch Dispersion in den optischen Elementen zeitlich stark verbreiterte Puls komprimiert werden. Die Kompression erfolgt mit einem Prismenkompressor [123].

Der p-polarisierte Puls aus dem NOPA wird über einen halben Spiegel (protected silver, $\mathrm{Ag}$ ) in den aus zwei Quarzprismen bestehenden Kompressor geleitet. Die Kompressoranordnung ist aus Platzgründen einmal über einen Spiegel (protected silver, Ag) gefaltet und wird höhenversetzt zweimal durchlaufen. Der Abstand zwischen den Prismen beträgt typischerweise $80-100 \mathrm{~cm}$. Nach Passieren des Kompressors überschreitet der Puls den halben Spiegel und trifft auf ein Periskop aus zwei Ag-Spiegeln, welches die Polarisation auf s-Polarisation dreht. Anschließend passiert der Strahl den Chopper (siehe Abschnitt 3.4) und wird schließlich über eine Plankonvexlinse (Quarz, $f=100 \mathrm{~mm}$ ) in einen BBO Kristall (Typ-I, $5 \times 5 \times 0,1 \mathrm{~mm}^{3}, \Theta=38^{\circ}$ bzw. $52^{\circ}$ ) fokussiert, in dem die zweite Harmonische im Bereich von $230-350 \mathrm{~nm}$ erzeugt wird. Der Strahl wird anschließend von einer weiteren Plankonvexlinse (Quarz, $f=100 \mathrm{~mm}$ ) wieder kollimiert. Das Abtrennen der p-polarisierten zweiten Harmonischen von der s-polarisierten Fundamentalen geschieht durch ein Glan-Taylor-Prisma (Alpha-Laser Technology) und zwei dielektrische Spiegel (BBHR, 280-380 nm, 45), die auf einer computergesteuerten Verschiebebühne (Physik Instrumente, PI M-415.DG) montiert sind. Der transmittierte Anteil der Fundamentalen durch den ersten Spiegel wird auf die Chopper-Photodiode (siehe Abschnitt 3.4.2) geleitet. Die reflektierte zweite Harmonische kann nun als Pumppuls für das Experiment verwendet werden. 


\subsubsection{Erzeugung des Probepulses im TOPAS}

Der TOPAS ist ein voll motorisierter, computergesteuerter optisch parametrischer Verstärker. Sein Prinzip beruht wie beim NOPA auf einem parametrischen Prozess. Jedoch wird beim TOPAS das Auftreten der spontanen parametrischen Erzeugung in einem nichtlinearen Kristall ausgenutzt. Falls die Energiedichte des Pumpstrahls im Kristall groß genug ist (einige $\mathrm{GW} / \mathrm{cm}^{2}$ ), kann simultan Signal- und Idlerwelle erzeugt werden. Dieses quantenstatistische Phänomen wird als Superfluoreszenz [124] bezeichnet. Im Gegensatz zu Optisch Parametrischen Oszillatoren (OPO) bedarf es beim TOPAS keines Oszillators. Der erzeugende Prozess wird TOPG (travelling wave optical parametric generation) genannt.

Die Einkoppelung der Laserfundamentalen wird über zwei dielektrische Spiegel (HR775 nm, 45 $)$ erreicht, welche nur einer geringen Justage bedürfen. Zentrales Element des TOPAS ist ein BBO-Kristall der insgesamt fünf mal vom Strahlengang passiert wird. Zwischen den beiden Einkopplungspiegeln werden etwa $90 \mu \mathrm{J}$ der Fundamentalen durch einen dielektrischen Strahlteiler abgetrennt, der zur späteren UVErzeugung in der Deep-UV-Einheit dient (s.u.). Dieser Anteil wird durch ein dem TOPAS identisches Gehäuse auf gleicher optischer Weglänge geführt. Von der in den TOPAS eintreffenden Fundamentalen wird durch zwei Strahlteiler ein wesentlicher Teil der Energie für die weiteren Verstärkerstufen im Kristall entnommen. Nur ein sehr geringer Teil trifft zum ersten Mal auf den Kristall der daraufhin schwache breitbandige Superfluoreszenz emittiert. Der zweite und vierte Durchgang durch den Kristall dienen zur Vorverstärkung der Superfluoreszenz, während der dritte Durchgang für die Verstärkung vernachlässigbar ist. Zwischen beiden Vorverstärkerstufen befindet sich ein optisches Gitter, welches den gewünschten Wellenlängenanteil aus der breitbandigen Superfluoreszenz selektiert. Während des fünften Kristalldurchgangs, der mit dem größten Anteil der Fundamentalen gepumpt wird, erhält der Strahl seine gewünschte Energie. Für den genaueren Aufbau des TOPAS sei auf [116] verwiesen.

Über den beschriebenen parametrischen Prozess werden Signalpulse (p-polarisiert) im Bereich von 1070-1560 nm und Idlerpulse (s-polarisiert) im Bereich von 1560-2790 nm mit einer Gesamtenergie pro Puls (Signal+Idler) von 25-100 $\mu \mathrm{J}$ generiert. Durch nachgeschaltete verschiedene nichtlineare Prozesse kann der Wellenlängenbereich von 190$1070 \mathrm{~nm}$ durchgestimmt werden:

- SHI: Zweite Harmonische des Idlerpulses, 770-1070 nm, s-polarisiert

- SHS: Zweite Harmonische des Signalpulses, 571-770 nm, s-polarisiert

- SFI: Summenfrequenz zwischen Idlerpuls und verbleibender Laserfundamentalen, $516-571 \mathrm{~nm}$, s-polarisiert 
- SFS: Summenfrequenz zwischen Signalpuls und verbleibender Laserfundamentalen, 450-516 nm, s-polarisiert

- FHI: Vierte Harmonische des Idlerpulses, 385-450 nm, p-polarisiert

- FHS: Vierte Harmonische des Signalpulses, 285-385 nm, p-polarisiert

- SH(SFI): Zweite Harmonische der SFI, 258-285 nm, p-polarisiert

- SH(SFS): Zweite Harmonische der SFS, 225-258 nm, p-polarisiert

- P+FHS: Summenfrequenz zwischen der Fundamentalen, die das Deep-UVGehäuse durchlaufen hat, und der FHS, 210-250 nm, s-polarisiert

- $\mathbf{P}+\mathbf{S H}(\mathrm{SF})$ : Summenfrequenz aus der Fundamentalen (Deep-UV) und SH(SFS) und $\mathrm{SH}(\mathrm{SFI}), 190-210 \mathrm{~nm}$, s-polarisiert

Das Abtrennen der gewünschten Wellenlänge von den übrigen Wellenlängen erfolgte über austauschbare Wellenlängenseparatoren (Light Conversion), die aus zwei parallel angeordneten dielektrischen Spiegeln bestehen. Für die in dieser Arbeit verwendeten Wellenlängen wurde z. B. die Wellenlängenseparatoren $\mathrm{HR}_{\mathrm{s}} 300-380 \mathrm{~nm} / \mathrm{HT}_{\mathrm{p}}$ 550-760 nm und $\mathrm{HR}_{\mathrm{s}}$ 380-480 nm / $\mathrm{HT}_{\mathrm{p}}$ 740-1000 nm verwendet. Zur Vorbereitung des Probestrahls für das Experiment und zur Kompensation der optischen Weglänge wird der Probestrahl über vier dielektrische Breitbandspiegel (BBHR 350-495 nm bzw. Aluminium-UV Spiegel bei $\lambda<355 \mathrm{~nm}$ ) durch ein Kepler-Teleskop mit zwei Plankonvexlinsen (Quarz, $f_{1}=100 \mathrm{~mm}$ und $f_{2}=80 \mathrm{~mm}$ ) geleitet, um den Strahldurchmesser zu verkleinern und die Divergenz zu kontrollieren. Ferner befindet sich im Fokus des Teleskops ein $150 \mu \mathrm{m}$ Pinhole als Fourier-Kurzpass-Raumfilter, um störende Raumfrequenzanteile des Pulses herauszufiltern. Da der TOPAS im Bereich der Laserfundamentalen und ihrer höheren Harmonischen nicht arbeitet, kann in einem BBO-Kristall (Typ-I, $5 \times 5 \times 0,3 \mathrm{~mm}^{3}, \Theta=29,18^{\circ}$ ), aus der das Deep-UV-Gehäuse passierenden Fundamentalen die zweite Harmonische mit $387 \mathrm{~nm}$ erzeugt und ebenfalls als Probewellenlänge genutzt werden.

\subsubsection{Das Pump-Probe-Interferometer}

Der schematische Aufbau des verwendeten Pump/Probe-Interferometers ist Abbildung $3.6 \mathrm{zu}$ entnehmen. Die zeitliche Verzögerung zwischen Pump- und Probepuls wird durch eine computergesteuerte Verschiebebühne realisiert (Physik Instrumente, PI M-415.DG), die in den Probestrahlengang eingebaut ist. Auf der Bühne befinden sich zwei dielektrische, hochreflektierende Breitbandspiegel (BBHR 350-495 nm), so dass sich eine maximale Verzögerung des hin- und zurücklaufenden Probestrahls von etwa 1 ns ergibt. Die rechnerische Auflösung der Bühne lässt sich mit 0,06 fs und die Reproduzierbarkeit mit 1,3 fs angeben. 


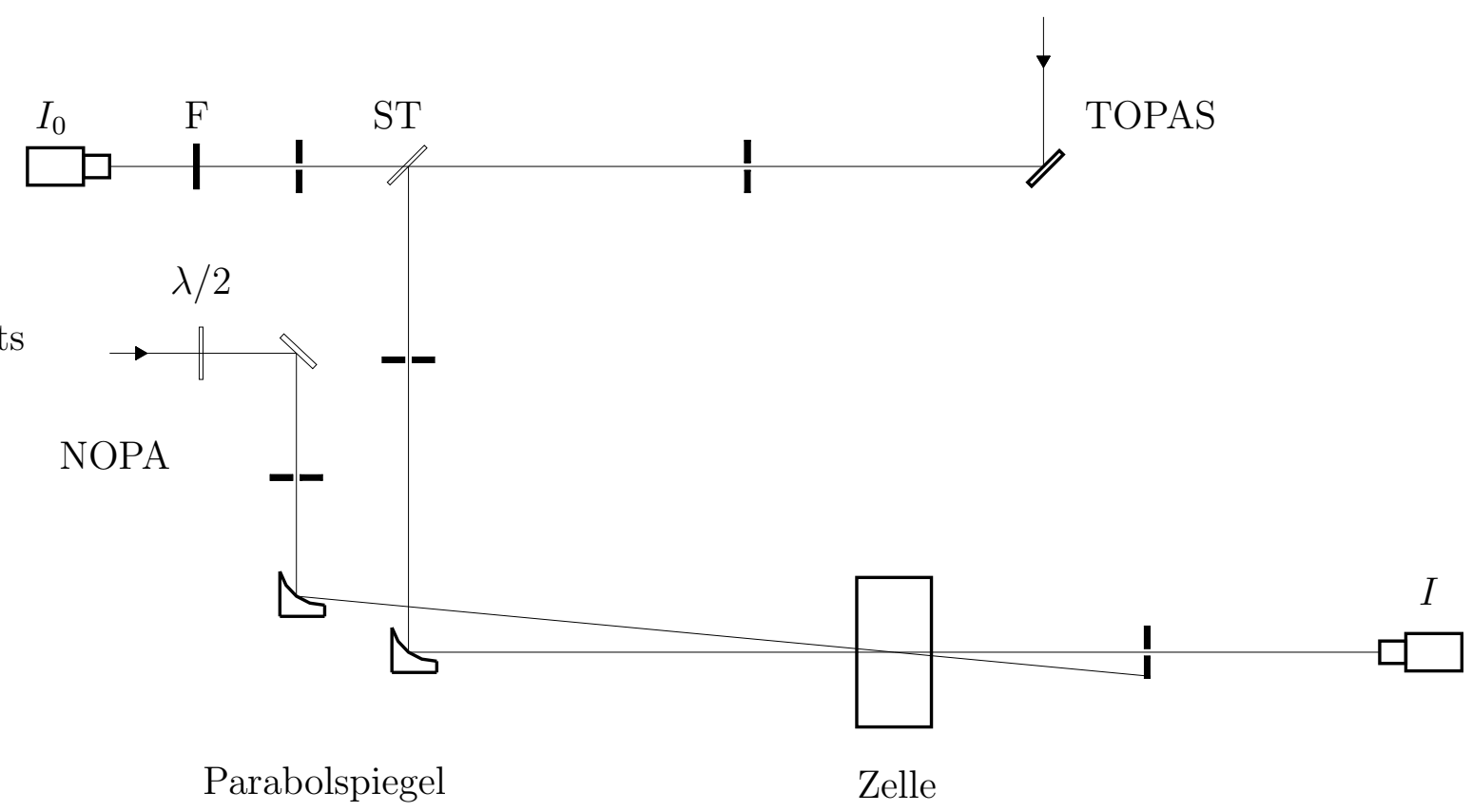

Abbildung 3.6: Schematische Darstellung des verwendeten Pump/Probe-Interferometers.

Der Probestrahl wird nach Passieren der Verschiebebühne über zwei weitere Spiegel auf einen dielektrischen Strahlteiler (Breitbandstrahlteiler $250-450 \mathrm{~nm}, R \approx 50 \%$ bei $320 \mathrm{~nm}, 45^{\circ}$, unpolarisiert) gelenkt. Der transmittierte Anteil $\left(I_{0}\right)$ wird von der Referenzphotodiode (HAMAMATSU, 1226-8BQ bzw. 1336-8BQ0A) detektiert, und der reflektierte Anteil wird auf einen Parabolspiegel (Janos Technology Inc., $90^{\circ}$ off-axis, protected aluminium, $f=152,4 \mathrm{~mm}$ ) gelenkt, von dem der Strahl in die Messzelle (siehe Abschnitt 3.5) fokussiert wird. Im Abstand von etwa $15 \mathrm{~cm}$ hinter der Messzelle befindet sich die Signalphotodiode (HAMAMATSU, 1226-8BQ bzw. 1336-8BQ0A), welche den von der Zelle transmittierten Anteil $(I)$ detektiert. Eine detaillierte Beschreibung der Signalaufnahme findet sich in Abschnitt 3.4. Der vom NOPA generierte Pumpstrahl geht bei Eintreffen in den Spektrometerbereich zunächst durch ein wellenlängenjustierbares $\lambda / 2$-Verzögerungsplättchen (Alphalas $\mathrm{GmbH}$, tunable true zeroorder phase retardation plate) für Femtosekundenanwendungen um den Winkel der Polarisationen zwischen Pump- und Probestrahl einszustellen. Anschließend wird der Strahl auf einen Parabolspiegel (Janos Technology Inc., $90^{\circ}$ off-axis, protected aluminium, $f=203,3 \mathrm{~mm}$ ) gelenkt, von diesem in die Messzelle fokussiert und mit dem Probestrahl räumlich überlagert. Der Winkel zwischen Pump- und Probestrahl in dieser Anordnung beträgt etwa $7^{\circ}$. Der Probefokus liegt in etwa im Zellmittelpunkt, wobei der Fokus des Pumpstrahls etwas hinter der Zelle liegt, um Beschädigungen der Zellfenster oder nichlineare Effekte in den Fenstern (z. B. Kontinuumserzeugung) zu vermeiden und um am Ort der Probe einen größeren Pumpstrahl- als Probestrahl- 
durchmesser zu gewährleisten. Die Durchmesser der Strahlen am Ort der Messzelle betragen typischerweiser $\sim 150 \mu \mathrm{m}$ für den Probe- und $\sim 300 \mu \mathrm{m}$ für den Pumpstrahl. Der kleinere Durchmesser des Probestrahls ermöglicht es, ein durch die Verschiebebühne verursachtes Herauslaufen des Probestrahls aus dem Pumpvolumen zu vermeiden. Ebenfalls wirken sich so lokale Intensitätsschwankungen innerhalb des Pumpstrahls nicht so stark auf das Signal- zu Rauschverhältnis aus. Die nichtkollineare Anordnung zwischen Pump- und Probestrahl ermöglicht es, nicht absorbiertes Pumplicht durch eine Blende abzutrennen. Ferner ist das Wechselwirkungsvolumen beider Pulse kleiner und ermöglicht eine bessere Zeitauflösung.

\subsection{Signalaufnahme}

In den folgenden Abschnitten soll das Verfahren zur Aufnahme der transienten Abklingkurven mittels des benutzten Pump/Probe-Spektrometers detailliert erklärt werden.

\subsubsection{Messprinzip}

Das grundlegende Prinzip der Pump/Probe-Experimente ist die Messung der Änderung der optischen Dichte $\triangle O D$. Die Messgröße bei diesem Verfahren ist der negative dekadische Logarithmus des Quotienten aus integraler Intensität pro Laserschuss auf der Signalphotodiode $(I)$ zur Referenzphotodiode $\left(I_{0}\right)$ nach dem Lambert-Beerschen Gesetz:

$$
O D=-\lg \left(\frac{I}{I_{0}}\right)
$$

Um die Absorptionsänderung $\triangle O D$ messen zu können, wird jeweils von der optischen Dichte eines Messwertes mit eingestrahltem Pumppuls $O D_{\text {auf }}$ die optische Dichte ohne Pumppuls $O D_{\mathrm{zu}}$ subtrahiert. Die Aufnahme beider optischer Dichten erfolgte alternierend, wobei jeder zweite Pumppuls durch den mit der Laserrepetitionsrate synchronisierten Chopper abgeblockt wird:

$$
\begin{aligned}
\triangle O D & =O D_{\text {auf }}-O D_{\mathrm{zu}} \\
& =\lg \left(\frac{I_{0, \text { auf }}}{I_{\text {auf }}}\right)-\lg \left(\frac{I_{0, \mathrm{zu}}}{I_{\mathrm{zu}}}\right) .
\end{aligned}
$$

Die Datenerfassung erfolgt mittels eines selbstgeschriebenen VEE (Agilent, VEE 6.1 pro [125])-Computerprogramms, welches einen Großteil der in Abschnitt 3.4.2 erklärten elektronischen Steuerung übernimmt. Das Signal der Photodioden liegt etwa zwischen 1 und $5 \mathrm{~V}$ und wird während der gesamten Messung mit einem Oszilloskop 
(Tektronix, TDS3052) überwacht. Ferner ist es für das Signal- zu Rauschverhältnis wichtig, dass die Diodensignale an Signalphotodiode $I$ und Referenzphotodiode $I_{0}$ in etwa die gleiche Höhe haben. Dies wird mit variablen Graufiltern zur Abschwächung direkt vor der Referenzphotodiode realisiert. Für jeden definierten Verzögerungszeitpunkt werden einstellbar zwischen 200 und 1000 Einzelintensitätsmessungen $\left(I_{0}, I\right)$ sowohl für Bedingungen mit Pump- als auch ohne Pumplicht aufgenommen. Als Entscheidungskriterium für geöffneten oder geschlossenen Chopper dient das Signal der Chopper-Photodiode, welches ebenfalls überwacht wird. Der Abstand zwischen zwei Verzögerungszeiten wird mit zunehmender Gesamtverzögerungszeit, von $50 \mathrm{fs}$ bis zu 50 ps, schrittweise vergrößert, um die Messzeit zu verkürzen. Für jede Messkurve werden in der Regel 6-8 Einzelspuren, entsprechend dreimaligem Vor- und Zurückfahren der Bühne, aufgenommen. Einzelmesswerte jedes Kanals $\left(I_{0}, I\right.$ für auf oder zu), die über einer Diskriminatorschwelle von $10 \%$ liegen oder Photodiodenspannungen, die unter $1 \mathrm{~V}$ liegen, werden als Ausreißer deklariert und verworfen. Über die verbleibenden 150-180 Messwerte werden die jeweiligen Mittelwerte errechnet und daraus die optischen Dichten $O D_{\text {auf }}$ und $O D_{\text {zu }}$ bestimmt. Das Grundrauschen der vom Computerprogramm aufgetragenen Absorptionsänderung $\triangle O D(t)$ kann bei 200 Einzelschussmittelungen auf $\triangle O D= \pm 4 \cdot 10^{-4}$ reduziert werden. Bei der Datenaufnahme im Computer werden eine Logbuchdatei, mit allen wichtigen Einstellungen des Messprogramms, und eine Datendatei im ASCII-Format erstellt. Die Datendatei enthält in ihren Spalten Daten zur Probewellenlänge, Verzögerungszeit, $\triangle O D$-Signal und Standardabweichung der Einzelintensitätsmessungen.

\section{Einfluss der Orientierungsrelaxation}

Bei Anregung der Probe mit linear polarisiertem Licht, wie in diesem Experiment, absorbieren vorzugsweise die Moleküle, deren Übergangsmatrixelement keinen oder nur einen kleinen Winkel $\psi$ mit dem elektrischen Feldstärkevektor bilden [126]. Nach Anregung mit linear polarisiertem Licht liegt also eine anisotrope Verteilung der angeregten Moleküle vor. Wenn der Feldstärkevektor für die transiente Absorption parallel zum Feldstärkevektor der Anregung liegt, kann in der Absorption ein Abklingen der Anisotropie gemessen werden. Über die Beobachtung von parallelem und senkrechtem Teil der Abfrage (Probepuls) kann eine Aussage über die Zeitabhängigkeit der Anisotropie und damit über die Zeitabhängigkeit der Umorientierung der Moleküle, der sogenannten Orientierungsrelaxation [127], gemacht werden. Die Zeitabhängigkeit der Anisotropie $r(t)$ kann über die folgende Beziehung beschrieben werden [126]:

$$
r(t)=\frac{A(t)_{\|}-A(t)_{\perp}}{A(t)_{\|}+2 A(t)_{\perp}},
$$

wobei $A(t)_{\|}$die transiente Absorption bei paralleler und $A(t)_{\perp}$ bei senkrechter Polarisation des Probepulses zum Pumppuls darstellt. Für die in Kapitel 4 vorgestellten 
Anisotropiemessungen wurden Abklingkurven mit senkrechter und paralleler Polarisation aufgenommen, um daraus die Anisotropie nach Gleichung $3.7 \mathrm{zu}$ berechnen.

Um den Beitrag der Orientierungsrelaxation der Moleküle zu unterdrücken, wurde bei allen anderen Messungen der magische Winkel von $\psi=54.7^{\circ}[128,129]$ eingestellt. Die Einstellung des Winkels zwischen Pump- und Probepuls erfolgt mit dem in Abschnitt 3.3.4 beschriebenen $\lambda / 2$-Verzögerungsplättchen.

\subsubsection{Elektronische Steuerung}

Der schematische Aufbau der elektronischen Steuerung findet sich in Abbildung 3.7. Eine schnelle Photodiode (PD) in der unteren Sektion des CPA-2001 (siehe Abschnitt 3.3.1) nimmt in Form von Streulicht direkt die Repetitionsrate des Faserlasers auf. Ihr Signal wird in den RF-IN-Eingang des Hochfrequenztreibers DT-505 [130] eingespeist. Über den RF-OUT-Anschluss kann das Signal direkt weiter verwendet werden. Die Frequenz des internen Signals wird über zwei Divider um den festen Faktor 4 und einen Faktor, hier 12, geteilt. Es wird eine Frequenz von etwa $1 \mathrm{kHz}$ erhalten. Dieses geht über ein kurzes BNC-Kabel in den DELAY-IN-Eingang des zweiten Teils des DT-505. Der ZERO-DELAY-OUT wird als Trigger des Q-Switch des ORC1000-Nd:YAG-Pumplaser verwendet. Das Signal, welches über den DELAY1 verzögert wurde, wird zum Schalten des Hochspannungsschalters HV1 der Pockelszelle und so zum Einkoppeln des Seedpulses in die Verstärkerkavität verwendet. Der über DELAY2 verzögerte Teil bewirkt über den zweiten Hochspannungsschalter HV2 das Auskoppeln der Pulse mittels der Pockelszelle.

Ein parallel abgegriffener Teil des $1 \mathrm{kHz}$-Signals aus der ersten Stufe des DT-505 wird als Eingangspuls in einen Delay-Generator (DG-535, Stanford Research Systems Inc.) gegeben. Ein nichtverzögerter Ausgang des DG-535 triggert die Steuerung des Choppers, der so mit den ausgekoppelten Laserpulsen synchronisiert wird. Ein weiterer, verzögerter Ausgang triggert die Steuereinheit der Photodioden und löst in den Integratorschaltungen [131] der Photodioden den Start der Integration aus. Die Integrationszeit lässt sich dabei direkt über ein Potentiometer an der Photodiodensteuerung einstellen und kann im Bereich von 90-1800 $\mu$ s variiert werden. Ausgelesen werden die Signale der Photodioden über 12 Bit-AD-Wandlereingänge einer ISA-Bus-AD/DA-Karte (Data Translation, DT2821) (AD), die sich in einem Personalcomputer (PC) befindet. Die Synchronisation der Wandlerkarte ist durch einen weiteren verzögerten Ausgang des DG-535 gegeben, der den TRIGGER-IN-Kanal der Karte speist. Vom PC aus kann über eine PCI-Bus-IEEE488.2-Interfacekarte die Steuereinheit (Physik Instrumente, PI C-844) (C-844) der Verschiebebühnen (Bühne) betrieben werden und Bühnenparameter, wie z. B. Position oder Geschwindigkeit, gesetzt und abgefragt werden. Desweiteren lässt sich der TOPAS über die interne Parallelport-Schnittstelle (Par) steuern, 


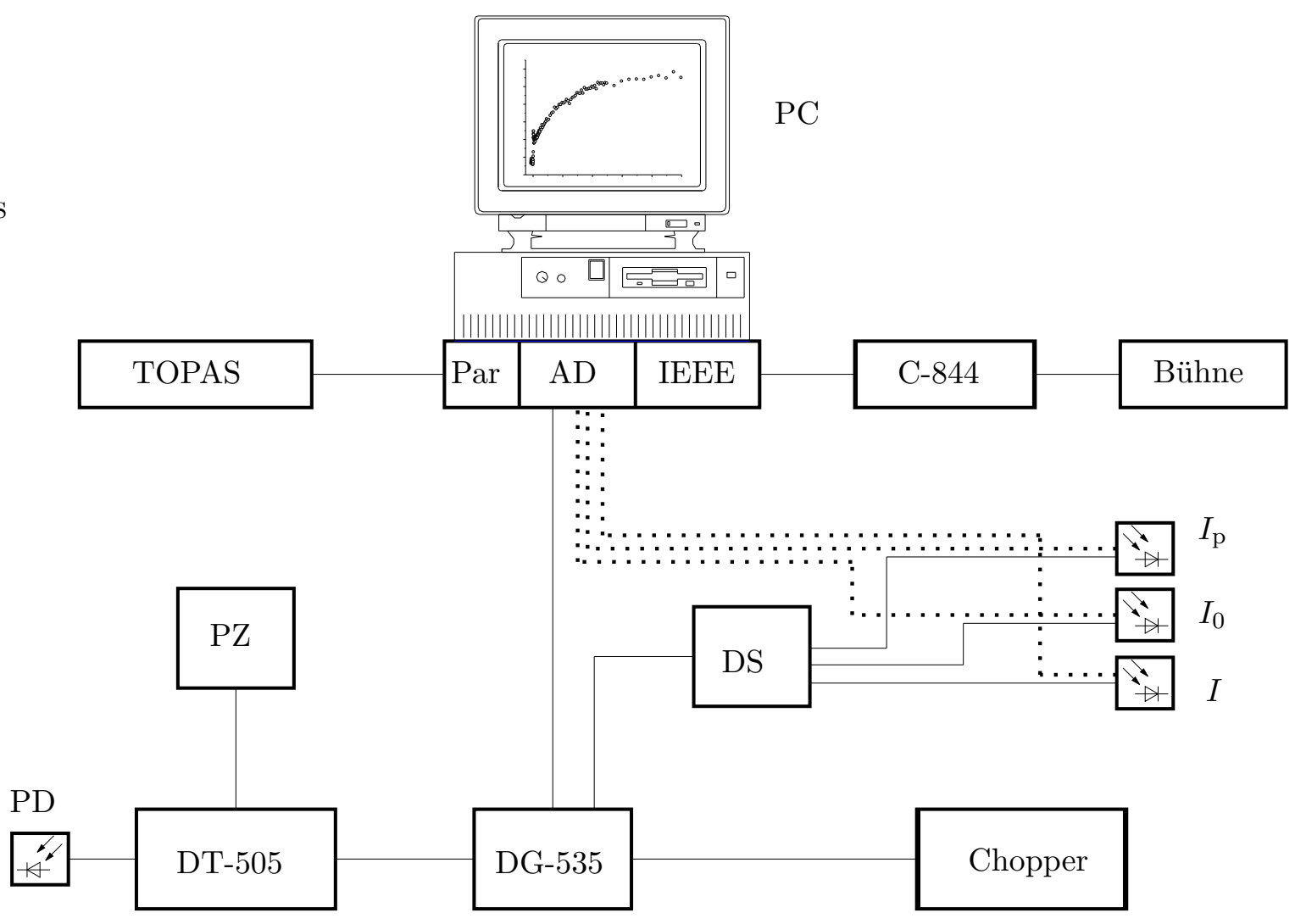

Abbildung 3.7: Schematische Darstellung der elektronischen Steuerung des Experimentes.

um so die gewünschte Wellenlänge zu selektieren. Alle genannten Steuer- und Datenaufnahmemöglichkeiten des PC sind in das selbstgeschriebene Computerprogramm integriert.

\subsubsection{Signalauswertung}

Zur Analyse und Auswertung der Rohdaten wird ein selbstgeschriebenes MatlabProgramm (Mathworks, Matlab 6.1.0.450 Release 12.1) mit GUI (graphical user interface) verwendet. Das Programm besteht aus 3 Teilen, dem Fitprogramm „fitte“, dem Mittelungsprogramm „mittelmich“ und dem Optimierungsprogramm „optimize“. Die Rohdaten (z. B. ch2i20032. dat) des Messprogramms können in das Mittelungsprogramm eingelesen werden. Dort werden sie zunächst der Probewellenlänge nach in einzelne Dateien aufgeteilt und die Probewellenlänge an den Dateinamen angehängt (z. B. ch2i20032_405. dat). Die Rohdateien werden graphisch dargestellt und das Programm mittelt die Rohdaten über die aufgenommenen Einzelspuren und verwirft ein- 
stellbar alle $\triangle O D$-Punkte die mehr als 1-3 Standardabweichungen vom Mittelwert bei einem Verzögerungszeitpunkt entfernt liegen. Die gemittelte Datei wird mit einem weiteren Anhang an den Dateinamen versehen (z. B. ch2i20032_405m.dat).

Zentrales Element des Auswertungsprogramms ist das Fitprogramm. In diesem wird die gemittelte Datei eingelesen und ebenfalls wieder graphisch dargestellt. Es können nun u. a. die entsprechende Fitfunktion, (z. B. Monoexponentieller Abfall + Monoexponetieller Anstieg (siehe Gleichung 3.11); andere wählbare Fitfunktionen siehe Anhang 6.7 auf Seite 121), die Startparameter und der zu verwendende Algorithmus (meist Levenberg-Marquardt) ausgewählt werden. Die Fitfunktion $f(t)$ besteht aus einer Modellfunktion $e(t)$, die analytisch mit einer, der zeitlichen Breite der Kreuzkorrelation der Laserpulse entsprechenden, Gaußunktion $g(t)$ gefaltet wird:

$$
\begin{aligned}
& f(t)=(g \otimes e)(t)=\int_{0}^{\infty} g(t) \cdot e(t+\tau) \mathrm{d} \tau \text { mit } \\
& g(t)=\frac{1}{2 \pi \sigma^{2}} \exp \left(-\frac{t^{2}}{2 \sigma^{2}}\right)
\end{aligned}
$$

Für die Analyse der Daten wurde meist die Fitfunktion mit monoexponetiellem Anstieg und Abfall benutzt:

$$
\begin{aligned}
e(t) & =A \cdot e_{1}(t)+B \cdot e_{2}(t)+I_{0} \cdot e_{3}(t) \\
e_{1}(t) & =\exp \left(-\frac{t}{\tau_{1}}\right) ; e_{2}(t)=1-\exp \left(-\frac{t}{\tau_{2}}\right) ; e_{3}(t)=1 \\
g(t) & =\frac{1}{2 \pi \sigma^{2}} \exp \left(-\frac{\left(t-t_{0}\right)^{2}}{2 \sigma^{2}}\right) .
\end{aligned}
$$


Daraus folgt für die Fitfunktion:

$$
\begin{aligned}
f(t) & =A \cdot \int_{0}^{\infty} g(t) \cdot e_{1}(t+\tau) \mathrm{d} \tau+B \cdot \int_{0}^{\infty} g(t) \cdot e_{2}(t+\tau) \mathrm{d} \tau \\
& +I_{0} \cdot \int_{0}^{\infty} g(t) \cdot e_{3}(t+\tau) \mathrm{d} \tau+I_{1} \\
& =A \cdot \exp \left(\frac{\sigma^{2}-2\left(t-t_{0}\right) \tau_{1}}{2 \tau_{1}^{2}}\right) \cdot \sqrt{\frac{\pi}{2}} \sigma\left(1+\operatorname{erf}\left(\frac{-\sigma^{2}+\left(t-t_{0}\right) \tau_{1}}{\sqrt{2} \sigma \tau_{1}}\right)\right) \\
& +B \cdot \exp \left(\frac{-\left(t-t_{0}\right)}{2 \tau_{2}^{2}}\right) \cdot \sqrt{\frac{\pi}{2}} \sigma \cdot \exp \left(\frac{t-t_{0}}{\tau_{2}}\right) \cdot\left(1+\operatorname{erf}\left(\frac{t-t_{0}}{\sqrt{2} \sigma}\right)\right) \\
& +\exp \left(\frac{\sigma^{2}}{2 \tau_{2}^{2}}\right) \cdot\left(-1+\operatorname{erf}\left(\frac{\sigma^{2}-\left(t-t_{0}\right) \tau_{2}}{\sqrt{2} \sigma \tau_{2}}\right)\right) \\
& +I_{0} \cdot \sqrt{\frac{\pi}{2}} \sigma\left(1+\operatorname{erf}\left(\frac{t-t_{0}}{\sqrt{2} \sigma}\right)\right)+I_{1},
\end{aligned}
$$

mit den Fitparametern:

- $A, B, C$ : Amplituden

- $t_{o}$ : Zeitnullpunkt

- $\tau_{1}, \tau_{2}$ : Anstiegs- bzw. Abfallzeitkonstante

- $\sigma$ : halbe Halbwertsbreite der Gaußfunktion (Kreuzkorrelation)

- $I_{0}$ : Endoffset

- $I_{1}$ : Gesamtoffset.

Die eigentliche Fitroutine greift auf die Curve-Fitting-Toolbox (Matlab Curve Fitting Toolbox 1.0 Release 12.1), ein Matlab-Zusatzpaket, zurück. Die angepaßten Parameter, die Fitgüte (z. B. $R^{2}$ ) sowie andere wichtige Eingabedaten werden vom Programm ausgegeben und in eine Datei abgespeichert (z. B. ch2i20032_405m.out). Ferner werden noch die angepasste Kurve mit den anzupassenden Daten und die Residuen gemeinsam graphisch dargestellt. Für angepasste Kurve (z. B. ch2i20032_405m.sim) und Residuen (z. B. ch2i20032_405m.res) werden ebenfalls Dateien angelegt. Das Fitprogramm ermöglicht es außerdem jeden beliebigen Parameter als Konstante zu setzen und nicht mit anzupassen. Dieses eröffnet eine sukzessive Methode, mit bekannten Größen eine gute Anpassung zu erhalten. Die so gewonnenen Anpassungsparameter werden in eine separate Datei (z. B. ch2i20032_405m.par) gespeichert und können direkt für die nächste Anpassung als Startparameter verwendet oder zu anderer Zeit wieder in das 


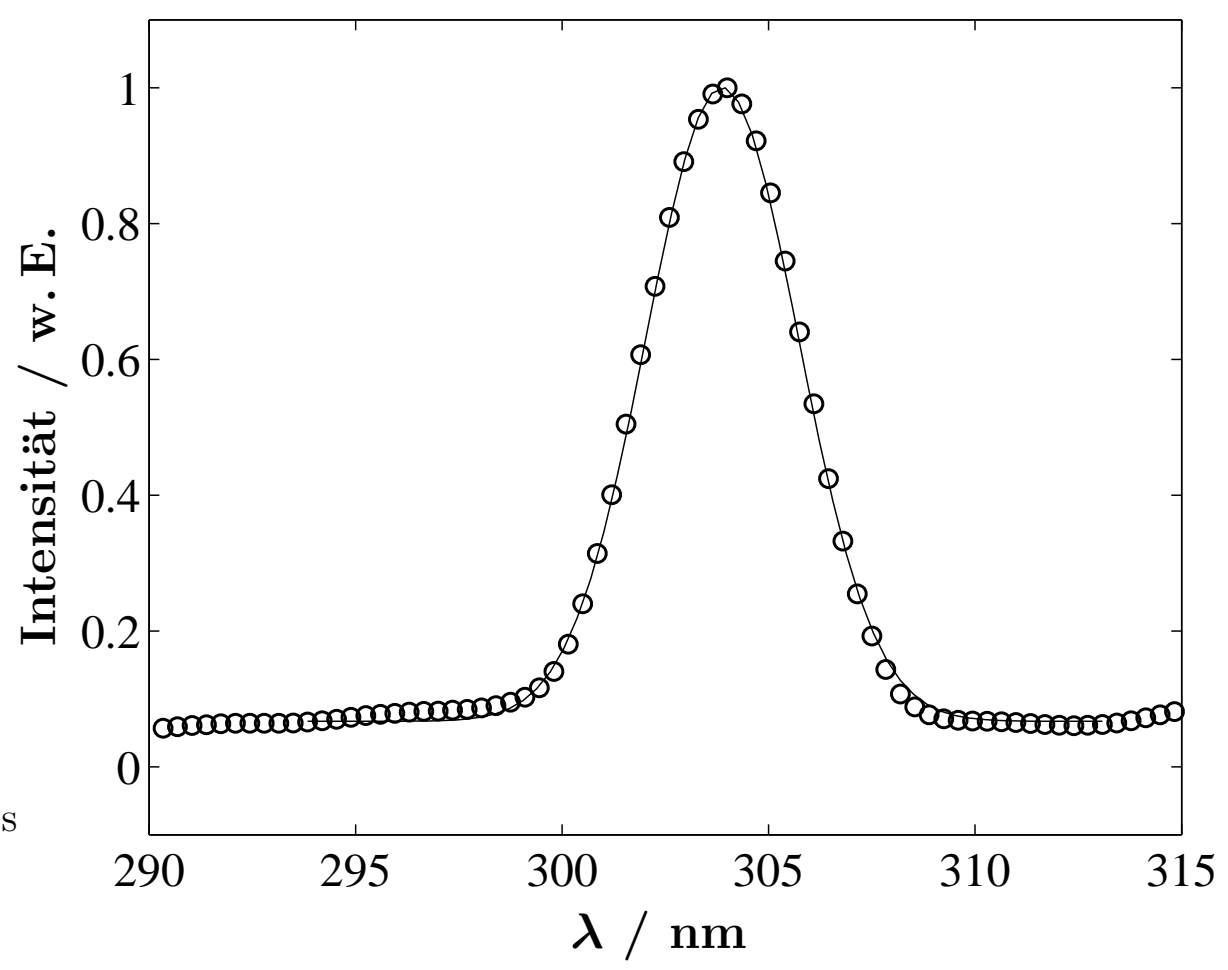

Abbildung 3.8: Spektrum der Anregungspulse bei einer Zentralwellenlänge von $304 \mathrm{~nm}$. Die durchgezogene Linie zeigt eine gauß̈örmige Anpassung mit einer Halbwertsbreite von $3,8 \mathrm{~nm}$.

Programm geladen werden. Das Programm „optimize“ bietet eine Möglichkeit gute Startparameter für die Anpassung zu finden. Es zeigt den Graph der Fitfunktion mit gegebenen Startparametern an die anzupassenden Daten.

\subsubsection{Charakterisierung des Messsystems}

\section{Spektrale Auflösung der Pump- und Probepulse}

In Abbildung 3.8 findet sich ein typisches Spektrum der Pumppulse bei einer Zentralwellenlänge von $304 \mathrm{~nm}$ und einer spektralen Halbwertsbreite von etwa $7 \mathrm{~nm}$ (bei gaußförmiger Anpassung, durchgezogene Linie). Nach jeder neuen Justage des NOPA wird ein Spektrum des Anregungspulses aufgenommen und die Zentralwellenlänge sowie die Halbwertsbreite bestimmt. Die spektrale Breite der Pulse im Bereich von 250-320 nm liegt zwischen 5 und $8 \mathrm{~nm}$. Die Spektren der Pump- und Probepulse werden mit einem Glasfaser-Spektrometer (AVANTES, AVS-SD2000) gemessen. Als Beispiele für die spektrale Auflösung des TOPAS sind in Abbildung 3.9 einige Probespektren dargestellt. Die Eichung der Wellenlängen des TOPAS wurde bei der Erstinstallation des 


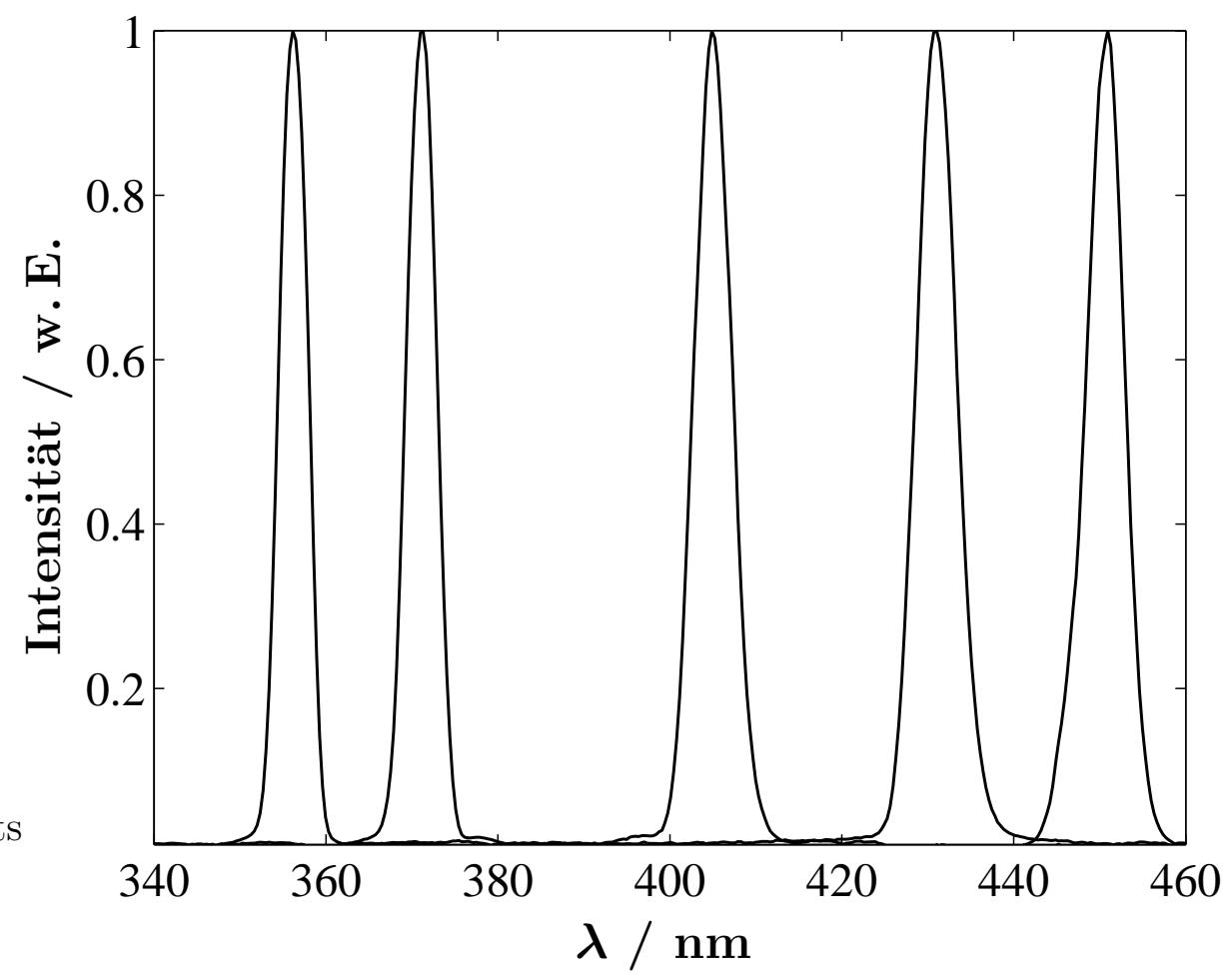

Abbildung 3.9: Beispielspektren der Abfragepulse bei verschiedenen Zentralwellenlängen.

Gerätes vorgenommen und stimmte über den gesamten zur Verfügung stehenden Wellenlängenbereich stets auf 1-2 nm mit den im Spektrometer gemessenen Wellenlängen überein.

\section{Zeitliche Auflösung des Spektrometers}

Die zeitliche Auflösung des Pump/Probe-Spektrometers kann mit etwa 150-300 fs angegeben werden. Im Wesentlichen wird die zeitliche Auflösung durch das Auswerteprogramm (siehe Kapitel 4) erhalten, welches eine gaußförmige Kreuzkorrelation des Pump- und Probepulses in der Signalauswertung berücksichtigt. In Zusammenarbeit mit Dr. Matthias Kling [108] wurde die Kreuzkorrelation durch transiente Absorption in reinem Propylencarbonat in einer Durchflussküvette bei einer Anregungswellenlänge von $266 \mathrm{~nm}$ und einer Probewellenlänge von $310 \mathrm{~nm}$ unter Annahme eines gaußförmigen Zeitprofils zu 220 fs ermittelt (siehe Abbildung 3.10). 


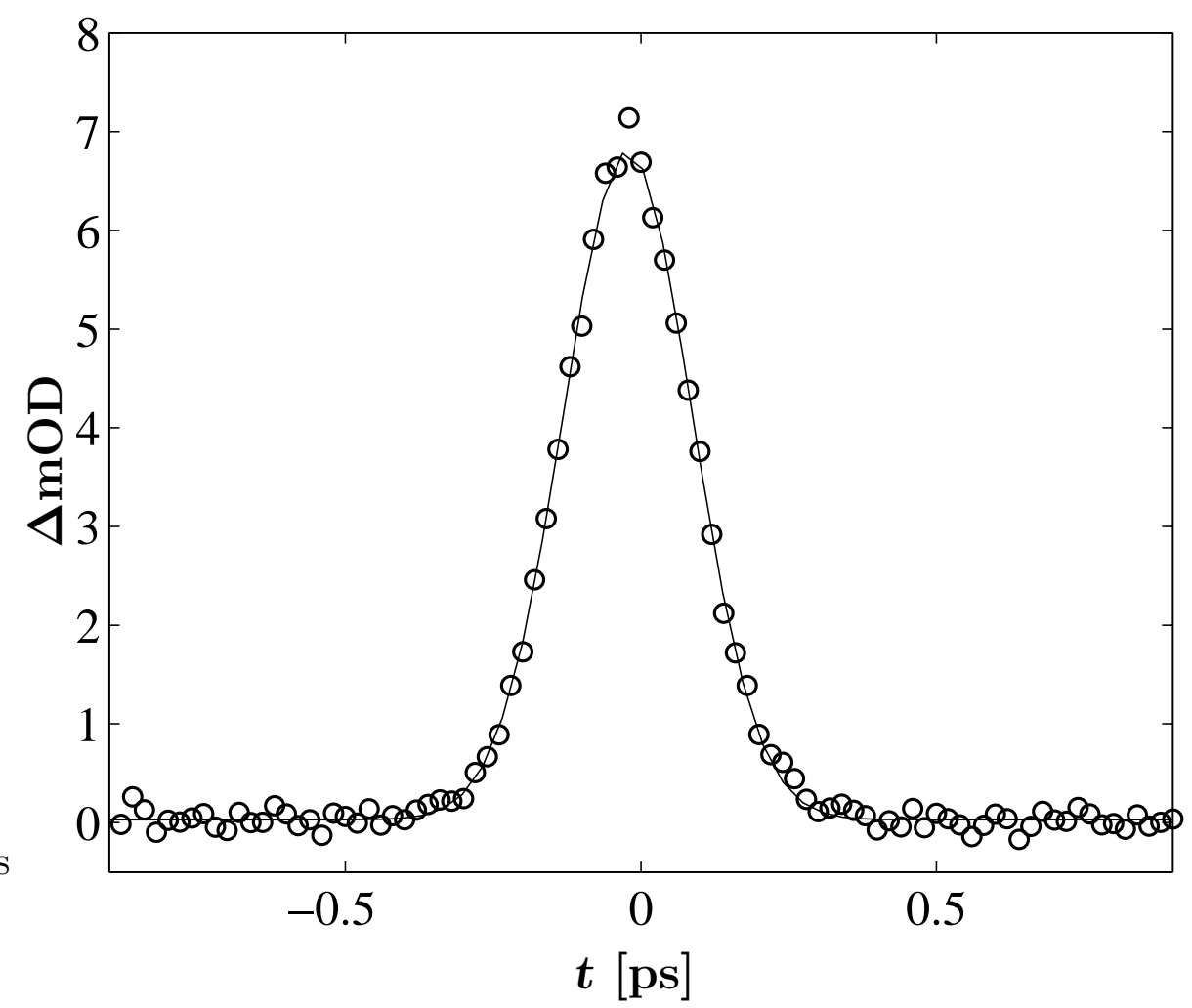

Abbildung 3.10: Transiente Absorption bei $310 \mathrm{~nm}$ nach Anregung mit $266 \mathrm{~nm}$ in Propylencarbonat aus [108]. Die durchgezogene Linie entspricht einer gaußförmigen Anpassung mit einer Halbwertsbreite von 220 fs.

\subsection{Aufbau und Funktion der Messzelle}

\section{Durchflusszelle}

Für die Voruntersuchungen in der flüssigen Phase stand eine Durchflussmesszelle zur Verfügung, die von der feinoptischen Werkstatt des Max-Planck-Institutes für Biophysikalische Chemie konzipiert und hergestellt wurde [132]. Auf einen Quarzspiegelrohling (Suprasil, $\varnothing=25,4 \mathrm{~mm}$, Dicke $=6,25 \mathrm{~mm}$ ) ist ein etwa $4 \mathrm{~mm}$ breiter und $0,19 \mathrm{~mm}$ dicker Abstandsring aus Quarz aufgesprengt. Auf diesen wiederum ist ein poliertes 0,2 mm dickes Quarzfenster aufgeklebt. Zwei seitliche Bohrungen, die zum Zellinneren abgewinkelt sind nehmen zwei Anschlussstutzen aus VA-Stahl, die als Ein- und Auslass dienen, auf. Die Anschlüsse sind z. B. über einen PTFE-Schlauch und eine Laserfarbstoffpumpe (Micropump Corporation) mit einer Vorratsflasche verbunden. In der Vorratsflasche befinden sich etwa $50 \mathrm{ml}$ Messlösung. Die Messzelle selbst ist zur Justage auf einen verstellbaren Spiegelhalter aufgeklebt. 


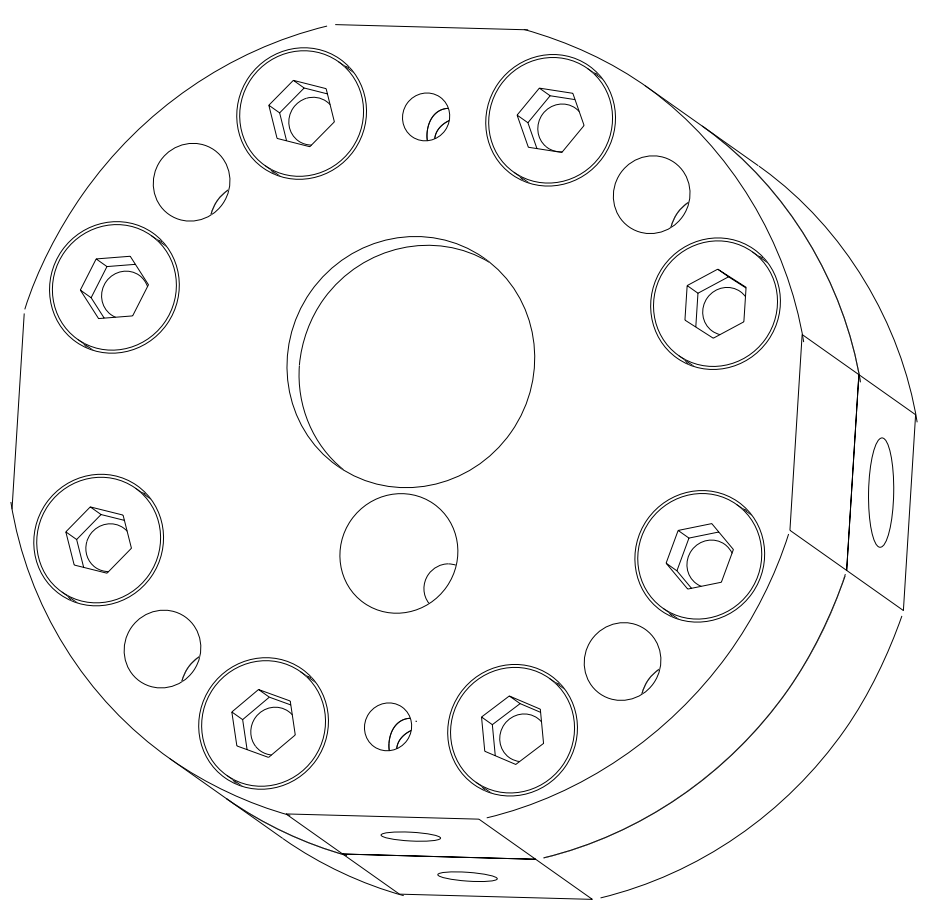

Abbildung 3.11: Gesamtansicht der verwendeten Hochdruckmesszelle.

\section{Hochdruckzelle}

Die verwendete Hochdruckzelle ist in Konstruktion und Design einer Hochdruckzelle der Arbeitsgruppe Kajimoto [133] angelehnt. Sie wurde in Zusammenarbeit mit der Arbeitsgruppe Schwarzer $[134,135]$ von der feinmechanischen Werkstatt der Abteilung 010 des MPI f. biophys. Chemie (MPIBPC) gefertigt [136]. Eine weitere Umarbeitung der Anschlüsse erfolgte durch die feinmechanische Werkstatt des Instituts für Physikalische Chemie (IPC) der Universität Göttingen [137].

Die Zelle (siehe Abbildung 3.11 und 3.12) hat einen Außendurchmesser von $68 \mathrm{~mm}$ und besteht aus hochfestem Spezialstahl (Inconel Alloy 718, Materialnr. 2.4668). Sie ist in zwei $21 \mathrm{~mm}$ dicke Hälften unterteilt. In beide Hälften ist eine 3,5 $\mathrm{mm}$ Vertiefung als Substanzreservoir eingedreht. Das so erhaltene Innenvolumen, bei eingesetztem Magnetrührer) wurde mit dem 3D-Designprogramm Inventor (Autodesk, Inventor Series 6) zu $2,59 \mathrm{~cm}^{3}$ berechnet. In dem Reservoir findet ein drehbarer Zylinder Platz, der einen Magnetrührstab (Bola, $\varnothing=4,5 \mathrm{~mm}, l=15 \mathrm{~mm}$, PTFE-beschichtet) aufnimmt. Der Magnetrührstab kann von einem außerhalb der Vorderseite der Zelle angebrachten motorisierten drehbaren Magneten bewegt werden und sorgt so für ein ständiges Umwälzen der Lösung im Reservoir. Oberhalb der Vertiefungen befindet sich jeweils eine etwa $2 \mathrm{~mm}$ tiefe Fensteraufnahme $(\varnothing=10 \mathrm{~mm})$. In diese werden $2 \mathrm{~mm}$ dicke Saphirfenster (Korth, $\varnothing=10_{-0,1}^{+0} \mathrm{~mm}$, optisch poliert, senkrecht zur C-Achse geschnitten) mit 

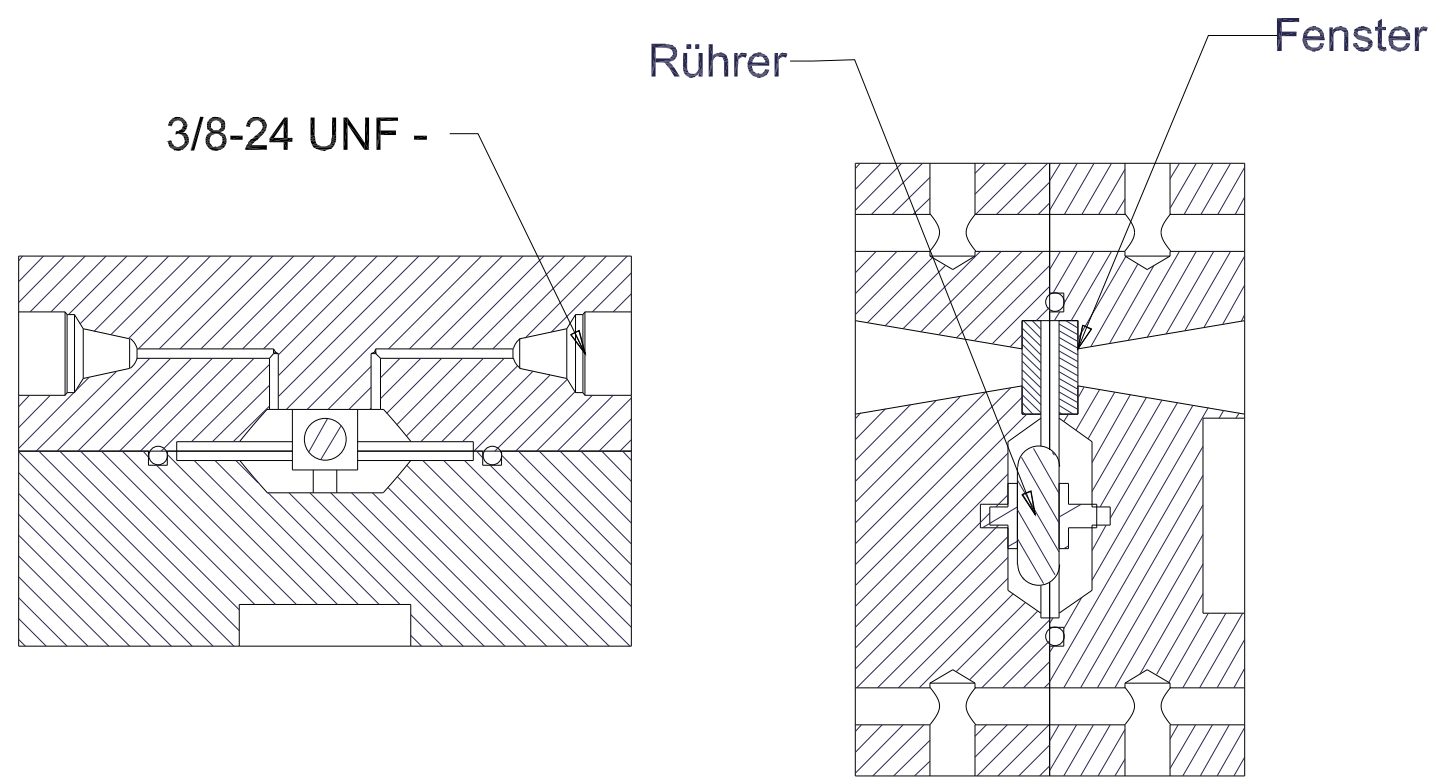

Abbildung 3.12: Schnittansicht der verwendeten Hochdruckmesszelle.

2 Komponentenkleber (UHU Plus Endfest 300) eingeklebt. Die optisch freie Apertur der Zelle beträgt an der Außenseite $10 \mathrm{~mm}$ und läuft konisch auf $4 \mathrm{~mm}$ zum Zelleninneren zu. Die freie optische Weglänge in der Zelle zwischen den Fenstern beträgt etwa 2,5 mm. Die Zelle wird mit einem PTFE-Dichtring $34 \times 2 \mathrm{~mm}^{2}$ (wahlweise auch Viton) abgedichtet, der in einer in die Vorderseite gefrästen Nut liegt. Zusammengehalten wird die Zelle von acht ebenfalls aus hochfestem Stahl gefertigten M6-Inbus-Schrauben.

Im Zellkörper befinden sich zusätzlich noch vier radialsymmetrische Bohrungen mit einem Durchmesser von 6,5 mm und Verschraubungen an der Rückseite zur Aufnahme von vier Heizpatronen (HotSet GmbH, HHP 6,5×40 mm², $125 \mathrm{~W}, 230 \mathrm{~V}$ ). Diese ermöglichen ein Temperieren der Zelle über eine, von der Elektronikwerkstatt [138] des Inst. f. Phys. Chemie umgebaute, Heizsteuerung. Das Zentralelement dieser Steuerung ist ein auf $\pm 0,5^{\circ} \mathrm{C}$ genauer Temperaturregler (Eurotherm), der die Temperatur über einen PT-100-Widerstandstemperaturmesskopf ausliest. Der PT-100-Messkopf ist dabei über eine 3,1 mm große Bohrung im Zellkörper in die Zelle eingebracht. Die experimentell mögliche Höchsttemperatur bei eingeklebten Fenstern liegt bei etwa $45^{\circ} \mathrm{C}$.

Als Anschlüsse an das in Abschnitt 3.6 beschriebene Hochdrucksystem werden 1000 bar Laborverschraubungen (NOVA Swiss, 3/8"-24UNF) für 1/16"-Hochdruckkapillaren benutzt. Ferner ist die gesamte Zelle mit zwei Hälften aus bearbeitbarer Glaskeramik (Corning, Macor) ummantelt. Diese dienen als Wärmeisolierung und an der Vorderseite als Aufnahme für den Motor, der den Magneten dreht. 


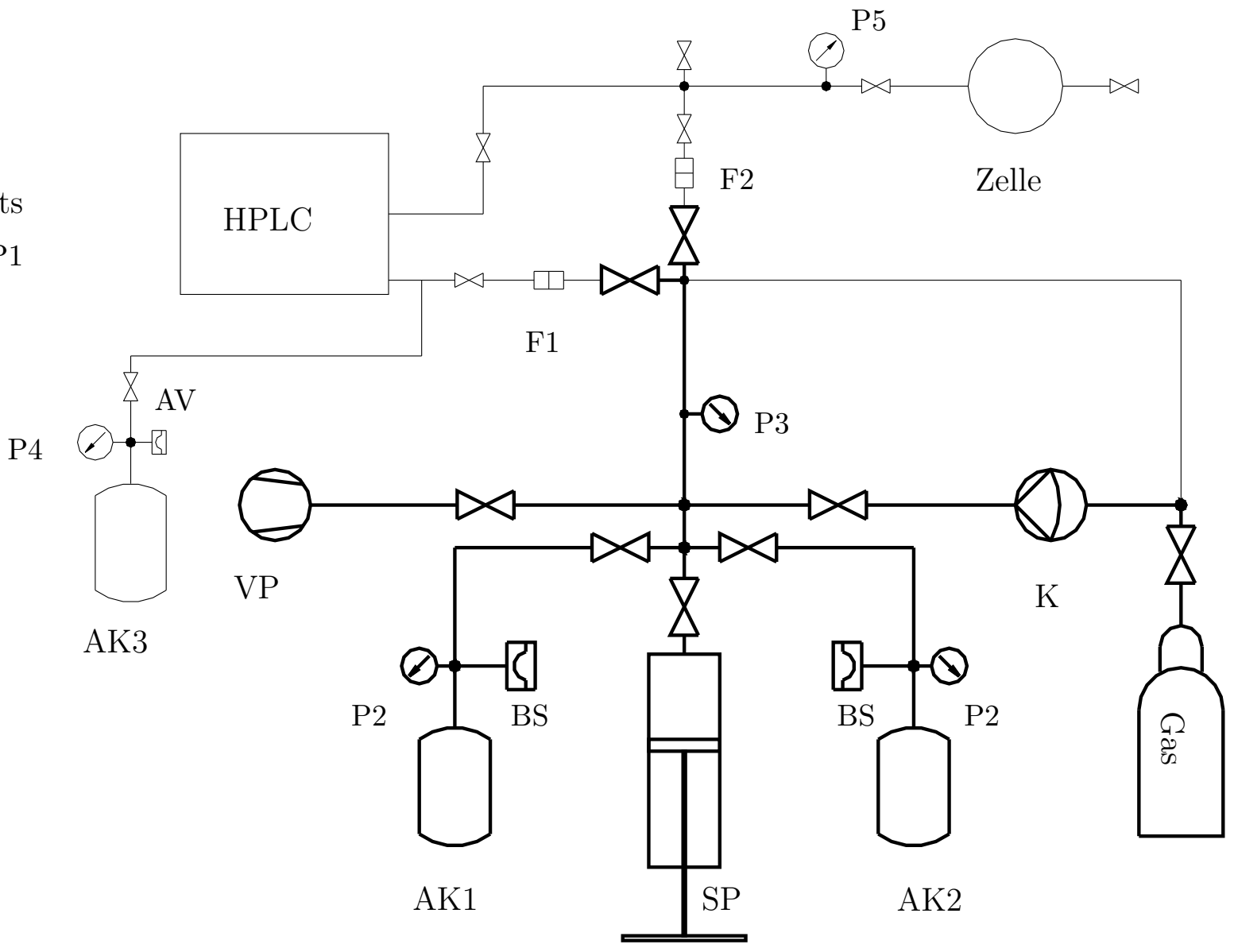

Abbildung 3.13: Schematische Ansicht des verwendeten Hochdrucksystems.

\subsection{Verwendetes Hochdrucksystem}

Eine schematische Zeichnung des verwendeten Hochdruckstandes zeigt Abbildung 3.13. Das gezeigte Hochdrucksystem lässt sich in zwei Bereiche unterteilen. Einen Messzellenbereich (dünne Linien) rund um die Zelle, in dem als Leitungen ausschließlich 1/16"Kapillaren (Techlab GmbH, Edelstahlkapillare, Durchmesser 0,25 mm, 1000 bar) zur Anwendung kommen. Die dort verwendeten Absperrventile (NOVA Swiss, Laborventil, LHP 1/16", TYP1) sind ebenfalls bis 1000 bar ausgelegt. Im anderen Bereich, dem Vorratsbereich (dickere Linien), werden als Leitungen 1/4"-Kapillaren (NOVA Swiss, 4000 bzw. 7000 bar) und entsprechende NOVA-Swiss-Absperrventile verwendet.

Zur Befüllung des Vorratsbereiches wird ein von der Hauspressluft betriebener Kompressor (K) (NOVA Swiss, Membrankompressor, pneumatisch betrieben, 1000 bar, ölfrei) benutzt. Dieser wird über eine Steuerung bei einem gewünschtem Druck abgeschaltet. Die Steuerung wird mit einem dem Druck äquivalenten Spannungssignal eines 
Druckanzeigegerätes gespeist. Der zugehörige Drucksensor (P1) (Burster, Präzisionsdrucktransmitter 8201-6001, 0-1000 bar) befindet sich direkt hinter dem Kompressor. Mittels des Kompressors können sowohl das gesamte System als auch wahlweise die beiden Autoklaven (AK1/AK2) (Mechanikwerkstatt IPC, Selbstbau, $P_{\max }=500$ bar) sowie die Spindelpresse (SP) (Mechanikwerkstatt IPC, Selbstbau, $P_{\max }=4000$ bar) befüllt werden. Beide Autoklaven verfügen über ein angeschlossenes Druckdosenmanometer (P2) $\left(P_{\max }=400 \mathrm{bar}\right)$ und sind jeweils über eine Berstscheibe (BS) (Nova Swiss, 450-550 bar) gesichert. Die Auslassöffnungen der Berstscheiben sind zum Splitterschutz zusätzlich mit einem nach unten offenen Kupferrohr gesichert. Alternativ kann der Vorratsbereich auch direkt über den Anschluss einer Druckgasflasche (Gas) befüllt werden. Der Druck im Vorratsbereich wird durch einen weiteren Drucksensor (P3) (NOVA Swiss, 0-7000 bar) überwacht. Zur Reinigung und Belüftung des Vorratsbereiches steht eine Vakuumpumpe (VK) (Edwards, Speedivac) zur Verfügung.

Der Druckaufbau im Messzellenbereich ist direkt aus dem Vorratsbereich mittels Spindelpresse oder Kompressor zu bewerkstelligen, kann aber auch über eine umgebaute, elektronisch gesteuerte HPLC-Pumpe (HPLC) (Techlab GmbH, Economy 2/ED) erreicht werden. Letztere eignet sich besonders für geringe Gasmengen, wie bei teurem Xenon. Der Einlass in die Pumpe ist über einen In-Line-Filter (F1) (LATEK, Porengröße $0,2 \mu \mathrm{m})$ direkt aus dem Vorratsbereich oder über einen weiteren transportablen Autoklaven (AK3) (HOKE, 4HS300, $300 \mathrm{ml}, P_{\max }=124$ bar) mit direkt folgendem Druckdosenmanometer (P4) und Abblassicherung (AV) möglich. Die Direktbefüllung aus dem Vorratsbereich geschieht ebenfalls über einen In-Line-Filter (F2) (GAT, 1/16", 0,25 mm Bohrung mit $2 \mu \mathrm{m}$ screens), um Verschmutzungen zu vermeiden. Der Druck in der Messzelle wird über ein Piezomanometer (P5) (Keller GmbH, PA-8, 0-1000 bar) überwacht, welches mit einer Vitondichtung abgedichtet ist.

\subsection{Aufnahme stationärer Absorptionsspektren}

Zur Messung stationärer Absorptionsmessungen steht ein UV-VIS-IR-Spektrometer (Varian, Cary 5E, mit CaryWinUV 3.0 Software) zur Verfügung. Bei Messungen in der flüssigen Phase werden Quarzküvetten (Hellmar) mit verschiedenen Schichtlängen (1-10 mm) verwendet. Für die Messungen in überkritischer Phase kann auf eine Hochdruckzelle (MPIBPC, Selbstbau, $P_{\max }=4000$ bar) mit einer optischen Weglänge von $60 \mathrm{~mm}$ und einer Apertur von $10 \mathrm{~mm}$ zurückgegriffen werden. Als Fenstermaterial wird z. B. optisch poliertes Saphir mit einer Dicke von $10 \mathrm{~mm}$ und einem Durchmesser von $20 \mathrm{~mm}$ verwendet. Die Zelle ist über einen umwickelten Heizdraht temperierbar, wobei eine PT-100-basierte Temperatursteuerung (Haake TP24) als Regelung dient. Der zugehörige PT-100-Temperatursensor kann in eine Bohrung im Zellkörper eingebracht werden. Zum Befüllen der Zelle dient ein Anschluss für 1/4" NOVA-Kapillaren. 


\subsection{Verwendete Chemikalien}

Das verwendete Diiodmethan, sowie die benutzten Lösungsmittel und Gase sind mit Reinheits- und Herstellerangaben in Tabelle 3.1 aufgeführt.

\begin{tabular}{|c|c|c|c|}
\hline Substanz & Hersteller & Kurzform & Reinheit \\
\hline Diiodmethan zur Synthese & Merck-Schuchardt & $\mathrm{CH}_{2} \mathrm{I}_{2}$ & $>99 \%$ \\
\hline Methanol Uvasol & Merck & $\mathrm{CH}_{3} \mathrm{OH}$ & min. 99,9\% \\
\hline Acetonitril LiChroSolv & Merck & $\mathrm{CH}_{3} \mathrm{CN}$ & min. 99,9\% \\
\hline Perfluoro- $n$-Hexan & Lancaster & $\mathrm{C}_{6} \mathrm{~F}_{14}$ & $99 \%$ \\
\hline Kohlendioxid & Messer Griesheim & $\mathrm{CO}_{2}$ & 4.5 \\
\hline Trifluormethan & Messer Griesheim & $\mathrm{CHF}_{3}$ & 4.5 \\
\hline Ethan & Messer Griesheim & $\mathrm{C}_{2} \mathrm{H}_{6}$ & 2.5 \\
\hline Xenon & Messer Griesheim & $\mathrm{Xe}$ & 4.0 \\
\hline
\end{tabular}

Tabelle 3.1: Herkunft und Reinheit der verwendeten Chemikalien. 



\section{Kapitel 4}

\section{Experimentelle Ergebnisse}

In diesem Kapitel werden Femtosekunden Pump/Probe-Messungen an Diiodmethan in verschiedenen überkritischen Lösungsmitteln vorgestellt. Insbesondere sind Messungen zur spektralen Dynamik des entstehenden $\mathrm{CH}_{2} \mathrm{I}-\mathrm{I}$ und zur Dichteabhängigkeit der Isomerisierungsgeschwindigkeit und Quantenausbeute an Isomer Gegenstand der Untersuchungen. Die Variation der Dichte und des Lösungsmittels sollen dabei Aufschluss über den Einfluss der Umgebung auf den Mechanismus der Isomerisierung geben.

\subsection{Stationäre Absorptionsmessungen}

Zunächst werden stationäre Absorptionsmessungen vorgestellt, die in dem beschriebenen (siehe Abschnitt 3.7) Cary-Spektrometer durchgeführt wurden. Es sollte festgestellt werden, ob bereits ein Einfluss der Lösungsmitteleigenschaften, wie z. B. Polarität, auf das stationäre Absorptionsspektrum existiert.

\subsubsection{Absorptionsspektren in organischen Lösungsmitteln}

In Abbildung 4.1 findet sich das Absorptionsspektrum in Perfluoro- $n$-Hexan. Im Vergleich dazu sind das Absorptionsspektrum in $n$-Oktan aus [61] und in der reinen Gasphase des $\mathrm{CH}_{2} \mathrm{I}_{2}$ aus [62] dargestellt. Die Spektren wurden zum Vergleich auf das Maximum der ersten Absorptionsbande bei etwa $297 \mathrm{~nm}$ normiert.

Die Diiodmethanspektren in $n$-Oktan und in Perfluoro- $n$-Hexan stimmen mit dem Gasphasenspektrum sehr gut überein. Insbesondere in der Gestalt und in der Position des Maximums der ersten Absorptionsbande sind nur sehr geringe Abweichungen zu erkennen. Im Spektralbereich zwischen 220-270 nm stimmen die Spektren in ihrer Gestalt ebenfalls recht gut überein. Lediglich die relativen Absorptionsmaxima differieren leicht. Die Unterschiede können zum einen auf die unterschiedlichen verwendeten 


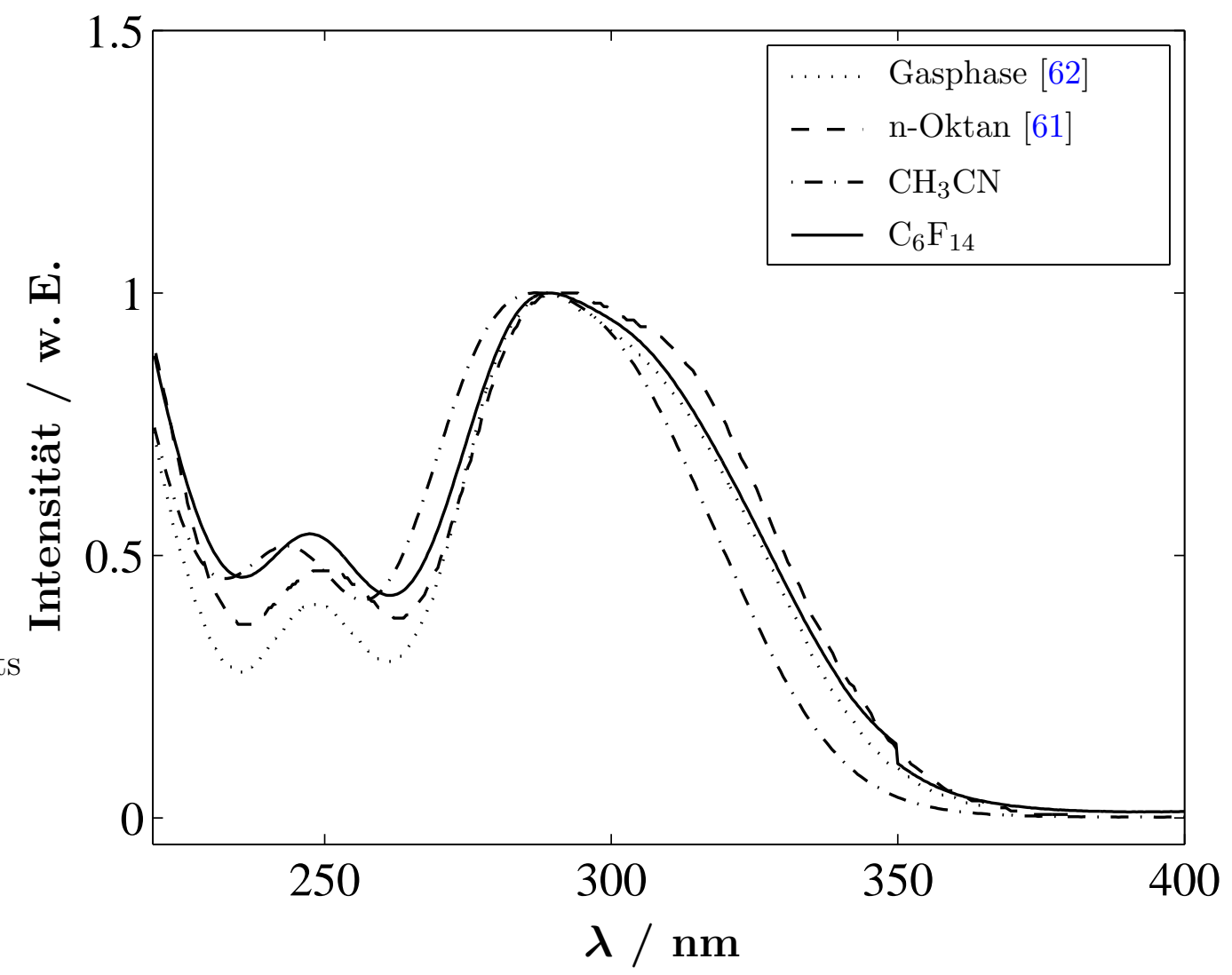

Abbildung 4.1: Stationäre Absorptionsspektren von $\mathrm{CH}_{2} \mathrm{I}_{2}$ in verschiedenen organischen Lösungsmitteln.

Spektrometer und zum anderen auf eine nicht ausreichende Korrektur der Grundabsorption des reinen Lösungsmittels zurückzuführen sein. Aus anderen Arbeiten [139] ist bekannt, dass besonders perfluorierte Alkane einen sehr kleinen Lösungsmitteleinfluss auf die Absorptionsspektren der Solvate haben. Dagegen zeigt sich bei einem polaren Lösungsmittel, wie Acetonitril ein größerer Einfluss. Das gesamte Spektrum ist um etwa $8 \mathrm{~nm}$ in den kürzerwelligeren Spektralbereich verschoben, wobei die Gestalt der ersten Absorptionsbande im wesentlichen erhalten bleibt. Dies deutet auf einen kleinen Einfluss der Polarität des Lösungsmittels auf das stationäre Absorptionsspektrum des Diiodmethans hin [55]. Der Energieabstand des elektronisch angeregten Zustands zum Grundzustand ist in polaren Lösungsmitteln größer als in unpolaren Lösungsmitteln. Daraus lässt sich eine lösungsmittelinduzierte Stabilisierung bzw. Destabilisierung der involvierten elektronischen Zustandes durch polare Lösungsmittel vermuten. 


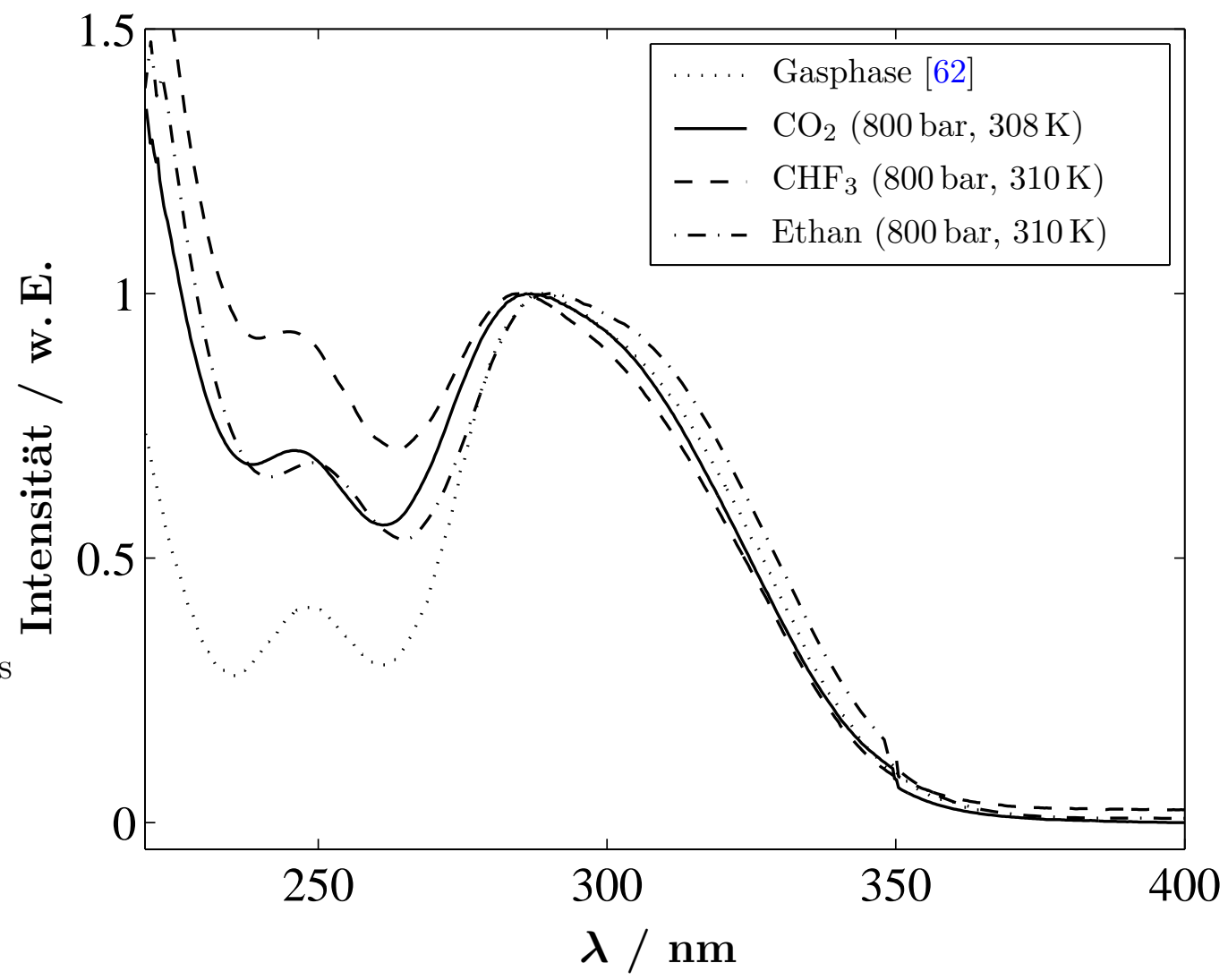

Abbildung 4.2: Stationäre Absorptionsspektren von $\mathrm{CH}_{2} \mathrm{I}_{2}$ in verschiedenen überkritischen Lösungsmitteln.

\subsubsection{Absorptionsspektren in überkritischen Lösungsmitteln}

In Abbildung 4.2 finden sich stationäre Absorptionsspektren des Diiodmethans in verschiedenen überkritischen Lösungsmitteln, die auch für die transienten Absorptionsmessungen verwendet wurden. Die Spektren sind ebenfalls auf das Maximum der ersten Absorptionsbande normiert. Zum Vergleich ist außerdem das Spektrum der reinen Gasphase (punktierte Linie in Abbildung 4.2) dargestellt.

Insbesondere im kürzerwelligerem Spektralbereich zeichnen sich deutliche Abweichungen in der relativen Signalhöhe der Spektren zur Gasphasenmessung ab. Dies kann auf die unzureichende Korrektur des Untergrundes zurückgeführt werden, da lediglich die Absorption der Hochdruckzelle selbst, ohne Füllung, von den gemessenen Spektren subtrahiert wurde. Jedoch lassen sich in dem, für die transienten Messungen relevanten, Spektralbereich um die erste Absorptionsbande nur kleine Abweichungen feststellen. Das Spektrum in $\mathrm{CO}_{2}(308 \mathrm{~K}, 800$ bar $)$ ist nur sehr wenig, das Spektrum in $\mathrm{CHF}_{3}(310 \mathrm{~K}, 800$ bar) wenige Nanometer in den kürzerwelligeren Bereich verscho- 

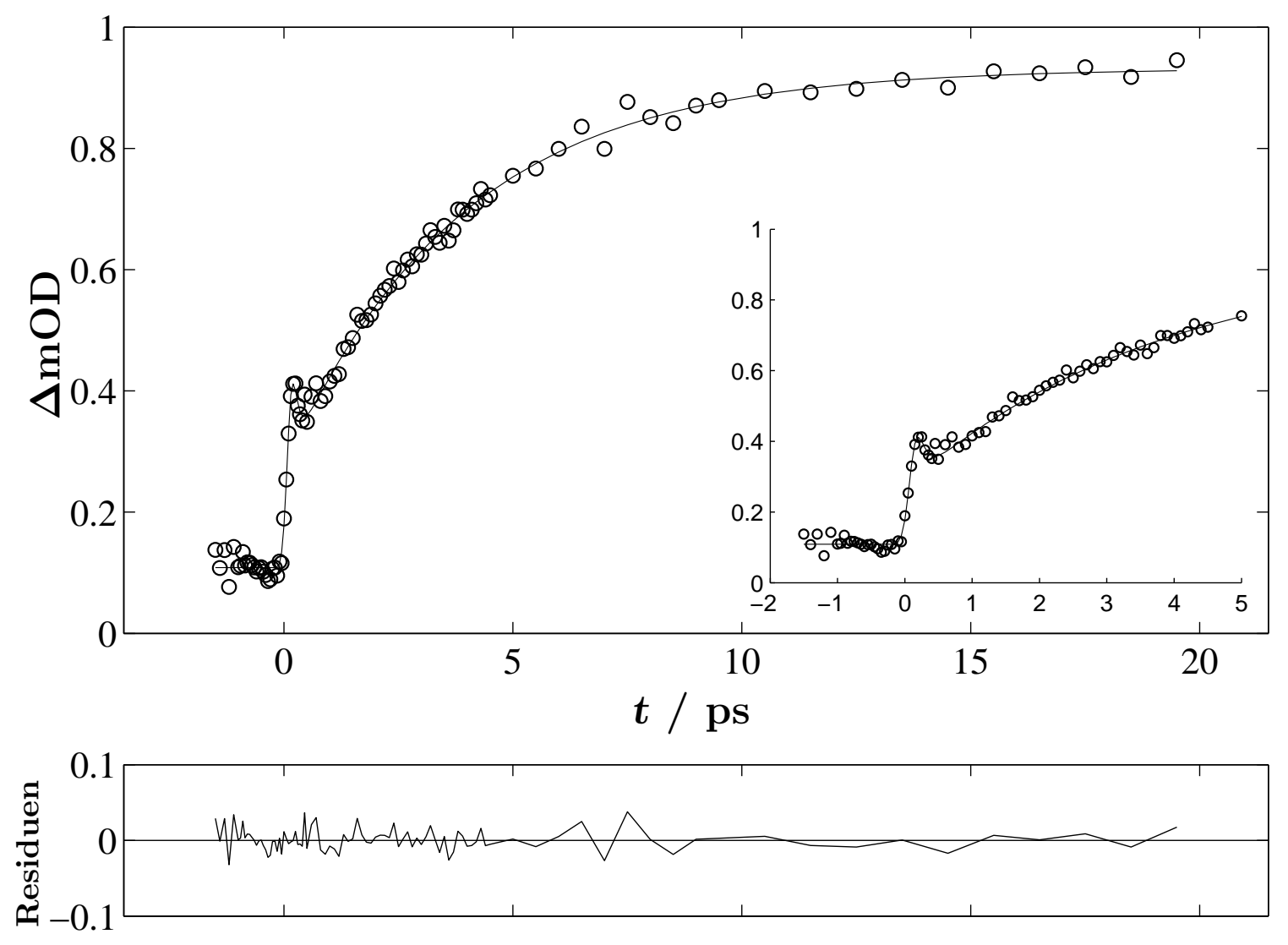

Abbildung 4.3: Transiente Absorption von $\mathrm{CH}_{2} \mathrm{I}_{2}$ in Acetonitril bei $300 \mathrm{~nm}$ Anregung und $610 \mathrm{~nm}$ Abfrage.

ben, wohingegen das Spektrum in Ethan (310 K, 800 bar) um wenige Nanometer zu längeren Wellenlängen verschoben ist. Dies deutet ebenfalls auf eine Einfluss auf die elektronischen Zustände durch polare oder gut polarisierbare Lösungsmittel hin.

\subsection{Zeitaufgelöste Absorptionsmessungen}

\subsubsection{Voruntersuchungen}

Im Folgenden werden zunächst Voruntersuchungen zur Charakterisierung des Messsystems vorgestellt. 


\section{Voruntersuchungen in flüssiger Phase}

In Anlehnung und zum Vergleich zu Åkesson et al. [36], Yosihara et al. [35] und Harris et al. [34] wurden Untersuchungen in flüssiger Phase bei $300 \mathrm{~nm}$ Pump und $630 \mathrm{~nm}$ Probe durchgeführt. Dazu wurden etwa $92 \mathrm{mg}$ Diiodmethan in $100 \mathrm{ml}$ Acetonitril gelöst. Dies entspricht einer Konzentration von 3,4 mmol/l und bei einer Schichtlänge von $0,19 \mu \mathrm{m}$ und einer Anregungswellenlänge von $300 \mathrm{~nm}$ einer optischen Dichte von etwa 2.

Die Messungen (siehe Abbildung 4.3) zeigen einen sehr ähnlichen Verlauf zu den genannten Arbeiten mit folgenden charakteristischen drei Komponenten: einem Anstieg innerhalb der Zeitauflösung, einem schnellen monoexponentiellen Abfall und einem monoexponentiellen ps-Anstieg. Die bisherigen experimentellen Ergebnisse in flüssiger Phase können durch die hier gezeigten Messungen sehr gut reproduziert werden und die Anstiegszeit des Signals mit 3,5 ps liegt quantitativ im gleichen Bereich, wie bei den Messungen von Åkesson et al. (2,8 ps) [36]. Daher sind weitere Untersuchungen auch in überkritischer Phase möglich, deren Ergebnisse direkt mit den Messungen in flüssiger Phase verglichen werden können.

\section{Voruntersuchungen in der Hochdruckzelle}

Zur Bestimmung der Charakteristika des Pump/Probe-Interferometers mit Hochdruckzelle wurde etwas all-trans-Diphenylbutadien (DPB) in die Hochdruckzelle gegeben und in flüssigem $\mathrm{CO}_{2}(295 \mathrm{~K})$ gelöst. Die in Abbildung 4.4 gezeigte Abklingkurve wurde bei $300 \mathrm{~nm}$ Pump und $630 \mathrm{~nm}$ Probe sowie einem Druck von 120 bar erhalten. Die Anpassung der Signale in DPB ergab im Mittel eine Kreuzkorrelationsbreite der Pulse und damit eine Zeitauflösung des Experiments von etwa 200 fs. Dies ist möglich weil die transiente Absorption von DPB instantan einsetzt [131]. 

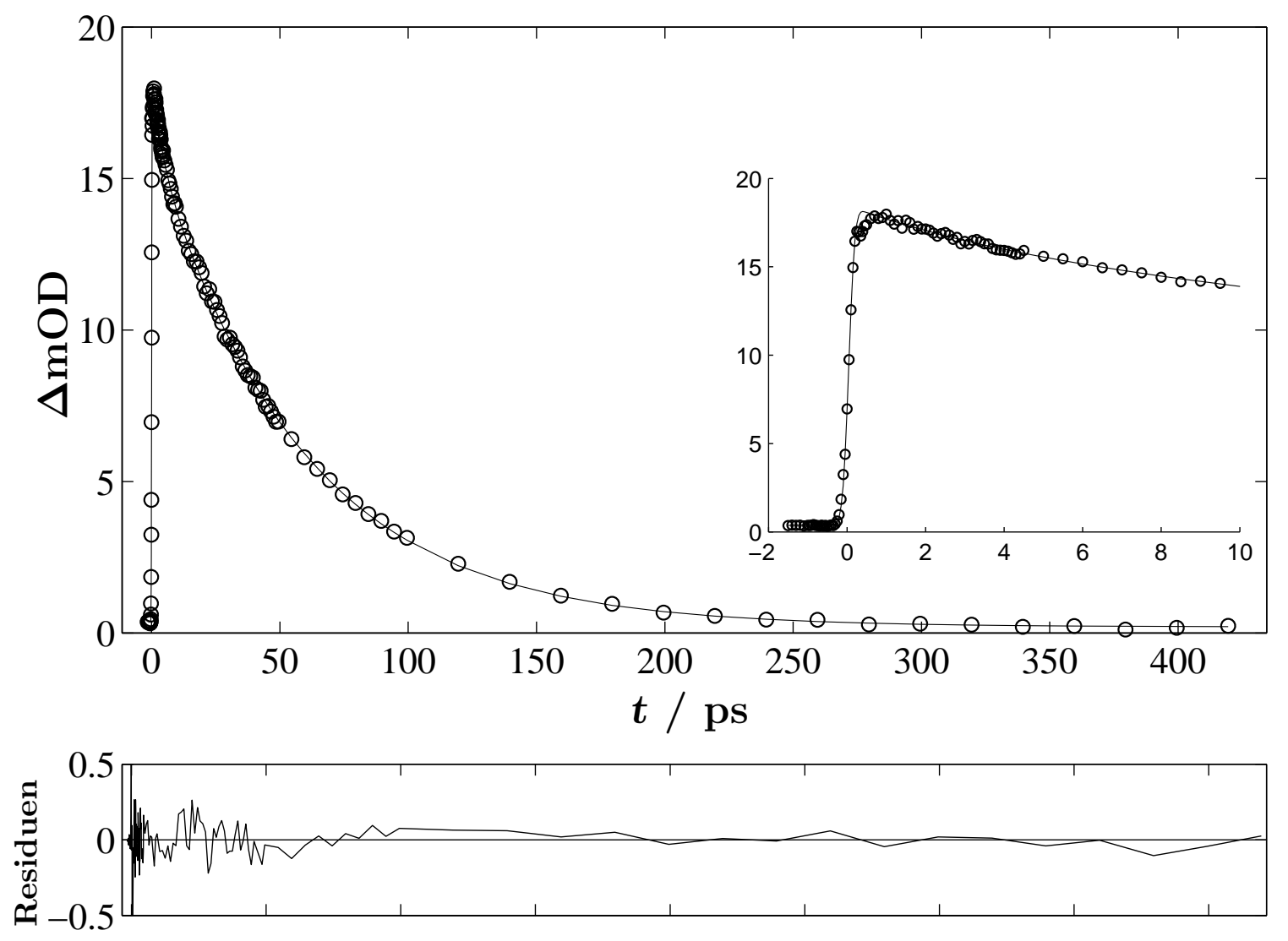

Abbildung 4.4: Erstes Signal in der Hochdruckzelle: DPB in $\mathrm{CO}_{2}$ (120 bar, $308 \mathrm{~K}$ bei $300 \mathrm{~nm}$ Anregung und $630 \mathrm{~nm}$ Abfrage.

\subsubsection{Zeitaufgelöste Messungen in überkritischem $\mathrm{CO}_{2}$}

Für die ersten Messungen in überkritischen Fluiden wurde Kohlendioxid $\mathrm{CO}_{2}$ als Lösungsmittel ausgewählt. $\mathrm{CO}_{2}$ ist nicht giftig, leicht handhabbar und sowohl die kritische Temperatur mit 304,2 K als auch der kritische Druck von 73,8 bar [140] liegen in experimentell gut erreichbaren Regionen. Außerdem besitzt es kein permanentes Dipolmoment, sondern nur ein Quadrupolmoment. Es verfügt in der überkritischen Phase über gute Lösungsmitteleigenschaften.

Für die, in den folgenenden Abschnitten gezeigten, zeitaufgelösten Messungen wurden meist $3 \mu \mathrm{l}$ des Diiodmethans mit einer Mikroliterspritze vor Beginn der Messreihe in die Hochdruckzelle injiziert. Dies entspricht bei einer Anregungswellenlänge von $300 \mathrm{~nm}$ gerade einer optischen Dichte von 2-3 für den Pumpstrahl. Unter der Annahme, das sämtliches Diiodmethan in Lösung vorliegt bedeutet dies eine Konzentration von ca. $15 \mathrm{mmol} \cdot \mathrm{l}^{-1}$. Bei dieser Konzentration und einer Pumpenergie bei $300 \mathrm{~nm}$ von max. 50 nJ pro Puls, ist unter der Annahme, dass jedes Photon eine Reaktion zu den 

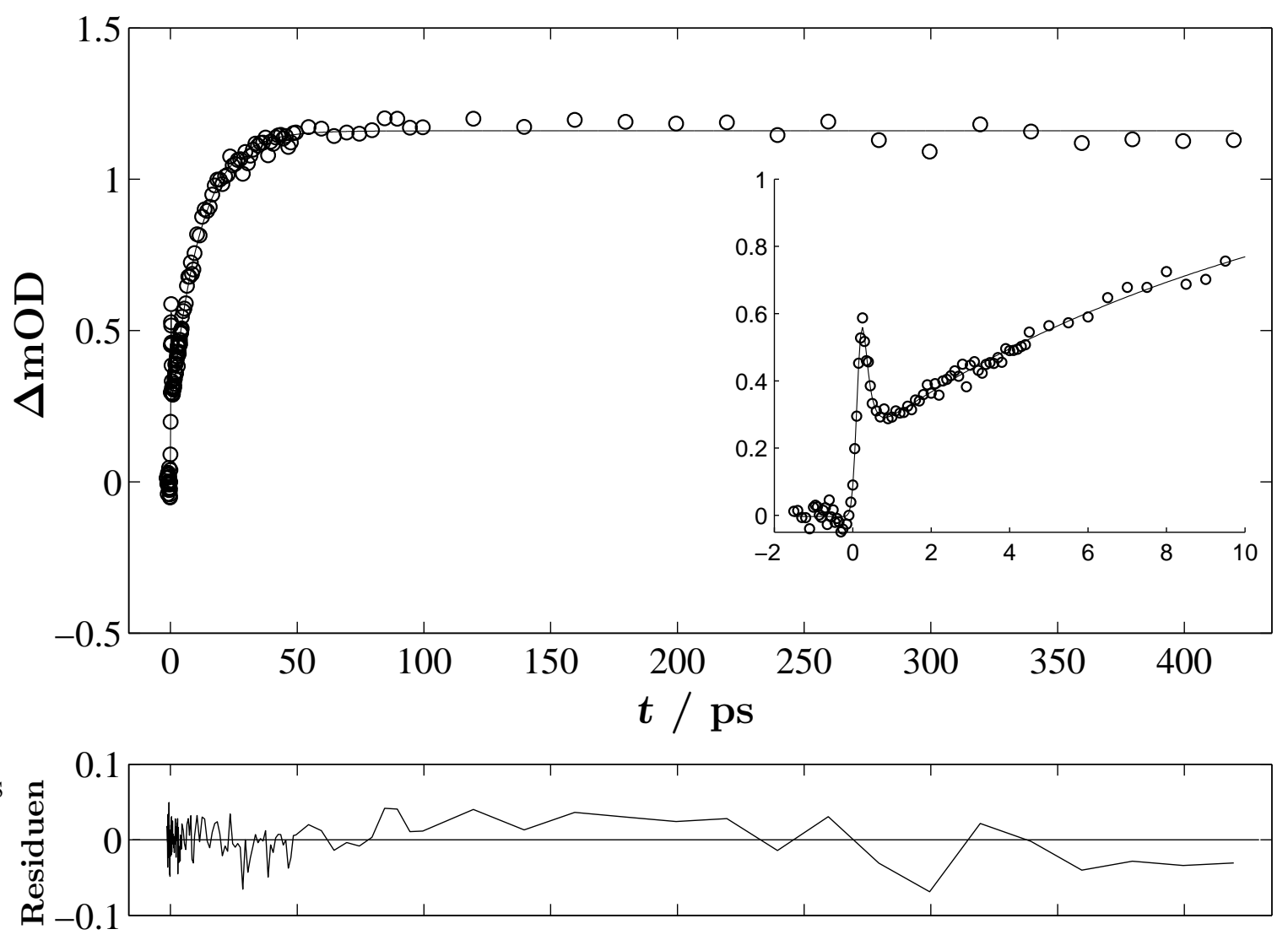

Abbildung 4.5: Typisches Signal in überkritischem $\mathrm{CO}_{2}(399 \mathrm{bar}, 308 \mathrm{~K})$ bei $304 \mathrm{~nm}$ Anregung und $405 \mathrm{~nm}$ Abfrage.

Photoprodukten auslöst, innerhalb einer Stunde Bestrahlung mit Pumplicht nur jedes zweihunderste Molekül zerfallen. Diese Abschätzung und die Tatsache, dass keinerlei Ausbleichen des Signals beim Hin- und Zurückfahren der Verschiebebühne zu verzeichnen war, zeigt, dass keine Konzentrationsabhängigkeit der Signale vorliegt. Ebenfalls durchgeführte Messungen mit verschiedenen Konzentrationen an $\mathrm{CH}_{2} \mathrm{I}_{2}$ im Bereich von 5-30 mmol.1 ${ }^{-1}$ zeigten keine Änderungen der Signale. Ab einem Druck von etwa 100 bar hatte sich das Diiodmethan in der Zelle homogen gelöst und der Pumpstrahl wurde nahezu vollständig absorbiert. Eine typische zeitaufgelöste, transiente Messung in überkritischem $\mathrm{CO}_{2}$ zeigt Abbildung 4.5.

\section{Spektrale Entwicklung}

Zunächst sollte die spektrale Abhängigkeit des Signals von der Probewellenlänge bei einem festem Druck untersucht werden. Dazu wurden zu verschiedenen Probewellen- 


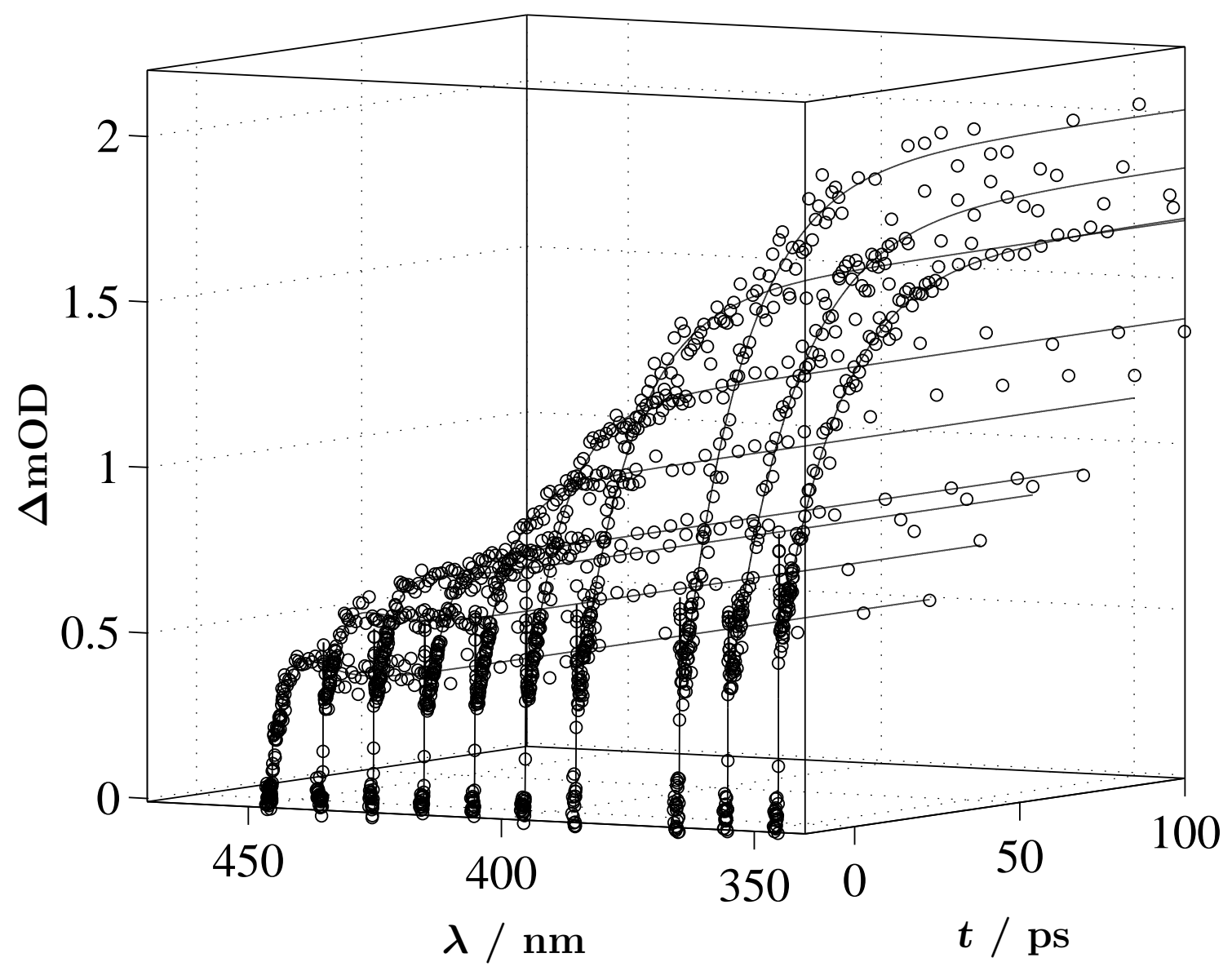

Abbildung 4.6: Transiente Messungen in überkritischem $\mathrm{CO}_{2}$ bei verschiedenen Probewellenlängen.

längen transiente Absorptionsignale aufgenommen (siehe Abbildung 4.6) [108, 141]. Bei Wechsel der Probewellenlänge wurde der räumliche Überlapp zwischen Pumpund Probestrahl in der Zelle gegebenenfalls leicht nachoptimiert, da sich die räumliche Lage des Probestrahls auf Grund der Winkelabhängigkeit der nichtlinearen, optischen Prozesse im TOPAS leicht ändern kann.

Die so erhaltenen Kurven wurden mit dem Auswertprogramm angepasst und so der genaue Zeitnullpunkt $t_{0}$ und der Gesamtoffset $I_{1}$ ermittelt. Die Absorptions-Zeitprofile werden dahingehend korrigiert, dass der angepasste Gesamtoffset $I_{1}$ von den $\triangle O D$ Werten und der angepasste Zeitnullpunkt $t_{0}$ von den Zeitwerten abgezogen wird. Anschließend werden zu einer bestimmten Verzögerungszeit aus jeder einzelnen Absorptionskurve Werte entnommen und in Abhängigkeit der Probewellenlänge dargestellt. Dieses Verfahren führt zu einem rekonstruierten, zeitabhängigen Spektrum, wie es in Abbildung 4.7 dargestellt ist. 


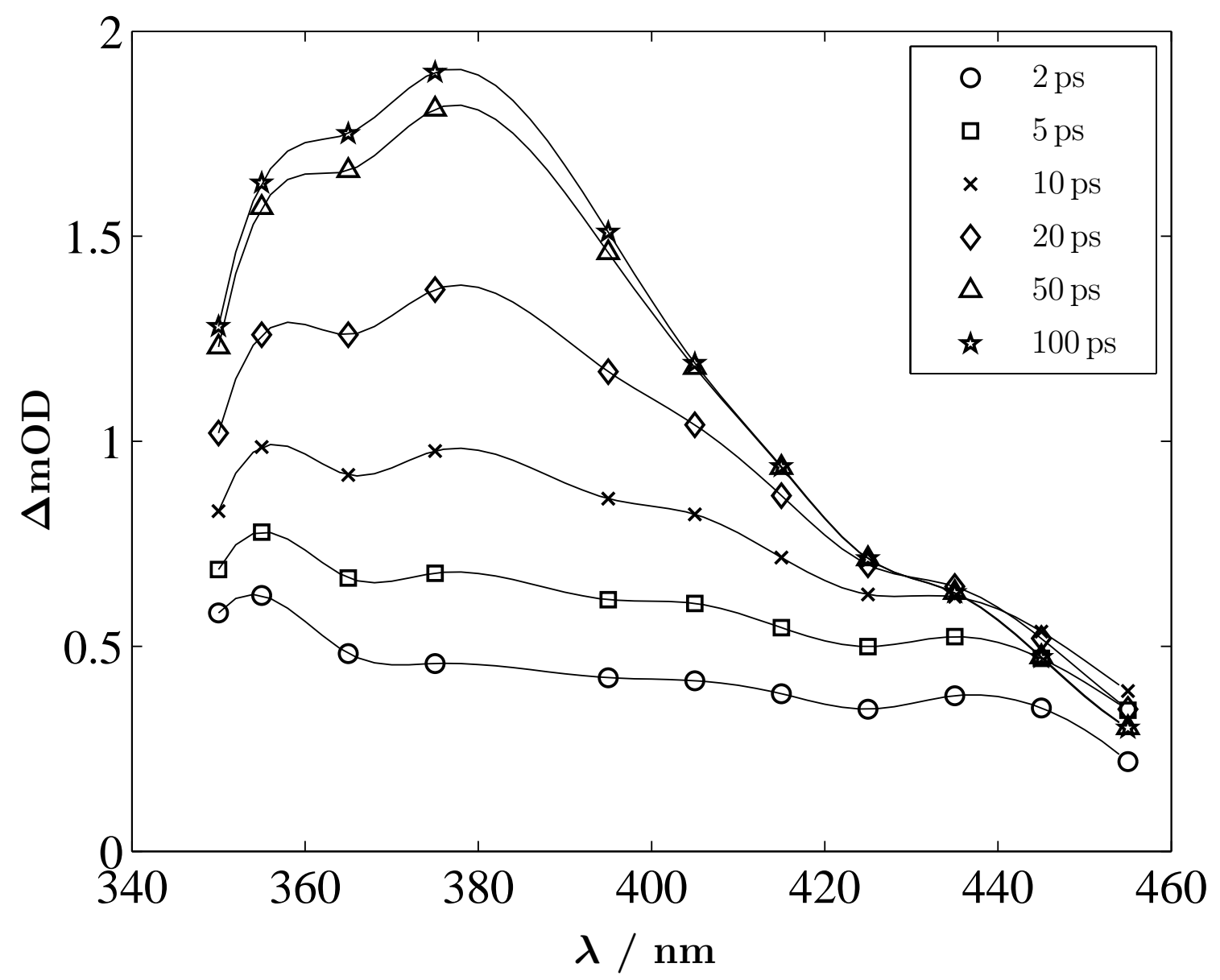

Abbildung 4.7: Transientes Absorptionsspektrum in überkritischem $\mathrm{CO}_{2}(308 \mathrm{~K}$, 400 bar) bei $300 \mathrm{~nm}$ Anregung zu langen Zeiten.

Das transiente Absorptionsspektrum zu langen Zeiten (siehe Abbildung 4.7) stimmt in seiner zeitlichen Entwicklung und Gestalt gut mit dem von Åkesson et al. [36] gemessenen Spektrum überein. Eine breite Bande von 350-430 nm, zentriert bei $370 \mathrm{~nm}$, wächst mit der Zeit an. Diese Bande kann gemäß früheren Arbeiten [33,36] dem entstehenden $\mathrm{CH}_{2} \mathrm{I}-\mathrm{I}$-Isomer zugeordnet werden. Unter der Annahme, dass nach einer Zeit von 2 ps nach der Anregung im Wesentlichen nur noch die spektrale Dynamik des absorbierenden Isomers im Spektrum sichtbar ist, kann das Spektrum auf die Population des Isomers normiert werden. Dies geschieht durch Normieren der Spektren zu verschiedenen Zeiten auf die Fläche unter dem Spektrum, welches jedoch in diesem Fall fehlerbehaftet sein kann, da nicht der gesamte Spektralbereich experimentell zugänglich war. Ein so erhaltenes populationsnormiertes Spektrum ist in Abbildung 4.8 dargestellt. 


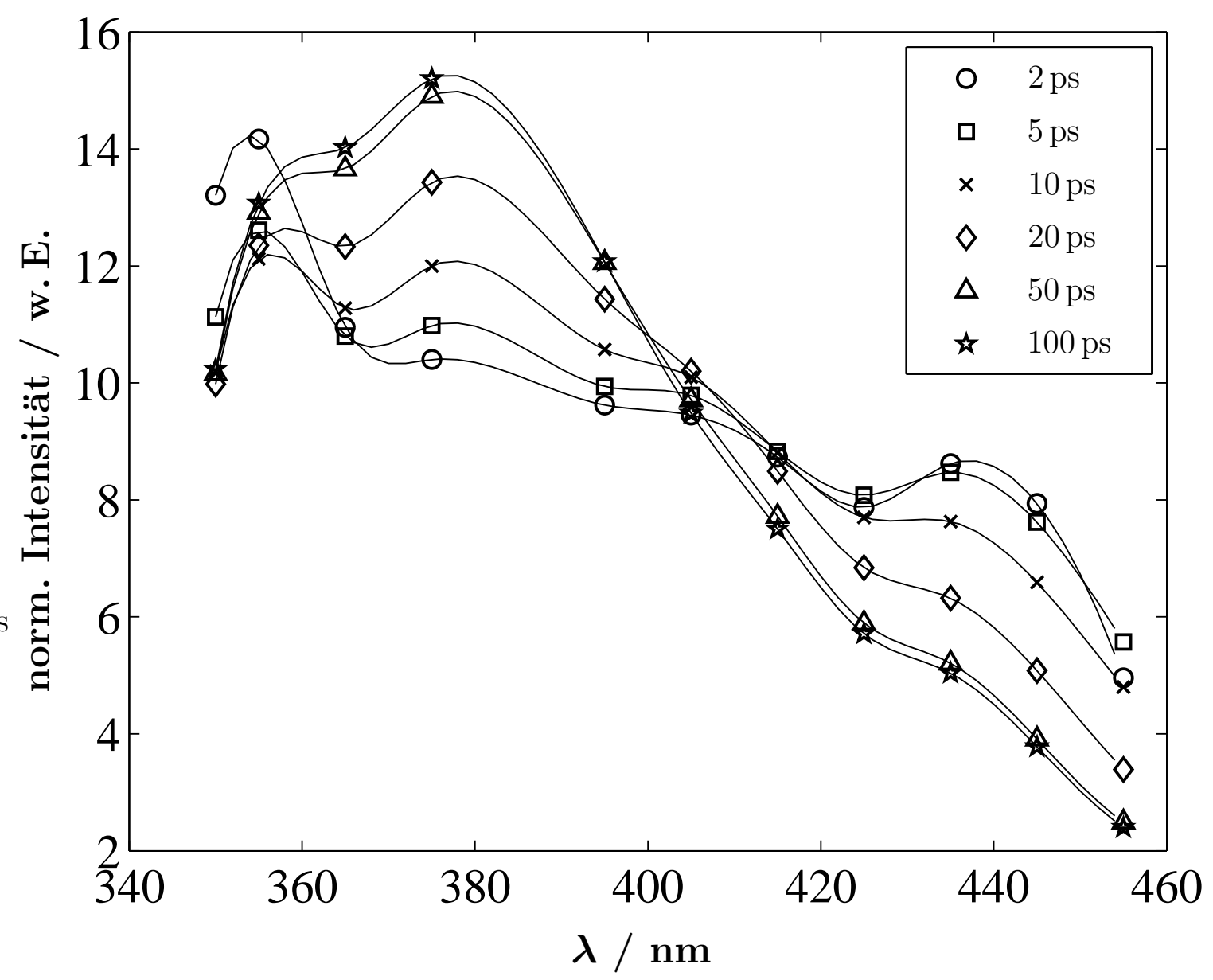

Abbildung 4.8: Auf Fläche normiertes, transientes Absorptionsspektrum in überkritischem $\mathrm{CO}_{2}(308 \mathrm{~K}, 400$ bar) bei $304 \mathrm{~nm}$ Anregung zu langen Zeiten.

Das Spektrum zu kurzen Zeiten bis 2 ps nach der Anregung (siehe Abbildung 4.9) zeigt hauptsächlich eine relativ schmale Bande bei $355 \mathrm{~nm}$ und eine niedrigere sehr breite Bande bei etwa $370 \mathrm{~nm}$. Beide Banden fallen zunächst mit der Zeit ab und und haben, bei Vernachlässigung des Spektrums bei 0 ps, bei etwa 1 bzw. 0,5 ps ein Minimum und wachsen anschließend wieder leicht an. Die beobachtete zeitliche Entwicklung des transienten Spektrums in dem untersuchten Spektralbereich ist qualitativ sehr ähnlich zu dem von Åkesson et al. veröffentlichten Spektrum [36]. Unter erneuter Annahme, dass in diesem Zeitbereich im Wesentlichen nur eine Population zur spektralen Dynamik beiträgt, wurde auch hier eine Normierung vorgenommen (siehe Abbildung 4.10).

Die normierten Kurzzeitspektren zeigen ein Anwachsen und Abfallen innerhalb der ersten 1,5 ps im Bereich der Banden um $355 \mathrm{~nm}$ und $370 \mathrm{~nm}$. Im übrigen Spektralbereich ist nahezu keine spektrale Dynamik sichtbar. 


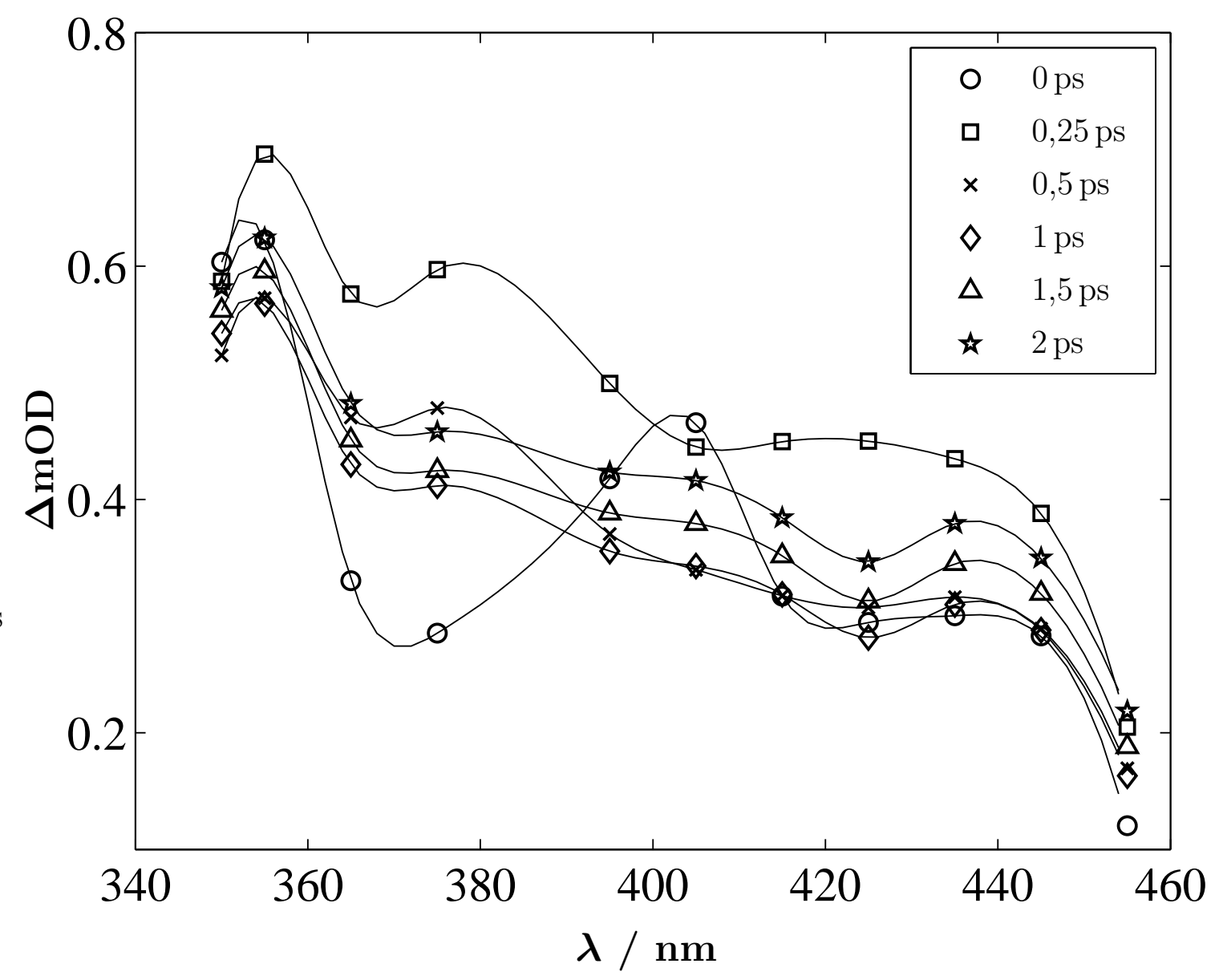

Abbildung 4.9: Transientes Absorptionsspektrum in überkritischem $\mathrm{CO}_{2}\left(35^{\circ} \mathrm{C}\right.$, 400 bar) bei $300 \mathrm{~nm}$ Anregung zu kurzen Zeiten.

Das normierte Langzeitspektrum nach 2 ps (siehe Abbildung 4.8) zeigt ein zeitliches Anwachsen der Bande bei $370 \mathrm{~nm}$ und ein Abfallen im Spektralbereich von 420-450 nm. Der Hauptunterschied zwischen dem normierten Spektrum zu kurzen und langen Verzögerungszeiten besteht darin, dass die Bande bei $370 \mathrm{~nm}$ im Kurzzeitspektrum nur sehr schwach ausgeprägt ist, während sie im Langzeitspektrum stark anwächst. Die im Kurzzeitspektrum stark ausgeprägte schmale Bande bei $355 \mathrm{~nm}$ ist im Langzeitspektrum nur noch als Schulter zu erkennen. Ferner ist im Langzeitspektrum im Bereich um etwa $405 \mathrm{~nm}$ ein isosbestischer Bereich zu erkennen, in dem offensichtlich keine oder nur geringe spektrale Dynamik stattfindet. Dieses spektrale Verhalten mit einem Ansteigen im Bereich einer Absorptionsbande und einem Abfallen an dessen Flanke, sowie das Vorhandensein einer isosbestischen Region deutet auf eine spektrale Verschmälerung der Bande und damit auf eine abkühlende Spezies hin [142, 143, 144, 145, 146]. Im Einklang mit den Spektren von Åkesson et al. [36] kann dies dem Abkühlen des 


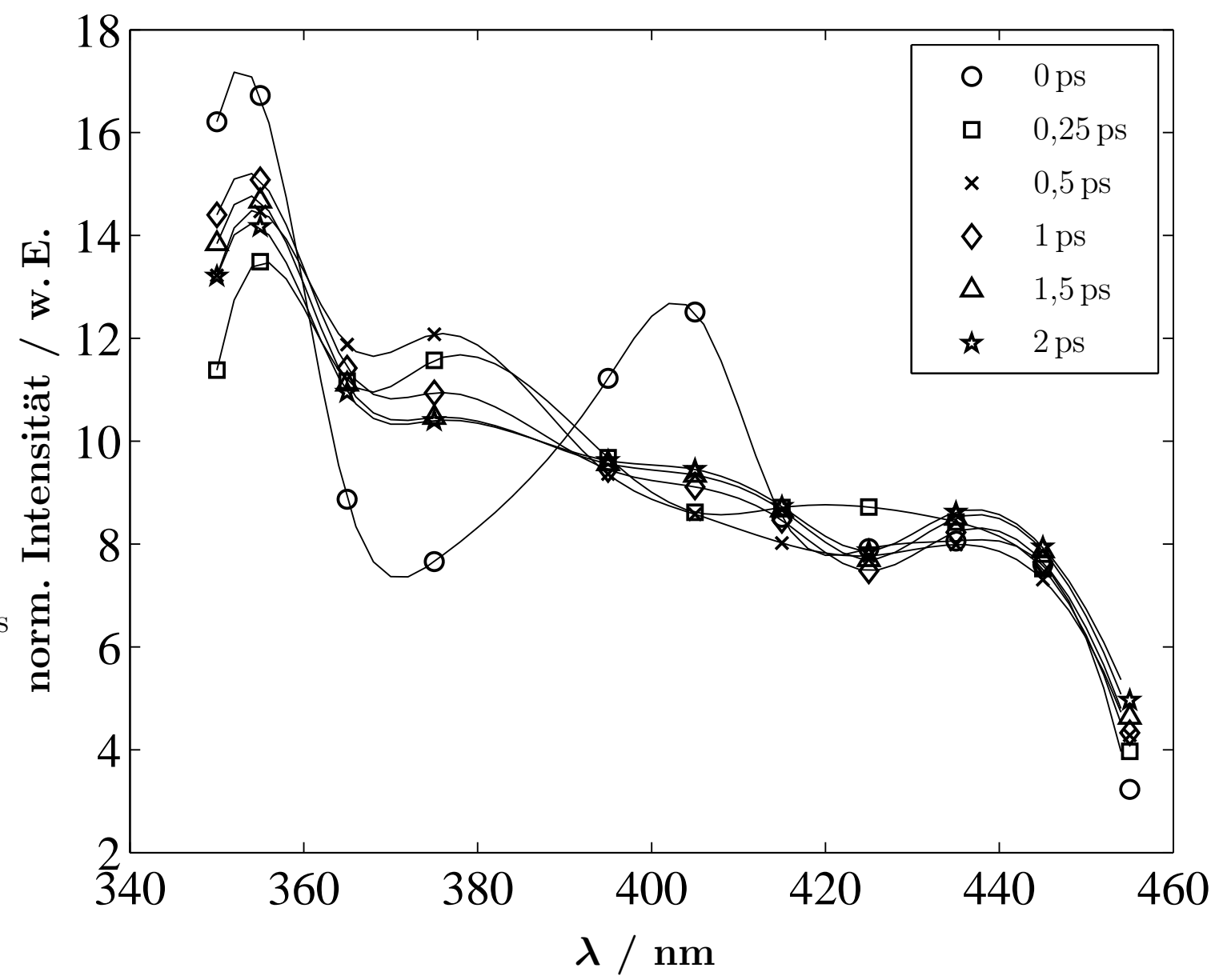

Abbildung 4.10: Auf Fläche normiertes, transientes Absorptionsspektrum in überkritischem $\mathrm{CO}_{2}(308 \mathrm{~K}, 400$ bar) bei $300 \mathrm{~nm}$ Anregung zu kurzen Zeiten.

schwingungsheiß gebildeten Isomers zugeordnet werden. Um die Einflüsse dieser spektralen Dynamik zu vernachlässigen werden die im Folgenden beschriebenen kinetischen Untersuchungen zumeist in der isosbestischen Region durchgeführt.

\section{Dichteabhängigkeiten}

Dichteabhängigkeit der Absorptions-Zeit-Profile Zur Bestimmung der Dichteabhängigkeit der transienten Absorption wurden Messungen bei verschiedenen Drücken aufgenommen (siehe auch Anhang A.2.1). Die Anregungs- und Abfragewellenlänge, sowie die Temperatur der Zelle, waren dabei konstant eingestellt. 


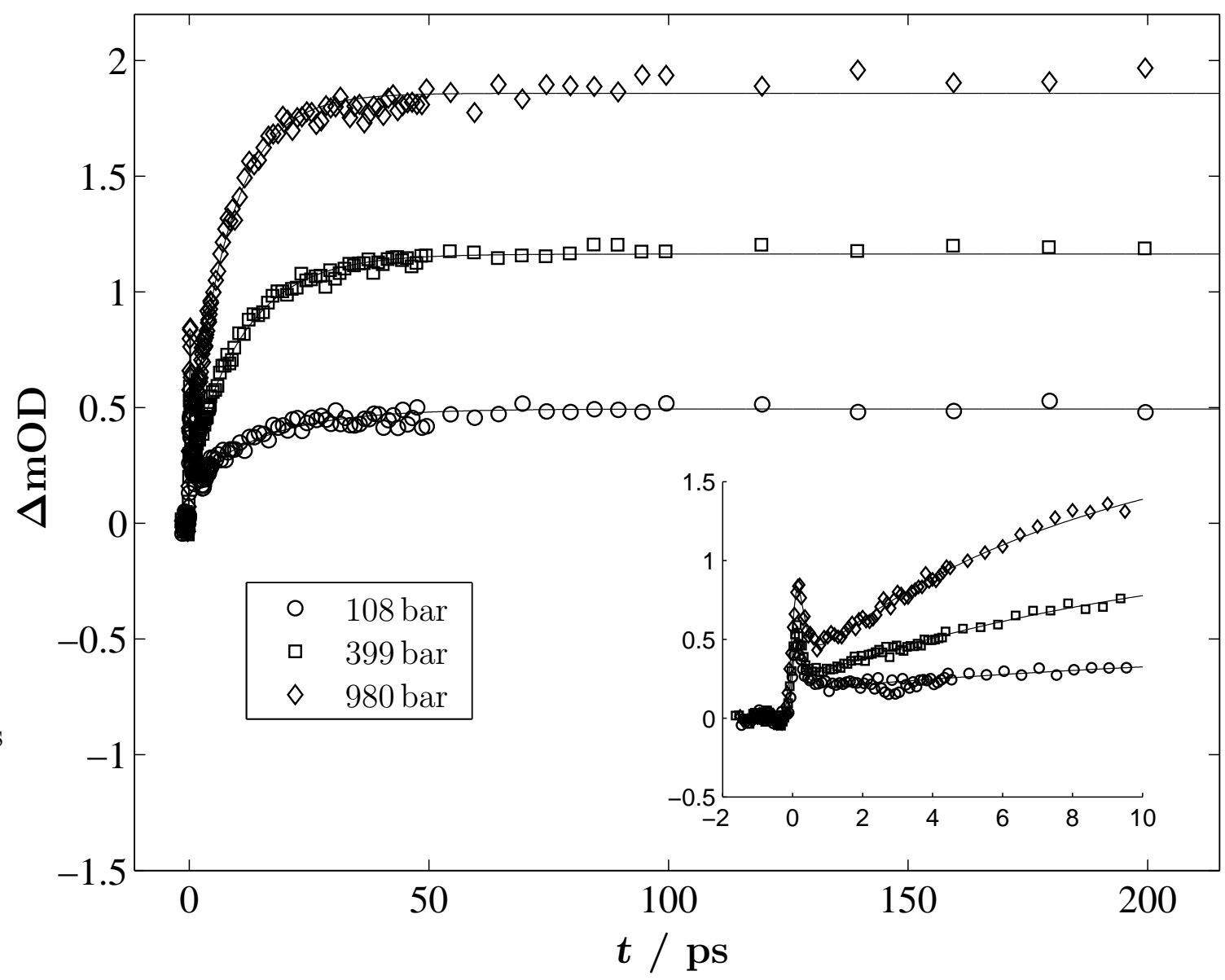

Abbildung 4.11: Druckabhängige Messungen in überkritischem $\mathrm{CO}_{2}(308 \mathrm{~K})$ bei $304 \mathrm{~nm}$ Anregung und $405 \mathrm{~nm}$ Abfrage.

Anhand der in Abbildung 4.11 dargestellten Messungen kann direkt qualitativ eine Druckabhängigkeit der transienten Signale festgestellt werden. Die Zeitkonstante $\tau_{\text {Anstieg }}$ des Anstiegs wird mit zunehmenden Druck kleiner. Die zugehörige Amplitude $A_{\text {Anstieg}}$, die Endamplitude abzüglich des Gesamtoffstets $I_{1}$, steigt mit zunehmenden Druck an (siehe auch Abbildung 4.12).

Aus der Auftragung der angepassten Zeitkonstanten $\tau_{\text {Anstieg }}$ (siehe Abbildung 4.12) gegen den Druck wird ein nahezu exponentieller Verlauf der mit dem Druck schneller werdenden Anstiegszeit sichtbar. Das Ansteigen der zugehörigen Amplitude hängt nahezu exponentiell mit dem Druck zusammen. Bei der Auftragung der Amplitude wird eine größere Streuung der Werte sichtbar, da die Absoluthöhe der Amplitude justageabhängig und somit stärker fehlerbehaftet ist.

Um Aussagen über die Dichteabhängigkeit der kinetischen und dynamischen Prozesse treffen zu können, ist zunächst die Umrechnung der Druckskala in eine Dichteskala not- 


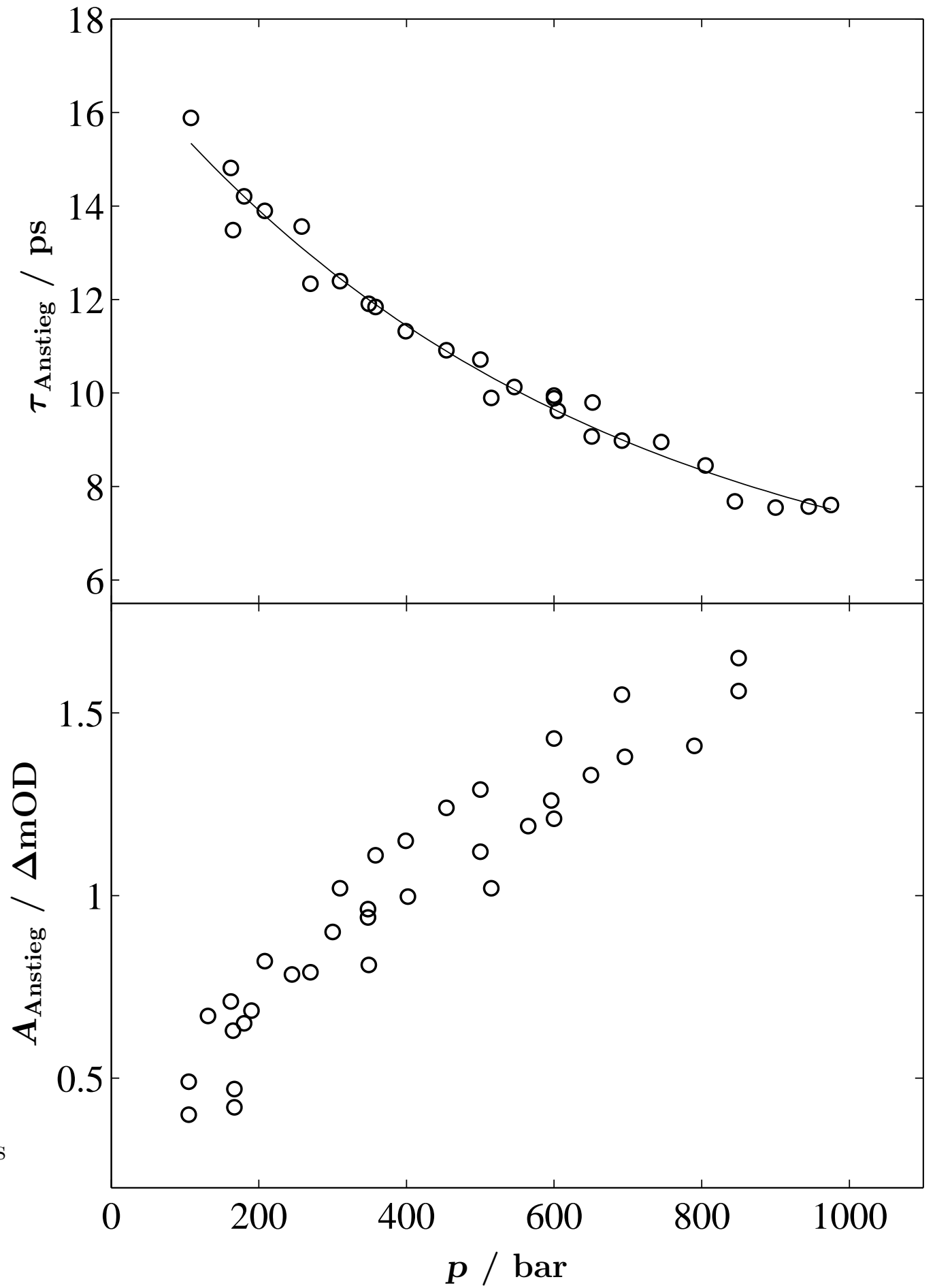

Abbildung 4.12: Druckabhängigkeit der Zeitkonstanten des Anstiegs $\tau_{\text {Anstieg }}$ und der Amplitude $A_{\text {Anstieg }}$ in überkritischem $\mathrm{CO}_{2}(308 \mathrm{~K})$ bei $304 \mathrm{~nm}$ Anregung und $405 \mathrm{~nm}$ Abfrage. 
wendig. Dazu wurden Druck-/Dichtedaten aus dem NIST Chemistry WebBook [140] verwendet (für eine genauere Beschreibung siehe Abbildung A.1 in Anhang A.3.1).

Nach dieser Umrechnung wird der in Abbildung 4.13 gezeigte Zusammenhang der Zeitkonstanten $\tau_{\text {Anstieg}}$, sowie deren zugehörige Amplitude $A_{\text {Anstieg}}$, mit der Dichte erhalten.

Den Auftragungen ist zu entnehmen, dass die Amplitude zwar linear mit der Dichte skaliert, jedoch die Zeitkonstante nicht linear mit der Dichte abnimmt.

Für eine kinetische Beschreibung der Vorgänge bei der Isomerisierung des Diiodmethans werden Geschwindigkeitskonstanten herangezogen. Daher wird in Abbildung 4.14 die Geschwindigkeitskonstante $k_{\text {Anstieg }}=1 / \tau_{\text {Anstieg }}$ gegen den Druck, bzw. gegen die Dichte aufgetragen. Für diese Auftragung wird ein linearer Zusammenhang der Geschwindigkeitskonstanten $k_{\text {Anstieg }}$ mit dem Druck und ein nahezu exponentieller Zusammenhang mit der Dichte erhalten.

In Abbildung 4.15 ist ein Vergleich der Dichteabhängigkeit der Geschwindigkeitskonstanten $k_{\text {Anstieg }}$ bei verschiedenen Wellenlängen dargestellt. Dazu wurde jeweils eine druckabhängige Messreihe im Maximum der Absorptionsbande, bei $370 \mathrm{~nm}$ und eine Messreihe im längerwelligeren Bereich relativ zur isosbestischen Region bei $435 \mathrm{~nm}$ durchgeführt. Die Anpassungen bei $435 \mathrm{~nm}$ mussten mit einer anderen Modellfunktion angepasst werden, da das Signal zu langen Zeiten wieder leicht abfällt (Monoexponentieller Anstieg + Biexponentieller Abfall, siehe Anhang A.1). Die zugehörigen Amplituden wurden jeweils, zur Vergleichbarkeit, auf das erste Maximum des AbsorptionsZeitprofils normiert. In allen drei Fällen wächst der Verlauf der Geschwindigkeitskonstanten exponentiell mit der Dichte, jedoch sind die Absolutwerte nicht gleich. Die absoluten Werte für $k_{\text {Anstieg }}$ liegen für $370 \mathrm{~nm}$ deutlich unter und für $435 \mathrm{~nm}$ deutlich über den Werten in der isosbestischen Region bei $405 \mathrm{~nm}$. Dieses Verhalten ist auf die spektrale Dynamik, die dem Abkühlen der schwingungsheißen Population zugeordnet wird, zurückzuführen. Die zugehörigen normierten Amplituden $A_{\text {Anstieg,norm }}$ werden mit zunehmender Wellenlänge kleiner.

Falls das transiente Absorptionsverhalten nach einer Verzögerungszeit von 100 ps nur von einer Photoproduktspezies, nämlich dem $\mathrm{CH}_{2} \mathrm{I}$-I-Isomer, herrührt, wie von Åkesson et al. postuliert, sollte die Form der Dichteabhängigkeit der normierten Endamplituden für verschiedene spektrale Positionen innerhalb der Absorptionsbande gleich sein. Dazu sind in Abbildung 4.16 die aufeinander skalierten, relativen Amplituden $A_{\text {Anstieg,norm,rel }}$ bei drei verschiedenen Wellenlängen aufgetragen. Die normierten Amplituden wurden dafür um den Faktor 1 bei $405 \mathrm{~nm}$, 0,61 bei $370 \mathrm{~nm}$ und 1,75 bei $435 \mathrm{~nm}$ skaliert. Dabei ist zu erkennen, das innerhalb der experimentellen Unsicherheit, die relativen, normierten Amplituden bei verschiedenen Probewellen eine identische Dichteabhängigkeit zeigen. Überraschender ist die Tatsache, dass auch die re- 


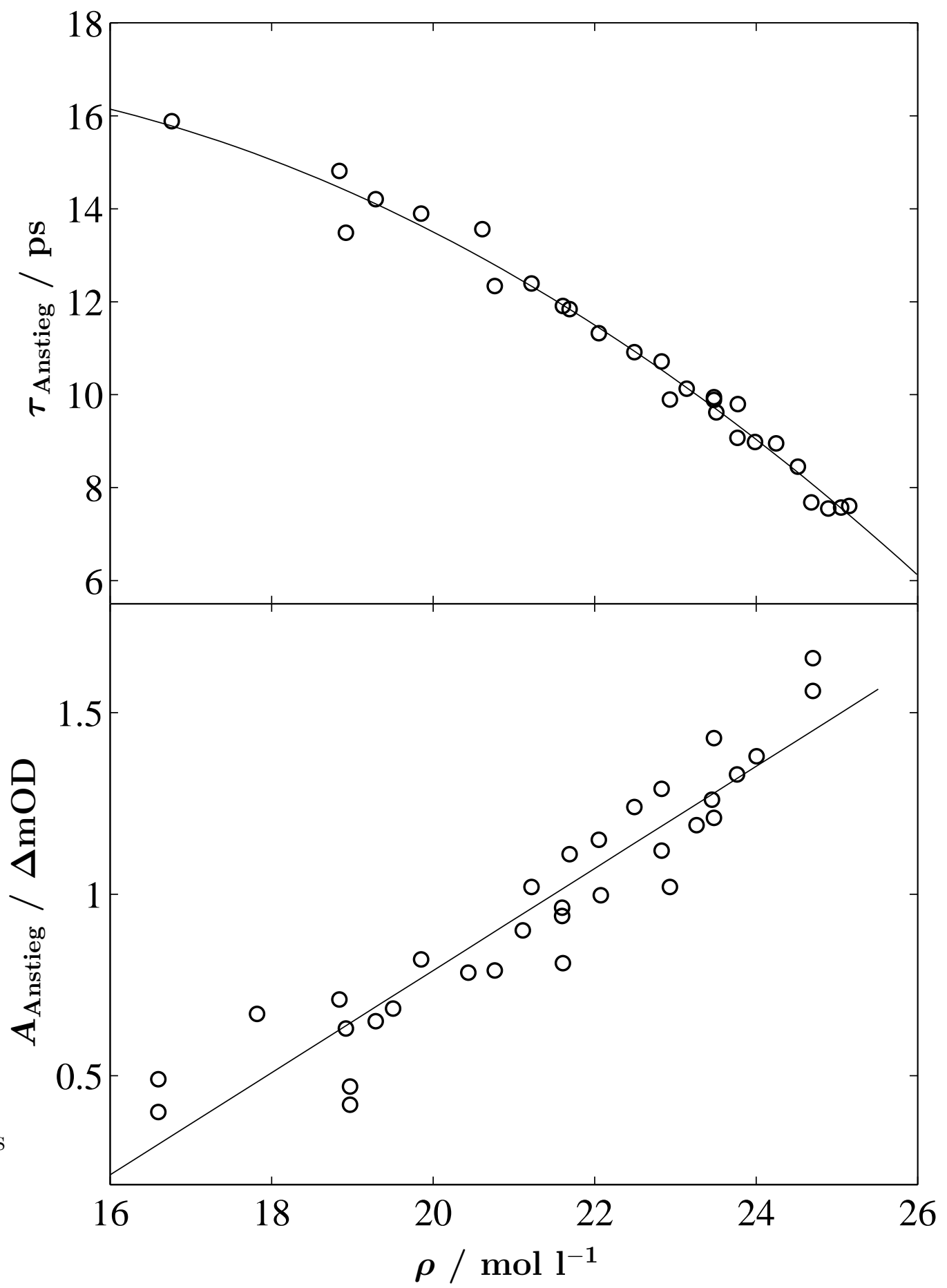

Abbildung 4.13: Dichteabhängigkeit der Zeitkonstanten des Anstiegs $\tau_{\text {Anstieg }}$ und der relativen Amplitude $A_{\text {Anstieg }}$ in überkritischem $\mathrm{CO}_{2}(308 \mathrm{~K})$ bei $304 \mathrm{~nm}$ Anregung und $405 \mathrm{~nm}$ Abfrage. 

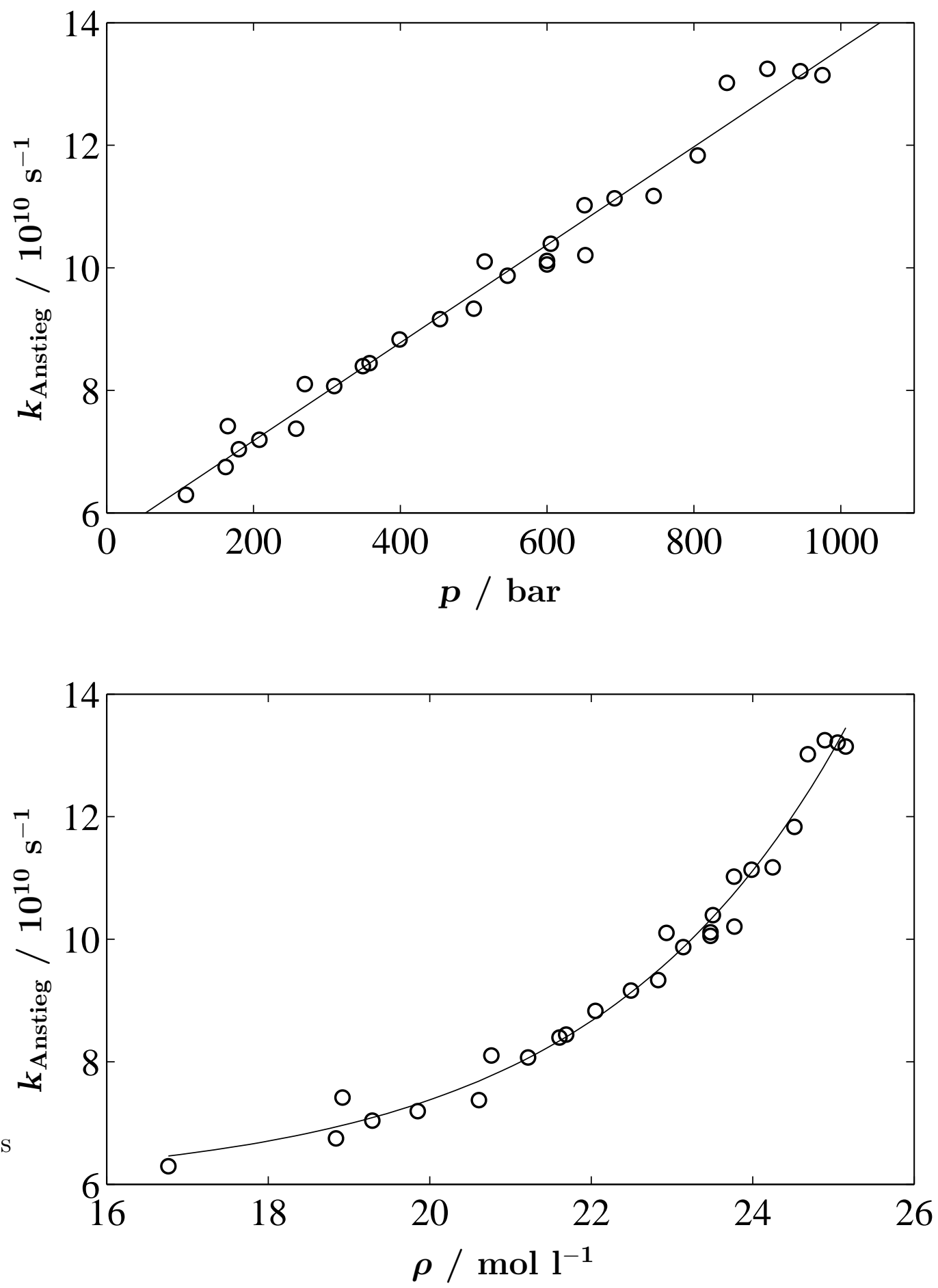

Abbildung 4.14: Druck- bzw. Dichteabhängigkeit der Geschwindigkeitskonstanten des Anstiegs $k_{\text {Anstieg }}$ in überkritischem $\mathrm{CO}_{2}(308 \mathrm{~K})$ bei $304 \mathrm{~nm}$ Anregung und $405 \mathrm{~nm}$ Abfrage. 


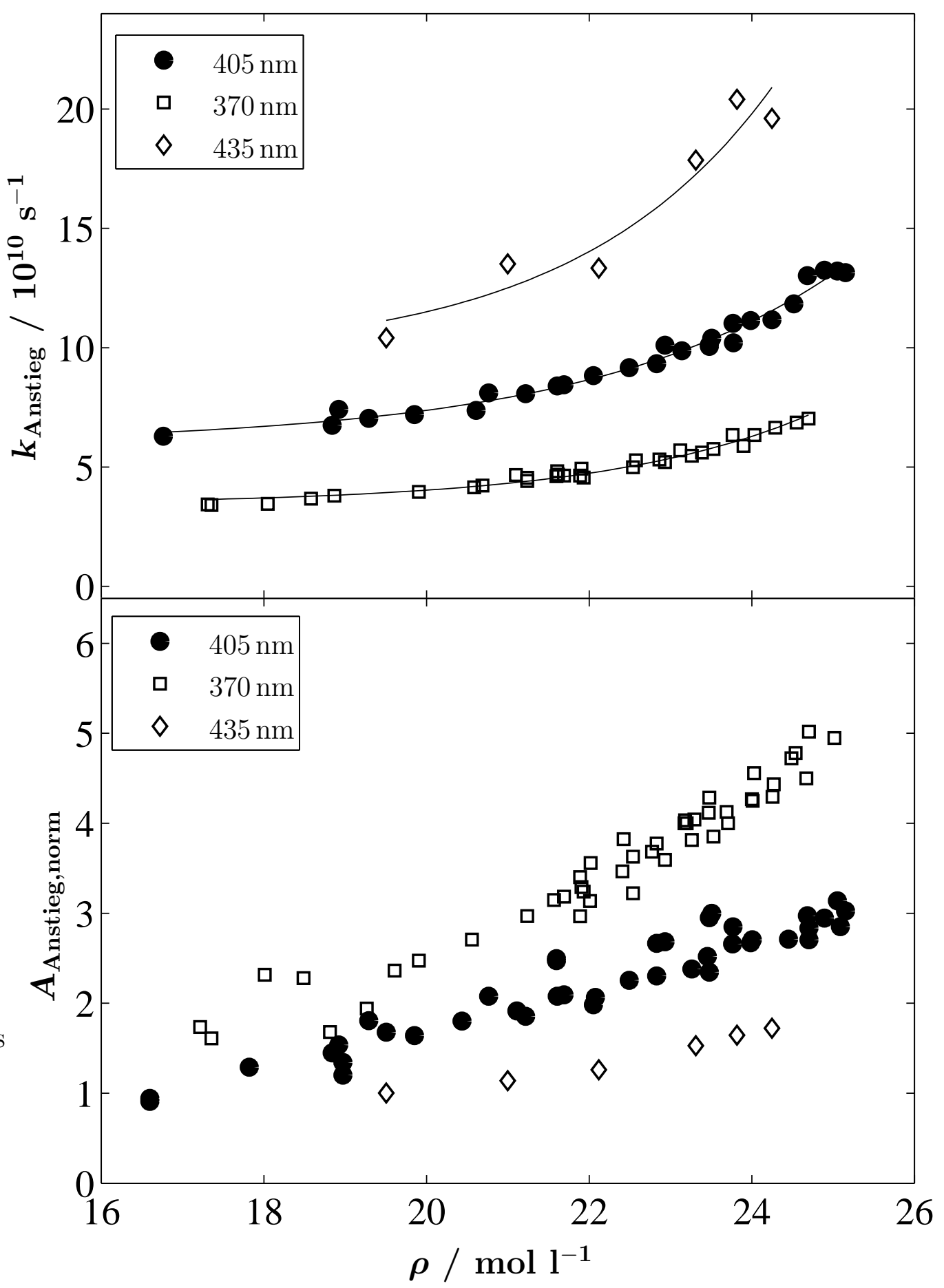

Abbildung 4.15: Dichteabhängigkeit der Geschwindigkeitskonstanten $k_{\text {Anstieg }}$ und der normierten Amplitude $A_{\text {Anstieg,norm }}$ des Anstiegs in überkritischem $\mathrm{CO}_{2}$ bei $304 \mathrm{~nm}$ Anregung verschiedenen Probewellenlängen. 


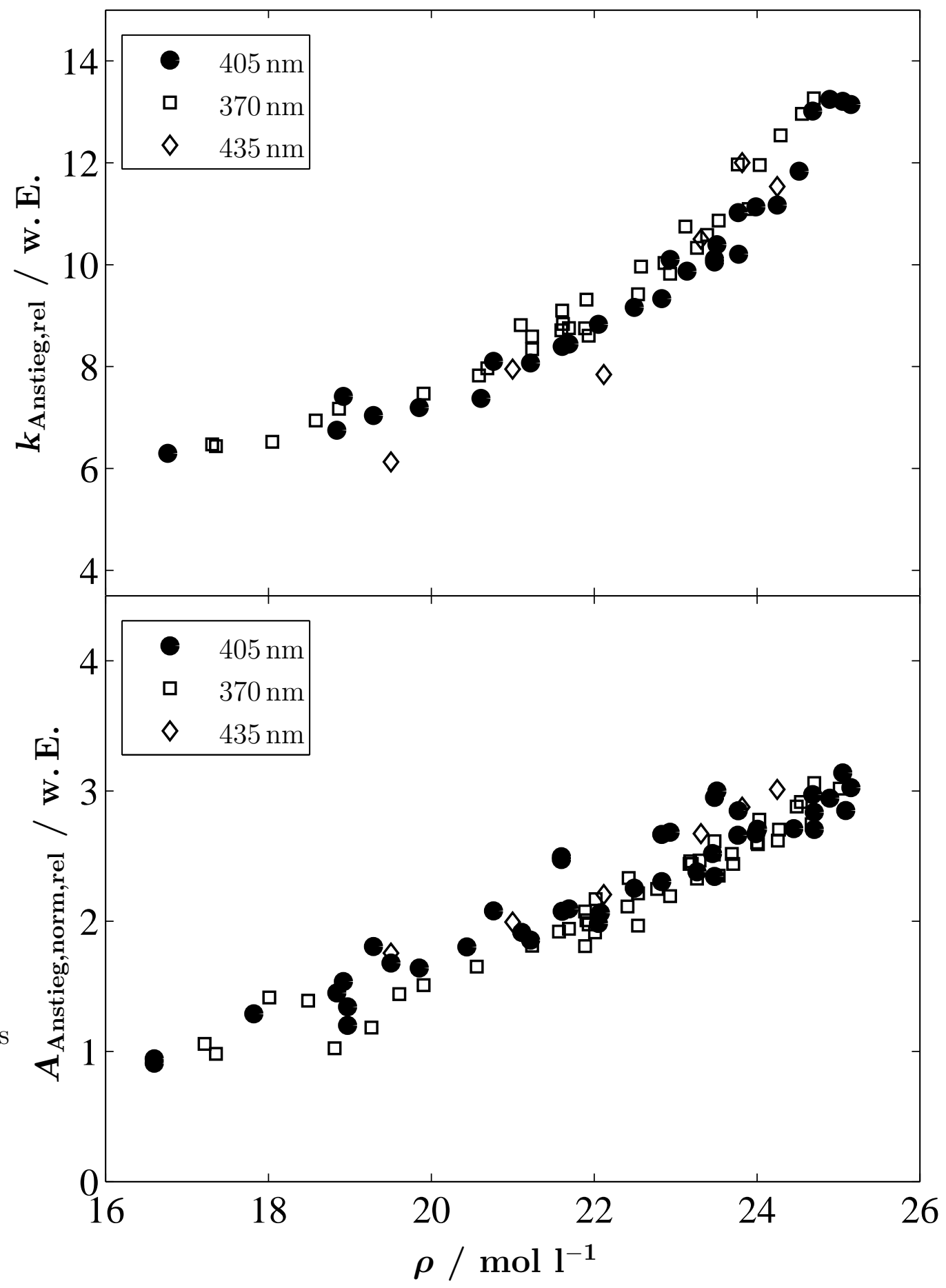

Abbildung 4.16: Dichteabhängigkeit der relativen Geschwindigkeitskonstanten $k_{\text {Anstieg,rel }}$ und der relativen normierten Amplituden $A_{\text {Anstieg,norm,rel }}$ des Anstiegs in überkritischem $\mathrm{CO}_{2}$ bei $304 \mathrm{~nm}$ Anregung und verschiedenen Probewellenlängen. 


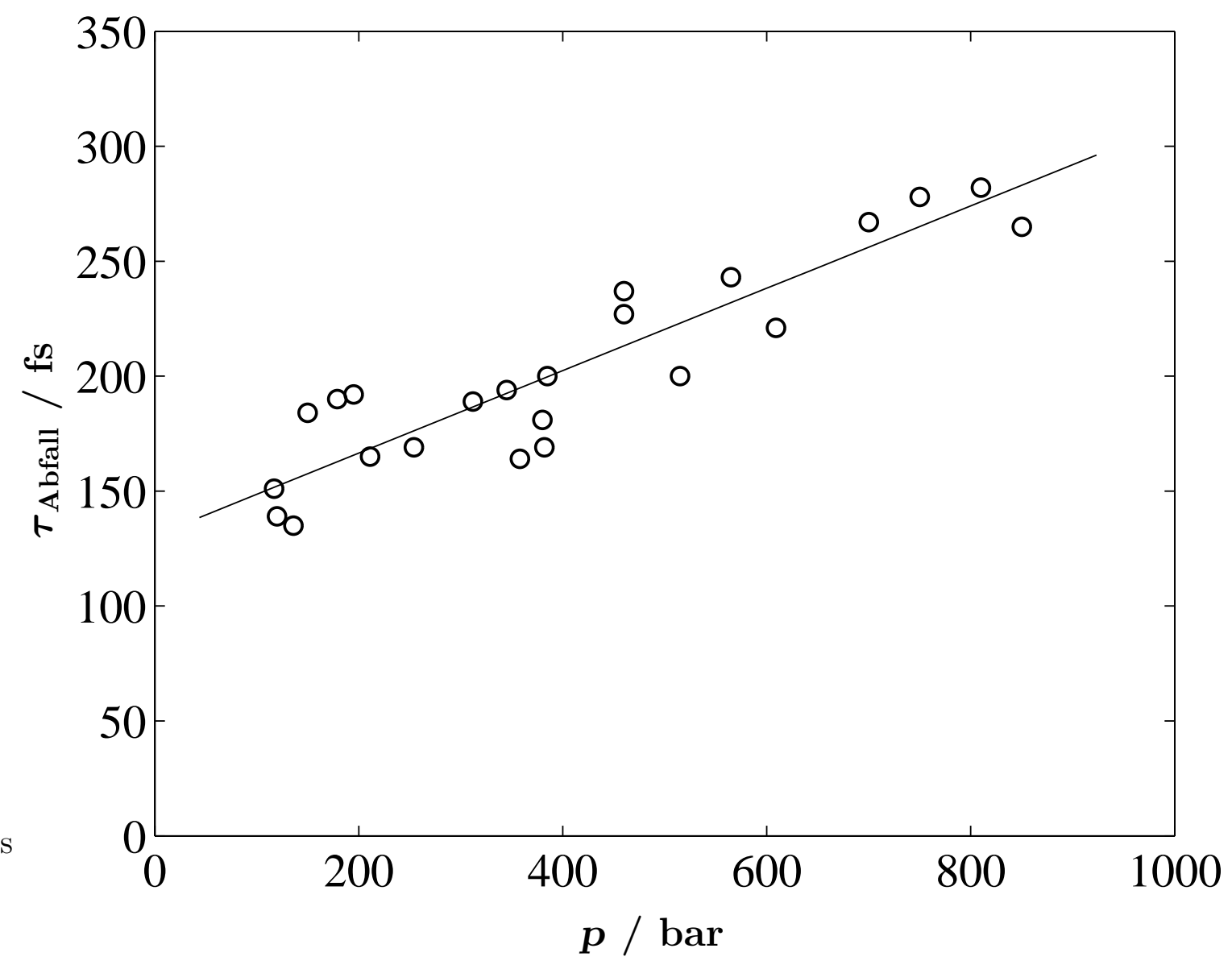

Abbildung 4.17: Druckabhängigkeit der Zeitkonstanten des schnellen Abfalls $\tau_{\text {Abfall }}$ in überkritischem $\mathrm{CO}_{2}(308 \mathrm{~K})$ bei $304 \mathrm{~nm}$ Anregung und $370 \mathrm{~nm}$ Abfrage.

lativen Geschwindigkeitskonstanten, innerhalb experimenteller Fehler, ein identisches Dichteverhalten zeigen (siehe Abbildung 4.16). Dazu wurden die Geschwindigkeitskonstanten mit den Faktoren 1 bei $405 \mathrm{~nm}, 0,53$ bei $370 \mathrm{~nm}$ und 1,7 bei $435 \mathrm{~nm}$ aufeinander skaliert.

Bei einer Probewellenlänge von $370 \mathrm{~nm}$ kann auf Grund einer größeren absoluten Signalhöhe und damit eines besseren Signal- zu Rauschverhältnisses auch eine Druckabhängigkeit der ersten schnellen Abklingzeit $\tau_{\text {Abfall }}$ ermittelt werden. Bei Auftragung dieser Zeitkonstanten gegen den Druck wird ein nahezu linear ansteigendes Verhalten der Zeitkonstanten mit dem Druck beobachtet (siehe Abbildung 4.17).

Dichteabhängigkeit der Spektren Für die Untersuchungen der Dichteabhängigkeit der spektralen Entwicklung wurden, bei zwei verschiedenen Drücken (400 bar und 
800 bar) nach dem in Abschnitt 4.2.2 beschriebenen Verfahren, zeitabhängige Spektren rekonstruiert. Diese Spektren sind zum Vergleich in Abbildung 4.18 dargestellt.

Der Auftragung ist zu entnehmen, dass alle wesentlichen Charakteristika der spektralen Dynamik erhalten bleiben. Die isosbestische Region kann noch weiter auf den Bereich um $405 \mathrm{~nm}$ eingegrenzt werden. Das zeitlich ansteigende Verhalten im kürzerwelligen und das zeitlich abfallende Verhalten im längerwelligeren Spektralbereich sind noch stärker ausgeprägt, woraus auf eine noch schnellere Dynamik des Spektrums geschlossen werden kann.

\section{Anisotropie}

Für die Aufklärung, welche Spezies im kurzen Zeitbereich nach der Anregung einen Beitrag zur Absorption liefern, wurden transiente Anisotropiemessungen durchgeführt. Das Prinzip der Messungen ist in Abschnitt 3.4.1 ausführlich beschrieben. Die Einzelmessungen wurden derart korrigiert, dass die Endamplitude $A_{\text {Anstieg}}$, und der Anfangsoffset $I_{1}$ der parallelen Messung und der senkrechten Messung übereinander lagen. Ferner wurde die Anisotropie erst nach dem ersten schnellen Maximum berechnet, um Beiträge von evtl. auftretenden, kohärenten Lösungsmittelantworten zu vernachlässigen. Ein Beispiel für eine Messung bei parallel und senkrecht polarisierter Anregungs/Abfrageanordnung findet sich in Abbildung 4.19.

Die Anisotropie der Signale wurde für verschiedene Lösungsmitteldichten berechnet und wie in Abbildung 4.19 beispielhaft dargestellt, mit einer einfach exponentiell abfallenden Funktion angepasst. Eine Auftragung der so erhaltenen Orientierungsrelaxationszeit $\tau_{\text {or }}$ gegen die Dichte zeigt Abbildung 4.20.

Die Zeitkonstante steigt dabei mit zunehmender Dichte an, was auf einen Einfluss der Reibung auf die Rotationsrelaxation der betreffenden Spezies hindeutet. Der Anfangswert der Anisotropien beginnt zumeist unter dem theoretisch zu erwartenden Wert bei paralleler Lage der beteiligten Übergangsdipolmomente von 0,4. Dieses könnte mehrere Ursachen haben. Zum einen wurde die Anisotropie erst nach dem ersten Maximum berechnet und zum anderen kann keine genaue Aussage über die Lage der Übergangsdipolmomente der Spezies gemacht werden, die diese Rotationsrelaxation zeigt. Außerdem ist die Anfangsanisotropie kleiner, wenn mehrere Spezies zum transienten Anfangssignal beitragen. 


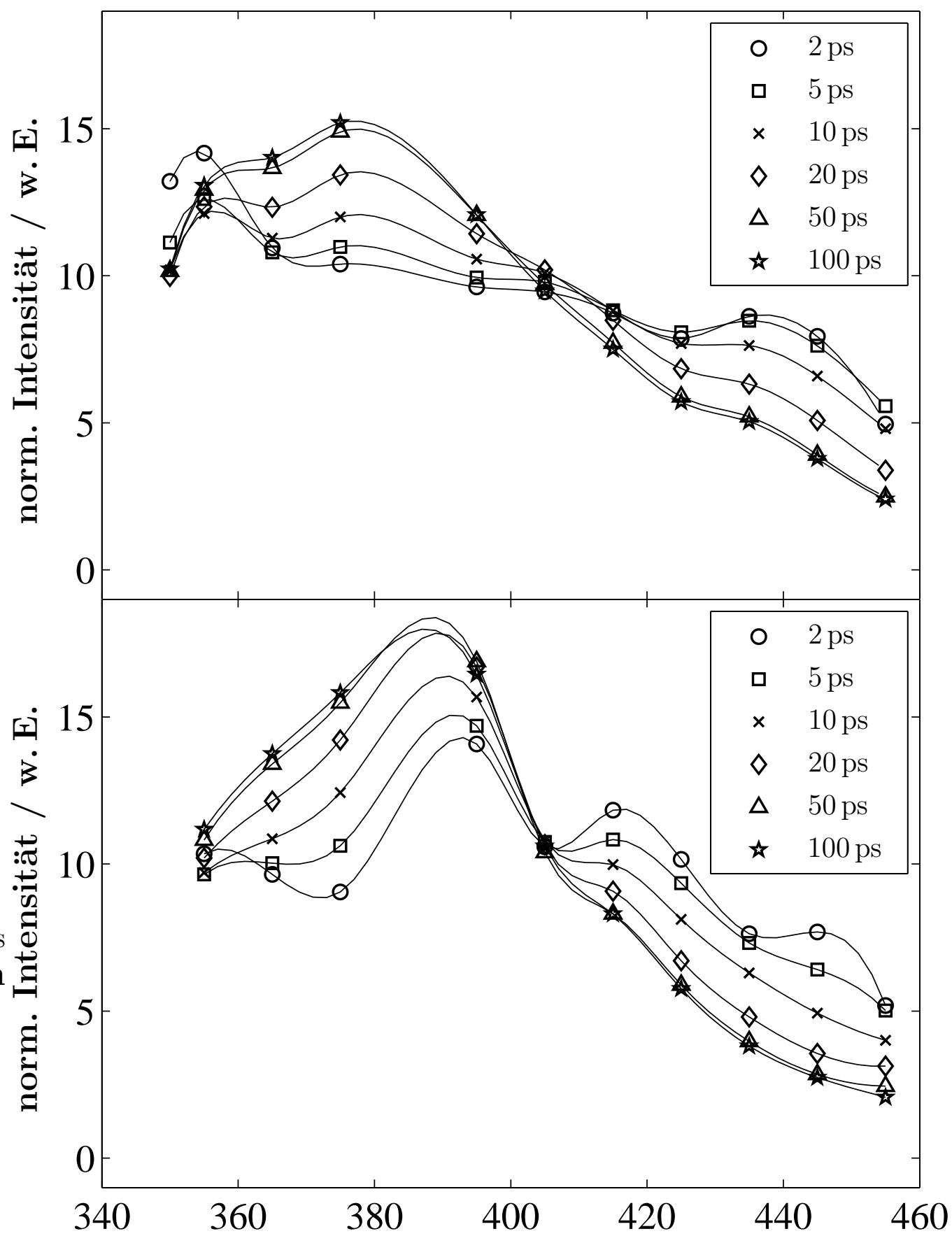

Abbildung 4.18: Auf Fläche normiertes, transientes Absorptionsspektrum in überkritischem $\mathrm{CO}_{2}(308 \mathrm{~K})$ bei verschiedenen Drücken, 400 bar (oben) und 800 bar (unten), bei $304 \mathrm{~nm}$ Anregung zu langen Zeiten. 


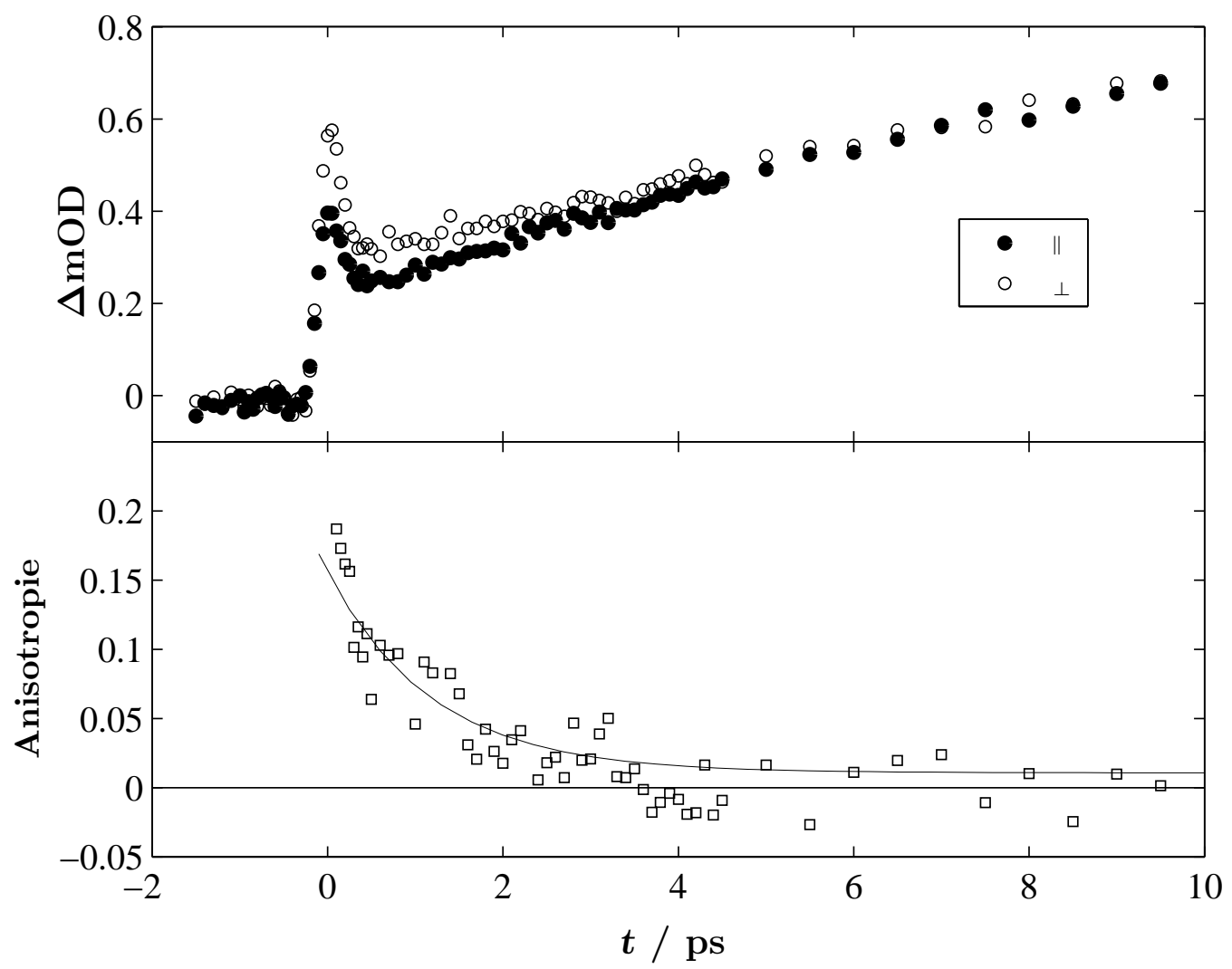

Abbildung 4.19: Beispiel einer Anisotropiemessung in überkritischem $\mathrm{CO}_{2}$ (144 bar, $308 \mathrm{~K}$ ) bei $304 \mathrm{~nm}$ Anregung und $370 \mathrm{~nm}$ Abfrage.

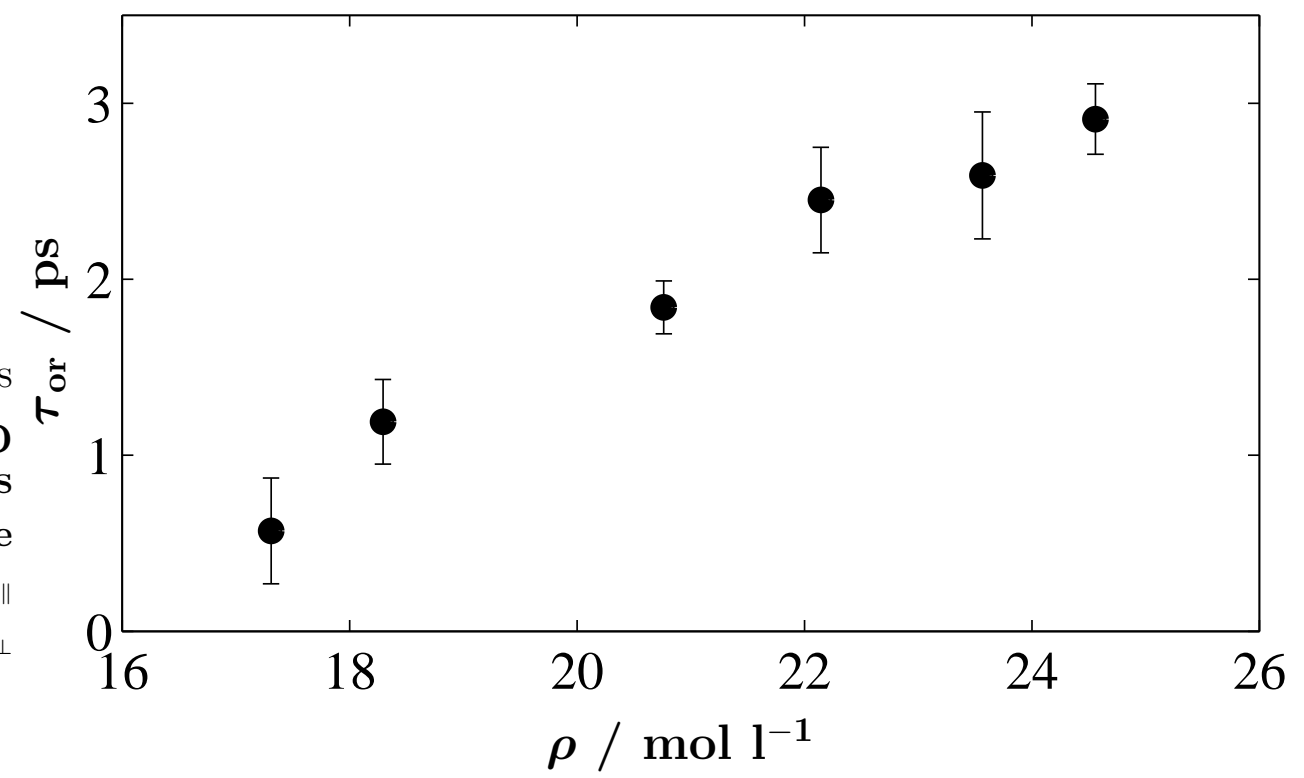

Abbildung 4.20: Dichteabhängigkeit der Orientierungsrelaxationszeiten $\tau_{\text {or }}$ aus Anisotropiemessungen in überkritischem $\mathrm{CO}_{2}(308 \mathrm{~K})$ bei $304 \mathrm{~nm}$ Anregung und $370 \mathrm{~nm}$ Abfrage. 

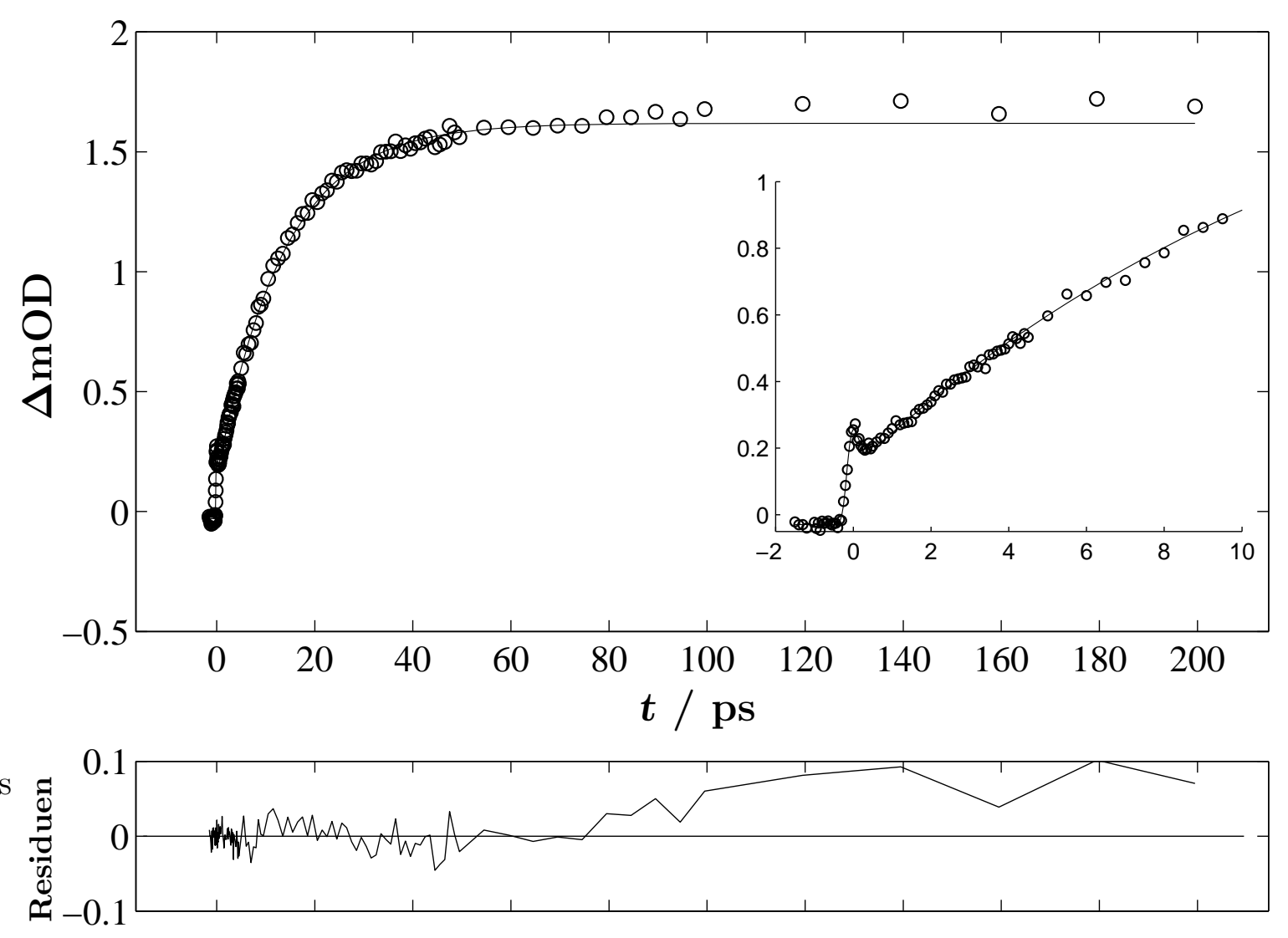

Abbildung 4.21: Typisches Signal in überkritischem $\mathrm{CHF}_{3}$ (505 bar, $310 \mathrm{~K}$ ) bei $304 \mathrm{~nm}$ Anregung und $405 \mathrm{~nm}$ Abfrage.

\subsubsection{Zeitaufgelöste Messungen in überkritischem $\mathrm{CHF}_{3}$}

Im nächsten Abschnitt werden Messungen in überkritischem Fluoroform $\left(\mathrm{CHF}_{3}\right)$ vorgestellt. Fluoroform wurde als Lösungsmittel ausgewählt, um Einflüsse der Polarität des Lösungsmittels auf den Mechanismus der Isomerisierung aufzuklären. $\mathrm{CHF}_{3}$ ist wie $\mathrm{CO}_{2}$ nicht giftig und genauso gut experimentell handhabbar. Die kritische Temperatur beträgt 299,3 K und der kritische Druck 48,3 bar [140]. Es verfügt in der Gasphase über ein permanentes Dipolmoment von 1,65 D [147]. Die Dielektrisitätskonstante $\epsilon$ von $\mathrm{CHF}_{3}$ nimmt mit zunehmender Dichte ebenfalls zu $[147,148]$ und kann theoretisch modelliert werden [149]. In Abbildung 4.21 findet sich eine typische Messung in überkritischem $\mathrm{CHF}_{3}$. Darin ist zu langen Zeiten (>80 ps) ein deutlich abweichender Trend der Anpassung von den Messwerten zu erkennen. Dies ist durch das, von der Verschiebebühne verursachte, Herauswandern des Probestrahls aus der Anregungsregion zu erklären, jedoch konnte diesen Abweichungen im Allgemeinen durch genaue Justage entgegengewirkt werden. 


\section{Spektrale Entwicklung}

Das transiente Spektrum in $\mathrm{CHF}_{3}$ zeigt im Bereich von $370 \mathrm{~nm}$ ein zeitliches Anwachsen einer spektralen Bande. Unter der Annahme, das die zeitliche Entwicklung für Zeiten größer als 2 ps im Wesentlichen von der Population nur einer Spezies herrührt, kann das Spektrum auf diese Population normiert werden. Ein solches auf Fläche normiertes, zeitabhängiges Spektrum findet sich in Abbildung 4.23.

Der zeitliche Verlauf des normierten Spektrum ist durch ein zeitliches Anwachsen der Bande bei $380 \mathrm{~nm}$ und ein zeitliches Abfallen der Flanke ab $420 \mathrm{~nm}$ gekennzeichnet. Bei einer Verzögerungszeit von 2 ps ist analog zum $\mathrm{CO}_{2}$-Spektrum eine schmale Bande bei $355 \mathrm{~nm}$ erkennbar, jedoch ist zu längeren Verzögerungszeiten die daraus resultierende Schulter nicht so stark ausgeprägt wie im $\mathrm{CO}_{2}$-Spektrum. Ferner ist im normierten Spektrum eine isosbestische Region zu erkennen, die im Bereich um $410 \mathrm{~nm}$ lokalisiert ist. Das Spektrum scheint in $\mathrm{CHF}_{3}$ gegenüber dem Spektrum in $\mathrm{CO}_{2}$ etwas rotverschoben zu sein. Der gesamte zeitliche Verlauf des transienten Spektrums (Abbildung 4.22) und des normierten Spektrums (Abbildung 4.23) ist denen in überkritischen $\mathrm{CO}_{2}$ ansonsten sehr ähnlich (vergleiche Abbildung 4.7 und 4.8).

\section{Dichteabhängigkeiten}

In diesem Abschnitt werden die Ergebnisse druckabhängiger transienter Absorptionsmessungen in überkritischem $\mathrm{CHF}_{3}$ vorgestellt (siehe auch Anhang A.2.2). Diese Messungen sind in der isosbestischen Region (siehe Abschnitt 4.2.3) bei einer Probewellenlänge von $410 \mathrm{~nm}$ durchgeführt worden.

Eine Auftragung der ermittelten Zeitkonstanten des monoexponentiellen Anstiegs $\tau_{\text {Anstieg }}$ und der zugehörigen Endamplitude $A_{\text {Anstieg }}$ des Signals findet sich in Abbildung 4.24 .

Die Auftragung der Zeitkonstanten gegen den Druck (siehe Abbildung 4.24) zeigt ein exponentiell abklingendes Verhalten der Zeitkonstanten mit zunehmenden Druck. Gleichzeitig steigt die zugehörige Amplitude monoton mit dem Druck an. Eine funktionelle Abhängigkeit kann aus den vorhandenen Daten nur schwer abgeschätzt werden.

Bei einer Auftragung der Geschwindigkeitskonstanten $k_{\text {Anstieg }}$ und der Amplitude für den Anstieg gegen die Dichte wird der in Abbildung 4.25 gezeigte Verlauf erhalten. Die Umrechnung der Druck- in eine Dichteskala erfolgte nach dem in Anhang A.3.2 beschriebenem Verfahren.

Der Auftragung ist zu entnehmen, dass die Geschwindigkeitskonstante eher exponentiell mit zunehmender Dichte ansteigt, wobei die zugehörige Amplitude des Signals eher linear mit der Dichte skaliert. 


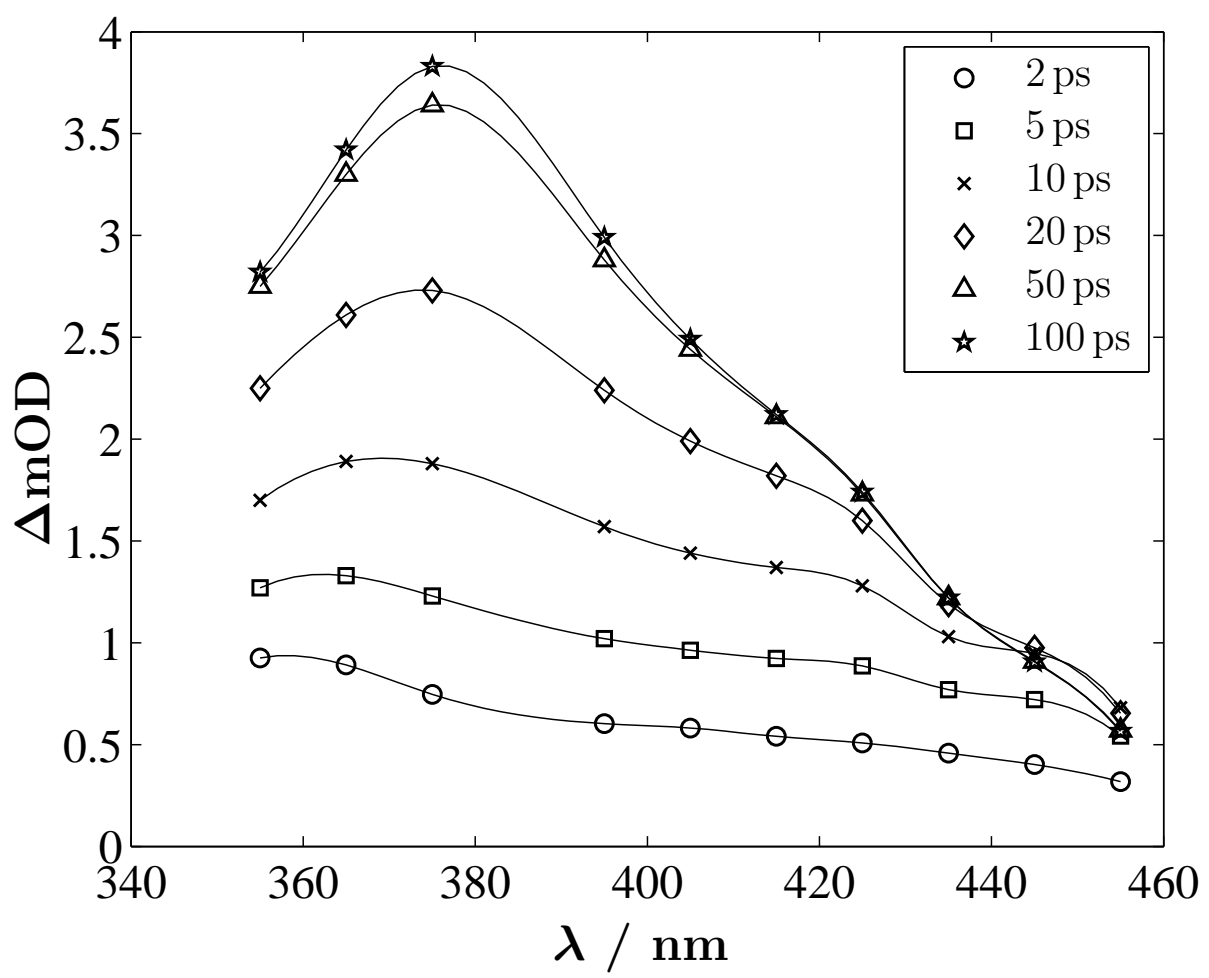

Abbildung 4.22: Transientes Absorptionsspektrum in überkritischem $\mathrm{CHF}_{3}(310 \mathrm{~K}$, 510 bar) bei $304 \mathrm{~nm}$ Anregung zu langen Zeiten.

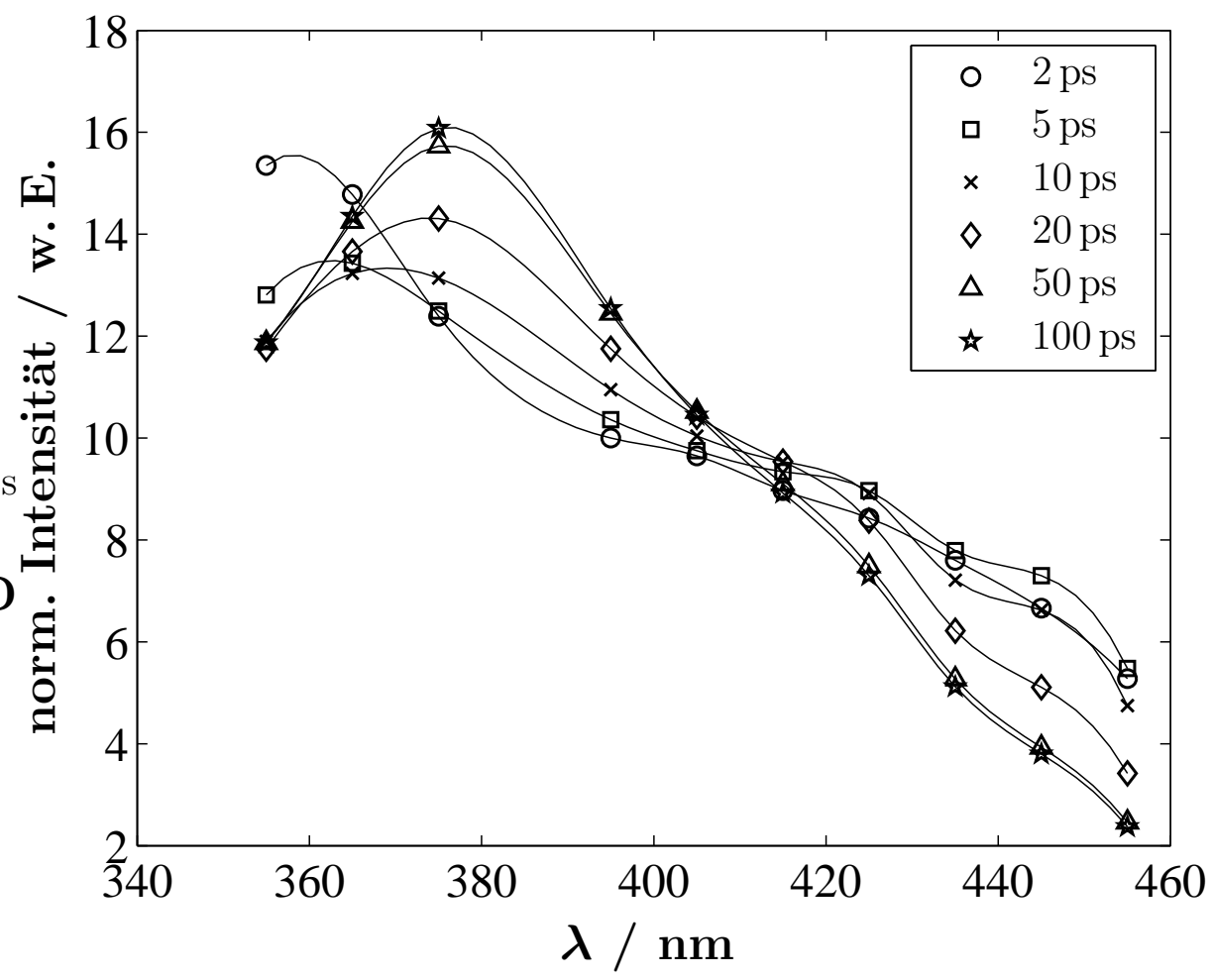

Abbildung 4.23: Auf Fläche normiertes, transientes Absorptionsspektrum in überkritischem $\mathrm{CHF}_{3}(310 \mathrm{~K}, 510$ bar) bei 304 nm Anregung zu langen Zeiten. 


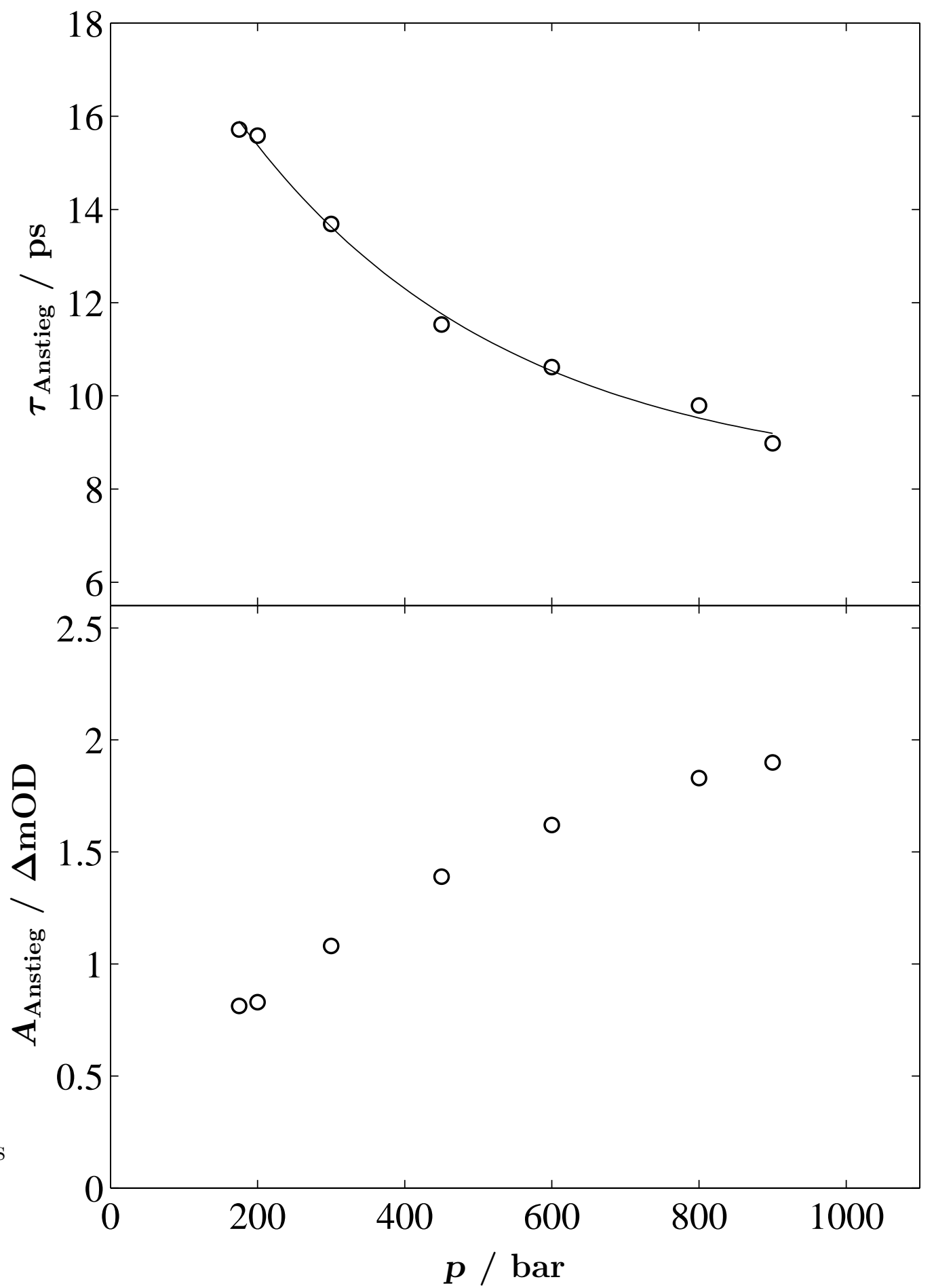

Abbildung 4.24: Druckabhängigkeit der Zeitkonstanten des Anstiegs $\tau_{\text {Anstieg }}$ und der Amplitude $A_{\text {Anstieg }}$ in überkritischem $\mathrm{CHF}_{3}(310 \mathrm{~K})$ bei $315 \mathrm{~nm}$ Anregung und $410 \mathrm{~nm}$ Abfrage. 


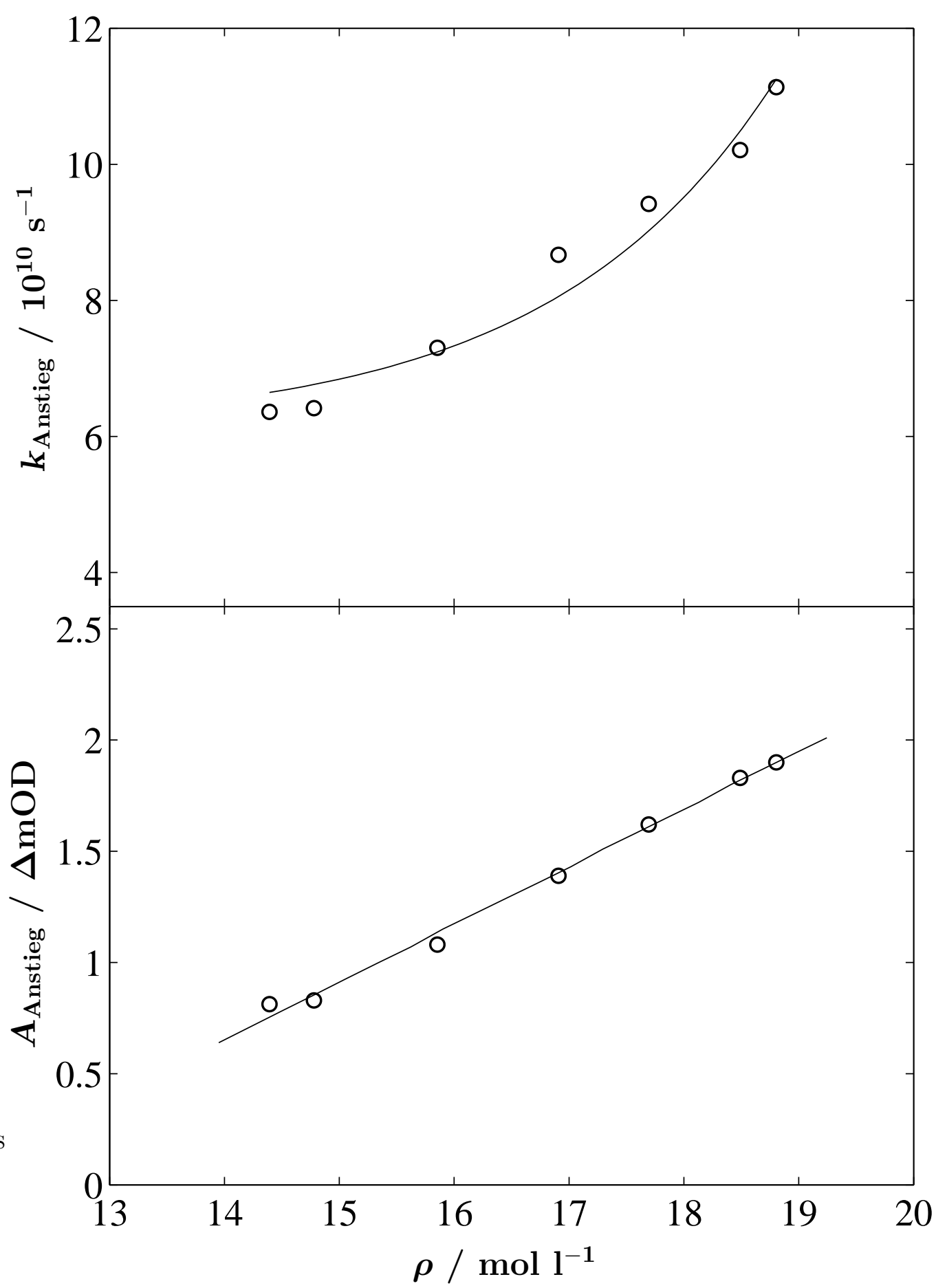

Abbildung 4.25: Druck- bzw. Dichteabhängigkeit der Geschwindigkeitskonstanten des Anstiegs $k_{\text {Anstieg }}$ und der Amplitude $A_{\text {Anstieg }}$ in überkritischem $\mathrm{CHF}_{3}$ $(310 \mathrm{~K})$ bei $315 \mathrm{~nm}$ Anregung und $410 \mathrm{~nm}$ Abfrage. 

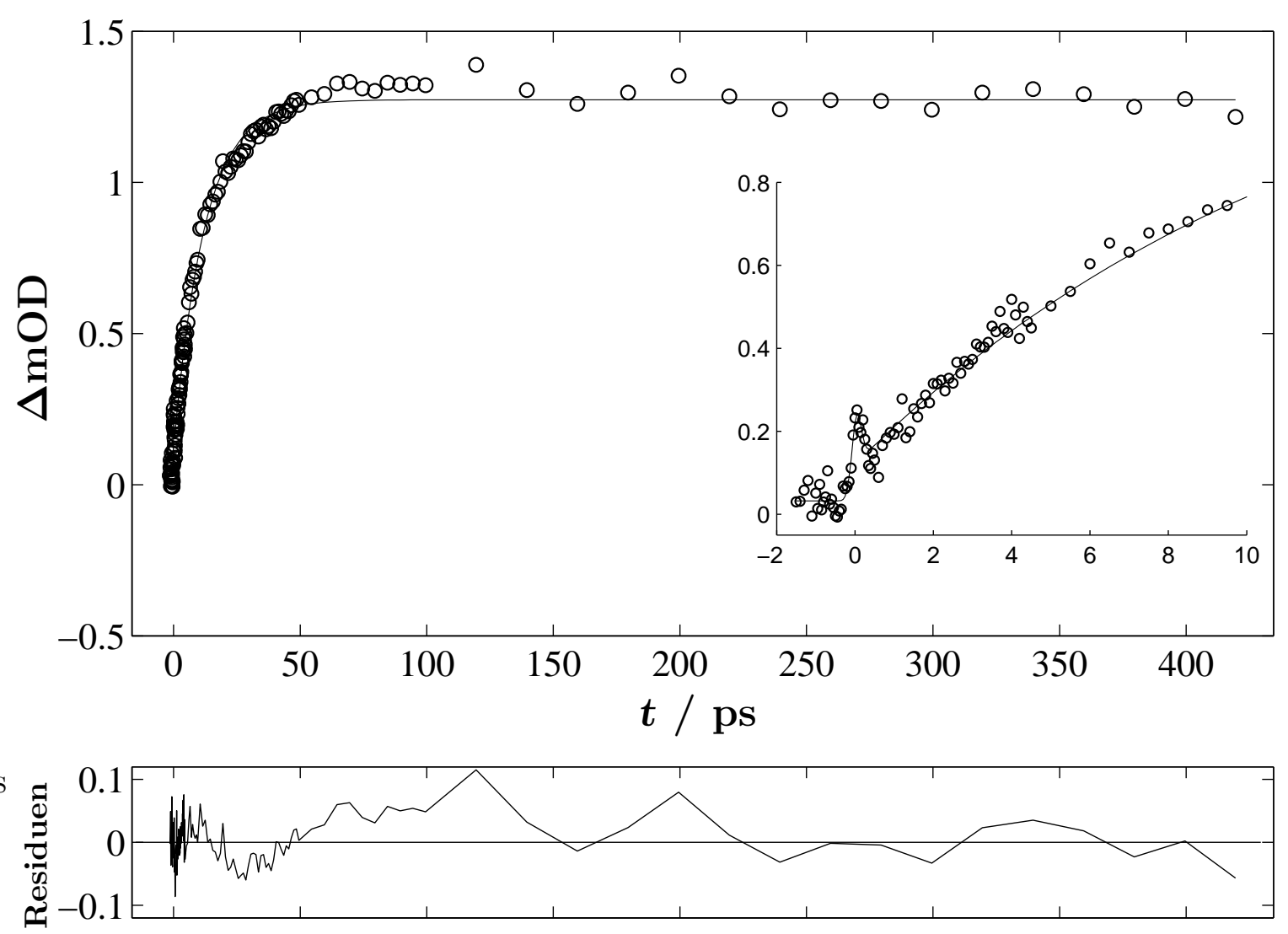

Abbildung 4.26: Typisches Signal in überkritischem Ethan (350 bar, $310 \mathrm{~K}$ ) bei $315 \mathrm{~nm}$ Anregung und $400 \mathrm{~nm}$ Abfrage.

\subsubsection{Zeitaufgelöste Messungen in überkritischem Ethan}

Dieser Abschnitt gibt einen Überblick über die zeitaufgelösten Messungen in überkritischem Ethan. Ethan wurde als ein Vertreter für Alkane als Lösungsmittel ausgewählt. Es zeichnet sich genauso wie die davor verwendeten Lösungsmittel $\mathrm{CO}_{2}$ und $\mathrm{CHF}_{3}$ durch eine leichte experimentelle Handhabbarkeit aus. Die kritische Temperatur liegt bei 305,3 K, der kritische Druck beträgt 48,7 bar und es ist unpolar. Für ein Beispiel einer zeitaufgelösten Messung in überkritischem Ethan siehe Abbildung 4.26.

\section{Spektrale Entwicklung}

Zunächst wurden wiederum, bei verschiedenen spektralen Positionen bei gleicher Anregungswellenlänge und gleicher Dichte, transiente Einzelmessungen durchgeführt. Daraus wurde ein, in Abbildung 4.27 gezeigtes, Spektrum erhalten. 
Das zeitabhängige Spektrum zeigt ein zeitliches Anwachsen einer bei $370 \mathrm{~nm}$ aufkommenden spektralen Bande. Die Gestalt des Spektrums ist sehr ähnlich zu den in $\mathrm{CO}_{2}$ und $\mathrm{CHF}_{3}$ erhaltenen Spektren.

Durch das Normieren auf die Population, und damit auf die Fläche (siehe Abschnitt 4.2.2), wird eine in Abbildung 4.28 gezeigte spektrale Dynamik erhalten.

Das populationsnormierte Spektrum weist wie bei den anderen verwendeten Lösungsmitteln eine zeitlich anwachsende Bande bei $375 \mathrm{~nm}$ und einen Bereich mit geringer oder keiner spektralen Dynamik auf (isosbestische Region). Jedoch ist dieser Bereich nicht so deutlich einzugrenzen, wie in den anderen Lösungsmitteln. Ferner ist die bereits in den anderen Lösungsmitteln bei einer Verzögerungszeit von 2 ps noch zu beobachtende schmale Bande bei $355 \mathrm{~nm}$ in diesem Fall noch länger erkennbar. Im Gegensatz zu $\mathrm{CO}_{2}$, wo diese später als Schulter erkennbar war, ist sie hier von der breiten Bande bei $370 \mathrm{~nm}$ getrennt. Insgesamt erscheint das Spektrum in Ethan etwas rotverschoben gegenüber dem $\mathrm{CO}_{2}$-Spektrum.

\section{Dichteabhängigkeiten}

Die folgenden druck- bzw. dichteabhängigen Messungen wurden bei einer Probewellenlänge von $405 \mathrm{~nm}$ durchgeführt (siehe auch Anhang A.2.3).

In Abbildung 4.29 sind die Abhängigkeit der Zeitkonstanten des monoexponentiellen Anstiegs $\tau_{\text {Anstieg }}$ und die zugehörige Endamplitude $A_{\text {Anstieg }}$ des Signals in Abhängigkeit des Ethandruckes dargestellt.

Die Zeitkonstante zeigt ein, ähnlich zu den anderen Lösungsmitteln, eher exponentielles Abklingen mit zunehmenden Druck. Der Verlauf der Amplitude ist monoton anwachsend mit steigendem Druck, jedoch lässt sich über einen funktionellen Zusammenhang nur schwer eine Aussage treffen.

Der funktionelle Zusammenhang zwischen der Geschwindigkeitskonstanten und der Dichte des Lösungsmittels ist nahezu exponentiell ansteigend mit zunehmender Dichte (siehe Abbildung 4.30). Für die entsprechende Dichteabhängigkeit der zugehörigen Amplitude lässt sich wiederum kein direkter funktioneller Zusammenhang angeben. Das Verhalten kann wiederum als monoton anwachsend mit steigender Dichte angegeben werden. 


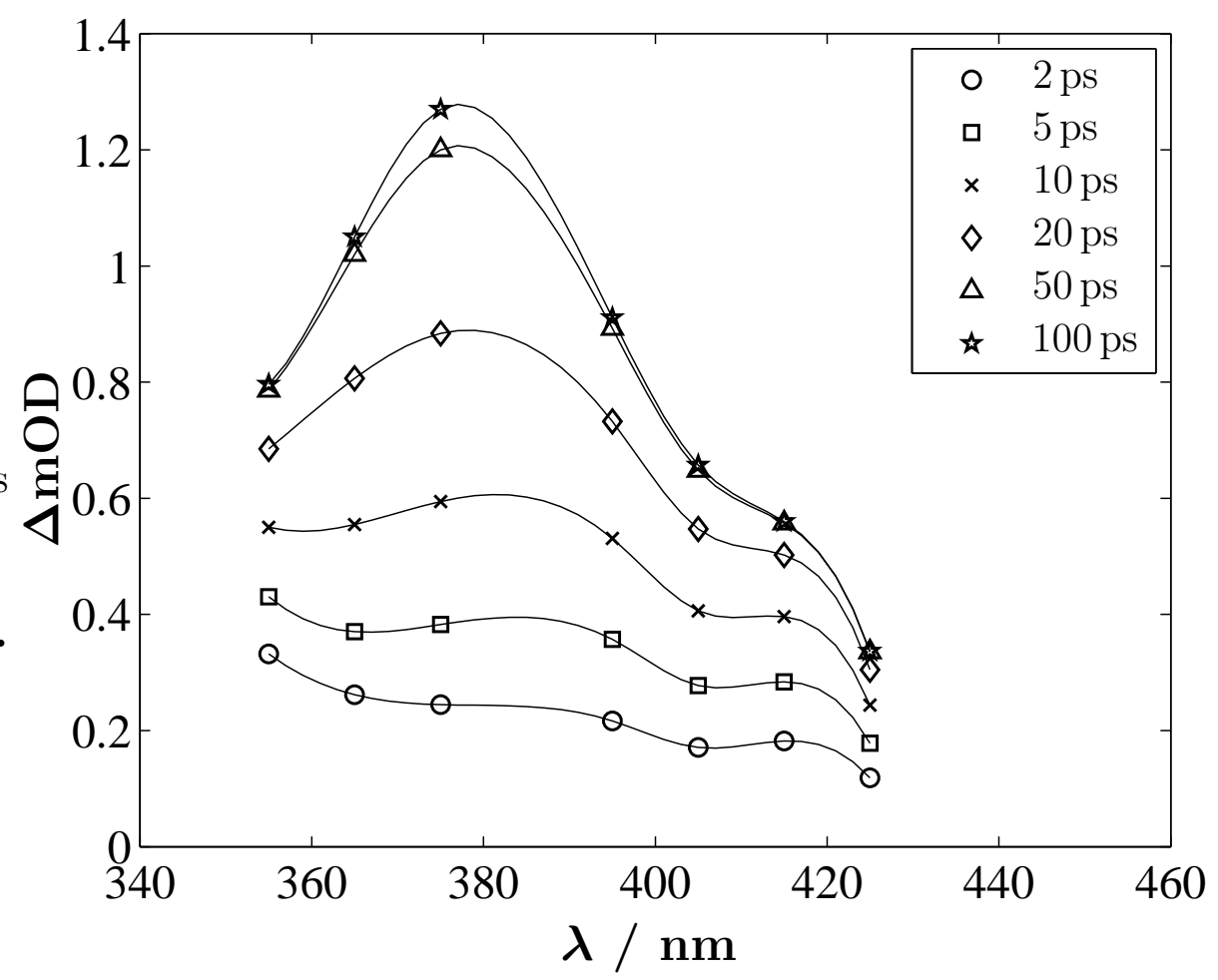

Abbildung 4.27: Transientes Absorptionsspektrum in überkritischem Ethan $(310 \mathrm{~K}$, 400 bar) bei $315 \mathrm{~nm}$ Anregung zu langen Zeiten.

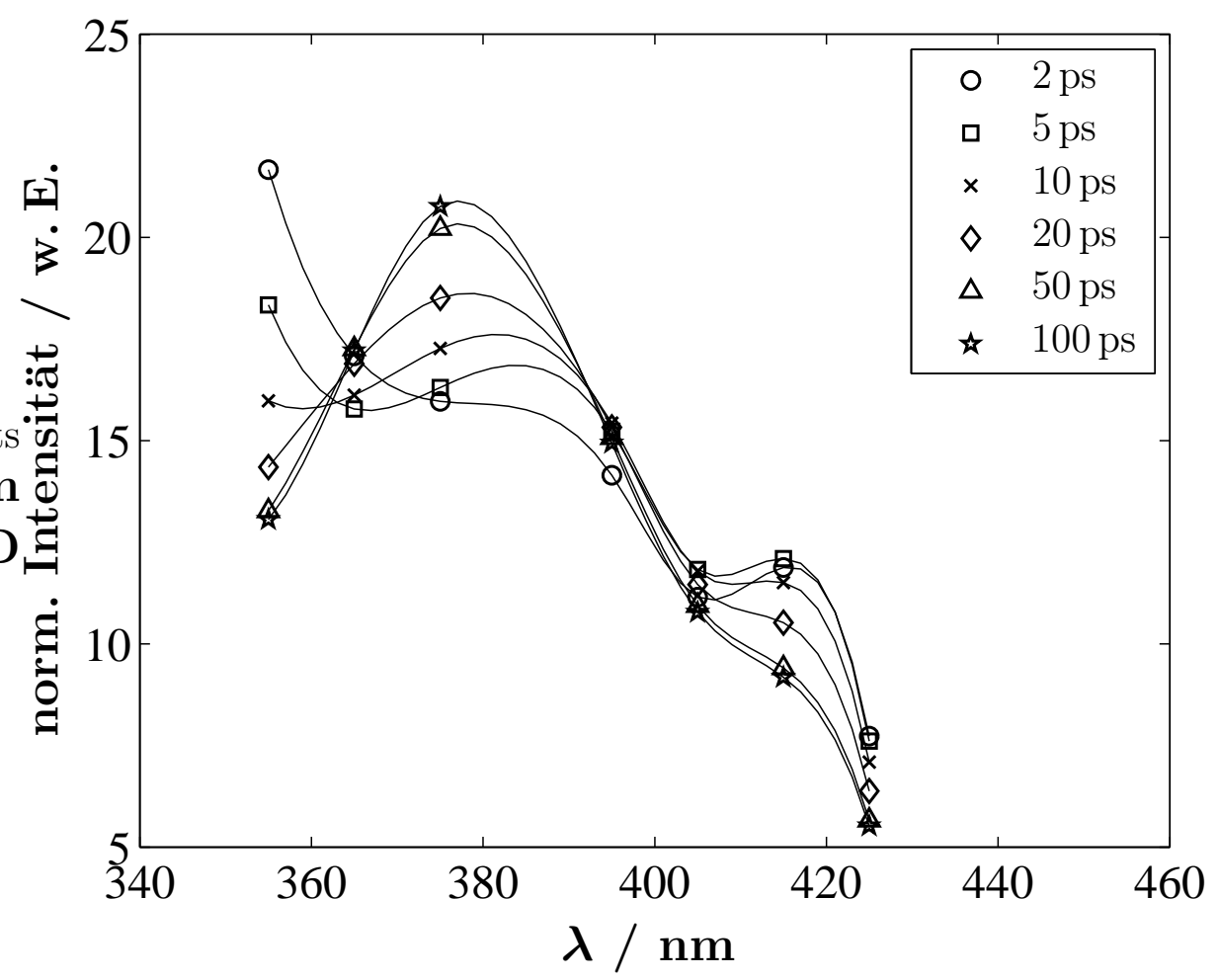

Abbildung 4.28: Auf Fläche normiertes, transientes Absorptionsspektrum in überkritischem Ethan (310 K, 400 bar) bei $315 \mathrm{~nm}$ Anregung zu langen Zeiten. 


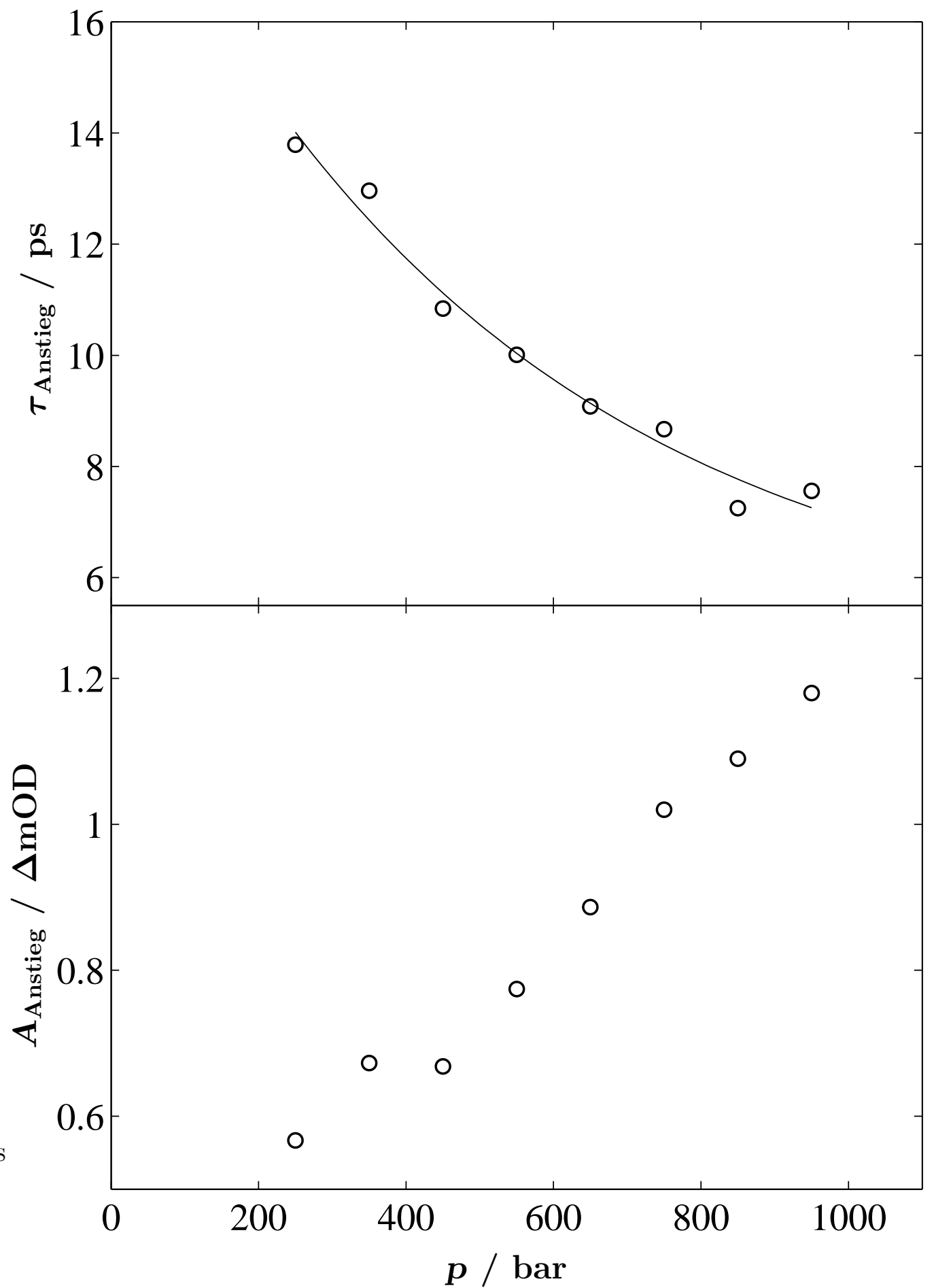

Abbildung 4.29: Druckabhängigkeit der Zeitkonstanten des Anstiegs $\tau_{\text {Anstieg }}$ und der Amplitude $A_{\text {Anstieg }}$ in überkritischem Ethan $(310 \mathrm{~K})$ bei $315 \mathrm{~nm}$ Anregung und $405 \mathrm{~nm}$ Abfrage. 


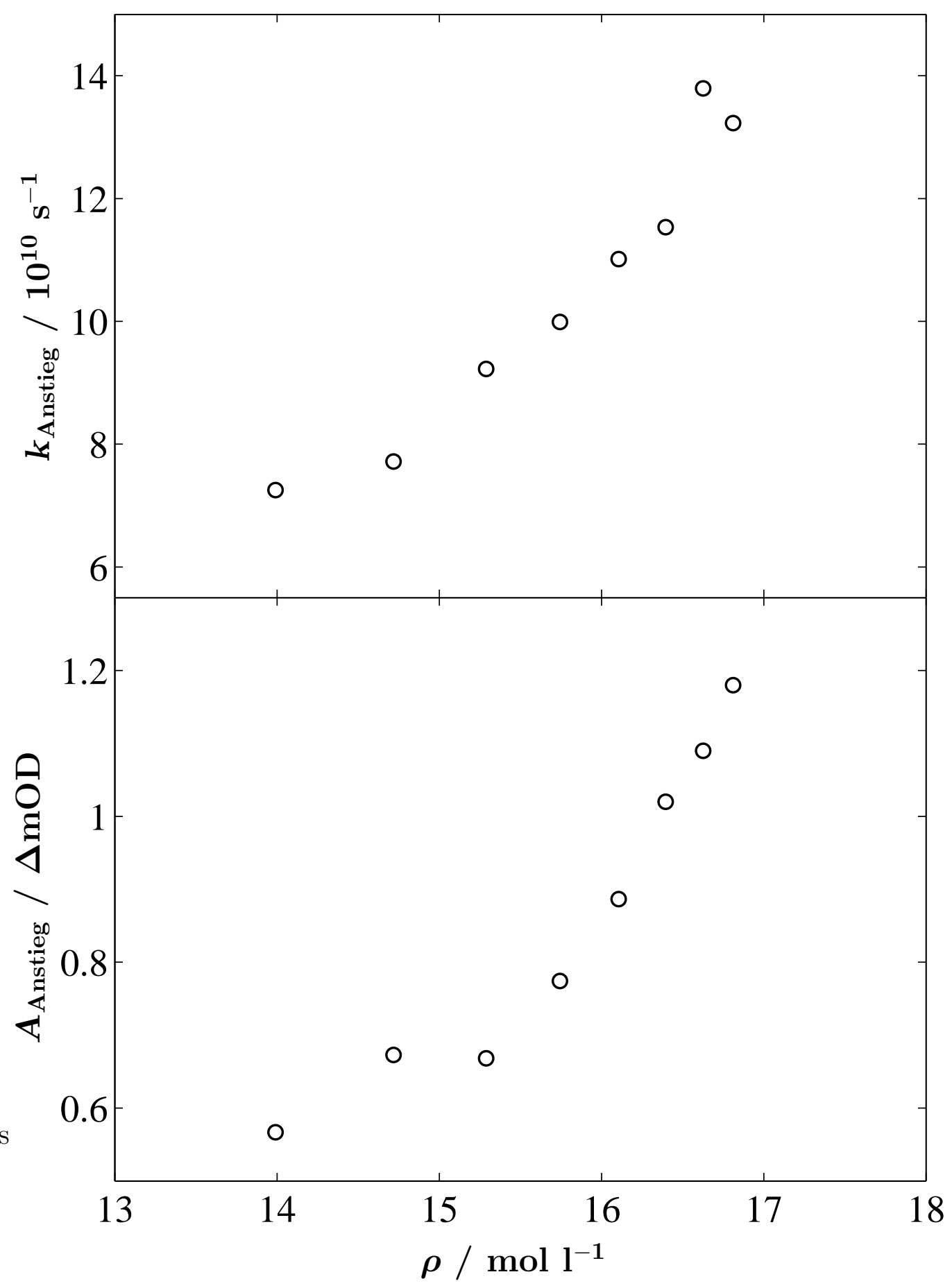

Abbildung 4.30: Druck- bzw. Dichteabhängigkeit der Geschwindigkeitskonstanten des Anstiegs $k_{\text {Anstieg }}$ und der Amplitude $A_{\text {Anstieg }}$ in überkritischem Ethan ( $310 \mathrm{~K})$ bei $315 \mathrm{~nm}$ Anregung und $410 \mathrm{~nm}$ Abfrage. 

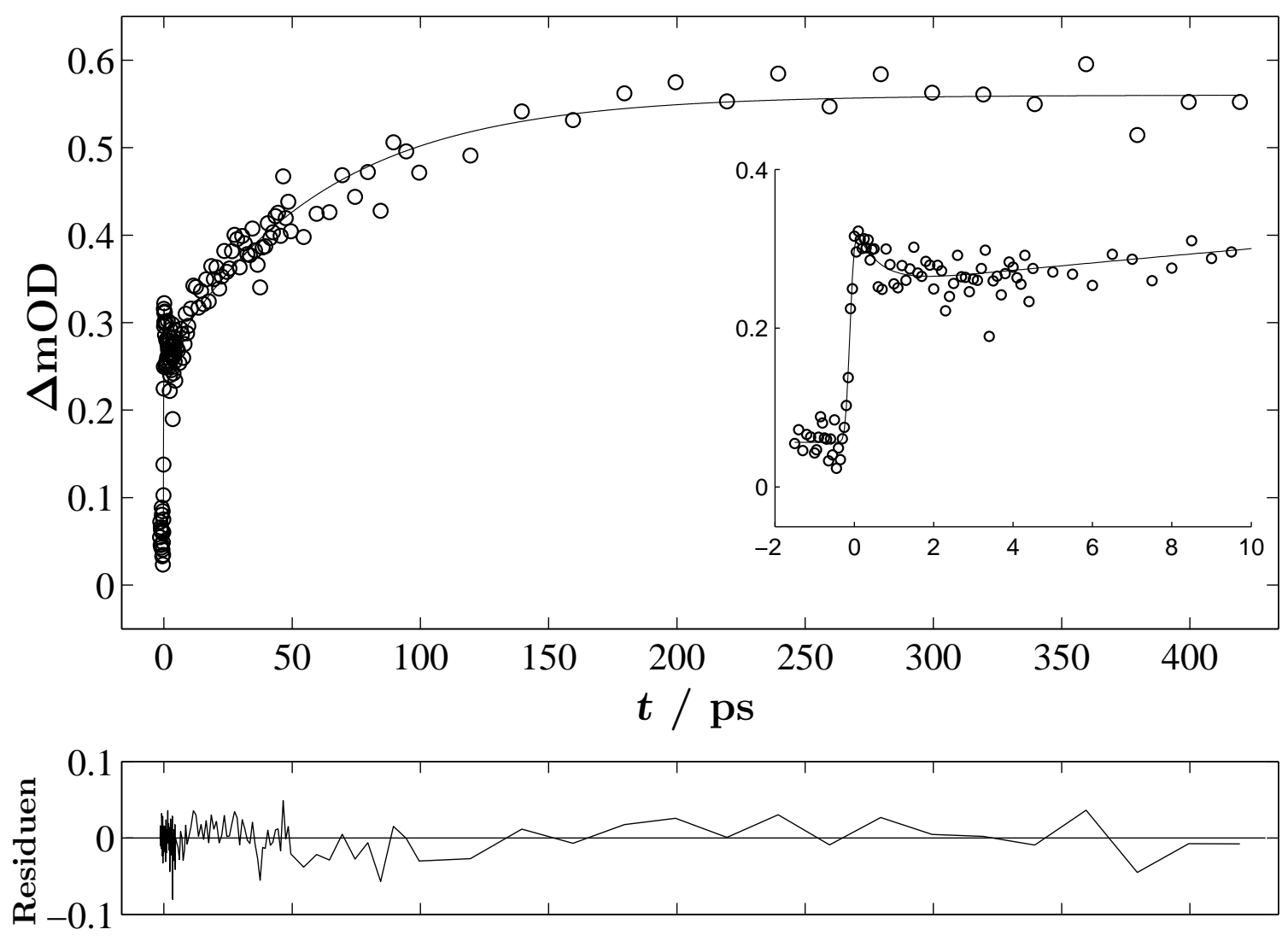

Abbildung 4.31: Typisches Signal in überkritischem Xenon (430 bar, $308 \mathrm{~K}$ ) bei $310 \mathrm{~nm}$ Anregung und $370 \mathrm{~nm}$ Abfrage.

\subsubsection{Zeitaufgelöste Messungen in überkritischem Xenon}

Dieser Abschnitt befasst sich mit ersten zeitaufgelösten Messungen in überkritischem Xenon. Überkritisches Xenon wurde aus folgenden Gründen für die, in diesem Abschnitt gezeigten, und für geplante weitere Untersuchungen (siehe Kapitel 6) ausgewählt: Xenon ist ein atomares Edelgas, dessen kritische Temperatur mit 289,7 K im Bereich der Zimmertemperatur liegt. Mit einem kritischen Druck von 58,4 bar ist es für Messungen im überkritischen Bereich experimentell sehr gut zugänglich. Desweiteren traf die Wahl auf Xenon als Lösungsmittel, da Energietransfer nur in translatorischen Freiheitsgraden stattfinden kann. Es verfügt ferner auf Grund seiner großen Elektronenhülle über eine gute dielektrische Polarisierbarkeit, die der von $\mathrm{CO}_{2}$ sehr ähnlich ist.

Bei ersten zeitaufgelösten Messungen hat sich gezeigt, das die Endamplitude des Signalplateaus $A_{\text {Anstieg }}$ bei gleichen reduzierten Dichten kleiner ist als bei einer direkten 

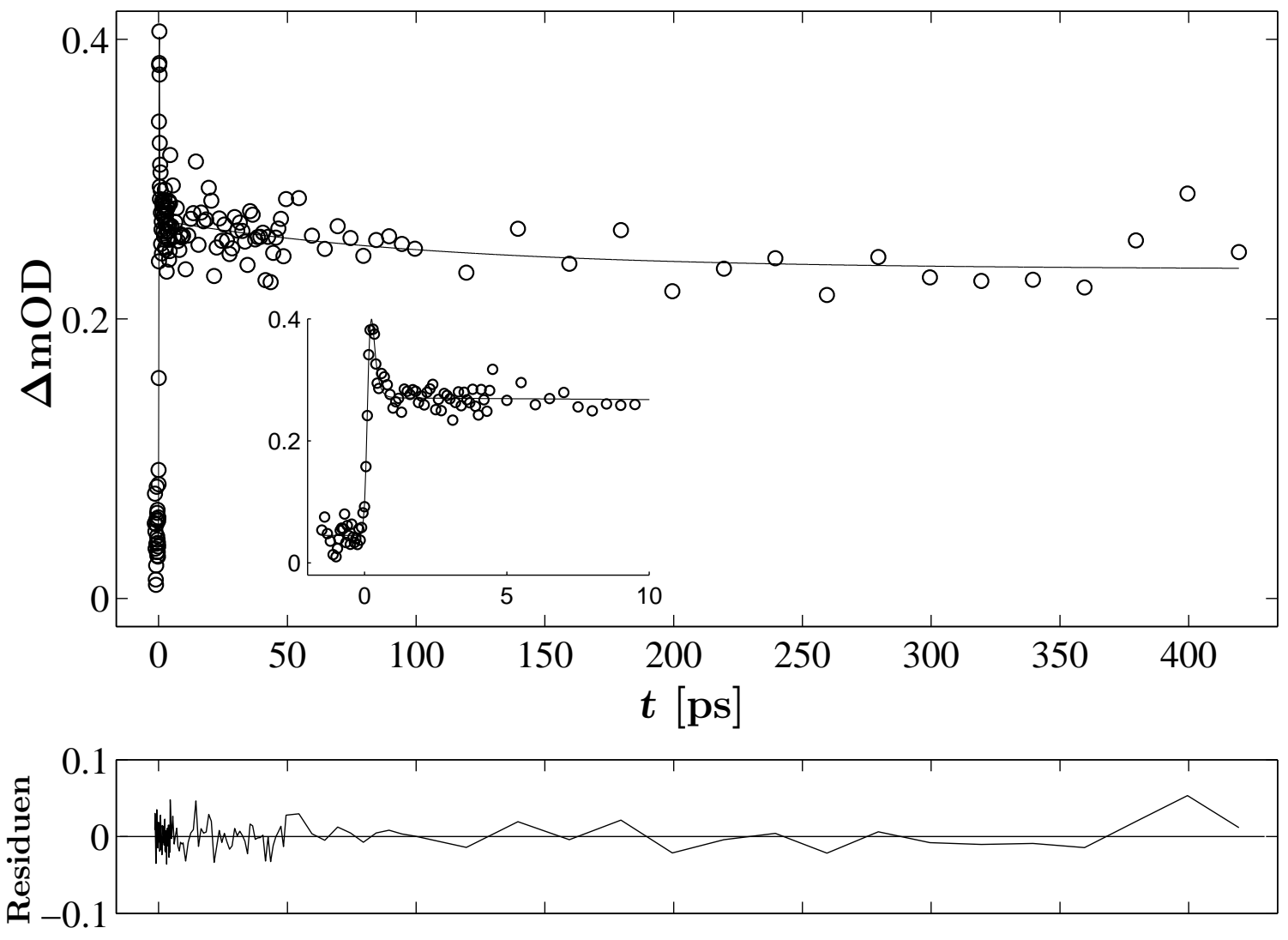

Abbildung 4.32: Transientes Signal in überkritischem Xenon (430 bar, $308 \mathrm{~K}$ ) bei $310 \mathrm{~nm}$ Anregung und $425 \mathrm{~nm}$ Abfrage.

Vergleichsmessung in überkritischem $\mathrm{CO}_{2}$. Ein Beispiel für eine zeitaufgelöste, transiente Absorptionsmessung zeigt Abbildung 4.31.

Das Absorptionszeitprofil (Abbildung 4.31) zeigt außerdem ein sehr viel langsameres Ansteigen des Signals als in den anderen überkritischen Lösungsmitteln. Diese ansteigende Komponente ist bei Wellenlängen $\geq 425 \mathrm{~nm}$ überhaupt nicht mehr zu finden. Stattdessen fällt das Signal, wie in Abbildung $4.32 \mathrm{zu}$ sehen nach dem schnellen Abfall mit einer sehr großen Zeitkonstante langsam ab.

\section{Spektrale Entwicklung}

Wie bei den anderen Lösungsmitteln wurde zunächst ein transientes Spektrum rekonstruiert. Das so erhalten Spektrum ist in Abbildung 4.33 gezeigt.

Durch den bereits erwähnten langsamen Anstieg des transienten Absorptionssignals wächst auch das transiente Spektrum (siehe Abbildung 4.33) noch bis 250 ps weiter 
an. Das Spektrum besteht aus zwei Banden: einer schmalen Bande bei $365 \mathrm{~nm}$ und einer breiteren Bande bei etwa $385 \mathrm{~nm}$. Der zeitliche Verlauf des Spektrums ist bis zu einer Verzögerungszeit von 10 ps ähnlich dem Kurzzeitspektrum in $\mathrm{CO}_{2}$. Ein Anwachsen der Bande bei $385 \mathrm{~nm}$ ist erst ab einer Verzögerungszeit von $50 \mathrm{ps}$ deutlich zu erkennen. Das Xenon-Spektrum ist ebenfalls etwas rotverschoben gegenüber dem $\mathrm{CO}_{2}$-Spektrum. Auch in Xenon wurde das Spektrum auf die Fläche normiert, um die spektrale Dynamik sichtbar zu machen (siehe Abbildung 4.34).

Das normierte Spektrum zeigt ein ähnliches zeitliches Verhalten wie in den anderen Lösungsmitteln, jedoch ist diese Entwicklung sehr viel langsamer. Dies ist ein starker Hinweis, dass die spektrale Dynamik hauptsächlich auf Effekte der Schwingungsenergierelaxation zurückzuführen ist, da der Energietransfer in das Xenon sehr viel ineffektiver sein sollte als in die anderen mehratomigen Lösungsmittel. Ferner ist zu beobachten, dass die schmale Bande bei $365 \mathrm{~nm}$ nach $50 \mathrm{ps}$ eine ähnliche spektrale Dynamik zeigt wie die breite Bande bei $385 \mathrm{~nm}$. 


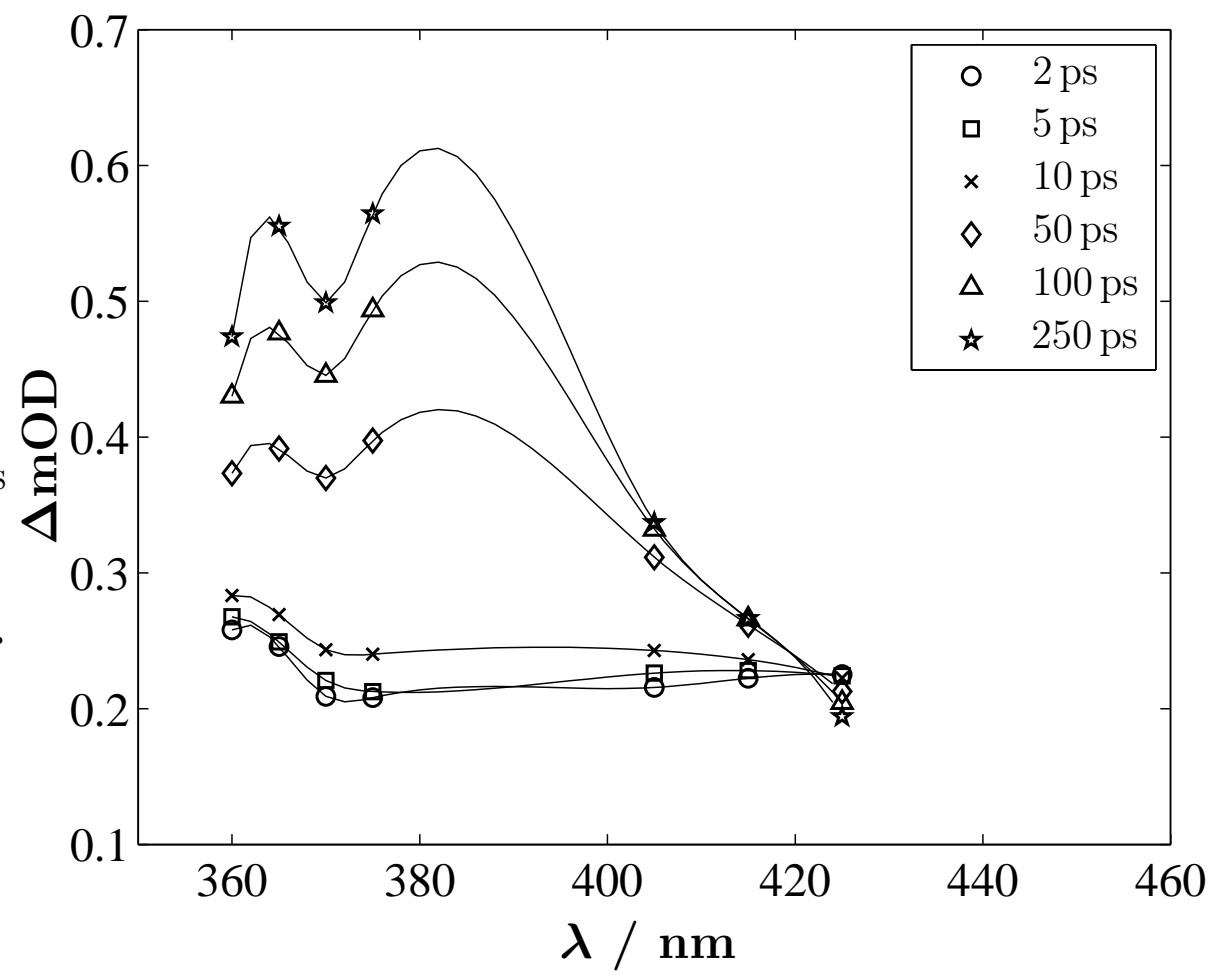

Abbildung 4.33: Transientes Absorptionsspektrum in überkritischem Xenon (308 K, 430 bar) bei $310 \mathrm{~nm}$ Anregung zu langen Zeiten.

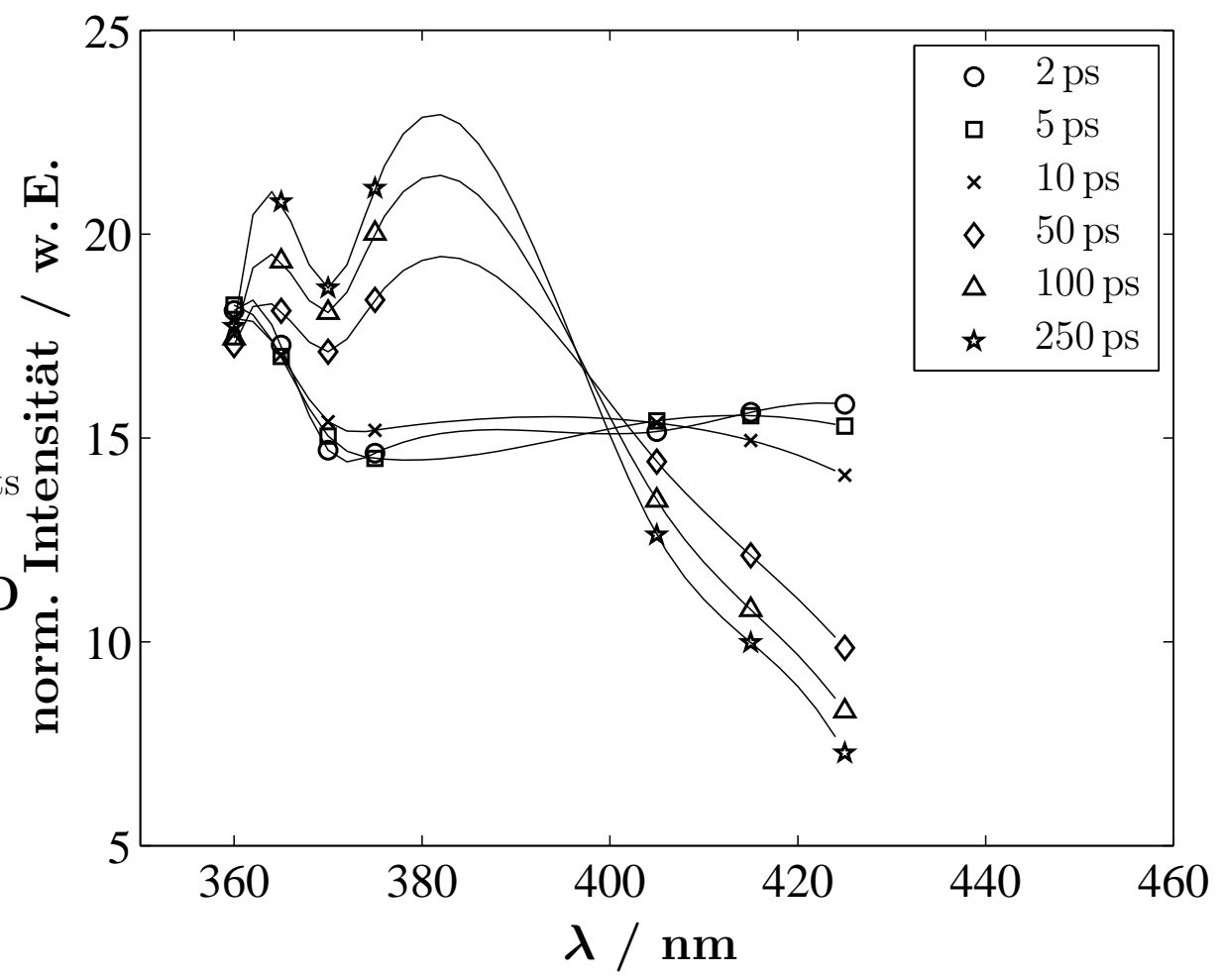

Abbildung 4.34: Auf Fläche normiertes, transientes Absorptionsspektrum in überkritischem Xenon (308 K, 430 bar) bei $310 \mathrm{~nm}$ Anregung zu langen Zeiten. 



\section{Kapitel 5}

\section{Diskussion}

In diesem Kapitel werden die im Vorangegangenen vorgestellten experimentellen Ergebnisse zur Konkurrenz zwischen der Photodissoziation und Photoisomerisierung in überkritischen Lösungsmitteln insbesondere in Hinblick auf die in Kapitel 1 angesprochenen Fragestellungen diskutiert.

\subsection{Beteiligte Spezies}

Zunächst soll geklärt werden, welche Spezies für die transienten Signale (siehe Kapitel 4 und Abbildung 5.1) im Kurzzeitbereich bis 2 ps nach der Photoanregung verantwortlich sind. Insbesondere wird der Bereich der isosbestischen Regionen in den normierten, transienten Spektren von $\sim 400 \mathrm{~nm}$ näher betrachtet.

Von vorneherein ist beispielsweise ein denkbarer Beitrag der Absorption von molekularem Iod zum transienten Signal bei $\sim 400 \mathrm{~nm}$ auszuschließen. Zunächst ist die Dissoziation des $\mathrm{CH}_{2} \mathrm{I}_{2}$ in $\mathrm{CH}_{2}$ und $\mathrm{I}_{2}$ aus Symmetriegründen im Anregungsbereich um $300 \mathrm{~nm}$ nicht möglich $[30,64,65]$ und kann daher auf einer kurzen Zeitskala nach Anregung nicht als direktes Dissoziationsprodukt gesehen werden. Eine schnelle homogene Iod/Iod-Rekombination und andere Effekte auf Grund von $\mathrm{CH}_{2} \mathrm{I}_{2}$-Clusterbildung scheiden wegen der verwendeten Konzentrationen an $\mathrm{CH}_{2} \mathrm{I}_{2}$ ebenfalls aus. Die Clusterbildung von mehreren $\mathrm{CH}_{2} \mathrm{I}_{2}$-Molekülen wurde von Åkesson et al. ebenfalls ausgeschlossen, da sie in ihren Messungen keine Konzentrationsabhängigkeit der transienten Signale nachweisen konnten [36]. Ferner ist eine signifikante Absorption des Iodmoleküls erst ab einer Wellenlänge $>420 \mathrm{~nm}$ festzustellen [150]. Die Absorption von später (nach einigen Nanosekunden) aus der homogenen Rekombination gebildetem, sich in der Messzelle anreicherndem, molekularem Iod kann aus diesem Grund ebenfalls vernachlässigt werden.

Im ersten schnellen transienten Absorptionsmaximum (siehe Abbildung 5.1) nach der Photoanregung kann ein Beitrag einer kohärenten Lösungsmittelantwort diskutiert 


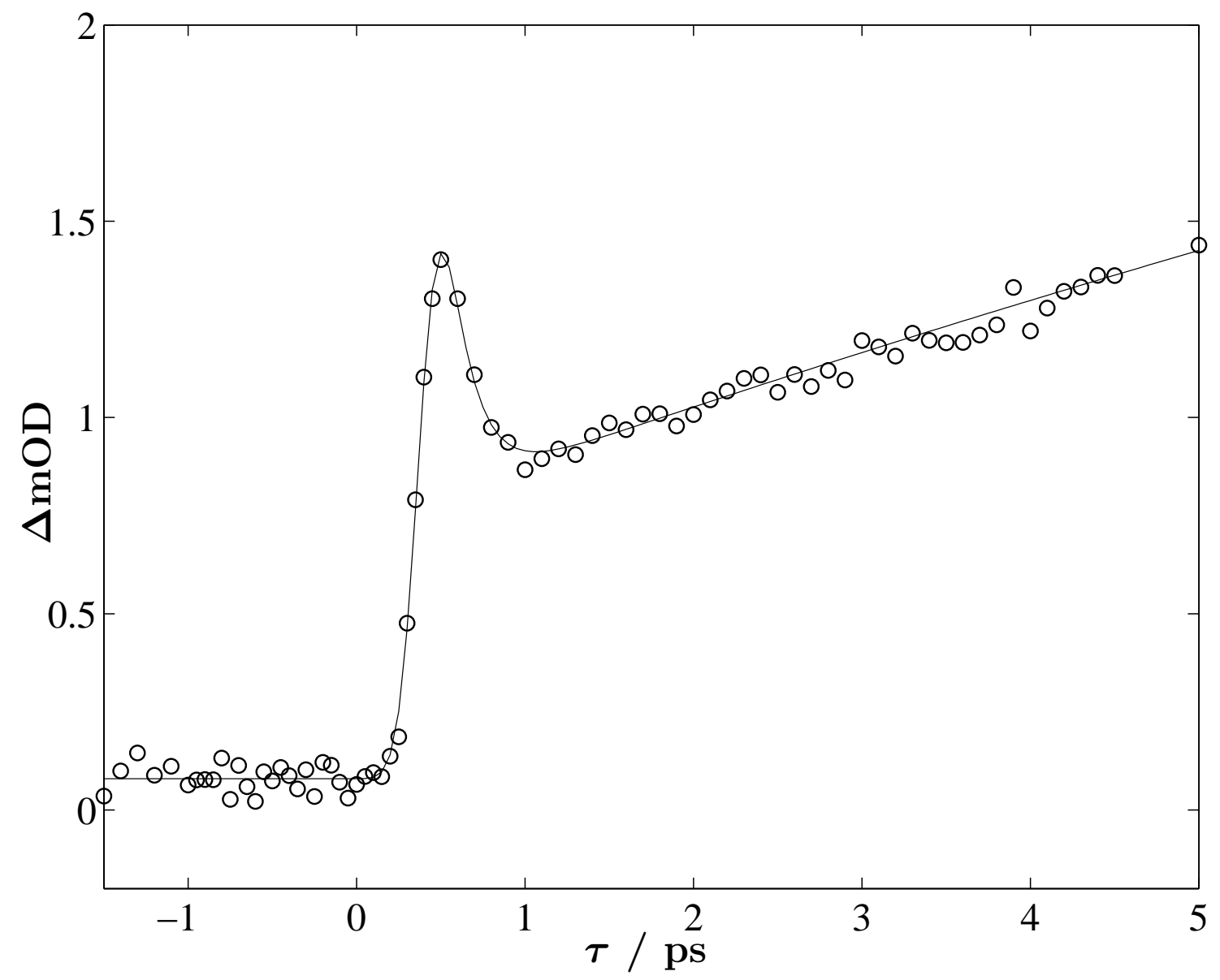

Abbildung 5.1: Typisches Signal im Bereich kurzer Verzögerungszeiten (überkritisches $\mathrm{CO}_{2} 308 \mathrm{~K}, 373$ bar, 304 nm Anregung / 370 nm Abfrage).

werden. Eine transiente Messung in reinem $\mathrm{CO}_{2}$ erbrachte eine nur sehr geringe, sich kaum vom Grundrauschen abhebende, Lösungsmittelantwort um den Zeitnullpunkt. Für die Lösungsmittel $\mathrm{CHF}_{3}$ und Ethan lassen sich ähnliche Beiträge erwarten. Lediglich in überkritischem Xenon ist ein höherer Beitrag der Lösungsmittelantwort möglich. Wie in ersten Untersuchungen bei einer Anregungswellenlänge von $280 \mathrm{~nm}$ und einer Abfragewellenlänge von 600-700 nm gezeigt werden konnte, liefert Xenon in diesem Fall eine kohärente Antwortfunktion mit einer Signalhöhe von bis zu 0,6 $\Delta \mathrm{mOD}$ und einer zeitlichen Breite von etwa 1 ps (siehe Abbildung 5.2). Dieses Verhalten ist evtl. auf Mehrfachionisationen des Xenons und Population von Rybergzuständen durch die hohen Spitzenintensitäten bei Femtosekunden Laserpulsen zu erklären [151,152,153]. Bei denen im Experiment verwendeten Anregungs- und Abfragewellenlängen konnte bislang nur eine sehr schwache, innerhalb des Grundrauschens liegende, derartige Antwort festgestellt werden. In Anbetracht der Tatsache, dass sich die Kohärenzen 


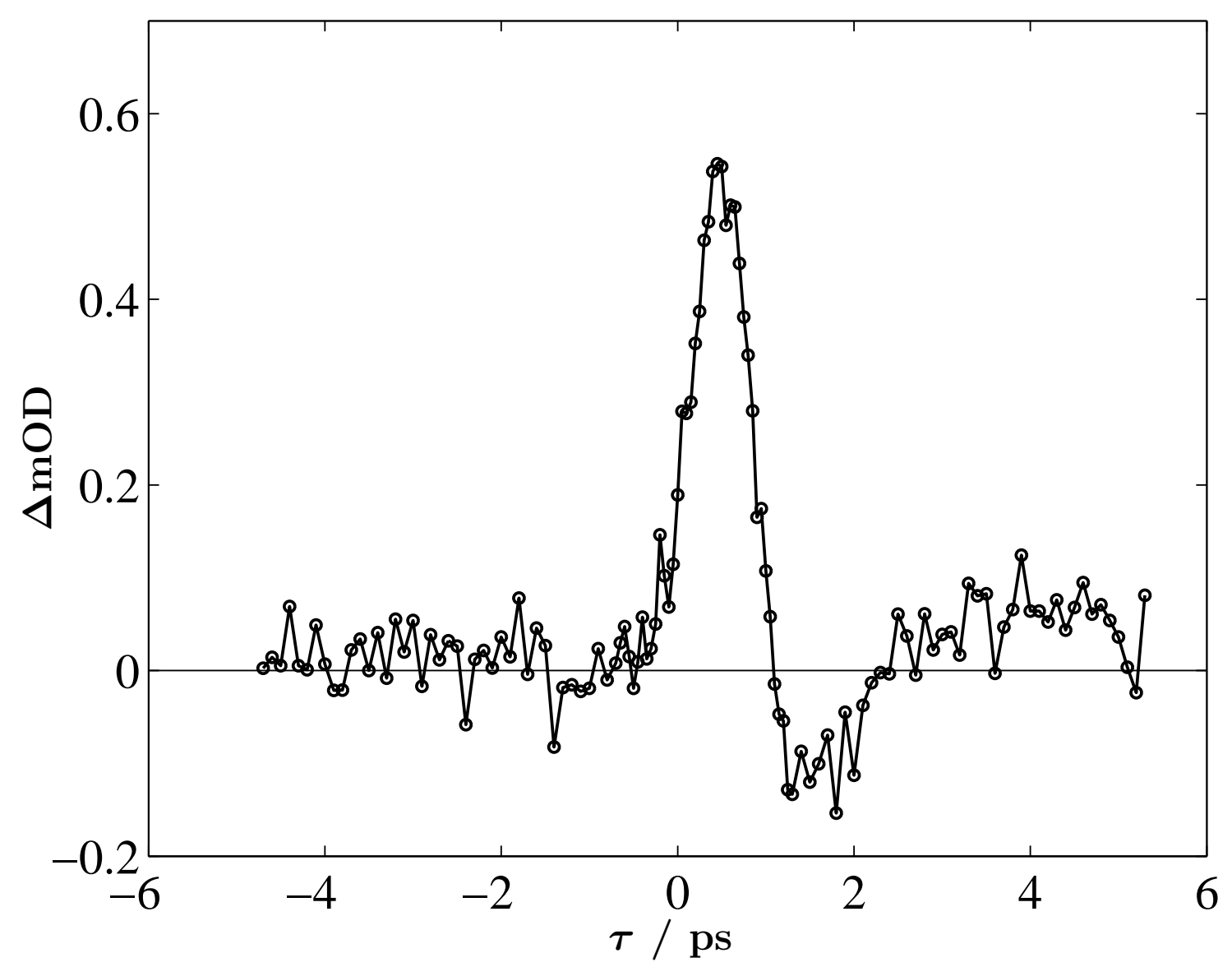

Abbildung 5.2: Kohärente Lösungsmittelantwort in überkritischem Xenon (308 K, 400 bar) bei $280 \mathrm{~nm}$ Anregung und $640 \mathrm{~nm}$ Abfrage.

nicht wesentlich aus dem Grundrauschen abheben, kann eine maximale Obergrenze von 10-15\% Beitrag zum ersten Absorptionsmaximum angenommen werden.

Folgende Spezies, deren Bedeutung anschließend diskutiert wird, könnten bei einer Abfragewellenlänge von $400 \mathrm{~nm}$ im Kurzzeitbereich bis zu 2 ps zur Dynamik beitragen:

1. Elektronisch angeregtes $\mathrm{CH}_{2} \mathrm{I}_{2}$-Molekül innerhalb der Franck-Condon-Region, wie von Ảkesson und Mitarbeitern diskutiert [36].

2. Das geminal, nach nur einem einzigen Stoß mit der Käfigwand, rekombinierte $\mathrm{CH}_{2} \mathrm{I}_{2}$ wie von Harris und Mitarbeitern vorgeschlagen [14].

3. Das $\mathrm{CH}_{2} \mathrm{I}-\mathrm{I}$-Isomer als sehr schnell gebildetes Käfigphotoprodukt [36]. 
4. Das direkt aus der Photodissoziation gebildete $\mathrm{CH}_{2} \mathrm{I}$-Radikal, wie u. a. von Yoshihara und Mitarbeitern vorgeschlagen [35].

5. Ein $\mathrm{CH}_{2} \mathrm{I} \cdots$ I-Kontakt-Ladungstransferkomplex (CCT) [154].

Die Möglichkeit des Beitrages der genannten Spezies soll im Folgenden genauer diskutiert werden.

\subsubsection{Franck-Condon-Region des angeregten $\mathrm{CH}_{2} \mathrm{I}_{2}$}

In der Vorstellung von Ảkesson et al. befindet sich das nach der Photoanregung auf der Potentialfläche des ersten elektronisch angeregten Zustands erzeugte Wellenpaket in einem Bereich in dem der Franck-Condon-Faktor groß genug ist um eine transiente Absorption in einen noch höheren elektronischen Zustand zu ermöglichen. Daher bildet sich das erste Absorptionsmaximum der Pump/Probesignale innerhalb der Zeitauflösung des Experimentes aus. Für die Gasphase wurden, wie bereits in Kapitel 2 beschrieben, zahlreiche Modelle für die Bewegung des gebildeten Wellenpaketes aufgestellt. Nach diesen Modellen wird meist von einem schnellen Verlassen des Wellenpaketes der ursprünglichen Franck-Condon-Region auf einer Femtosekundenzeitskala ausgegangen $[61,75]$ (siehe auch Kapitel 2). Dieses lässt den Schluss zu, dass es sich bei der ersten schnell abfallenden Komponente $\tau_{\text {Abfall }}$ in den transienten Absorptionszeitprofilen um das Verlassen des Wellenpaketes aus dem Franck-Condon-aktiven Bereich der Potentialfläche des ersten angeregten Zustandes handelt.

Für diese schnelle Komponente wurde in $\mathrm{CO}_{2}$ bei $370 \mathrm{~nm}$ Abfrage eine Dichteabhängigkeit gefunden. Dieses lässt die Vermutung zu, dass es sich um einen viskositätsabhängigen Reibungseinfluss auf die Bewegung auf der repulsiven Potentialfläche handelt, die zur Dissoziation führt. In einer Auftragung der Zeitkonstanten für den schnellen Abfall $\tau_{\text {Abfall }}$ des Signals in Abhängigkeit von der Lösungsmittelviskosität findet sich innerhalb der experimentellen Unsicherheiten ein linearer Verlauf (siehe Abbildung 5.3; die Umrechnung der Druck- in eine Dichteskala ist in Anhang A.3.1 beschrieben). Dieses Verhalten wurde schon für andere Photoisomerisierungen erhalten [155] und ist analog zur Iod-Dissoziation in komprimierter Lösung, die von Schroeder und Mitarbeitern untersucht wurde. Danach gibt es auch einen Einfluss der Viskosität des Lösungsmittels über die Reibung auf die Zeitkonstante der Dissoziation [12]. Dabei wurde das Modell eines über Stokes-Reibung gedämpften Harmonischen Oszillators zur Modellierung des Reibungseinflusses auf eine repulsive Potentialfläche verwendet.

Der Reibungseinfluss des Lösungsmittels auf die Dissoziationsgeschwindigkeit ist wahrscheinlich deshalb so gut sichtbar, weil der Großteil der zur Verfügung stehenden Energie nach der Dissoziation in die inneren Freiheitsgrade des $\mathrm{CH}_{2}$ I-Radikals übergeht und die kinetische Energie $\left(E_{\mathrm{kin}} \simeq 3000 \mathrm{~cm}^{-1}\right.$ bei $304 \mathrm{~nm}$ Anregung [73]) der Translationsbewegung nur einen geringen Teil ausmacht [56,73]. 


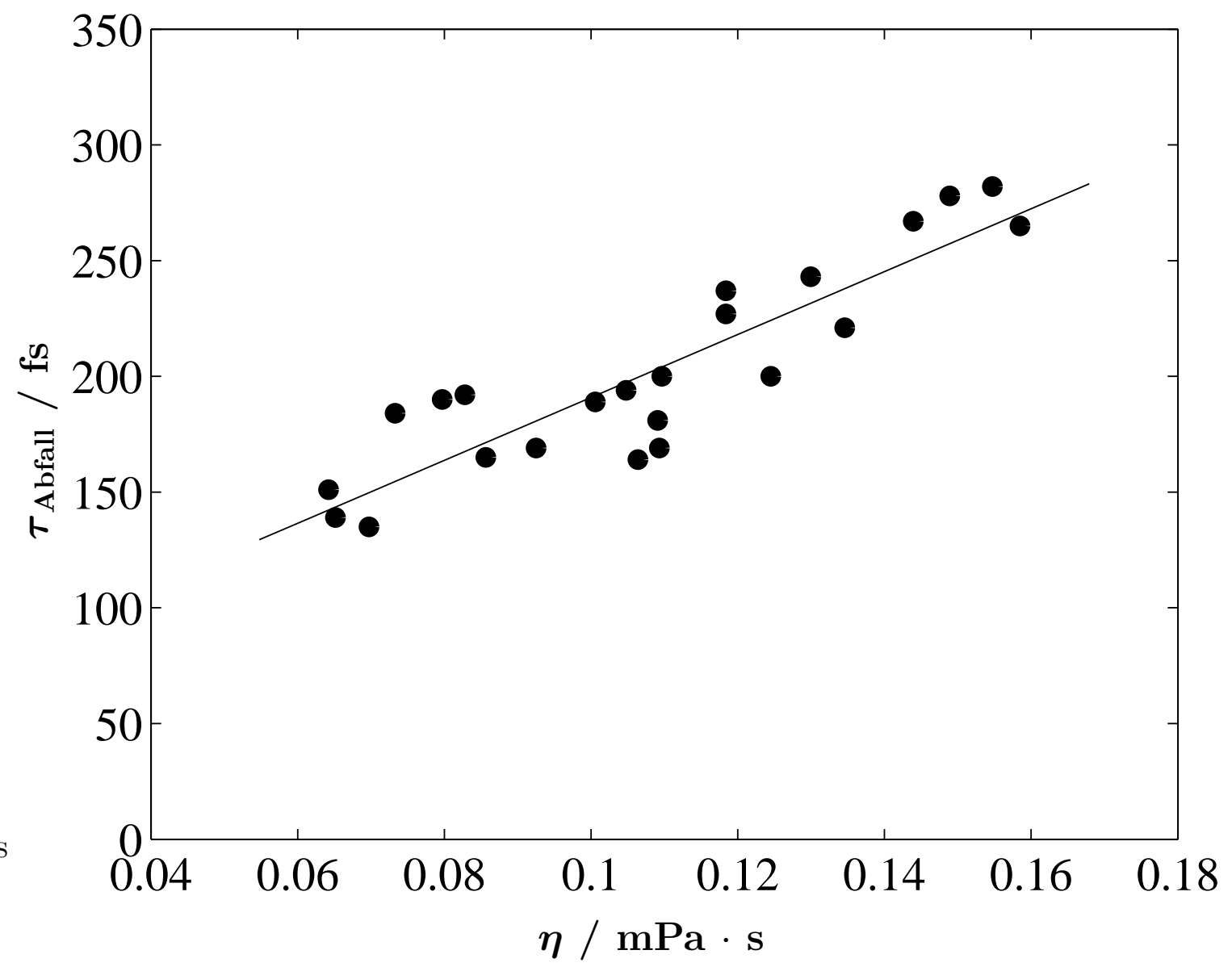

Abbildung 5.3: Viskositätsabhängigkeit der Zeitkonstanten des schnellen Abfalls $\tau_{\text {Abfall }}$ in überkritischem $\mathrm{CO}_{2}(308 \mathrm{~K})$ bei $304 \mathrm{~nm}$ Anregung und $370 \mathrm{~nm} \mathrm{Ab-}$ frage.

Die transiente Absorption des $\mathrm{CH}_{2} \mathrm{I}_{2}$ kann allerdings nicht die einzige Spezies sein, die im Kurzzeitbereich zur Signalbildung beiträgt, da eine Extrapolation der ansteigenden Komponente (Bildung des Isomers) zum Zeitnullpunkt, nicht bei Null beginnt. Auch an der relativen Höhe des Minimums der Kurve (siehe Abbildung 5.1) ist schon rein qualitativ abzulesen, dass weitere Spezies im Kurzzeitbereich eine nicht zu vernachlässigende Absorbanz aufweisen. Die Herkunft dieser Absorptionsbeiträge wird im Folgenden diskutiert. 


\subsubsection{Geminale Rekombination}

Um den möglichen Beitrag der geminalen Rekombination zum transienten Kurzzeitsignal zu diskutieren, wird zunächst eine Abschätzung der Energie, die den Fragmenten nach der Photodissoziation zur Verfügung steht, vorgenommen.

Wie bereits in Abschnitt 2.1 beschrieben, kann die verfügbare Energie $E_{\mathrm{v}}$ wie folgt abgeschätzt werden [56]:

$$
E_{\mathrm{v}}=h \nu-D_{0}^{0}+E_{\mathrm{in}}^{0},
$$

wobei die Differenz aus Dissoziationsenergie $D_{0}^{0}$ und innerer Energie $E_{\text {in }}^{0}$ über die Standardbildungsenthalpien gemäß $D_{0}^{0}-E_{\mathrm{in}}^{0} \simeq \Delta H_{298 \mathrm{~K}}^{\mathrm{f} \ominus}$ abgeschätzt werden kann. Die Enthalpie des Dissoziationsprozesses kann nach dem Satz von Hess aus den Standardbildungsenthalpien der beteiligten Spezies berechnet werden. Die Standardbildungsenthalpien bei $298 \mathrm{~K}$ wurden zu $217,6 \mathrm{~kJ} / \mathrm{mol}$ für $\mathrm{CH}_{2} \mathrm{I}$ [156], 106,8 kJ/mol für $\mathrm{I}\left({ }^{2} \mathrm{P}_{3 / 2}\right)$ [157] und $118,4 \mathrm{~kJ} / \mathrm{mol}$ für $\mathrm{CH}_{2} \mathrm{I}_{2}$ [157] bestimmt. Weil bei einer Anregungsenergie von $304 \mathrm{~nm}$ nur etwa $10 \%$ der entstehenden Iodatome im angeregten $\mathrm{I}^{*}\left({ }^{2} \mathrm{P}_{1 / 2}\right)$ vorliegen [62], kann in erster Näherung für diese Energiebetrachtung die Bildungsenthalpie des Iod im Grundzustand verwendet werden. Danach ergibt sich eine verfügbare Energie der Fragmente von $188 \mathrm{~kJ} / \mathrm{mol}\left(\sim 15700 \mathrm{~cm}^{-1}\right)$. Unter der Voraussetzung, das etwa $80 \%$ der verfügbaren Energie als innere Energie des $\mathrm{CH}_{2} \mathrm{I}$-Fragmentes vorliegt, besitzt das $\mathrm{CH}_{2} \mathrm{I}$-Radikal direkt nach der Photodissoziation eine innere Energie von etwa $150 \mathrm{~kJ} / \mathrm{mol}\left(\sim 12500 \mathrm{~cm}^{-1}\right)$, während die restliche Energie in die kinetische Energie der Translation der Fragmente übergeht. Daraus ergeben sich folgende Argumente, die gegen einen Beitrag der geminalen Rekombination sprechen:

- Falls eine Rekombinationsreaktion zum $\mathrm{CH}_{2} \mathrm{I}_{2}$ aus einem solch stark schwingungsheißen Radikalfragment stattfindet, muss das Rekombinationsprodukt im Grundzustand stabilisiert werden. Die Schwingungsenergietransferzeit z. B. in überkritischem $\mathrm{CO}_{2}$ wurde jedoch auf einige zehn Pikosekunden bei einer Überschussenergie von etwa $6000 \mathrm{~cm}^{-1}$ [89] bestimmt und wahrscheinlich wird sich diese Zeit nicht um 1-2 Größenordnungen bei einer höheren Anregung ändern. Es könnte daher möglich sein, dass nicht genügend Schwingungsenergie auf einer Femtosekundenzeitskala ins Lösungsmittel transferiert werden kann, um das rekombinierte $\mathrm{CH}_{2} \mathrm{I}_{2}$ zu stabilisieren.

- Ein weiterer Aspekt, der gegen eine schnelle Rekombination auf einer Femtosekundenzeitskala spricht, ist die Tatsache, dass auf Grund der schnellen Rotation des $\mathrm{CH}_{2} \mathrm{I}$ die aus der Dissoziation entstandenen Fragmente schon nach sehr kurzer Zeit sterisch sehr ungünstig für eine geminale Rekombination stehen.

- Falls das rekombinierte, dann sehr schwingungsheiße, $\mathrm{CH}_{2} \mathrm{I}_{2}$ entstehen würde, müsste die daraus resultierende breite Absorptionsbande im Bereich von 300 $400 \mathrm{~nm}$ in den transienten Messungen sichtbar sein und ebenfalls auf Grund des 


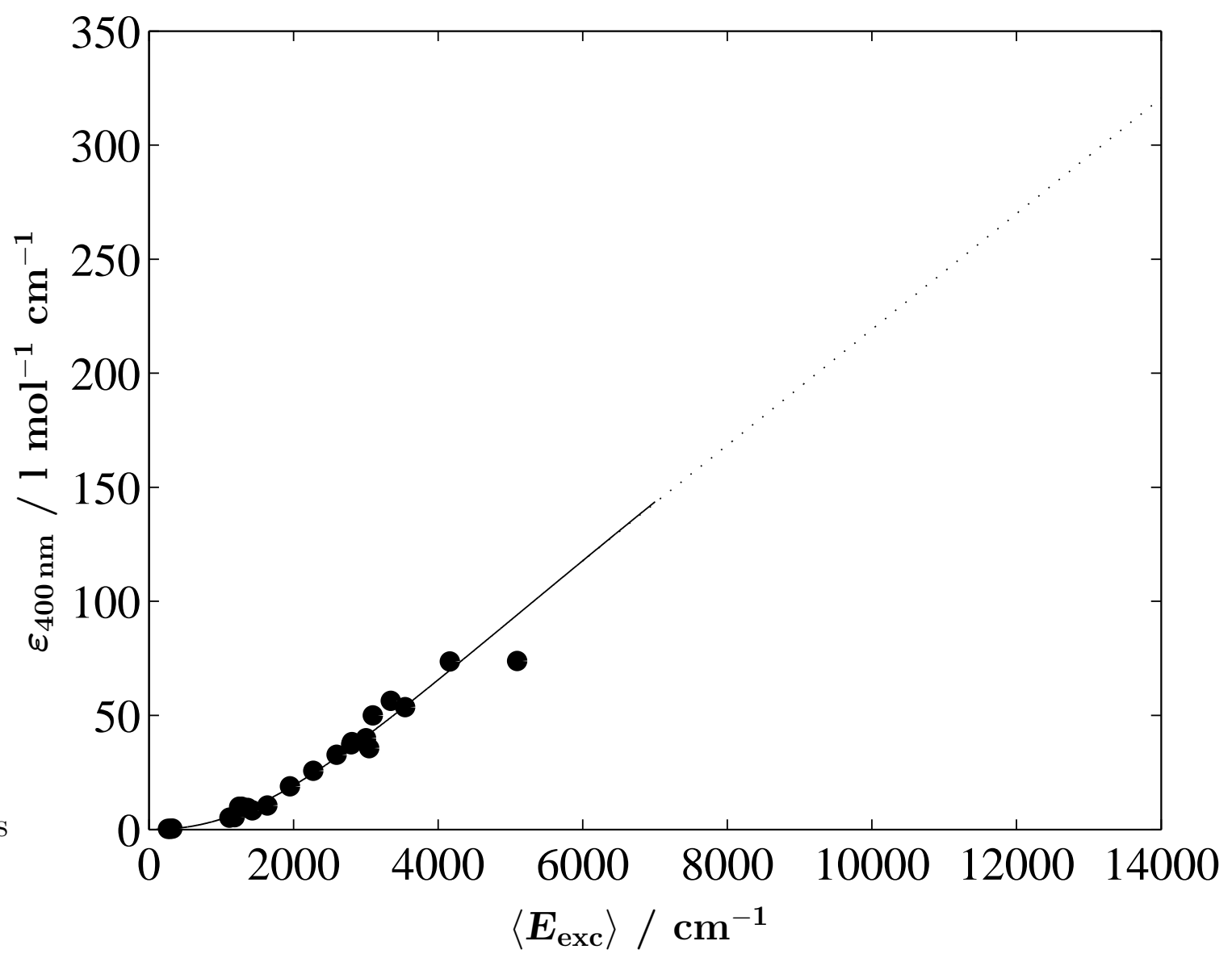

Abbildung 5.4: Extrapolation der aus Stoßwellendaten bestimmten Korrelation des Extinktionskoeffizienten $\varepsilon_{400 \mathrm{~nm}}$ mit der Überschussenergie des $\mathrm{CH}_{2} \mathrm{I}_{2}$ [88].

relativ langsamen Energietransfers auf einer Pikosekundenzeitskala sichtbar sein. Der mögliche Extinktionskoeffizient des schwingungsheißen $\mathrm{CH}_{2} \mathrm{I}_{2}$ kann aus einer linearen Extrapolation einer, aus Stoßwellendaten bestimmten, Korrelation des Extinktionskoeffizienten mit der inneren Energie bestimmt werden (siehe Abbildung 5.4) [88]. Bei einer derart hohen Überschussenergie im Bereich von etwa $12000 \mathrm{~cm}^{-1}$ liegt demnach der Extinktionskoeffizient bei $250-300 \mathrm{lmol}^{-1} \mathrm{~cm}^{-1}$, so dass die Änderung der transienten Absorption bei Auftreten der Spezies deutlich sichtbar sein sollte. Jedoch wurden weder von Åkesson et al. in den transienten Absorptionsmessungen im Bereich des Maximums der Absorptionsbande in flüssiger Lösung noch in den in dieser Arbeit gezeigten Messungen in überkritischen Lösungsmitteln Anzeichen für ein Ausbleichen des transienten Signals durch die Absorption von schwingungsangeregtem, rekombiniertem $\mathrm{CH}_{2} \mathrm{I}_{2}$ gefunden. 
Es ist daher ein Beitrag geminaler Rekombination zum $\mathrm{CH}_{2} \mathrm{I}_{2}$ bei den genannten experimentellen Bedingungen auszuschließen.

\subsubsection{Schnelle Isomerbildung}

Sowohl Åkesson und Mitarbeiter [36] als auch die Gruppe von Phillips [37] diskutieren die Bildung des schwingungsheißen $\mathrm{CH}_{2} \mathrm{I}$-I-Photoprodukts durch einen schnellen Käfigprozess innerhalb einer sehr kurzen Zeitspanne nach Anregung. Dies begründeten sie mit der Ähnlichkeit des transienten Spektrums nach nur einer Pikosekunde mit dem Langzeitproduktspektrum.

Die sehr schnelle Bildung des Isomers nach nur wenigen Stößen mit dem Lösungsmittelkäfig erscheint aus mehreren Gründen nicht sehr wahrscheinlich. Zum einen wurde die Dissoziationsenthalpie für das $\mathrm{CH}_{2} \mathrm{I}-\mathrm{I}$ zu $\mathrm{CH}_{2} \mathrm{I}$ und I mit ab initio Methoden von Glukhovtsev und Bach zu 19,4kJ/mol und die Barriere für die Rückisomerisierung von $\mathrm{CH}_{2} \mathrm{I}-\mathrm{I}$ zu $\mathrm{CH}_{2} \mathrm{I}_{2}$ zu $134 \mathrm{~kJ} / \mathrm{mol}$ berechnet [94]. Phillips et al. berechneten diese Barriere zu $60 \mathrm{~kJ} / \mathrm{mol}$ [100]. Der erhebliche Unterschied liegt wahrscheinlich in der unterschiedlichen Wahl der quantenchemischen Methoden, so dass sich ein realistischer Wert vermutlich irgendwo zwischen diesen Werten findet. Alle genannten Werte für Dissoziationsenthalpie und Rückisomerisierungsbarriere sind jedoch alle kleiner als die anfangs in den Fragmenten verfügbare Überschussenergie, so dass davon ausgegangen werden kann, dass eine Stabilisierung des $\mathrm{CH}_{2} \mathrm{I}-\mathrm{I}$-Isomers auf einer sehr kurzen Zeitskala nach nur wenigen Stößen mit der Käfigwand nicht möglich sein wird. Zum anderen sollte die schnelle Bildung des $\mathrm{CH}_{2} \mathrm{I}$-I-Isomers, wenn möglich, stark von der Größe und der Steifheit des Lösungsmittelkäfigs abhängen. So wäre eine Abnahme der Ausbeute an direkt gebildetem Isomer und damit eine Abnahme der Höhe des Anfangssignals mit abnehmender Dichte zu vermuten, da der konkurrierende Kanal zum Verlassen des Käfigs durch die Fragmente mit abnehmender Dichte immer mehr an Bedeutung gewinnen sollte. Jedoch wurde ein solcher Einfluss der Dichte auf das Anfangssignal in den durchgeführten dichteabhängigen Messungen in überkritschen Lösungsmitteln nicht beobachtet.

\subsection{4 $\mathrm{CH}_{2} \mathrm{I}$-Radikalabsorption}

In Abschnitt 5.1.2 wurde bereits die im $\mathrm{CH}_{2} \mathrm{I}$ verfügbare Überschussenergie direkt nach der Dissoziation mit etwa $12500 \mathrm{~cm}^{-1}$ abgeschätzt. Weil Baughcum und Leon in IR-Fluoreszenzmessungen intensive Emissionen des $\mathrm{CH}_{2} \mathrm{I}$ über einen großen Wellenlängenbereich detektiert haben [71], kann daraus geschlossen werden, dass die Überschussenergie nahezu statistisch über die inneren Freiheitsgrade des Radikals verteilt ist. Dies führt zu der Annahme, dass die intramolekulare Energieumverteilung (IVR) 
schnell genug ist, um die Energie innerhalb einer kurzen Zeitspanne statistisch zu verteilen. Im Widerspruch zu dieser Annahme wurde die Zeitkonstante des IVR für das $\mathrm{CH}_{2} \mathrm{I}_{2}$ bei $6000 \mathrm{~cm}^{-1}$ Anregung von Kajimoto et al. [89] in überkritischem $\mathrm{CO}_{2} \mathrm{zu}$ etwa 20 ps bestimmt. Es ist jedoch zu vermuten, dass im $\mathrm{CH}_{2}$ I-Radikal durch den Bindungsbruch im Wesentlichen die C-I-Streckschwingung sehr hoch angeregt und durch Kopplungen der intramolekulare Energiefluss viel effektiver und schneller ist als unter den bei Kajimoto herrschenden experimentellen Bedingungen für das $\mathrm{CH}_{2} \mathrm{I}_{2}$.

Die mittlere innere Energie $\left\langle E_{\mathrm{CH}_{2} \mathrm{I}}\right\rangle$ des Radikals kann, unter der Annahme dass sie schnell auf die inneren Freiheitsgrade umverteilt ist, folgendermaßen beschrieben werden:

$$
\left\langle E_{\mathrm{CH}_{2} \mathrm{I}}\right\rangle=\sum_{i=1}^{6} \frac{h \nu_{i}}{\exp \left(\frac{h \nu_{i}}{k_{\mathrm{B}} T}\right)-1}+\frac{3}{2} k_{\mathrm{B}} T
$$

Dabei wird auf Grund der hohen Anregung für den rotatorischen Beitrag der Hochtemperaturgrenzwert $3 / 2 k_{\mathrm{B}} T$, und auf Grund des vorausgesetzten schnellen IVR gleiche Schwingungs- und Rotationstemperatur angenommen. Werden nun für die Schwingungen die von Phillips etal. [96] mit ab initio Methoden berechneten Schwingungsfrequenzen für das $\mathrm{CH}_{2}$ I-Radikal $\left(\nu_{1}=3174 \mathrm{~cm}^{-1}, \nu_{2}=1353 \mathrm{~cm}^{-1}\right.$, $\nu_{3}=609 \mathrm{~cm}^{-1}, \nu_{4}=166 \mathrm{~cm}^{-1}, \nu_{5}=3335 \mathrm{~cm}^{-1}, \nu_{6}=855 \mathrm{~cm}^{-1}$ ) einsetzt, wird eine Schwingungstemperatur von $T \simeq 3100 \mathrm{~K}$ erhalten. Bei einer derart hohen Schwingungstemperatur ist davon auszugehen, dass das UV-Absorptionsspektrum gegenüber dem thermischen Spektrum stark verbreitert ist [142]. Das thermische Absorptionsspektrum (siehe Abbildung 2.3 auf Seite 12) verfügt über zwei ausgeprägte Banden bei $280 \mathrm{~nm}$ und $340 \mathrm{~nm}$. Wird für das $\mathrm{CH}_{2}$ I-Radikal bei $400 \mathrm{~nm}$ ein ähnlicher Verlauf des Extinktionskoeffizienten mit der inneren Energie angenommen wie beim $\mathrm{CH}_{2} \mathrm{I}_{2}$, kann aus den relativen Höhen der Extinktionen an den Absorptionsmaxima von $\mathrm{CH}_{2} \mathrm{I}_{2}\left(\varepsilon_{\mathrm{CH}_{2} \mathrm{I}_{2}, 297 \mathrm{~nm}}=1100 \mathrm{lmol}^{-1} \mathrm{~cm}^{-1}\right.$ [62]) und des $\mathrm{CH}_{2}$ I-Radikals $\left(\varepsilon_{\mathrm{CH}_{2} \mathrm{I}, 340 \mathrm{~nm}}=1200 \mathrm{lmol}^{-1} \mathrm{~cm}^{-1}[74]\right)$ und den relativen spektralen Entfernungen $\mathrm{zu}$ $400 \mathrm{~nm}$ der Extinktionskoeffizient des schwingungsheißen $\mathrm{CH}_{2} \mathrm{I}$ grob abgeschätzt werden. Dazu wird die, bereits beschriebene (siehe Abschnitt 5.1.2), Korrelation der Überschussenergie mit der Extinktion verwendet, welche bei einer Überschussenergie von $12000 \mathrm{~cm}^{-1}$ im $\mathrm{CH}_{2} \mathrm{I}_{2}$ bei $400 \mathrm{~nm}$ einen Extinktionskoeffizienten von $\varepsilon_{\mathrm{CH}_{2} \mathrm{I}_{2}, 400 \mathrm{~nm}} \simeq 280 \mathrm{l} \mathrm{mol}^{-1} \mathrm{~cm}^{-1}$ liefert. Daraus ergibt sich nach der beschriebenen Abschätzung ein Extinktionskoeffizient von $\varepsilon_{\mathrm{CH}_{2} \mathrm{I}, 400 \mathrm{~nm}}>100 \mathrm{lmol}^{-1} \mathrm{~cm}^{-1}$. Hierbei ist $\mathrm{zu}-$ nächst die Möglichkeit noch außer Acht gelassen, dass die bei $280 \mathrm{~nm}$ zentrierte Radikalabsorptionsbande bei solch hohen inneren Energien einen Beitrag zur Absorption bei $400 \mathrm{~nm}$ leisten kann. Da das Radikal direkt schwingungsheiß aus der Dissoziation hervorgeht, sollte zu kurzen Verzögerungszeiten auf Grund dieser Abschätzungen ein Beitrag der Radikalabsorption vorhanden sein. Die Quantenausbeute an schwingungsheiß gebildetem $\mathrm{CH}_{2} \mathrm{I}$ wird nun auf Grund der Tatsache, dass das Vorhandensein von etwa $10 \%$ angeregten Iodatomen weniger Ausbeute an hoch schwingungsangeregtem 
Radikal bewirkt, zu 0,9 angenommen. Die Änderung der Absorption $\Delta A$ kann bei einem Extinktionskoeffizienten des Radikals von $100 \leq \varepsilon_{\mathrm{CH}_{2} \mathrm{I}, 400 \mathrm{~nm}} \leq 4001 \mathrm{~mol}^{-1} \mathrm{~cm}^{-1}$ auf $0,04 \leq \Delta A \leq 0,16 \Delta \mathrm{mOD}$ abgeschätzt werden. Dies würde etwa 10-40\% des ersten Absorptionsmaximums entsprechen. Dieses Signal wird allerdings auf einer kurzen Zeitskala wieder abklingen, da der Schwingungsenergietransfer in das Lösungsmittel zu einem schnellen Verschwinden der Absorptionsflanke bei 400 nm führt. Die Schwingungsenergierelaxationszeiten von $\mathrm{CH}_{2} \mathrm{I}_{2}$ in überkritischem $\mathrm{CO}_{2}$ wurden von Kajimoto [89] zwar im relevanten Dichtebereich zu 50-80 ps bestimmt, jedoch wurde von Baughcum und Leon eine um eine Größenordnung schnellere Schwingungsrelaxation für das $\mathrm{CH}_{2} \mathrm{I}$ angenommen als für das $\mathrm{CH}_{2} \mathrm{I}_{2}$ [71], so dass die heiße Absorption des Radikals in diesem Spektralbereich innerhalb weniger Pikosekunden abklingen sollte.

Im Bereich kürzerer Abfragewellenlängen müsste demnach ein noch stärkerer Beitrag der Radikalabsorption sichtbar sein. Åkesson und Mitarbeiter konnten in ihren Spektren allerdings keine transiente Absorption im Bereich $<300 \mathrm{~nm}$ feststellen. Jedoch ist der Schwingungsenergietransfer in Acetonitril, welches von Åkesson et al. verwendet wurde, eventuell auf Grund der Polarität des Lösungsmittels [158] oder der besseren Kopplung der Solvat- an die Solventmoden sehr viel schneller. Breitbandige transiente Kurzzeitspektren im Bereich von 280-400 nm in anderen evtl. unpolaren Lösungsmitteln mit langsamen VET, wie z. B. $\mathrm{CCl}_{4}$ sind bislang zum Vergleich aus der Literatur nicht verfügbar. Die in dieser Arbeit vorgestellten Spektren können auf Grund der spektralen Limitierung leider ebenfalls nicht zum Vergleich herangezogen werden.

\subsubsection{Orientierungsrelaxation}

Einen weiteren Hinweis auf die am Kurzzeitsignal beteiligten Spezies geben die dichteabhängigen Anisotropiemessungen in überkritischem $\mathrm{CO}_{2}$ (siehe Abbildung 4.20 auf Seite 71). Gemäß der bisher geführten Diskussion zu den beitragenden Spezies kommen für das Signal das elektronisch angeregte $\mathrm{CH}_{2} \mathrm{I}_{2}$, das $\mathrm{CH}_{2}$ I-Radikal, das $\mathrm{CH}_{2} \mathrm{I}$-I-Isomer und das CCT-Komplexpaar aus $\mathrm{CH}_{2} \mathrm{I}$ und I (siehe Abschnitt 5.1.6) für den Ursprung der Anisotropie in Frage. Sollte die Anisotropie nur von einer Spezies herrühren, würde theoretisch, je nach Molekülgeometrie, ein triexponentiell abklingender Verlauf mit bis zu drei Rotationsrelaxationszeiten zu erwarten sein [159], jedoch ist eine Trennung der Zeitkonstanten im Allgemeinen experimentell nicht möglich [160,161].

Um die Anisotropie verursachende Spezies zu identifizieren, wird zunächst die Viskositätsabhängigkeit der aus den Anisotropien ermittelten Rotationsrelaxationszeiten $\tau_{\text {rot }}$ untersucht. Die Rotationsrelaxationszeiten werden mit abnehmender Viskosität schneller (siehe Abbildung 5.5).

Diese starke Lösungsmittelviskositätsabhängigkeit der Orientierungsrelaxation deutet auf eine große Solvat-Solventwechselwirkung der rotierenden Spezies hin, die die Ori- 


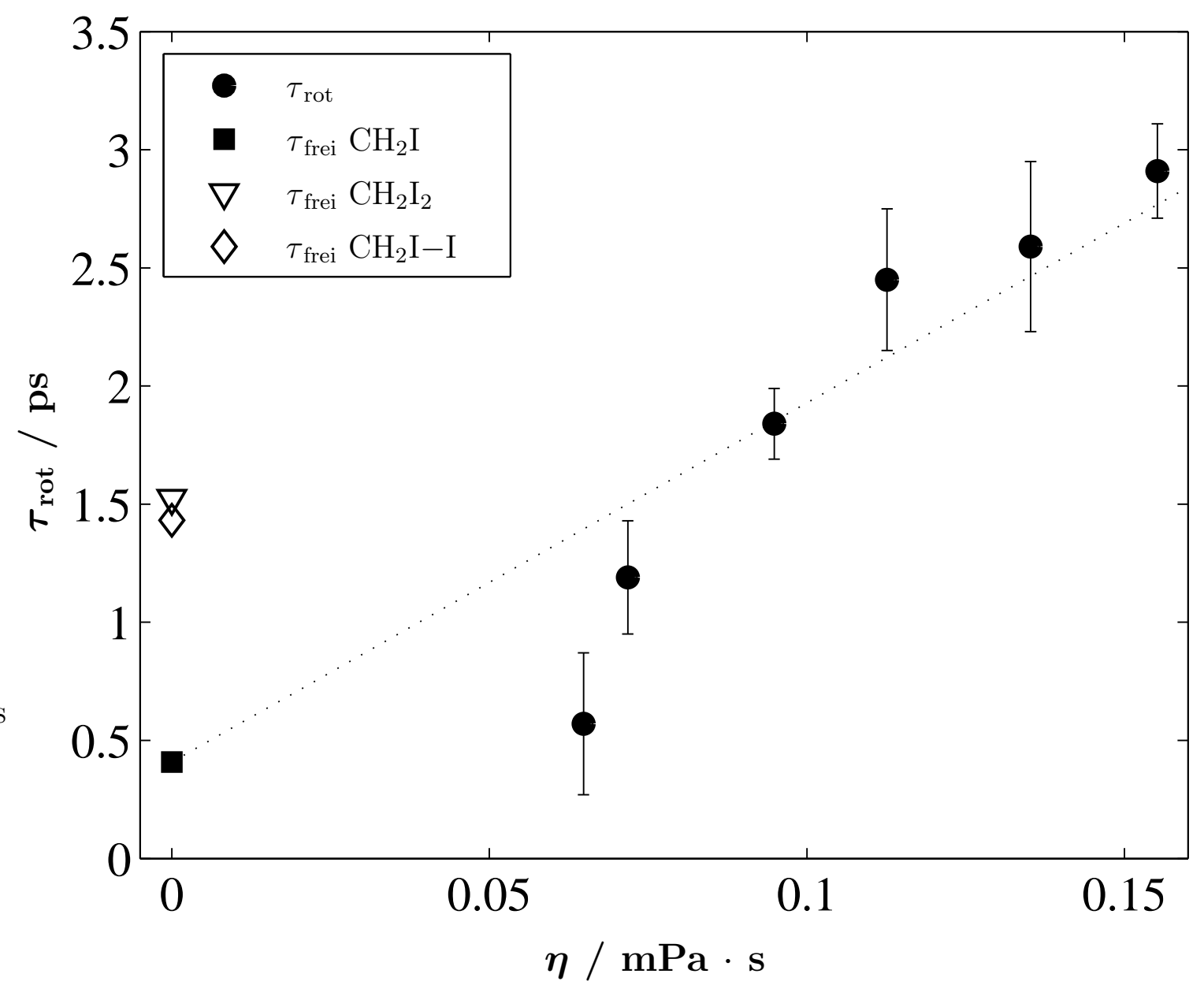

Abbildung 5.5: Viskositätsabhängigkeit der Rotationsrelaxationszeiten $\tau_{\text {rot }}$ aus Anisotropiemessungen in überkritischem $\mathrm{CO}_{2}(308 \mathrm{~K})$ bei $305 \mathrm{~nm}$ Anregung und $405 \mathrm{~nm}$ Abfrage und Vergleich mit den freien Rotorzeiten $\tau_{\text {frei }}$ der in Frage kommenden Spezies.

entierungsrelaxation hindert. Weil diese Abhängigkeit der Orientierungsrelaxationszeit von der Viskosität um fast eine Größenordnung stärker ist als in anderen Halogenmethanen, wie zum Beispiel bei flüssigem $\mathrm{CHBr}_{3}$ [162] oder bei überkritischem $\mathrm{CHF}_{3}$ [163], kann vermutet werden, dass es sich um das Radikal handelt.

Um diese Vermutung zu bestätigen, können die berechneten freien Rotorzeiten der Spezies mit den experimentellen Daten verglichen werden. Für den Einfluss der Viskosität $\eta$ auf die Rotationsrelaxationszeit $\tau_{\text {rot }}$ gilt nach dem Stokes-Einstein-Debye-Modell folgende lineare Beziehung [164, 165]:

$$
\tau_{\text {rot }}=\tau_{0}+\frac{\eta V f}{6 k_{\mathrm{B}} T}
$$


Dabei bezeichnet $V$ das hydrodynamische Volumen und $f$ einen molekularen Formfaktor, der Werte zwischen 0 und 1 annehmen kann $[166,167]$. Der Achsenabschnitt $\tau_{0}$ kann nun mit der freien Rotorzeit gleichgesetzt werden, $\tau_{0}=\tau_{\text {frei }}[168]$. Für die freie Rotorzeit gilt folgende Beziehung:

$$
\tau_{\text {frei }}=\left(\frac{4 \pi}{3}\right)^{\frac{1}{2}} \omega_{0}^{-1},
$$

mit der Rotationsfrequenz $\omega_{0}$ des freien Rotors. Daraus folgt mit

$$
\omega_{0}=\left(\frac{6 k_{\mathrm{B}} T}{\Theta}\right)^{\frac{1}{2}}
$$

und dem molekularen Hauptträgheitsmoment $\Theta$ für die freie Rotorzeit:

$$
\tau_{\text {frei }}=\left(\frac{2 \pi \Theta}{9 k_{\mathrm{B}} T}\right)^{\frac{1}{2}} .
$$

Für den Zusammenhang zwischen Hauptträgheitsmoment und der Rotationskonstante um eine Hauptträgheitsachse gilt wiederum:

$$
\Theta=\frac{h}{8 \pi^{2} A},
$$

wobei $A$ für eine beliebige Rotationskonstante steht. Danach kann die Beziehung für die freie Rotorzeit in Abhängigkeit von der Rotationskonstanten geschrieben werden als:

$$
\tau_{\text {frei }}=\frac{1}{6}\left(\frac{h}{\pi k_{\mathrm{B}} T A}\right)^{\frac{1}{2}} .
$$

Eigentlich würde sich die Rotationsrelaxationszeit aus einem gewichteten Mittel der freien Rotorzeiten für jede Rotationskonstante zusammensetzen, jedoch kommt es für die Gewichtung im Wesentlichen auf die relative Lage des Übergangsdipolmomentes zur entsprechenden Hauptträgheitsachse an [160]. Für die Spezies $\mathrm{CH}_{2} \mathrm{I}_{2}$ und $\mathrm{CH}_{2} \mathrm{I}-\mathrm{I}$ ist die Lage des Übergangsdipolmomentes wahrscheinlich senkrecht zur I-IVerbindungslinie und bei $\mathrm{CH}_{2} \mathrm{I}$ senkrecht zur C-I-Verbindungslinie. Die I-I- bzw. C-IVerbindungslinie sind aber auf Grund der hohen Iodmassen im Vergleich zu den C und H Massen und der Molekülgeometrie nahezu parallel mit der Hauptträgheitsachse des Moleküls, die die größte Rotationskonstante hat. So finden sich für alle Spezies zwei sehr dicht aneinander liegende Hauptträgheitsmomente und jeweils ein um mehr als eine Größenordnung kleineres Trägheitsmoment.

Für die Berechnung der freien Rotorzeiten der Spezies wurde demnach, in erster Näherung, das geometrische Mittel der beiden größeren Trägheitsmomente verwendet. Bei 
$\mathrm{CH}_{2} \mathrm{I}_{2}$ wurden für die genaue Berechnung der Rotationszeit die von Kisiel et al. experimentell bestimmten Rotationskonstanten verwendet [59], um die Trägheitsmomente nach Gleichung $5.7 \mathrm{zu}$ berechnen. Bei $\mathrm{CH}_{2} \mathrm{I}$ wurden die mit ab initio Methoden von Seetula berechneten Trägheitsmomente [169] und bei $\mathrm{CH}_{2} \mathrm{I}-\mathrm{I}$ die von Phillips et al. berechneten Molekülgeometrien verwendet. Für die Berechnung der Trägheitsmomente aus den Molekülgeometrie im Schwerpunksystem gilt:

$$
\Theta=\sum_{i} m_{i} r_{i}^{2}
$$

wobei $m_{i}$ die Masse und $r_{i}$ der Abstand des $i$ ten Atoms von der Achse ist. Für die Spezies werden aus den gemittelten Trägheitsmomenten $\Theta_{\mathrm{CH}_{2} \mathrm{I}_{2}}=1,397 \cdot 10^{-44} \mathrm{~kg} \mathrm{~m}{ }^{2}$, $\Theta_{\mathrm{CH}_{2} \mathrm{I}-\mathrm{I}}=1,15 \cdot 10^{-44} \mathrm{~kg} \mathrm{~m}^{2}$ und $\Theta_{\mathrm{CH}_{2} \mathrm{I}}=0,096 \cdot 10^{-44} \mathrm{~kg} \mathrm{~m}^{2}$ die mittleren freien Rotorzeiten $\mathrm{zu} \tau_{\mathrm{CH}_{2} \mathrm{I}_{2}}=1,52 \mathrm{ps}, \tau_{\mathrm{CH}_{2} \mathrm{I}-\mathrm{I}}=1,40 \mathrm{ps}$ und $\tau_{\mathrm{CH}_{2} \mathrm{I}_{2}}=0,41 \mathrm{ps}$ bestimmt. Bei einer Extrapolation der gemessenen Rotationsrelaxationszeiten zu $\eta=0$ zeigt sich, dass der Wert für die freie Rotorzeit des $\mathrm{CH}_{2}$ I-Radikals am Besten mit der Extrapolation übereinstimmt. Dies kann als weiteres Indiz für einen Beitrag der Radikalspezies zum transienten Kurzzeitsignal gedeutet werden.

\subsubsection{Ladungstransferkomplex}

Weil das $\mathrm{CH}_{2} \mathrm{I}$-I-Isomer nicht als beitragende Spezies im Kurzzeitbereich in Frage kommt, wurde als Ursache für die sehr schnell nach der Anregung auftretende Absorption im Bereich von $355 \mathrm{~nm}$ ein schwach gebundener, so genannter KontaktLadungstransferkomplexes (CCT, contact charge transfer) [170,171] als transient absorbierende Spezies postuliert [154]. Die Bildung eines Ladungstransferkomplexes (CT, charge transfer) aus $\mathrm{CH}_{2} \mathrm{I}$ und I wurde als mögliches Photoprodukt der Photodissoziation von $\mathrm{CH}_{2} \mathrm{I}_{2}$ in Lösung bereits diskutiert [35, 85], jedoch wurde dabei der langsame ps-Anstieg des Signals der Bildung eines solchen Ladungstransferkomplexes zugeschrieben [35]. Ein schwach gebundener CCT-Komplex wurde auch schon von Phillips und Mitarbeitern bei der Photodissoziation von $\mathrm{CH}_{2} \mathrm{ClI}$ in Lösung als mögliche Spezies vorgeschlagen, die sich jedoch erst aus Iodatomen, die den Lösungsmittelkäfig verlassen konnten und den Lösungsmittelmolekülen bildet [44]. Es ist bekannt, dass CCT-Komplexe besonders in Verbindung mit Iodatomen als Akzeptor aber auch mit anderen halogenhaltigen Spezies (z. B. $\mathrm{CH}_{3} \mathrm{I}$ als Donor) auftreten können, die im Bereich zwischen 300-400 nm absorbieren. Weil viele iodhaltige CCT-Komplexe aus der Literatur bekannt sind, scheint es schlüssig auch für diesen Fall eine solche Komplexbildung anzunehmen. Die Ausbildung dieses CCT-Komplexes aus dem hoch schwingungsangereten $\mathrm{CH}_{2} \mathrm{I}$ und dem Iod wird wahrscheinlich durch den Lösungsmittelkäfig stark unterstützt, da für die im Käfig verbleibenden Fragmente keine große räumliche Trennung möglich ist. Die Wechselwirkung beider Fragmente im Käfig könnte also 
ausreichen einen solch schwach gebundenen Komplex auszubilden, dessen Wechselwirkung jedoch groß genug für eine signifikante Absorption ist. Ladungstransferkomplexe (CT) zeigen dabei häufig sehr intensive Absorptionsbanden mit Extinktionskoeffizienten der Größenordnung $10^{4} \mathrm{lmol}^{-1} \mathrm{~cm}^{-1}$, wobei eine detaillierte theoretische Beschreibung der CT-Komplexe nur für die Gasphase besteht. Von CT-Komplexen in Lösung ist jedoch bekannt, dass das umgebende Lösungsmittel einen starken Einfluss auf die Absorption haben kann. So kann beispielsweise der Extinktionskoeffizient durch das Lösungsmittel erhöht werden, oder es kann zu einer Rotverschiebung der Bande durch eine destabilisierende Wirkung des Lösungsmittels kommen [171]. Außerdem können Reibungseffekte bei der relativen Bewegung der Komplexpartner auftreten.

In allen gemessenen, transienten Absorptionsspektren zu kurzen Zeiten wird eine zeitlich sehr schnell ansteigende und auch schnell wieder abfallende Bande bei etwa $355 \mathrm{~nm}$, außer in Xenon wo der schnelle Abfall nicht zu sehen ist, gefunden. Diese kann demnach von einem aus $\mathrm{CH}_{2} \mathrm{I}$ und I gebildeten CCT-Komplex stammen. Dieser würde sich schnell nach der Dissoziation durch Einfluss des Lösungsmittelkäfigs bilden und in der transienten Absorption sichtbar sein. Er wäre daher quasi eine absorbierende Intermediatspezies zur Bildung des $\mathrm{CH}_{2} \mathrm{I}$-I-Isomers. In den transienten Spektren von Åkesson und Mitarbeitern ist zu kurzen Zeiten ebenfalls eine Bandenstruktur im Bereich von $350 \mathrm{~nm}$ zu erkennen, die aber von ihnen dem schnell gebildeten Isomer zugeordnet wird [36]. Sollte der CCT-Komplex eine Intermediatspezies zur Bildung des Isomers sein, könnte auch spektral die Entwicklung der einen in die andere Spezies durch ein Abfallen und gleichzeitiges Anwachsen der entsprechenden Banden verfolgt werden. Dies kann aus den rekonstruierten Spektren jedoch nur qualitativ vermutet werden, da einerseits die spektrale Auflösung sehr gering ist, andererseits die Spektren nicht weit genug in den kurzwelligen Spektralbereich reichen.

\subsubsection{Zusammenfassung}

Die im Vorangegangenen diskutierten Hinweise auf die Beiträge zum transienten Kurzzeitsignal innerhalb der ersten Pikosekunden nach Photoanregungen haben gezeigt, dass vermutlich folgende Spezies beitragen: elektronisch angeregtes $\mathrm{CH}_{2} \mathrm{I}_{2}$, schwingungsheißes $\mathrm{CH}_{2} \mathrm{I}$ und ein möglicher $\mathrm{CH}_{2} \mathrm{I} \cdots$ I-CCT-Komplex. Zusätzlich können, wie erwähnt, noch kleinere Beiträge an kohärenter Lösungsmittelantwort innerhalb der ersten Pikosekunde eine Rolle spielen. 


\subsection{Einfluss des Schwingungsenergietransfers}

In diesem Abschnitt soll der Einfluss des Schwingungsenergietransfers des schwingungsheiß gebildeten $\mathrm{CH}_{2} \mathrm{I}-\mathrm{I}$-Isomers auf die Gestalt der transienten Spektren diskutiert werden.

Anhand der normierten, transienten Spektren lässt sich eine spektrale Dynamik erkennen, die auf einen Abkühlungsprozess hindeutet. Es lässt sich an der roten Flanke der Absorptionsbande eine zeitlich abfallende und im Maximum eine zeitlich ansteigende Komponente erkennen, so dass sich das Spektrum mit der Zeit spektral zu verengen scheint (siehe Abschnitte 4.2.2, 4.2.3, 4.2.4, 4.2.5). Dazwischen findet sich eine isosbestische Region, in der sich die Extinktion nicht mit der Zeit ändert. Dies deutet eindeutig auf eine Abkühlung der Spezies hin [142]. Die Abkühlung der heißen Spezies geschieht dabei über Schwingungsenergietransfer in das umgebende Lösungsmittel.

Die thermische Verteilung der Besetzung der Schwingungsniveaus im elektronischen Grundzustand eines Moleküls ändert sich mit der Temperatur. Dies entspricht einer mikrokanonischen Verteilung der Schwingungsenergie und damit einer der Verteilung äquivalenten Schwingungstemperatur, die für polyatomare Moleküle der makroskopischen Temperatur entspricht $[172,173]$. Auf Grund der Änderung der Besetzungszahlen der Schwingungsniveaus im Grundzustand durch Schwingungsenergietransfer in das umgebende Lösungsmittel ändert sich auch der Extinktionskoeffizient bei einer bestimmten Wellenlänge. Durch den Zusammenhang zwischen der Schwingungstemperatur und der mittleren inneren Energie des Moleküls (siehe Gleichung 5.2) kann die Änderung der mittleren inneren Energie des Moleküls über die Änderung des Extinktionskoeffizienten beschrieben werden [142]. Für zweiatomige Moleküle kann der Zusammenhang theoretisch nach dem Franck-Condon-Prinzip berechnet werden [142], was für $\mathrm{CH}_{2} \mathrm{I}_{2}$ nicht möglich ist.

Die Abhängigkeit kann auch über Kalibrierung, z.B. aus Stoßwellendaten, wie für das $\mathrm{CH}_{2} \mathrm{I}_{2}$ bereits beschrieben (siehe Abschnitt 5.1.2) [88], durch Messung thermisch heißer Spektren bei verschiedenen definierten Temperaturen erfolgen [143, 144, 174] die dann wiederum einer bestimmten mittleren inneren Energie entsprechen. Auf der langwelligen Flanke eines abkühlenden Spektrums wird ein nahezu linearen Verlauf des Extinktionskoeffizienten mit der inneren Energie beobachtet, was eine direkte Bestimmung des Schwingungsenergietransfers (VET) ermöglicht [88], wobei die Steigung des linearen Verlaufs zwischen mittlerer innerer Energie und dem Extinktionskoeffizienten dabei meist mit zunehmender Wellenlänge flacher wird [28]. Eine Kalibrierung der Extinktion gegen die innere Energie konnte bisher für das $\mathrm{CH}_{2} \mathrm{I}$-I-Isomer noch nicht durchgeführt werden, da die Lebensdauer des Isomers von einigen Nanosekunden nicht ausreicht, um Stoßwellenuntersuchungen durchzuführen oder auf anderem Wege thermische Spektren zu messen. 


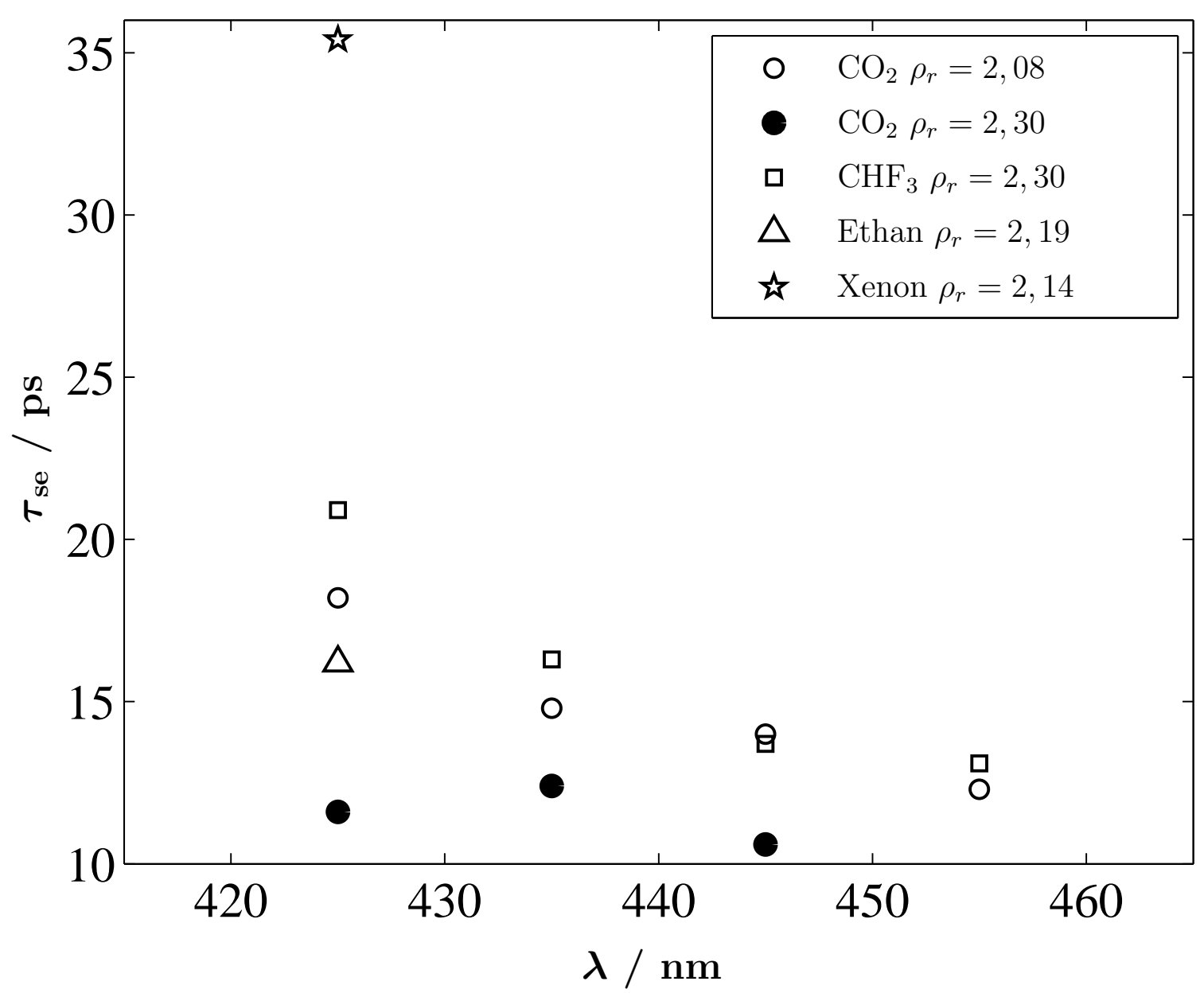

Abbildung 5.6: Aus den normierten Spektren ermittelte Abklingzeiten der spektralen Entwicklung $\tau_{\text {se }}$ aus den normierten Extinktionen bei verschiedenen Wellenlängen in den verwendeten Lösungsmitteln.

Für die langwellige Absorptionsflanke kann für das $\mathrm{CH}_{2} \mathrm{I}-\mathrm{I}$ aus den populationsnormierten Spektren bei einer Wellenlänge zu verschiedenen Zeitpunkten mit geringem Zeitabstand die Daten der normierten Absorbanz entnommen werden, um so den zeitlichen Verlauf der Absorbanz zu untersuchen. Die Werte werden den normierten Spektren entnommen, da diese die Änderung der Absorbanz durch zeitlichen Zuwachs an Isomer durch die Normierung nicht mehr beinhalten. Das zeitliche Verhalten der normierten Extinktion auf der roten Flanke der Absorptionsbande ist monoexponentiell, mit der Zeitkonstanten $\tau_{\text {se }}$, abfallend (siehe Abbildung 5.6) und wird mit zunehmender Wellenlänge schneller, was damit zu begründen ist, dass immer heißere Teile der Population beobachtet werden. Dieses beschreibt qualitativ das erwartete Verhalten des Schwingungsenergietransfers. So lassen sich einige qualitative Charakteristika bei Vergleich der Abklingzeiten $\tau_{\text {se }}$ in verschiedenen Lösungsmittel diskutieren: 
- Die Abfallzeiten werden zur roten Absorptionsflanke hin schneller, ändern sich dann aber nicht mehr so stark. Die auf der roten Flanke schneller werdende Abfallzeit ist auf das Abkühlen immer heißerer Moleküle der thermischen Verteilung zurückzuführen. Nach einer Umrechnung der normierten Absorptionsskala in eine Skala der inneren Energie sollten die daraus erhaltenen Energierelaxationszeiten vermutlich wellenlängenunabhängig sein.

- Die Abklingzeit wird mit zunehmender Dichte im $\mathrm{CO}_{2}$ schneller. Dies ist auf die mit der Dichte ansteigende Stoßzahl zurückzuführen [146].

- Die Abklingzeiten sind in $\mathrm{CO}_{2}$ schneller als in $\mathrm{CHF}_{3}$ bei vergleichbarer reduzierter Dichte. Ethan lässt sich etwas schwerer mit den anderen Lösungsmitteln vergleichen, da nur ein Vergleichspunkt zur Verfügung steht. Der Energietransfer scheint etwas langsamer als in $\mathrm{CO}_{2}$ zu sein aber auf jeden Fall schneller als in $\mathrm{CHF}_{3}$. Für Xenon kann jedoch festgestellt werden, dass der Energietransfer sehr viel langsamer ist als in den anderen Lösungsmitteln, was konsistent mit den Messungen von Kajimoto und Mitarbeitern ist, die für $\mathrm{CH}_{2} \mathrm{I}_{2}$ in Xenon im Bereich der reduzierten Dichte von $\rho_{\mathrm{r}}=0,6-1,6$ eine VET-Zeit von 200-600 ps erhalten haben [175], wobei anscheinend der VET des $\mathrm{CH}_{2} \mathrm{I}_{2}$ im Vergleich zum $\mathrm{CH}_{2} \mathrm{I}$-I-Isomer insgesamt langsamer ist. Dies lässt sich schon rein phänomenologisch über den beim Xenon nicht vorhandenen Schwingungs-SchwingungsEnergietransfer (V-V) begründen. In Xenon kann die Schwingungsenergie aus dem $\mathrm{CH}_{2} \mathrm{I}-\mathrm{I}$ nur über den Schwingungs-Translations-Energietransfer (V-T) abgeführt werden. Dieses kann mit Gasphasenexperimenten verglichen werden, in denen die mittlere pro Stoß übertragene Energie von Xenon über $\mathrm{CO}_{2}$ zu Ethan stark ansteigt [146]. Dies bedeutet, dass der Energietransfer ebenfalls schneller werden sollte. Ebenso kann dieser Trend für große, aromatische Systeme in überkritischen Fluiden bestätigt werden [146], jedoch können diese Ergebnisse nicht so ohne Weiteres auf das $\mathrm{CH}_{2} \mathrm{I}-\mathrm{I}$ übertragen werden, denn bei kleineren Molekülen wie $\mathrm{CH}_{2} \mathrm{I}_{2}$ ist der Energietransfer stark schwingungsmodenselektiv $[87,88,89,90]$. Dabei liefert nicht unbedingt die niederfrequenteste Schwingungsmode den größten Beitrag zum VET [90] sondern es kommt insbesondere auch auf die Kopplung der Solventmoden an die entsprechenden Solvatmoden an. So scheint die Kopplung der $\mathrm{CH}_{2} \mathrm{I}-\mathrm{I}$-Moden an die $\mathrm{CO}_{2}$-Moden sehr viel besser zu sein als an die $\mathrm{CHF}_{3}$-Moden.

- Der Energietransfer des $\mathrm{CH}_{2} \mathrm{I}-\mathrm{I}$ in $\mathrm{CO}_{2}$ scheint schneller zu sein als der des $\mathrm{CH}_{2} \mathrm{I}_{2}$ in $\mathrm{CO}_{2}$ [89]. Werden die Abklingzeiten an der roten Absorptionsflanke als grobe Abschätzung der VET Zeiten verwendet liegen diese bei einer reduzierten Dichte von $\rho_{\mathrm{r}}=2,08$ in $\mathrm{CO}_{2}$ bei etwa $12-15$ ps. Dagegen haben Kajimoto und Mitarbeiter die VET-Zeit für $\mathrm{CH}_{2} \mathrm{I}_{2}$ im Bereich von $\rho_{\mathrm{r}}=1,2-1,7$ auf einen Wert um $50 \mathrm{ps}$ bestimmt. Dieser Unterschied und der Unterschied in Xenon wäre durch das 
Vorhandensein von mehr niederfrequenten Moden im $\mathrm{CH}_{2} \mathrm{I}-\mathrm{I}$ erklärbar (siehe

Tabelle 2.1 auf Seite 14 und Tabelle 2.2 auf Seite 21).

\subsection{Lösungsmitteleinflüsse}

Wie schon anhand der experimentellen Ergebnisse in Kapitel 4 beschrieben beeinflusst die Lösungsmittelumgebung die Ausbeute und die Bildungsgeschwindigkeit des $\mathrm{CH}_{2} \mathrm{I}$-I-Isomers. In diesem Abschnitt soll daher genauer auf die Abhängigkeit der Dynamik von verschiedenen Lösungsmitteleigenschaften, wie insbesondere der Dichte und der elektrostatischen Wechselwirkungen eingegangen werden. Für den Vergleich der verschiedenen Lösungsmittel wurden die Messungen in den jeweiligen isosbestischen Regionen herangezogen.

\subsubsection{Ausbeute an Isomer}

Zunächst soll der Einfluss der Lösungsmitteldichte auf die Ausbeute an Isomer genauer beleuchtet werden. Als Maß für die Quantenausbeute $\phi_{\text {iso }}$ dient hier die Größe der Endamplitude $A_{\text {Anstieg }}$ des Signals. Zur Analyse der Dichteabhängigkeit der Isomerausbeute wird die Dissoziation des $\mathrm{CH}_{2} \mathrm{I}_{2}$ mit derjenigen des Iod im Lösungsmittelkäfig verglichen. Falls in Analogie zum Iodzerfall als Konkurrenzprozess zur Photoisomerisierung im Käfig nur das direkte Verlassen des Käfigs durch die Fragmente steht,

sollte die Ausbeute an Isomer und damit die Endamplitude $A_{\text {Anstieg }}$ der transienten Signale in den isosbestischen Regionen sich nahezu linear zu dem freien Volumen verhalten, das den Fragmenten nach der Dissoziation zur Verfügung steht. Das freie Volumen kann über die molekulare Packungsdichte und damit über die reduzierte Dichte $\rho_{r}=\rho / \rho_{c}$, also dem Verhältnis aus der Dichte zur kritischen Dichte beschrieben werden. Die kritischen Dichten zur Berechnung der reduzierten Dichten wurden ebenfalls dem NIST-WebBook [140] entnommen und belaufen sich auf $\rho_{c}\left(\mathrm{CO}_{2}\right)=10,63 \mathrm{~mol} / \mathrm{l}$, $\rho_{c}\left(\mathrm{CHF}_{3}\right)=7,50 \mathrm{~mol} / 1, \rho_{c}($ Ethan $)=6,87 \mathrm{~mol} / 1, \rho_{c}($ Xenon $)=8,37 \mathrm{~mol} / 1$.

Die in Abbildung 5.7 dargestellte Auftragung der Endamplitude und damit der relativen Ausbeute an $\mathrm{CH}_{2} \mathrm{I}-\mathrm{I}$ nach der Photodissoziation zeigt einen nahezu linearen Anstieg mit der reduzierten Dichte der Lösungsmittel. Außerdem ist das Verhalten mit der reduzierten Dichte, wie erwartet, nahezu unabhängig vom verwendeten Lösungsmittel. Lediglich die Messwerte für Ethan fallen dabei etwas heraus, was eventuell durch eine geringere Anregungsenergie im Vergleich zu den Messungen in $\mathrm{CO}_{2}$ und $\mathrm{CHF}_{3}$ erklärt werden könnte. Damit wäre eine direkte Vergleichbarkeit der Amplituden nicht unmittelbar gegeben. Um diese Fehlerquellen zu minimieren, besteht die Möglichkeit nicht die direkt gemessenen Amplituden zu vergleichen, sondern für den 


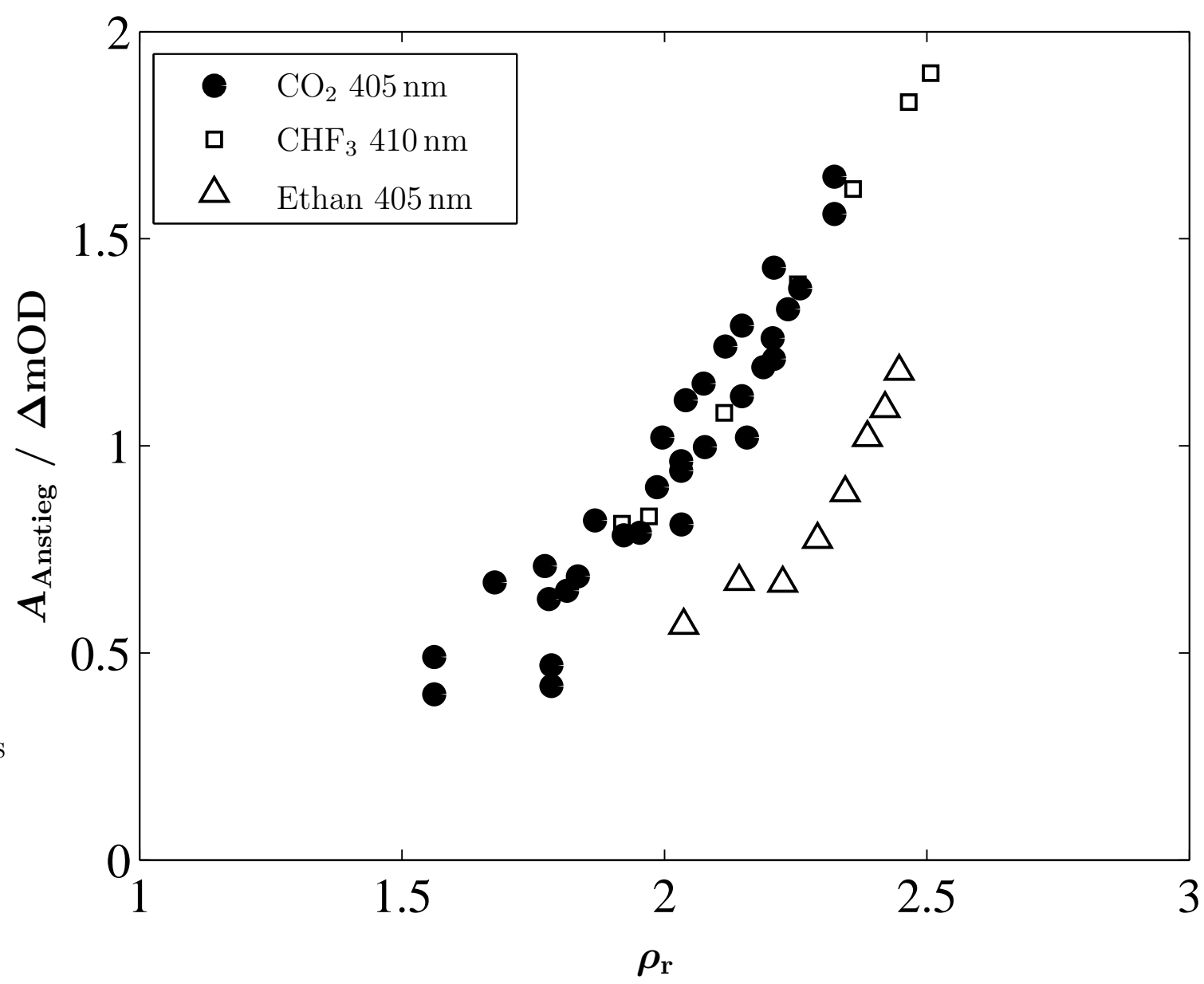

Abbildung 5.7: Abhängigkeit der Endamplitude $A_{\text {Anstieg }}$ des Signals von der reduzierten Dichte in verschiedenen überkritischen Lösungsmitteln.

Vergleich die normierten Amplituden $A_{\text {Anstieg,norm }}$ zu verwenden, d. h. die Endamplitude normiert auf die Höhe des ersten Absorptionsmaximums zu kurzen Zeiten. Dies sollte mögliche Unterschiede in der Anregungsenergie oder andere experimentelle Einflüsse auf die Gesamtsignalhöhe vernachlässigen. Eine Auftragung dieser normierten Amplitude findet sich in Abbildung 5.8.

Aus dieser Auftragung ist zu entnehmen, dass die Werte für die $\mathrm{CO}_{2}$ und Ethan Messungen nun sehr gut zusammenpassen, was darauf schließen lässt, dass es sich bei der Abweichung in der Auftragung der gemessenen Endamplitude gegen die reduzierte Dichte tatsächlich nur um eine experimentelle Eigenheit gehandelt hat. Bemerkenswerter ist jedoch, dass in dieser Auftragung die Werte in $\mathrm{CHF}_{3}$ mit den Werten in den anderen beiden Lösungsmitteln nicht mehr zusammenpassen. Dies könnte zwei möglich Gründe haben: 


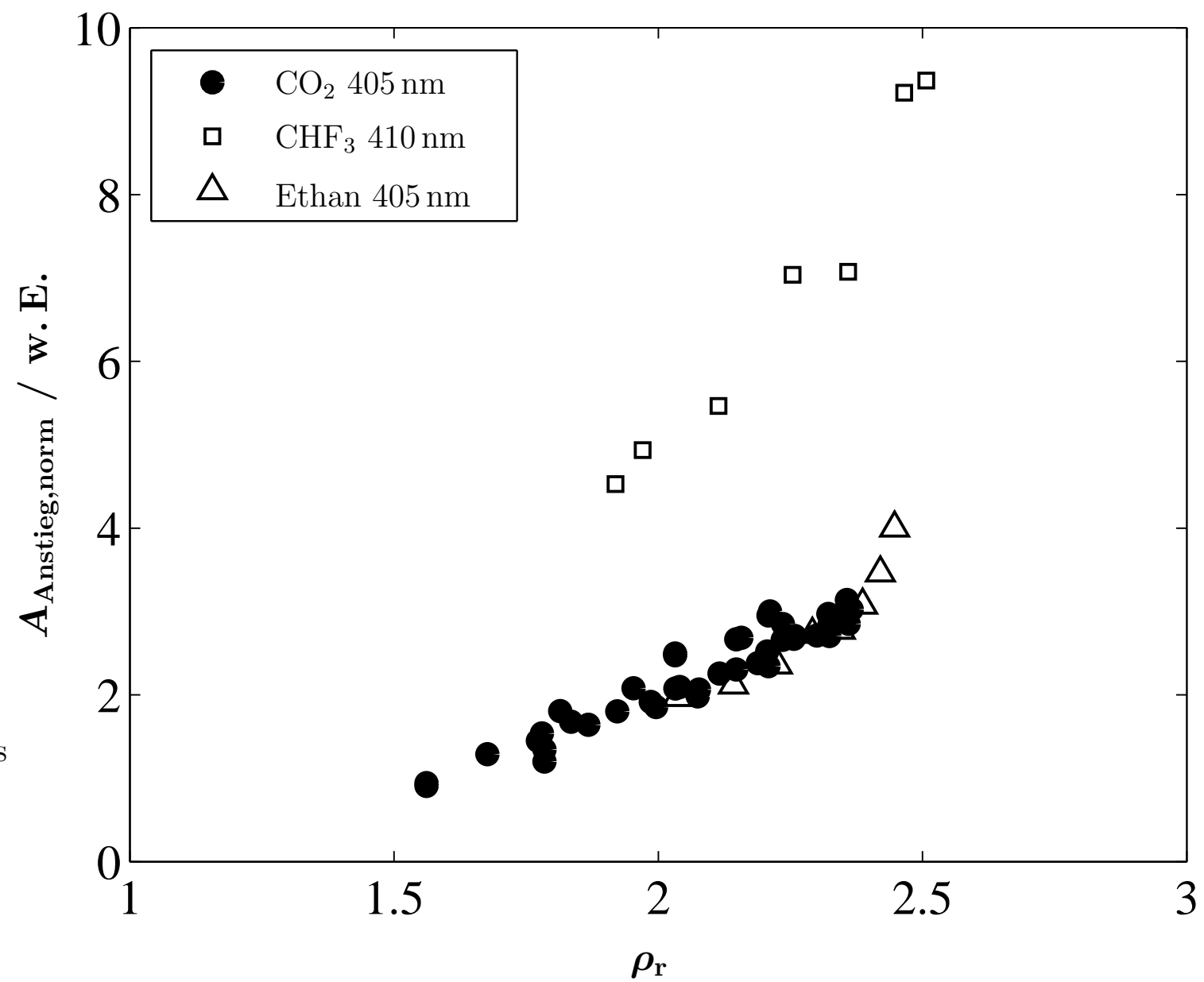

Abbildung 5.8: Abhängigkeit der normierten Endamplitude $A_{\text {Anstieg,norm }}$ des Signals von der reduzierten Dichte in verschiedenen überkritischen Lösungsmitteln.

1. Zum einen kann diese relative Erhöhung der Endamplitude zu den anderen beiden Lösungsmitteln auf ein kleineres erstes Absorptionsmaximum im Vergleich zur Endamplitude zurückzuführen sein. Dies würde bedeuten, dass im Wesentlichen die transiente Absorption des $\mathrm{CH}_{2} \mathrm{I}_{2}$ sich in $\mathrm{CHF}_{3}$ ändert, was wiederum eventuell auf die Polarität des Lösungsmittels zurückzuführen wäre.

2. Zum Anderen wäre eine Änderung des Extinktionskoeffizienten des $\mathrm{CH}_{2} \mathrm{I}-\mathrm{I}$ Isomers durch die Polarität des Lösungsmittels denkbar, die eine stärkere Absorption im polaren $\mathrm{CHF}_{3}$ im Vergleich zu den unpolaren Lösungsmitteln $\mathrm{CO}_{2}$ und Ethan bewirkt. Jedoch zeigen die transienten Signale ein merklich kleineres Signal im ersten Signalmaximum zu sehr kurzen Zeiten als im Vergleich zu den anderen Lösungsmitteln (siehe z. B. Abbildung 4.21 auf Seite 72). 
Es kann zunächst davon ausgegangen werden, dass der erwartete nahezu lineare Verlauf in Analogie zur Iod-Dissoziation bestätigt werden kann. Im Vergleich zwischen den unpolaren Lösungen $\mathrm{CO}_{2}$ und Ethan scheint die Ausbeute, analog zum Iodzerfall in unpolaren, überkritischen Fluiden [28], nur von der molekularen Packungsdichte abzuhängen. Die gleiche Abhängigkeit Isomerisierungsquantenausbeute von der Packungsdichte in Ethan und $\mathrm{CO}_{2}$ lässt zudem analog zum Iodzerfall den Schluss zu, dass es sich bei dem Verlassen des Lösungsmittelkäfigs durch die Fragmente um einen rein kinematischen Effekt handelt und ein diffusionskontrollierter Käfigausbruch nicht beobachtbar ist [28], weil $\mathrm{CO}_{2}$ und Ethan u. a. sehr verschiedene Diffusionskoeffizienten besitzen.

\subsubsection{Isomerisierungsgeschwindigkeit}

Im Folgenden soll genauer auf den Einfluss der verschiedenen Lösungsmitteleigenschaften auf die Isomerisierungsgeschwindigkeit eingegangen werden. Der Interpretation von Åkesson et al. folgend [36], ist die Anstiegszeit $\tau_{\text {Anstieg }}$ direkt mit der Isomerisierung korreliert. Wenn es sich bei der Geschwindigkeitskonstanten $k_{\text {Anstieg }}$ direkt um die Abkühlung, wie von Åkesson et al. vorgeschlagen, des schwingungsheiß gebildeten Isomers handeln würde, sollte in den isosbestischen Regionen keine sich über mehrere Pikosekunden erstreckende Anstiegszeit zu sehen sein, sondern es müsste nach der von ihnen postulierten Bildungsdauer von 1-2 ps für das $\mathrm{CH}_{2} \mathrm{I}$ - I-Isomer direkt die Endamplitude erreicht sein. Diese Bildungszeit im isosbestischen Bereich müsste demnach außerdem unabhängig von der Dichte sein. Dieses ist in den in Kapitel 4 vorgestellten Untersuchungen aber offensichtlich nicht der Fall, denn die Geschwindigkeitskonstante $k_{\text {Anstieg }}$ beträgt im Bereich der isosbestischen Region etwa 8-15 ps und steigt nahezu exponentiell mit der Dichte an.

Es kann zunächst vermutet werden, dass die Geschwindigkeitskonstanten von der sich mit der Dichte ändernden Lösungsmittelpolarisierbarkeit abhängt [148, 176], die evtl. einen Einfluss auf eine mögliche Reaktionsbarriere haben könnte [177, 178]. Das Maß für die Polarisierbarkeit ist dabei die molare Polarisation $P_{m}$ eines Stoffes, für die gemäß der Debye-Gleichung [179] bei Dichten oberhalb typischer Gasphasendichten gilt:

$$
P_{m}=\frac{\epsilon_{r}-1}{\epsilon_{r}+2} \cdot \frac{M}{\rho}=\frac{N_{\mathrm{A}}}{3 \epsilon_{0}}\left(\alpha+\frac{\mu^{2}}{3 k_{\mathrm{B}} T}\right),
$$

wobei $M$ die molare Masse, $\rho$ die Dichte und $N_{\mathrm{A}}$ die Avogadrokonstante bezeichnet. In der Gleichung beschreibt $\alpha$ den Anteil der Verschiebungspolarisation und $\frac{\mu^{2}}{3 k_{\mathrm{B}} T}$ den Anteil der Orientierungspolarisation, welcher für Lösungsmittel ohne permanentes Dipolmoment $(\mu=0)$ entfällt, wodurch die Gleichung in die so genannten ClausiusMosotti-Gleichung [179] übergeht. Für Lösungsmittel mit sehr kleiner relativer Per- 


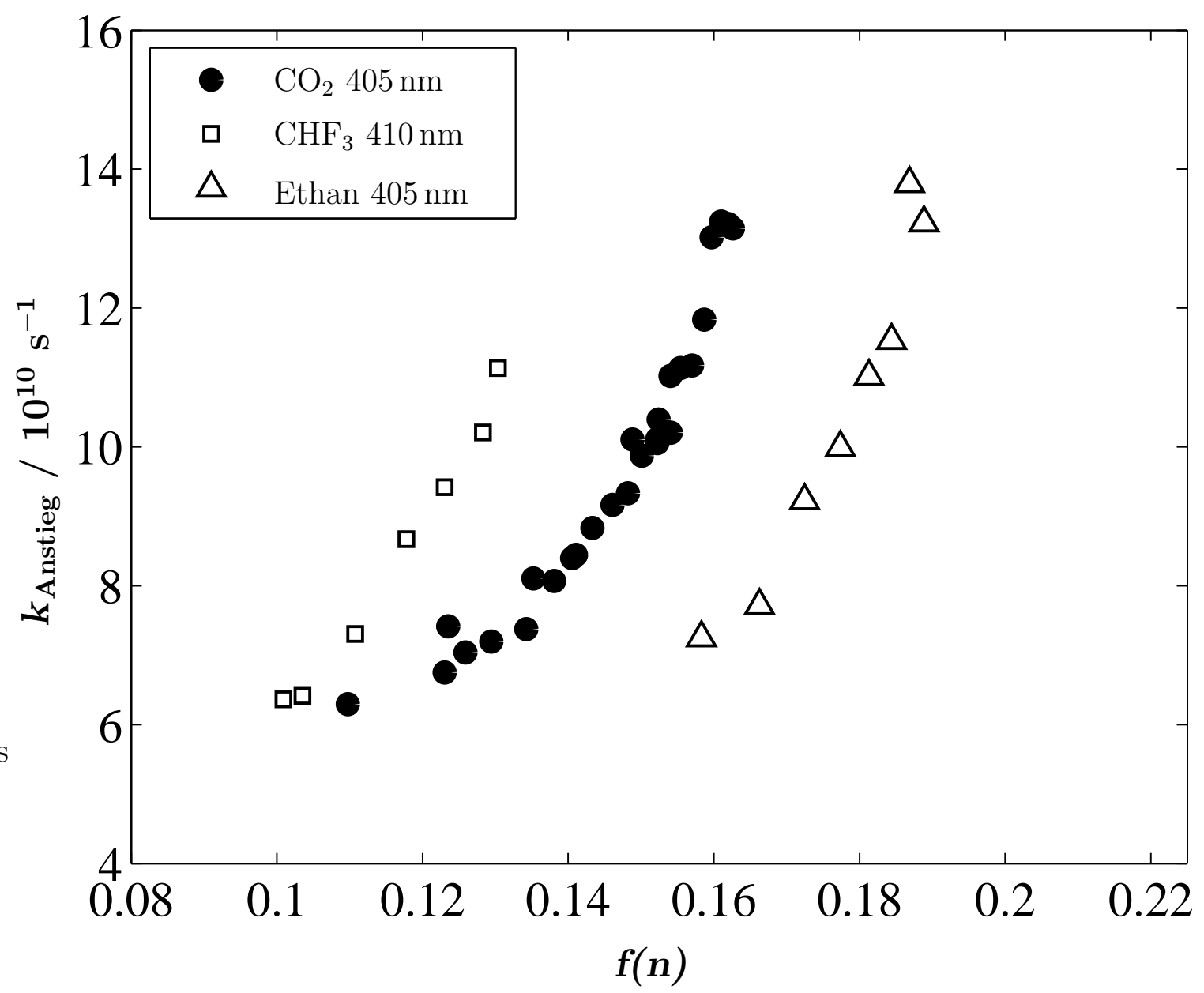

Abbildung 5.9: Abhängigkeit der Geschwindigkeitskonstanten $k_{\text {Anstieg }}$ des Anstiegs von dem Clausius-Mosotti-Faktor in verschiedenen überkritischen Lösungsmitteln.

meabilität $\epsilon_{r}$ kann über die Maxwellbeziehung $\epsilon_{r}(\nu)=n^{2}(\nu)$ die Debye-Gleichung als:

$$
P_{m}=\frac{n^{2}-1}{n^{2}+2} \cdot \frac{M}{\rho}=\frac{N_{\mathrm{A}}}{3 \epsilon_{0}}\left(\alpha+\frac{\mu^{2}}{3 k_{\mathrm{B}} T}\right)
$$

geschrieben werden. Für den Vergleich verschiedener Lösungsmittel kann als unabhängige Größe demnach der so genannte Clausius-Mosotti-Faktor:

$$
\begin{gathered}
f\left(\epsilon_{r}\right)=\frac{\epsilon_{r}-1}{\epsilon_{r}+2} \\
\text { bzW. } \\
f(n)=\frac{n^{2}-1}{n^{2}+2}
\end{gathered}
$$


verwendet werden. Die Berechnung des Clausius-Mosotti-Faktors in Abhängigkeit der Dichte wurde mit einer polynomischen Näherung nach Maroncelli et al. durchgeführt [180] (siehe Anhang A.3).

Die in Abbildung 5.9 zu findende Abhängigkeit der Geschwindigkeitskonstanten von dem jeweiligen Clausius-Mosotti-Faktor und somit von der Polarisierbarkeit des Lösungsmittels zeigt keine Lösungsmittelunabhängigkeit, so dass die vermutete Abhängigkeit der Geschwindigkeitskonstanten von der Polarisierbarkeit als alleinige Erklärung für die Dichteabhängigkeit der Geschwindigkeitskonstanten in den jeweiligen isosbestischen Regionen ausscheidet.

Dies lässt die Vermutung zu, dass es sich bei dem Einfluss der Dichte, genau wie bei den Isomerausbeuten, wiederum lediglich um einen Einfluss der Packungsdichte der Lösungsmittelmoleküle auf die Geschwindigkeitskonstante handelt.

Damit sollte die Geschwindigkeitskonstante $k_{\text {Anstieg }}$ bei einer Auftragung gegen die reduzierte Dichte $\rho_{\mathrm{r}}$ unabhängig vom gewählten Lösungsmittel sein. Dies ist nicht der Fall, wie Abbildung 5.10 zeigt. Die Geschwindigkeitskonstanten in Abhängigkeit von der reduzierten Dichte der Lösungsmittel liegen zwar dicht zusammen, sind aber nicht identisch. Dies könnte folgende Ursachen haben.

1. In der Reihenfolge ist der gleiche Trend wie in dem zeitlichen Verhalten für den VET zu erkennen. Diese Analogie und die auch in der isosbestischen Region mit der Dichte ansteigende Geschwindigkeitskonstante legen die Vermutung nahe, dass die Bildung des Isomers direkt an den Schwingungsenergietransfer gekoppelt ist. Danach wäre die Isomerisierung erst möglich, wenn genügend Energie, speziell aus dem $\mathrm{CH}_{2}$ I-Fragment, ins Lösungsmittel abgeflossen ist. Der VET als alleinige Ursache für den erhaltenen Anstieg der Geschwindigkeitskonstante mit der Dichte erscheint allerdings nicht ganz plausibel, denn bei einem rein durch Energietransfer bestimmten Prozess wäre eine lineare Abhängigkeit mit der Dichte zu erwarten [181].

2. Für den exponentiellen Anstieg der Geschwindigkeitskonstanten kann auch ein nicht durch die Polarisierbarkeit getriebener Einfluss der Packungsdichte des Lösungsmittels auf eine möglich Reaktionsbarriere angenommen werden. Eine genaue Analyse dieses Effektes ist aus den bisherigen Daten leider nicht möglich.

3. Als weitere Möglichkeit für das Verhalten der Geschwindigkeitskonstanten mit der Dichte kann ein Einfluss der lokalen Dichte angenommen werden. Dieser Möglichkeit soll in Abschnitt 5.3.3 nachgegangen werden. 


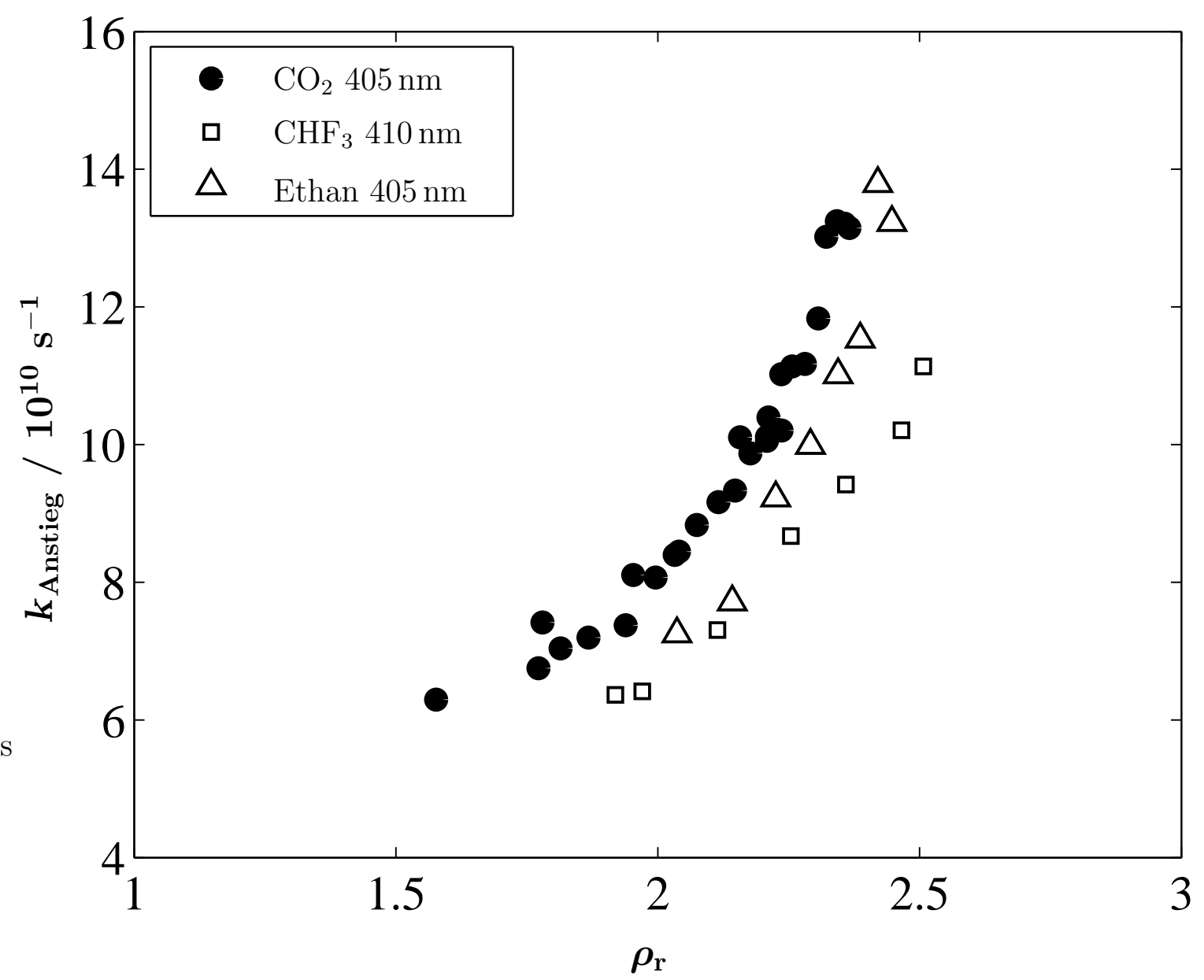

Abbildung 5.10: Abhängigkeit der Geschwindigkeitskonstanten $k_{\text {Anstieg }}$ des Anstiegs von der reduzierten Dichte in verschiedenen überkritischen Lösungsmitteln.

\subsubsection{Einfluss der lokalen Dichte}

In überkritischen Lösungsmitteln kann es zu Inhomogenitäten der Dichte um ein Solvatmolekül kommen [182]. Die lokale Dichte um ein Solvatmolekül kann dabei größer sein als die mittlere Dichte des reinen Lösungsmittels. Dieses Phänomen wird über die Entstehung von Solvat/Solventclustern auf Grund attraktiver Wechselwirkungen erklärt $[182,183]$. Dieser Effekt ist besonders bei Temperaturen leicht oberhalb der kritischen Temperatur besonders ausgeprägt, kann aber in einigen Fällen sogar bis zu $100 \mathrm{~K}$ oberhalb der kritischen Temperatur nachgewiesen werden [180, 181, 183, 184]. Die Abweichungen der lokalen Dichte von der reinen Lösungsmitteldichte sind für die meisten Solvate nur in einem Bereich von 0,6-1,5 der reduzierten Dichte von Relevanz und sind für $\mathrm{CO}_{2}, \mathrm{CHF}_{3}$ und Ethan meist sehr ähnlich $[180,184]$. 
Der Einfluss der lokalen Dichte auf Isomerausbeute und Isomerisierungsgeschwindigkeit könnte demnach in diesem Fall eine Rolle spielen, auch wenn es in dem Bereich 1,5-2,5 der reduzierten Dichte relativ unwahrscheinlich erscheint. Sollte die lokale Dichte einen Einfluss auf die Ausbeute und Geschwindigkeitskonstante haben, wäre in einem bestimmten Bereich der lokalen Dichte ein Abflachen bzw. ein Plateauverlauf der Dichteabhängigkeit der Parameter zu erwarten, da die lokale Dichte der Dichte des Solvat/Solventclusters entspricht und sich mit änderender Lösungsmitteldichte in einem gewissen Bereich nur sehr wenig ändert [180,184]. Sowohl der Verlauf der Amplitude

$A_{\text {Anstieg,norm }}$ (siehe Abbildung 5.8), die zu höheren reduzierten Dichten eventuell stärker ansteigt, als auch der scheinbare exponentielle Verlauf der Geschwindigkeitskonstanten $k_{\text {Anstieg }}$ (siehe Abbildung 5.10) könnten durch den Einfluss der lokalen Dichte entstanden sein und somit der Unterschied der Lösungsmittel eventuell doch nur durch den Unterschied der VET-Zeiten zustande gekommen sein [181], jedoch lässt sich dies anhand der bisher gewonnen experimentellen Daten nur schwer verifizieren. Daher sind weitere Untersuchungen nötig, die diese Möglichkeit ausschließen oder verifizieren.

\subsection{Mechanismen der Photoisomerisierung}

Der genaue kinetische Mechanismus der Käfigreaktionen nach der Photodissoziation des Diiodmethans im Lösungsmittelkäfig ist aus den bisher zur Verfügung stehenden Ergebnissen nicht quantitativ ableitbar. Für ein detailliertes kinetisches Modell sind noch zu viele Größen, die eine quantitative kinetische Modellierung zulassen, unbekannt. Es können zwar verschiedene Modelle aufgestellt werden, mit denen sich die gemessen Daten modellieren lassen, jedoch ist damit eine exakte Beschreibung des Mechanismus nicht zwangsläufig gegeben. Insbesondere die nicht verfügbaren Extinktionskoeffizienten der beteiligten Spezies sind kritisch, denn sowohl für das $\mathrm{CH}_{2} \mathrm{I}-\mathrm{I}$ Isomer als auch für den postulierten CCT-Komplex und für das $\mathrm{CH}_{2} \mathrm{I}$-Radikal sind keine Extinktionskoeffizienten bzw. keine temperaturabhängigen Spektren vorhanden, so dass beispielsweise keine exakten Quantenausbeute für die Photoisomerisierung angegeben werden können. Daher wird im Folgenden nur ein rein qualitatives kinetisches Modell aufgestellt, dass die Anschaulichkeit über die möglichen Prozesse erhöht (die 
eckigen Klammern deuten an, dass sich die Spezies im ursprünglichen Lösungsmittelkäfig befindet):

$$
\begin{array}{r}
\mathrm{CH}_{2} \mathrm{I}^{\ddagger}+\mathrm{I} \stackrel{k_{0}}{\longleftarrow}\left[\mathrm{CH}_{2} \mathrm{I}_{2}{ }^{*}\right] \stackrel{k_{1}}{\longrightarrow} \underset{\left.\mathrm{CH}_{2} \mathrm{I}^{\ddagger} \cdots \mathrm{I}\right]}{\left\lfloor k_{2}\right.} \stackrel{k_{3}}{\longrightarrow}\left[\mathrm{CH}_{2} \mathrm{I}_{2}^{\ddagger}\right] \\
\mathrm{CH}_{2} \mathrm{I}^{\ddagger}+\mathrm{I} \stackrel{k_{4}}{\longleftarrow} \quad\left[\mathrm{CH}_{2} \mathrm{I}^{\delta+} \ldots \mathrm{I}^{\delta-}\right] \\
\left\lfloor k_{5}\right. \\
{\left[\mathrm{CH}_{2} \mathrm{I}-\mathrm{I}^{\ddagger}\right]} \\
\left\lfloor k_{6}\right. \\
{\left[\mathrm{CH}_{2} \mathrm{I}-\mathrm{I}\right]}
\end{array}
$$

- Das nach Aufnahme eines Laserphotons angeregte $\mathrm{CH}_{2} \mathrm{I}_{2}{ }^{*}$ dissoziiert auf einer 100 fs Zeitskala in die Fragmente, schwingungsheißes $\mathrm{CH}_{2} \mathrm{I}^{\ddagger}$-Radikal und Iodatom. Dabei sind zwei Kanäle denkbar. Ein Kanal $\left(k_{0}\right)$, bei dem beide Fragmente sofort den Lösungsmittel impulsiv verlassen und einen Kanal $\left(k_{1}\right)$, bei dem das Fragmentpaar $\mathrm{CH}_{2} \mathrm{I}^{\ddagger} \cdots$ I zunächst im Lösungsmittelkäfig gebildet wird. Der erste Kanal wird für die kinetische Betrachtung eine sehr geringe Rolle spielen, da die kinetische Energie in den Fragmenten nur sehr gering ist, da der Hauptteil der Überschussenergie sich als innere Energie im Radikal wiederfindet. Dagegen spricht ferner die Tatsache dass ein Reibungseinfluss auf die Dissoziation zu erkennen ist (siehe Abschnitt 5.1.2).

- Dem $\mathrm{CH}_{2} \mathrm{I}^{\ddagger} \ldots$ I-Fragmentpaar eröffnen sich wiederum zwei weitere mögliche Reaktionskanäle. Zum einen der Kanal $\left(k_{3}\right)$ zur Rekombination zu schwingungsheißem $\mathrm{CH}_{2} \mathrm{I}_{2}^{\ddagger}$-Isomer und zum anderen zur Bildung des $\mathrm{CH}_{2} \mathrm{I}^{\delta+} \ldots \mathrm{I}^{\delta-}$ CCT-Komplexes $\left(k_{2}\right)$. Die Rekombinationsreaktion $\mathrm{zu} \mathrm{CH}_{2} \mathrm{I}_{2}^{\ddagger}$ ist, wie in Abschnitt 5.1.2 diskutiert, aus energetischen und sterischen Gründen nur sehr langsam möglich und spielt für die kinetische Betrachtung keine Rolle, da der Reaktionskanal zum CCT-Komplex klar dominiert.

- Aus dem schwach gebundenen CCT-Komplex kann wiederum das schwingungsheiße $\mathrm{CH}_{2} \mathrm{I}-\mathrm{I}^{\ddagger}$ gebildet werden $\left(k_{5}\right)$. Dieser Prozess zeigt eine Dichteabhängigkeit über deren Ursprung noch keine Klarheit herrscht (siehe Abschnitt 5.3). Es wird ferner davon ausgegangen, dass die Rückreaktion von einmal gebildetem Isomer sehr unwahrscheinlich ist, da es relativ schnell stabilisiert wird.

- Das schwingungsheiß gebildete Isomer kühlt anschließend über VET in das umgebende Lösungsmittel ab $\left(k_{6}\right)$ (siehe Abschnitt 5.2).

- Als Konkurrenz zu der Isomerisierung kann das Verlassen des Lösungsmittelkäfigs durch das Radikalpaars als Kanal auftreten $\left(k_{4}\right)$, wobei eine Rückreaktion über diesen Kanal nicht ins Gewicht fällt, da einmal separierte Fragmente erst 
viel später (100 ps-Zeitskala) wieder aufeinander treffen können. Die Geschwindigkeit des Verlassens des Käfigs ist ebenfalls dichteabhängig und wirkt sich auf die Quantenausbeute an gebildeten Isomer aus, die sich linear mit der reduzierten Dichte verhält (siehe Abschnitt 5.3). Für die Geschwindigkeitskonstanten der Dissoziation und der Bildung des CCT-Komplexes kann davon ausgegangen werden, dass sie sehr viel schneller als Isomerbildung und Verlassen des Käfigs sind, so dass der kinetische Mechanismus hauptsächlich von der Konkurrenz zwischen Verlassen des Käfigs und Bildung des Isomers bestimmt wird. Dieser vorgeschlagene Mechanismus könnte auch für die Abweichungen der $\mathrm{CHF}_{3}$-Messungen verantwortlich sein (siehe Abschnitt 5.3). Es lässt sich vermuten, dass der postulierte CCT-Komplex in dem polaren Lösungsmittel $\mathrm{CHF}_{3}$ besser stabilisiert wird als in den anderen Lösungsmitteln. Dadurch würde $k_{4}$ und damit auch die Observable $k_{\text {Anstieg }}$ kleiner werden und die Ausbeute an Isomer ansteigen, weil die Isomerisierung gegenüber der Separation der Fragmente begünstigt wäre.

Für eine weitergehende genauere Analyse des Mechanismus sind, wie bereits erwähnt, die Extinktionskoeffizienten der beteiligten Spezies nötig. Um die Komplexität des Mechanismus zu verringern, sind Messungen bei geringeren Lösungsmitteldichten notwendig, bei denen der Kanal zur Isomerbildung geschlossen sein könnte. Eine detaillierte Diskussion weiterer möglicher Experimente zur Klärung der offenen Fragen findet sich im folgenden Kapitel 6.

\subsection{Abschließende Bemerkungen}

Die in Kapitel 1 gestellten Fragestellungen konnten in dieser Arbeit zwar nicht erschöpfend beantwortet werden, jedoch konnten eine ganze Reihe von Indizien und Hinweisen zur Aufklärung des Mechanismus der Konkurrenz zwischen Photodissoziation und Photoisomerisation des Diiodmethans in Lösung gegeben werden.

Aus den bisherigen Ergebnissen ist in vielen Aspekten eine deutliche Analogie zum Zerfall des Iodmoleküls zu erkennen, so dass in großen Teilen der Interpretation auf die beim Iod sehr gut bekannten Vorgänge zurückgegriffen werden konnte. Insbesondere die Dichteabhängigkeit der Ausbeute an Isomer zeigt eine deutliche Analogie zur Dichteabhängigkeit der Dissoziationsquantenausbeute bei der Iod-Photodissoziation.

Weiterhin konnten wichtige phänomenologische Erkenntnisse über die Art des Schwingungsenergietransfers des $\mathrm{CH}_{2} \mathrm{I}$-I-Isomers und über die, zu kurzen Verzögerungszeiten zur transienten Absorption beitragenden, Spezies gewonnen werden.

Eine wichtige Fragestellung dieser Arbeit konnte bislang noch nicht engültig geklärt werden: der Beginn der Öffnung des Reaktionskanals zur Isomerisierung beim Übergang von Gasphasen- zu Flüssigkeitsdichten im überkritischen Medium. Jedoch werden 
im folgenden Kapitel 6 weitere experimentelle und theoretische Untersuchungen vorgeschlagen, um diese und weitere offene Fragen zu klären und das bisherige Bild zu komplettieren. 


\section{Kapitel 6}

\section{Ausblick}

Dieses Kapitel beschäftigt sich mit experimentellen und theoretischen Möglichkeiten die Untersuchungen zu den Mechanismen der Photodissoziation und Photoisomerisierung des $\mathrm{CH}_{2} \mathrm{I}_{2}$ im Lösungsmittelkäfig auszuweiten, um weitere Rückschlüsse auf dynamische und kinetische Aspekte des gesamten Reaktionsmechanismus ziehen zu können.

\subsection{Messungen bei niedrigeren Dichten}

Eines der Schlüsselexperimente, wäre, wie bereits angesprochen (vergleiche Abschnitt 5.4 auf Seite 111), die Untersuchung der transienten Absorption bei geringeren Dichten. Da es in der Gasphase nach Photodissoziation des $\mathrm{CH}_{2} \mathrm{I}_{2}$ zu keiner Isomerbildung kommt, wäre zu erwarten, dass es bei Verringerung der Dichte in den Bereich von typischen Gasphasendichten einen Bereich gibt, bei dem der Reaktionskanal zum Isomer nicht mehr offen ist. Bei etwa 100 bar und $308 \mathrm{~K}$ wurde dieser Bereich in $\mathrm{CO}_{2}$ bislang noch nicht erreicht. Jedoch stößt man bei diesen Bedingungen bereits an experimentelle Grenzen, weil sich das $\mathrm{CH}_{2} \mathrm{I}_{2}$ bei geringeren Drücken bei dieser Temperatur nicht mehr vollständig im $\mathrm{CO}_{2}$ löst. Daher ist es notwendig, die Temperatur der Zelle zu erhöhen, um bei gleichem Druck eine geringere Dichte und ein besseres Löslichkeitsverhalten $\mathrm{zu}$ erhalten. In der bisher verwendeten Version ist die Hochdruckzelle nur bis ca. $40^{\circ} \mathrm{C}$ verwendbar. Diese Temperatur reicht jedoch noch nicht aus, um die Löslichkeit entscheidend zu verbessern. Daher wurde für weitere Experimente eine leicht modifizierte Zelle konzipiert, bei der die Halterung der Fenster nicht mehr durch einen Klebstoff, sondern durch ein Verschraubungssystem realisiert ist. Erste Voruntersuchungen mit dieser Zelle waren bislang recht vielversprechend, wobei das Problem des Auskondensierens des $\mathrm{CH}_{2} \mathrm{I}_{2}$ und der anderen Photoprodukte an den Fensteroberflächen auf Grund des herrschenden Temperaturgradienten an den Fensteroberflächen noch zu lösen ist. Alternativ könnte in einer Zelle mit längerer Schichtlänge und einer 
kollinearen Pump/Probe-Anordnung gearbeitet werden, die jedoch den Nachteil hätte, dass die Zeitauflösung des Experimentes sich drastisch verschlechtern würde.

Sollten Messungen bei Dichten möglich sein, bei denen der Isomerisierungskanal nicht mehr offen ist, könnte Klarheit darüber gewonnen werden, ob z. B. das Radikal einen Beitrag zum Anfangssignal liefert. Falls das schwingungsheiße $\mathrm{CH}_{2}$ I-Radikal im Bereich zwischen 300-400 nm einen Beitrag zum transienten Kurzzeitsignal hat, sollte dies detektierbar sein. Es wäre zu erwarten, dass es nach der schnellen Bildung durch den Dissoziationsprozess auf einer ps-Zeitskala abkühlt. Dieser Abkühlungsprozess sollte sowohl in den Absorptionszeitprofilen als auch in transienten Spektren sichtbar sein.

\subsection{Spektrale Entwicklungen}

Desweiteren werden zusätzliche spektral und zeitaufgelöste Messungen vorgeschlagen. Die spektralen Entwicklungen konnten in dieser Arbeit nur aus rekonstruierten Spektren gewonnen werden. Die spektrale Auflösung ist nur sehr gering, da auf Grund der langen Messzeiten für ein Absorptions-Zeitprofil nur ein Abstand von etwa $10 \mathrm{~nm}$ gewählt werden konnte. Weiterhin konnten im Bereich um den ersten Oberton der Laserfundamentalen bei $388 \mathrm{~nm}$ keine verlässlichen Messungen durchgeführt werden. Der Abgleich der relativen Intensitäten ist nur ungenau durchführbar, da oft eine Nachoptimierung des räumlichen Überlapps zwischen Pump- und Probestrahl nötig war. Die rekonstruierten Spektren sind nur bis zu einer Wellenlänge $\geq 350 \mathrm{~nm}$ verfügbar, da zu kürzeren Wellenlängen ein zu großer Anteil des Probelichtes bereits vom $\mathrm{CH}_{2} \mathrm{I}_{2}$ absorbiert wird.

Das vorhandene Spektrometer könnte durch eine Anordnung zum Proben mit Weißlicht (supercontinuum probe) ergänzt werden. Dabei wird durch ein in einem nichtlinearen Prozess erzeugtes Weißlichtkontinuum verwendet, um bei fester Verzögerungszeit das gesamte Spektrum in einem Laserschuss aufzunehmen. Derzeit verwendete UVPump/Weißlicht-Probe-Apparaturen können einen spektralen Bereich von 300-900 nm proben, wobei meist ein Photodiodenarray mit 512 Bildpunkten zur Detektion eingesetzt wird [185]. Der Einsatz einer solchen Technik für die $\mathrm{CH}_{2} \mathrm{I}_{2}$-Untersuchungen wür-

de die spektrale Auflösung und die spektrale Bandbreite deutlich erhöhen. Jedoch ist das Grundrauschen dieser Apparaturen deutlich höher als bei dem in dieser Arbeit verwendeten Zwei-Farben-Pump/Probe-Systems und liegt meist um $\Delta O D= \pm 0,001$ [185]. Ein Einsatz dieser Technik würde auf Grund der kleinen Signale demnach zu einer Verschlechterung des Signal-zu-Rauschverhältnisses (signal-to-noise-ratio, SNR) führen und so die Untersuchung erheblich erschweren. Bei einer jedoch noch möglichen Erhöhung der Pulsenergie des Pumppulses wären Messungen mit akzeptablem SNR denkbar. 
Falls solche Breitband-Messungen durchführbar sind, könnten daraus weitere Erkenntnisse gewonnen und noch besser mit transienten Spektren von Åkesson et al. in flüssiger Phase verglichen werden, die ebenfalls eine ähnliche Breitband-Technik eingesetzt haben [36]. Bei einer besseren spektralen Auflösung könnte die spektrale Entwicklung der transienten Absorptionsbanden detailliert verfolgt werden. Eine Normierung auf die Fläche unter der Bande bei einer bestimmten Verzögerungszeit wäre dann deutlich aussagekräftiger. Daraus ließe sich der Einfluss der Schwingungsrelaxation auf die spektrale Entwicklung noch besser untersuchen. Ferner könnte evtl. der Beitrag der Radikalabsorption im Bereich von 300-350 nm aufgelöst werden. Außerdem könnte im gleichen Spektralbereich geklärt werden, ob tatsächlich der postulierte CCT-Komplex einen Beitrag zur Absorption liefert. Falls dieser eine Vorläuferspezies zur Isomerbildung darstellt, müsste im Spektrum eine zeitliche Entwicklung der Absorption der einen Spezies in die andere sichtbar sein.

\subsection{Messungen in der zweiten Absorptionsbande des $\mathrm{CH}_{2} \mathrm{I}-\mathrm{I}$}

Zur Aufklärung der Reaktionsmechanismen könnte die Untersuchung des transienten Absorptionsverhaltens in der zweiten Absorptionsbande des $\mathrm{CH}_{2} \mathrm{I}-\mathrm{I}$ bei $570 \mathrm{~nm}$ beitragen. Im Bereich dieser Bande sollte der Beitrag der $\mathrm{CH}_{2}$ I-Radikalabsorption vernachlässigbar sein.

Eine Untersuchung der spektralen Dynamik, evtl. mit dem im vorangegangenen Abschnitt vorgeschlagenen Weißlicht-Probe-System (siehe Abschnitt 6.2), würde nach Abklingen einer möglichen transienten $\mathrm{CH}_{2} \mathrm{I}_{2}$-Absorption nur noch Beiträge des $\mathrm{CH}_{2} \mathrm{I}-\mathrm{I}$ Isomers liefern. Anhand der daraus gewonnenen transienten Spektren könnte der Einfluss des Schwingungsenergietransfers in das Lösungsmittel auf die Konkurrenz zwischen Photodissoziation und Photoisomerisierung noch besser untersucht werden.

Desweiteren könnten Anisotropiemessungen im Bereich der zweiten Absorptionsbande Aufschluss darüber geben, ob, wie vermutet, das $\mathrm{CH}_{2}$ I-Radikal Ursprung der Orientierungsrelaxation ist. In diesem Spektralbereich wäre zu vermuten, dass selbst sehr schwingungsheißes Radikal keinen sichtbaren Einfluss auf die transiente Absorption mehr haben sollte. Falls sich also ein ähnliches Anisotropieverhalten ergeben würde, wie in der ersten Absorptionsbande, müsste die Interpretation neu überdacht werden.

Im Bereich der zweiten Absorptionsbande wären auch Experimente mit höherer Zeitauflösung möglich. Dazu könnte die Fundamentale des NOPA bei etwa $600 \mathrm{~nm}$ als Abfrage und die zweite Harmonische bei $300 \mathrm{~nm}$ als Anregung verwendet werden. Mit einer so verbesserten Zeitauflösung von etwa 20-50 fs wäre es möglich die schnelle 
abfallende Komponente nach dem ersten Absorptionsmaximum, welches im Bereich von $600 \mathrm{~nm}$ ebenfalls deutlich vorhanden sein müsste [35,36], genauer zu untersuchen. Daraus könnte der Einfluss der Reibung auf die Dissoziation des $\mathrm{CH}_{2} \mathrm{I}_{2}$ durch die Lösungsmittelviskosität (siehe Abschnitt 5.1.1 auf Seite 90) innerhalb kleinerer Fehlergrenzen untersucht werden.

\subsection{Zerfallsreaktion des Isomers}

Weiteren Aufschluss auf den Mechanismus der Photoisomerisierung des $\mathrm{CH}_{2} \mathrm{I}_{2}$ zum $\mathrm{CH}_{2} \mathrm{I}-\mathrm{I}$ könnten Messungen zum Zerfall des $\mathrm{CH}_{2} \mathrm{I}-\mathrm{I}$-Isomers geben. Das Isomer scheint eine Lebensdauer von einigen $100 \mathrm{~ns}$ zu besitzen [35], so dass einer Bestimmung der Lebensdauer dieser Spezies mit dem in dieser Arbeit vorgestellten Pump/Probesystem nicht möglich ist. Es könnten allerdings Pump/Probemessungen mit ns-Zeitauflösung z. B. mit einem XeCl-Excimerlaser als Pumpquelle und einer $\mathrm{Xe} / \mathrm{Hg}$-Hochdrucklampe als Probe verwendet werden, um die Lebensdauer zu bestimmen. Aus diesen Messungen könnten evtl. Rückschlüsse auf eine möglich Reaktionsbarriere gewonnen werden. Die zur Zeit noch unklaren Zerfallskanäle des $\mathrm{CH}_{2} \mathrm{I}$-I-Isomers könnten mit den vorgeschlagenen Untersuchungen aufgeschlüsselt werden.

Für die Durchführung dieser Untersuchungen stünde in der Abteilung eine derartige Apparatur mit einer heizbaren Hochdruckzelle und einer schnellen Messelektronik zur Verfügung, mit der eine Zeitauflösung von 10-20 ns möglich wäre [186].

\subsection{Messung thermischer Spektren}

Für die Verifizierung, dass das $\mathrm{CH}_{2}$ I-Radikal zur transienten Kurzzeitdynamik beiträgt und zur genauen Berechnung der Quantenausbeuten sowie die Bestimmung exakter VET-Zeiten, wäre eine genaue Kenntnis der Absorptionsspektren des $\mathrm{CH}_{2}$ I-Radikals sowie des $\mathrm{CH}_{2} \mathrm{I}$-I-Isomers bei verschiedenen Temperaturen ausgesprochen hilfreich. Daraus könnte eventuell analog zum $\mathrm{CH}_{2} \mathrm{I}_{2}$ eine Kalibrierung des Extinktionskoeffizienten bei einer Wellenlänge mit der inneren Energie vorgenommen werden und zu höheren inneren Energien extrapoliert werden, um den Extinktionskoeffizienten bei der inneren Energie des Radikals direkt nach der Dissoziation zu bestimmen.

Zur Durchführung dieser Untersuchungen könnte ebenfalls die bereits genannte oder eine weitere in der Abteilung vorhandene Apparatur mit ns-Zeitauflösung dienen. Dabei kann mit einem Excimerlaser das $\mathrm{CH}_{2} \mathrm{I}_{2}$ in der heizbaren Gaszelle photolysiert und 
mit einer Hochdruck-Gasentladungslampe und einem Spektrometer das Spektrum gemessen werden. Die Detektion erfolgt dabei über eine ICCD-Kamera mit einer Zeitauflösung von $\sim 1$ ns. Durch Subtraktion des jeweiligen $\mathrm{CH}_{2} \mathrm{I}_{2}$-Absorptionsspektrums kann so das $\mathrm{CH}_{2} \mathrm{I}$-Spektrum in der Gasphase bei verschiedenen Temperaturen erhalten werden. Außerdem könnten mit einem ähnlichen Verfahren eventuell temperatur- und zeitabhängige Spektren des $\mathrm{CH}_{2} \mathrm{I}$-I-Isomers aufgenommen werden, um z. B. die spektrale Entwicklung des Isomerzerfalls zu detektieren. Eine Kalibrierung des Extinktionskoeffizienten für Radikal und Isomer kann im Vergleich zu Stoßwellenmessungen jedoch nur über einen kleinen Temperaturbereich durchgeführt werden, da der untersuchbare Temperaturbereich nur bis etwa $900 \mathrm{~K}$ reicht [186,187]. Allerdings könnten Untersuchungen in der reinen Dampfphase des $\mathrm{CH}_{2} \mathrm{I}_{2}$ und einer Verzögerungszeit im Bereich von 1 ns die Möglichkeit eröffnen unter quasi stoßfreien Bedingungen direkt das Spektrum des schwingungsheißen Radikals aus der Photodissoziation zu erhalten.

\subsection{Einfluss der lokalen Dichte}

Die Aufklärung eines möglichen Einflusses der lokalen Dichte auf die Dichteabhängigkeit der Quantenausbeute und der Geschwindigkeitskonstanten der Isomerisierung könnte über die Erweiterung der Untersuchungen auf einen Bereich niedrigerer reduzierter Lösungsmitteldichten erreicht werden. Dazu könnte, wie bereits vorgeschlagen (siehe Abschnitt 6.1) eine überarbeite Version der Hochdruckmesszelle dienen, die es ermöglicht in geringere Lösungsmitteldichtebereiche vorzudringen. Diese Messungen könnten mit einer Simulationsrechnung der lokalen Dichte verglichen werden $[182,184]$.

Für eine theoretische Modellierung der lokalen Dichte wäre eine Mittelung der verschiedenen isolierten Solvat-Solvent-Wechselwirkungen notwendig. Dafür werden allerdings u. a. Lennard-Jones-Parameter des Solvates und des Solvent benötigt, um die intermolekularen Wechselwirkungen z.B. über radiale Verteilungsfunktionen zu berechnen [184]. Dazu müssten die Lennard-Jones-Parameter und die Coulomb-Wechselwirkung mit ab initio oder semiempirischen Methoden berechnet werden, da auf Grund der Lebensdauer des $\mathrm{CH}_{2} \mathrm{I}$-I-Isomers eine spektroskopische Bestimmung der Parameter nicht möglich erscheint.

\subsection{Untersuchung anderer Dihalomethane}

Aussichtsreichster Kandidat für weitere Untersuchungen an verwandten Dihalomethanverbindungen ist das Chloriodmethan $\mathrm{CH}_{2} \mathrm{ClI}$. Für das $\mathrm{CH}_{2} \mathrm{ClI}$ wurde analog zum $\mathrm{CH}_{2} \mathrm{I}_{2}$ von Maier und Mitarbeitern in Matrixmessungen ein Photoisomer $\left(\mathrm{CH}_{2} \mathrm{Cl}-\mathrm{I}\right)$ 
gefunden [33]. Auch in flüssiger Lösung konnte in fs-Pump/Probe-Messungen von Akesson und Mitarbeitern [43], sowie in Resonanz-Raman-Messungen von Phillips und Mitarbeitern [44] das $\mathrm{CH}_{2} \mathrm{Cl}-\mathrm{I}$-Isomer nachgewiesen werden.

Aus experimenteller Sicht wäre das $\mathrm{CH}_{2} \mathrm{ClI}$ gut untersuchbar. Es verfügt über eine breite, nahezu gaußförmige stationäre Absorptionsbande, die um $260 \mathrm{~nm}$ zentriert ist [44]. Eine Anregung in das Maximum dieser Bande wäre mit der dritten Harmonischen der Laserfundamentalen bei $258 \mathrm{~nm}$ problemlos möglich. Die transienten Absorptionsbanden des $\mathrm{CH}_{2} \mathrm{Cl}$-I-Isomers liegen im Bereich von $460 \mathrm{~nm}$ und $710 \mathrm{~nm}$ [43]. Diese Wellenlängen sind ebenfalls experimentell gut zugänglich und für spektral aufgelöste Messungen gäbe es keine Lücke im Bereich einer Harmonischen der Laserfundamentalen.

Bei der Anregung in das erste Absorptionsmaximum bei $260 \mathrm{~nm}$ wird nur der C-I nicht aber der C-Cl-Chromophor angeregt, so dass nur die C-I-Bindung brechen kann [36]. Die aus der Dissoziation entstehenden Fragmente, schwingungsheißes $\mathrm{CH}_{2} \mathrm{Cl}$-Radikal und I sollten im Vergleich zum $\mathrm{CH}_{2} \mathrm{I}_{2}$ einen größeren Teil der verfügbaren Energie in Translationsenergie zur Verfügung haben [46]. Daraus ergibt sich gegebenfalls eine andere Dynamik und so evtl. ein andere Viskositätsabhängigkeit der Photodissoziation als beim $\mathrm{CH}_{2} \mathrm{I}_{2}$ die untersuchbar wäre. Ferner sollte das $\mathrm{CH}_{2} \mathrm{Cl}$-Radikal erst bei sehr viel kürzeren Wellenlängen im UV absorbieren, so dass die Absorption dieser Spezies im Bereich der Isomerbanden keine Rolle spielen sollte. Ein ebenfalls möglicher $\mathrm{CH}_{2} \mathrm{Cl} \cdots$ I-CCT-Komplex, der innerhalb des Lösungsmittelkäfigs gebildet werden könnte, würde auch in einem anderen spektralen Bereich absorbieren als der für den $\mathrm{CH}_{2} \mathrm{I}_{2}$-Zerfall postulierte CCT-Komplex. Da das $\mathrm{CH}_{2} \mathrm{Cl}$-I-Isomer scheinbar eine sehr viel kürzere Lebensdauer hat und nach einer Verzögerungszeit von etwa 100 ps in transienten Messungen bereits wieder zu zerfallen beginnt [43] können für das $\mathrm{CH}_{2} \mathrm{Cl}-\mathrm{I}$ eventuell besser Informationen über mögliche Reaktionsbarrieren und über andere dynamische und kinetische Aspekte der Photoisomerisierungsreaktion gewonnen werden. 


\section{Anhang}

\section{A.1 Modellfunktionen des Anpassungsprogramms}

Die im Folgenden aufgelisteten Modellfunktionen standen für die Anpassung mit dem, in Abschnitt 3.4.3 beschriebenen Matlab basierten, Auswertprogramm zur Verfügung:

1. Monoexponentieller Abfall

2. Monoexponentieller Anstieg

3. Monoexponentieller Abfall + Monexponentieller Anstieg

4. Biexponentieller Abfall

5. Triexponentieller Abfall

6. Monoexponentieller Anstieg + Biexponentieller Abfall

7. Monoexponentieller Abfall + Biexponentieller Anstieg

8. Monoexponetieller Abfall (ohne Faltung)

9. Biexponetieller Abfall (ohne Faltung)

10. Triexponentieller Abfall (ohne Faltung)

Neben der bereits in Abschnitt 3.4.3 vorgestellten Modellfunktion 3 (Monoexponentieller Abfall + Monexponentieller Anstieg) wurde zur Auswertung der transienten Absorptionsmessungen in Kapitel 4 zusätzlich die Modellfunktion 6 (Monoexponentieller Anstieg + Biexponentieller Abfall) verwendet. Die entsprechende Modellfunktion ergibt sich dann wie folgt:

$$
\begin{aligned}
e(t) & =A \cdot e_{1}(t)+B \cdot e_{2}(t)+C \cdot e_{3}(t)+I_{0} \cdot e_{4}(t) \\
e_{1}(t) & =\exp \left(-\frac{t}{\tau_{1}}\right) ; e_{2}(t)=\exp \left(-\frac{t}{\tau_{2}}\right) ; e_{3}(t)=1-\exp \left(-\frac{t}{\tau_{3}}\right) ; e_{4}(t)=1 \\
g(t) & =\frac{1}{2 \pi \sigma^{2}} \exp \left(-\frac{\left(t-t_{0}\right)^{2}}{2 \sigma^{2}}\right) .
\end{aligned}
$$


Daraus folgt für die Fitfunktion:

$$
\begin{aligned}
f(t) & =A \cdot \int_{0}^{\infty} g(t) \cdot e_{1}(t+\tau) \mathrm{d} \tau+B \cdot \int_{0}^{\infty} g(t) \cdot e_{2}(t+\tau) \mathrm{d} \tau \\
& +C \cdot \int_{0}^{\infty} g(t) \cdot e_{3}(t+\tau) \mathrm{d} \tau+I_{0} \cdot \int_{0}^{\infty} g(t) \cdot e_{4}(t+\tau) \mathrm{d} \tau+I_{1} \\
& =A \cdot \exp \left(\frac{\sigma^{2}-2\left(t-t_{0}\right) \tau_{1}}{2 \tau_{1}^{2}}\right) \cdot \sqrt{\frac{\pi}{2}} \sigma\left(1+\operatorname{erf}\left(\frac{-\sigma^{2}+\left(t-t_{0}\right) \tau_{1}}{\sqrt{2} \sigma \tau_{1}}\right)\right) \\
& +B \cdot \exp \left(\frac{\sigma^{2}-2\left(t-t_{0}\right) \tau_{2}}{2 \tau_{2}^{2}}\right) \cdot \sqrt{\frac{\pi}{2}} \sigma\left(1+\operatorname{erf}\left(\frac{-\sigma^{2}+\left(t-t_{0}\right) \tau_{2}}{\sqrt{2} \sigma \tau_{2}}\right)\right) \\
& +C \cdot \exp \left(\frac{-\left(t-t_{0}\right)}{2 \tau_{3}^{2}}\right) \cdot \sqrt{\frac{\pi}{2}} \sigma \cdot \exp \left(\frac{t-t_{0}}{\tau_{3}}\right) \cdot\left(1+\operatorname{erf}\left(\frac{t-t_{0}}{\sqrt{2} \sigma}\right)\right) \\
& +\exp \left(\frac{\sigma^{2}}{2 \tau_{3}^{2}}\right) \cdot\left(-1+\operatorname{erf}\left(\frac{\sigma^{2}-\left(t-t_{0}\right) \tau_{3}}{\sqrt{2} \sigma \tau_{3}}\right)\right) \\
& +I_{0} \cdot \sqrt{\frac{\pi}{2}} \sigma\left(1+\operatorname{erf}\left(\frac{t-t_{0}}{\sqrt{2} \sigma}\right)\right)+I_{1},
\end{aligned}
$$

mit den Fitparametern:

- $A, B, C$ : Amplituden

- $t_{o}$ : Zeitnullpunkt

- $\tau_{1}, \tau_{2}$ : Abfallzeitkonstanten

- $\tau_{3}$ : Anstiegszeitkonstante

- $\sigma$ : halbe Halbwertsbreite der Gaußfunktion (Kreuzkorrelation)

- $I_{0}$ : Endoffset

- $I_{1}$ : Gesamtoffset. 


\section{A.2 Daten der Anpassungen}

Die folgenden Tabellen zeigen die Parameter der Anpassungen mit dem in Abschnitt 3.4.3 beschriebenen Programm an die Signale. Dabei entspricht $\tau_{1}$ der Zeitkonstante $\tau_{\text {Abfall }}$ des schnellen Signalabfalls und $\tau_{2}$ der Zeitkonstanten $\tau_{\text {Anstieg }}$ des langsamen Anstiegs mit den zugehörigen Amplituden $A$ und $B$ der Anpassungsfunktion (siehe Gleichung 3.11 auf Seite 39 ). Der Parameter $A_{\text {Anstieg }}$ entspricht der Endamplitude des Plateausignals und $\tau_{\text {cc }}$ entspricht $2 \sigma$.

\section{A.2.1 Überkritisches $\mathrm{CO}_{2}$}

\begin{tabular}{|c|c|c|c|c|c|c|c|}
\hline $\begin{array}{r}\text { Datei } \\
\mathrm{Nr} .\end{array}$ & $p /$ bar & $\tau_{\text {cc }} /$ fs & $\tau_{1} / \mathrm{fs}$ & $A \cdot 10^{-6}$ & $\tau_{2} / \mathrm{ps}$ & $B \cdot 10^{-6}$ & $\begin{array}{c}A_{\text {Anstieg }} \\
/ \Delta \text { mOD }\end{array}$ \\
\hline 303 & 150 & 170 & 180 & $\overline{44,48}$ & 27,2 & 7,22 & $\overline{2,07}$ \\
\hline 312 & 345 & 190 & 190 & 3,46 & 21,3 & 10,09 & 2,90 \\
\hline 329 & 460 & 180 & 230 & 4,97 & 18,9 & 16,88 & 4,58 \\
\hline 334 & 700 & 180 & 270 & 3,69 & 15,8 & 20,08 & 5,24 \\
\hline 335 & 750 & 180 & 280 & 3,67 & 15,0 & 20,56 & 5,32 \\
\hline 336 & 810 & 180 & 280 & 3,55 & 14,6 & 21,18 & 5,47 \\
\hline 337 & 850 & 180 & 265 & 3,84 & 14,2 & 21,48 & 5,50 \\
\hline 386 & 120 & 180 & 140 & 2,48 & 29,3 & 2,93 & 0,84 \\
\hline 388 & 117 & 180 & 150 & 1,89 & 29,1 & 2,35 & 0,71 \\
\hline 391 & 136 & 180 & 135 & 1,46 & 28,9 & 2,96 & 0,88 \\
\hline 392 & 179 & 180 & 190 & 1,35 & 23,9 & 2,97 & 0,78 \\
\hline 428 & 195 & 180 & 190 & 1,53 & 28,0 & 3,65 & 1,05 \\
\hline 441 & 382 & 180 & 170 & 5,54 & 20,3 & 14,93 & 4,04 \\
\hline 462 & 211 & 210 & 165 & 4,50 & 25,3 & 6,86 & 2,09 \\
\hline 463 & 254 & 230 & 170 & 4,29 & 23,7 & 7,22 & 2,37 \\
\hline 464 & 312 & 210 & 190 & 4,14 & 22,6 & 8,91 & 2,66 \\
\hline 465 & 358 & 220 & 165 & 4,49 & 21,5 & 9,38 & 2,94 \\
\hline 473 & 385 & 180 & 200 & 5,25 & 21,9 & 13,59 & 3,65 \\
\hline 478 & 380 & 180 & 180 & 6,84 & 21,6 & 15,89 & 4,19 \\
\hline 480 & 460 & 180 & 240 & 5,78 & 20,0 & 17,78 & 4,61 \\
\hline 481 & 515 & 170 & 200 & 6,08 & 19,2 & 19,04 & 4,80 \\
\hline 482 & 565 & 170 & 240 & 5,33 & 18,3 & 19,93 & 4,94 \\
\hline 483 & 609 & 180 & 220 & 5,41 & 17,4 & 19,35 & 5,04 \\
\hline
\end{tabular}

Tabelle A.1: Übersicht der Zeiten und Amplituden für eine zweifachexponentielle Anpassung (siehe Gleichung 3.11 auf Seite 39) der Signale von Diiodmethan in überkritischem $\mathrm{CO}_{2}$ bei $304 \mathrm{~nm}$ Anregung und $370 \mathrm{~nm}$ Abfrage und einer Temperatur von $308 \mathrm{~K}$. 


\begin{tabular}{|c|c|c|c|c|c|c|c|}
\hline $\begin{array}{c}\text { Datei } \\
\text { Nr. }\end{array}$ & $p$ / bar & $\tau_{\mathbf{c c}} / \mathrm{fs}$ & $\tau_{1} /$ fs & $A \cdot 10^{-6}$ & $\tau_{2} / \mathbf{p s}$ & $B \cdot 10^{-6}$ & $\begin{array}{r}A_{\text {Anstieg }} \\
/ \Delta \text { mOD }\end{array}$ \\
\hline 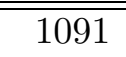 & 165 & "110 & 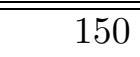 & 2,25 & "13,5 & 2,74 & (0,63 \\
\hline 1093 & 270 & 140 & 40 & 4,50 & 12,3 & 3,25 & 0,79 \\
\hline 1095 & 349 & 170 & 30 & 2,25 & 11,9 & 3,26 & 0,81 \\
\hline 1099 & 515 & 90 & 110 & 2,25 & 9,9 & 7,63 & 1,02 \\
\hline 1101 & 600 & 140 & 130 & 2,25 & 9,9 & 5,95 & 1,21 \\
\hline 1140 & 180 & 170 & 110 & 3,09 & 14,2 & 2,58 & 0,65 \\
\hline 1143 & 162 & 170 & 110 & 4,52 & 14,8 & 2,47 & 0,71 \\
\hline 1144 & 208 & 190 & 110 & 3,96 & 13,9 & 2,54 & 0,82 \\
\hline 1145 & 258 & 180 & 130 & 4,02 & 13,6 & 3,06 & 0,92 \\
\hline 1146 & 310 & 200 & 140 & 4,76 & 12,4 & 3,11 & 1,02 \\
\hline 1147 & 358 & 180 & 120 & 3,13 & 11,8 & 4,13 & 1,11 \\
\hline 1148 & 399 & 200 & 150 & 3,53 & 11,3 & 3,70 & 1,15 \\
\hline 1149 & 454 & 190 & 170 & 3,20 & 10,9 & 4,23 & 1,24 \\
\hline 1150 & 500 & 210 & 110 & 4,08 & 10,7 & 3,97 & 1,29 \\
\hline 1151 & 546 & 220 & 140 & 3,73 & 10,1 & 3,97 & 1,37 \\
\hline 1152 & 600 & 220 & 180 & 3,03 & 9,9 & 4,28 & 1,43 \\
\hline 1153 & 652 & 240 & 140 & 4,13 & 9,8 & 4,07 & 1,49 \\
\hline 1155 & 745 & 260 & 150 & 4,15 & 9,0 & 4,03 & 1,60 \\
\hline 1156 & 805 & 230 & 190 & 3,86 & 8,5 & 4,70 & 1,67 \\
\hline 1164 & 108 & 140 & 230 & 2,83 & 15,9 & 1,79 & 0,49 \\
\hline
\end{tabular}

Tabelle A.2: Übersicht der Zeiten und Amplituden für eine zweifachexponentielle Anpassung (siehe Gleichung 3.11 auf Seite 39) der Signale von Diiodmethan in überkritischem $\mathrm{CO}_{2}$ bei $304 \mathrm{~nm}$ Anregung und $405 \mathrm{~nm}$ Abfrage und einer Temperatur von $308 \mathrm{~K}$.

\begin{tabular}{|r|r|r|r|r|r|r|r|r|r|}
\hline $\begin{array}{r}\text { Datei } \\
\text { Nr. }\end{array}$ & $\begin{array}{r}\boldsymbol{p} \\
\text { / bar }\end{array}$ & $\begin{array}{r}\boldsymbol{\tau}_{\mathbf{c c}} \\
\boldsymbol{~ / ~ f s ~}\end{array}$ & $\begin{array}{r}\boldsymbol{\tau}_{1} \\
/ \mathbf{f s}\end{array}$ & $\begin{array}{r}\boldsymbol{A} \\
\mathbf{1 0}^{-\mathbf{6}}\end{array}$ & $\begin{array}{r}\boldsymbol{\tau}_{2} \\
/ \mathbf{p s}\end{array}$ & $\begin{array}{r}\boldsymbol{B} \\
\mathbf{1 0}^{-\mathbf{6}}\end{array}$ & $\begin{array}{r}\boldsymbol{\tau}_{3} \\
/ \mathbf{p s}\end{array}$ & $\begin{array}{r}\boldsymbol{C} \\
\mathbf{1 0}^{-\mathbf{6}}\end{array}$ & $\begin{array}{r}\boldsymbol{A}_{\text {Anstieg }} \\
\mathbf{\Delta} \mathbf{m O D}\end{array}$ \\
\hline \hline 1243 & 655 & 170 & 305 & 5,14 & 4,8 & 8,04 & 6,9 & $-4,13$ & 1,36 \\
1244 & 830 & 170 & 275 & 5,41 & 5,4 & 18,28 & 6,7 & $-13,72$ & 1,43 \\
1245 & 905 & 170 & 190 & 5,46 & 4,4 & 23,34 & 5,0 & $-18,31$ & 1,46 \\
1253 & 190 & 200 & 250 & 1,75 & 9,6 & 15,41 & 10,3 & $-14,52$ & 0,32 \\
1255 & 290 & 150 & 340 & 1,93 & 7,4 & 12,05 & 8,3 & $-10,46$ & 0,48 \\
1256 & 407 & 175 & 250 & 2,23 & 7,5 & 13,60 & 8,4 & $-11,82$ & 0,60 \\
1257 & 573 & 190 & 280 & 2,44 & 5,6 & 12,63 & 6,4 & $-10,08$ & 0,78 \\
1258 & 660 & 200 & 250 & 2,37 & 4,9 & 11,15 & 5,4 & $-8,53$ & 0,84 \\
1259 & 745 & 190 & 280 & 2,63 & 5,1 & 22,73 & 5,6 & $-19,59$ & 0,90 \\
\hline
\end{tabular}

Tabelle A.3: Übersicht der Zeiten und Amplituden für eine dreifachexponentielle Anpassung (siehe Gleichung A.2 auf Seite 122) der Signale von Diiodmethan in überkritischem $\mathrm{CO}_{2}$ bei $304 \mathrm{~nm}$ Anregung und $435 \mathrm{~nm}$ Abfrage und einer Temperatur von $308 \mathrm{~K}$. 


\section{A.2.2 Überkritisches $\mathrm{CHF}_{3}$}

\begin{tabular}{|r|r|r|r|r|r|r|r|}
\hline $\begin{array}{r}\text { Datei } \\
\text { Nr. }\end{array}$ & $\boldsymbol{p} /$ bar & $\boldsymbol{\tau}_{\mathbf{c c}} / \mathbf{f s}$ & $\boldsymbol{\tau}_{1} / \mathbf{f s}$ & $\boldsymbol{A} \cdot \mathbf{1 0}^{-\mathbf{6}}$ & $\boldsymbol{\tau}_{2} / \mathbf{p s}$ & $\boldsymbol{B} \cdot \mathbf{1 0}^{-\mathbf{6}}$ & $\begin{array}{r}\boldsymbol{A}_{\text {Anstieg }} \\
/ \mathbf{\Delta} \mathbf{m O D}\end{array}$ \\
\hline \hline 16027 & 175 & 170 & 90 & 0,76 & 15,7 & 3,16 & 0,76 \\
16028 & 200 & 170 & 140 & 0,34 & 15,6 & 3,24 & 0,75 \\
16029 & 300 & 170 & 150 & 0,56 & 13,7 & 4,37 & 0,94 \\
16030 & 450 & 170 & 230 & 0,38 & 11,5 & 5,88 & 1,25 \\
16031 & 600 & 170 & 250 & 0,68 & 10,6 & 6,99 & 1,49 \\
16032 & 800 & 170 & 350 & 0,49 & 9,8 & 8,12 & 1,70 \\
16033 & 900 & 170 & 610 & 0,44 & 9,0 & 8,47 & 1,75 \\
\hline
\end{tabular}

Tabelle A.4: Übersicht der Zeiten und Amplituden für eine zweifachexponentielle Anpassung (siehe Gleichung 3.11 auf Seite 39) der Signale von Diiodmethan in überkritischem $\mathrm{CHF}_{3}$ bei $315 \mathrm{~nm}$ Anregung und $410 \mathrm{~nm}$ Abfrage und einer Temperatur von $310 \mathrm{~K}$.

\section{A.2.3 Überkritisches Ethan}

\begin{tabular}{|r|r|r|r|r|r|r|r|}
\hline $\begin{array}{r}\text { Datei } \\
\text { Nr. }\end{array}$ & $\boldsymbol{p} /$ bar & $\boldsymbol{\tau}_{\mathbf{c c}} / \mathbf{f s}$ & $\boldsymbol{\tau}_{1} / \mathbf{f s}$ & $\boldsymbol{A} \cdot \mathbf{1 0}^{-\mathbf{6}}$ & $\boldsymbol{\tau}_{2} / \mathbf{p s}$ & $\boldsymbol{B} \cdot \mathbf{1 0}^{-\mathbf{6}}$ & $\begin{array}{r}\boldsymbol{A}_{\text {Anstieg }} \\
/ \mathbf{A} \text { mOD }\end{array}$ \\
\hline \hline 2059 & 250 & 170 & 300 & 1,33 & 13,8 & 2,08 & 0,57 \\
2060 & 350 & 170 & 300 & 1,41 & 13,0 & 2,29 & 0,67 \\
2074 & 450 & 170 & 300 & 1,08 & 10,8 & 2,49 & 0,67 \\
2077 & 550 & 170 & 300 & 1,03 & 10,0 & 2,94 & 0,77 \\
2078 & 650 & 170 & 300 & 1,28 & 9,1 & 3,41 & 0,89 \\
2079 & 750 & 170 & 300 & 1,12 & 8,7 & 3,89 & 1,02 \\
2080 & 850 & 170 & 300 & 1,40 & 7,3 & 4,41 & 1,09 \\
2081 & 950 & 170 & 300 & 1,21 & 7,6 & 4,85 & 1,18 \\
\hline
\end{tabular}

Tabelle A.5: Übersicht der Zeiten und Amplituden für eine zweifachexponentielle Anpassung (siehe Gleichung 3.11 auf Seite 39) der Signale von Diiodmethan in überkritischem Ethan bei $315 \mathrm{~nm}$ Anregung und $405 \mathrm{~nm}$ Abfrage und einer Temperatur von $310 \mathrm{~K}$. 


\section{A.3 Thermodynamische Daten der verwendeten Lösungsmittel}

\section{A.3.1 Überkritisches $\mathrm{CO}_{2}$}

\section{Druck-/Dichtebeziehung}

Für die Umrechnung der Druck- in eine Dichteskala wurden Druck-/Dichtedaten aus dem NIST Chemistry WebBook [140] entnommen und mit einer zweifachexponentiellen Funktion angepasst (siehe Abbildung A.1). Mit dieser angepassten Funktion wurden die gemessen Druckdaten bei fester Temperatur umgerechnet. Die Parameter der Anpassung lauten wie folgt:

$$
\begin{aligned}
& \rho=p_{0}+A_{1} \cdot\left(1-\exp \left(-\frac{p}{p_{1}}\right)\right)+A_{2} \cdot\left(1-\exp \left(-\frac{p}{p_{2}}\right)\right) \\
& \text { mit: } \\
& p_{0}=1,78(69) \\
& A_{1}=9,714(29) \quad p_{1}=621(13) \\
& A_{2}=15,68(64) \quad p_{2}=55,6(15) \\
& \text { und } R^{2}=0,99985 \text {. }
\end{aligned}
$$

\section{Druck-/Viskositätsbeziehung}

Für die Umrechnung der Druck- in eine Viskositätskala wurden ebenfalls experimentelle Daten aus dem NIST Chemistry WebBook [140] entnommen und mit einer zweifachexponentiellen Funktion angepasst (siehe Abbildung A.2). Dazu wurden folgende Parameter verwendet:

$$
\begin{array}{ll}
\eta=p_{0}+A_{1} \cdot\left(1-\exp \left(-\frac{p}{p_{1}}\right)\right)+A_{2} \cdot\left(1-\exp \left(-\frac{p}{p_{2}}\right)\right) \\
\text { mit: } & \\
p_{0}=-0,0066(23) & p_{1}=2220(69) \\
A_{1}=0,3056(66) & p_{2}=70,0(19) \\
A_{2}=0,0679(20) &
\end{array}
$$




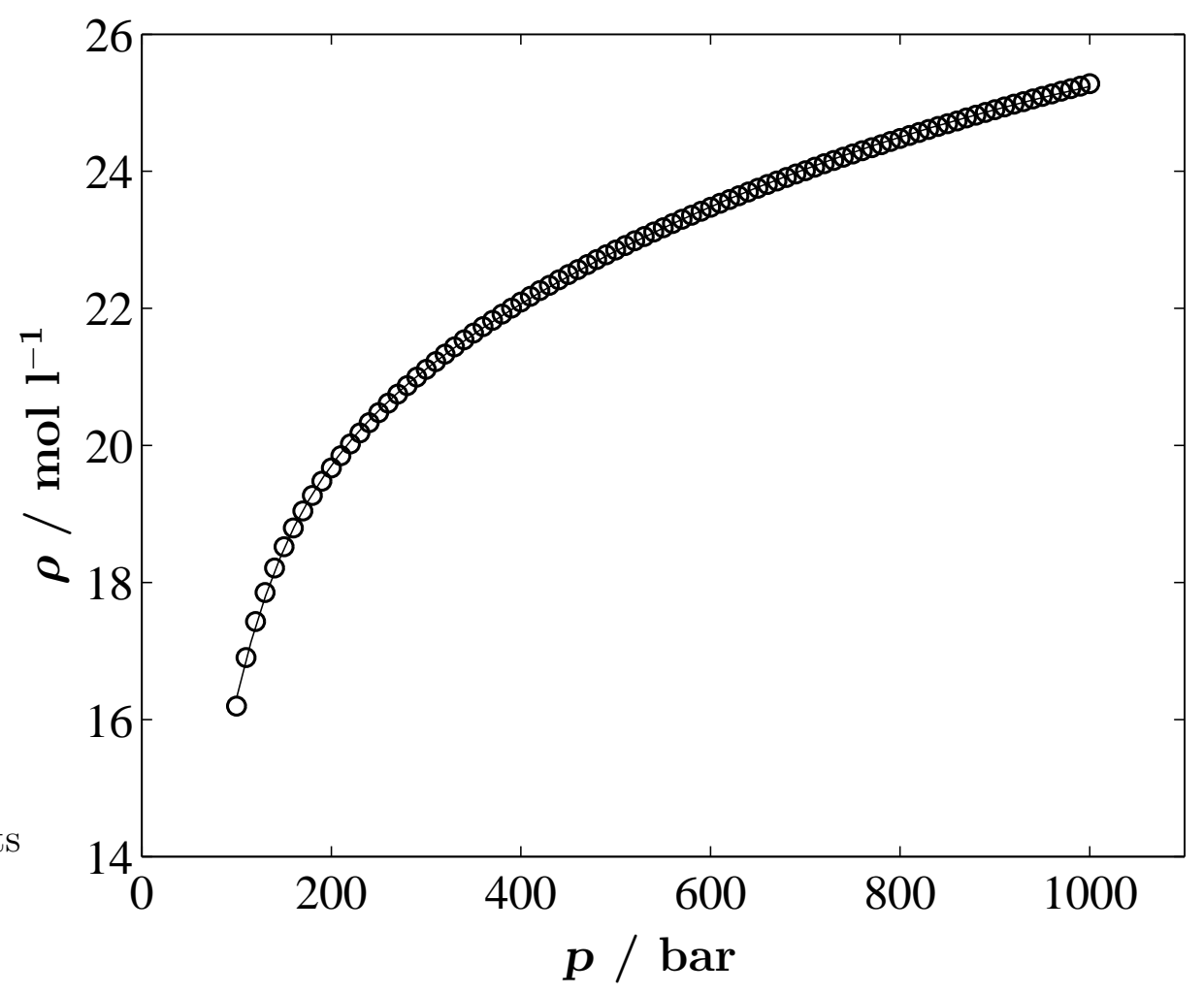

Abbildung A.1: Druck-/Dichtebeziehung für $\mathrm{CO}_{2}$ bei $308 \mathrm{~K}$ aus [140].

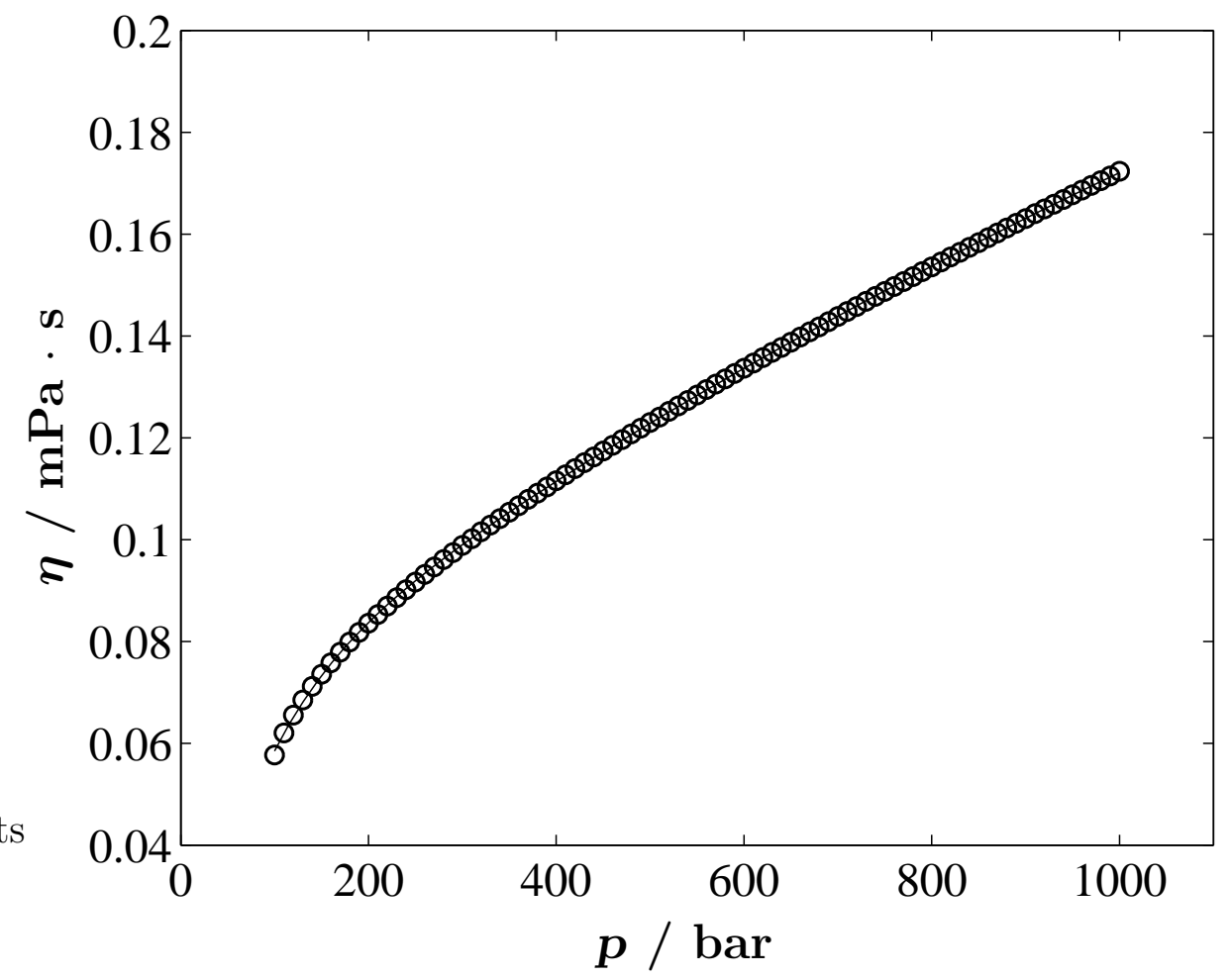

Abbildung A.2: Druck-/Viskositätsbeziehung für $\mathrm{CO}_{2}$ bei $308 \mathrm{~K}$ aus [140]. 


\section{Berechnung des Clausius-Mosotti-Faktors}

Für die Berechnung des Clausius-Mosotti-Faktors bei verschiedenen Dichten wurde die Polynomische Näherung aus [180] verwendet. Dannach gilt für $f(n)$ in überkritischem $\mathrm{CO}_{2}$ :

$$
f(n)=\frac{n^{2}-1}{n^{2}+2}=0,07016 \rho_{r}+1,412 \cdot 10^{-4} \rho_{r}^{2}-3,171 \cdot 10^{-4} \rho_{r}^{3} .
$$

\section{A.3.2 Überkritisches $\mathrm{CHF}_{3}$}

\section{Druck-/Dichtebeziehung}

Für die Umrechnung der Druck- in eine Dichteskala bei $\mathrm{CHF}_{3}$ wurden vergleichbar dem Verfahren bei $\mathrm{CO}_{2}$ (siehe A.3.1), die Druck-/Dichtedaten mit einer zweifachexponentiellen Funktion angepasst (siehe Abbildung A.1). Mit dieser angepassten Funktion konnten die gemessenen Druckdaten bei fester Temperatur umgerechnet werden. Für die Anpassung der experimentellen Daten wurde folgende Gleichung benutzt:

$$
\begin{array}{ll}
\rho=p_{0}+A_{1} \cdot\left(1-\exp \left(-\frac{p}{p_{1}}\right)\right)+A_{2} \cdot\left(1-\exp \left(-\frac{p}{p_{2}}\right)\right) \\
\text { mit: } & \\
p_{0}=5,250(70) & p_{1}=645,1(45) \\
A_{1}=7,5564(75) & p_{2}=64,56(49) \\
A_{2}=7,870(57) &
\end{array}
$$

Die entsprechenden $p(\rho)$-Daten wurden gemäß [188] an experimentelle Daten angepasst. Zur Berechnung der in der Abbildung A.3 verwendeten Daten wurde folgende Anpassung verwendet:

$$
\begin{aligned}
p(\rho) & =R T \rho+\left(A_{1} R T+A_{2}+\frac{A_{3}}{T}+\frac{A_{4}}{T^{2}}+\frac{A_{5}}{T^{4}}\right) \rho^{2} \\
& +\left(A_{6} R T+A_{7}\right) \rho^{3}+A_{8} T \rho^{4}+\left(\frac{A_{10}}{T^{3}}+\frac{A_{11}}{T^{4}}\right) \rho^{3} \cdot \exp \left(A_{16} \rho^{2}\right) \\
& +\left(\frac{A_{12}}{T^{2}}+\frac{A_{13}}{T^{3}}+\frac{A_{14}}{T^{4}}\right) \rho^{5} \cdot \exp \left(A_{16} \rho^{2}\right)+A_{15} \rho^{6}
\end{aligned}
$$




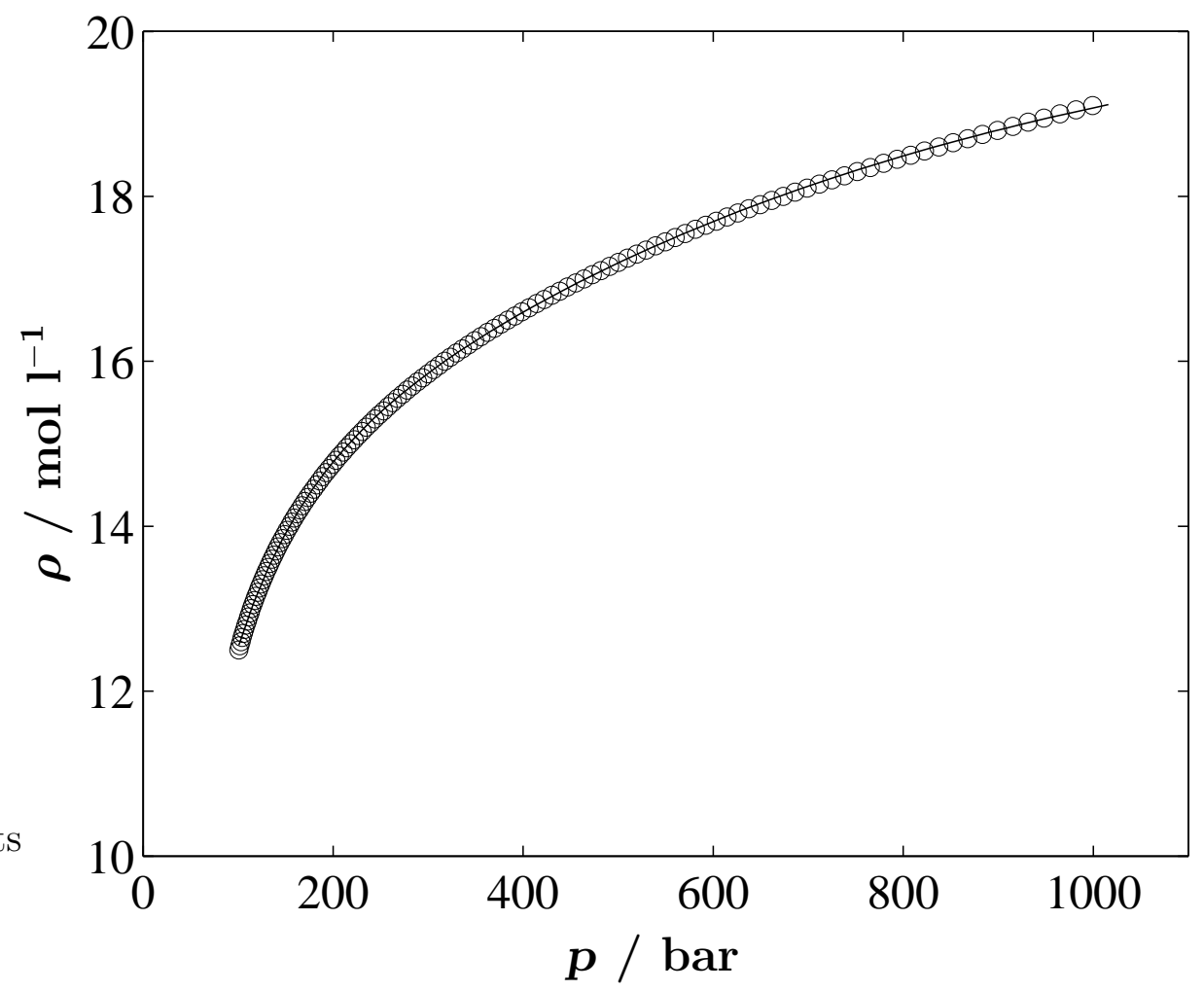

Abbildung A.3: Druck-/Dichtebeziehung für $\mathrm{CHF}_{3}$ bei $310 \mathrm{~K}$ gemäß [188].

mit folgenden Anpassungsparametern

$$
\begin{array}{ll}
A_{1}=0,045457 & A_{2}=6,9009 \\
A_{3}=-5684,3 & A_{4}=5,8935 \cdot 10^{5} \\
A_{5}=-2,6234 \cdot 10^{9} & A_{6}=-6,9026 \cdot 10^{-3} \\
A_{7}=0,21356 \text { (anderes Vorzeichen als in [188]) } & \\
A_{8}=2,2642 \cdot 10^{-5} & A_{9}=-33930 \\
A_{10}=2,9711 \cdot 10^{7} & A_{11}=-3,4292 \cdot 10^{9} \\
A_{12}=47,361 & A_{13}=-64699 \\
A_{14}=7,8646 \cdot 10^{6} & A_{15}=2,0224 \cdot 10^{-5} \\
A_{16} & =-0,0040 .
\end{array}
$$




\section{Berechnung des Clausius-Mosotti-Faktors}

Für die Berechnung des Clausius-Mosotti-Faktors bei verschiedenen Dichten wurde die Polynomische Näherung aus [180] verwendet. Dannach gilt für $f(n)$ in überkritischem $\mathrm{CHF}_{3}$ :

$$
f(n)=\frac{n^{2}-1}{n^{2}+2}=0,05374 \rho_{r}-3,136 \cdot 10^{-4} \rho_{r}^{2}-1,534 \cdot 10^{-4} \rho_{r}^{3} .
$$

\section{A.3.3 Überkritisches Ethan}

\section{Druck-/Dichtebeziehung}

Die Umrechnung der Druck- in eine Dichteskala erfolgte genau wie bei $\mathrm{CO}_{2}$ und $\mathrm{CHF}_{3}$ über eine zweifachexponentielle Anpassung der Druck-/Dichte Daten aus [140]. Die Daten und die Parameter der Anpassung sind Abbildung A.4 zu entnehmen. Leider waren die Daten nur bis 700 bar verfügbar, jedoch wurde die Anpassung mit den im Folgenden angegebenen Parametern bis 1000 bar weiter extrapoliert:

$$
\begin{array}{ll}
\rho=p_{0}+A_{1} \cdot\left(1-\exp \left(-\frac{p}{p_{1}}\right)\right)+A_{2} \cdot\left(1-\exp \left(-\frac{p}{p_{2}}\right)\right) \\
\text { mit: } & \\
p_{0}=2,08(29) & p_{1}=444,2(65) \\
A_{1}=6,208(13) & p_{2}=39,35(74) \\
A_{2}=9,25(25) &
\end{array}
$$

\section{Berechnung des Clausius-Mosotti-Faktors}

Für die Berechnung des Clausius-Mosotti-Faktors bei verschiedenen Dichten wurde die Polynomische Näherung aus [180] verwendet. Danach gilt für $f(n)$ in überkritischem Ethan:

$$
f(n)=\frac{n^{2}-1}{n^{2}+2}=0,07741 \rho_{r}+1,097 \cdot 10^{-3} \rho_{r}^{2}-5,427 \cdot 10^{-4} \rho_{r}^{3}
$$




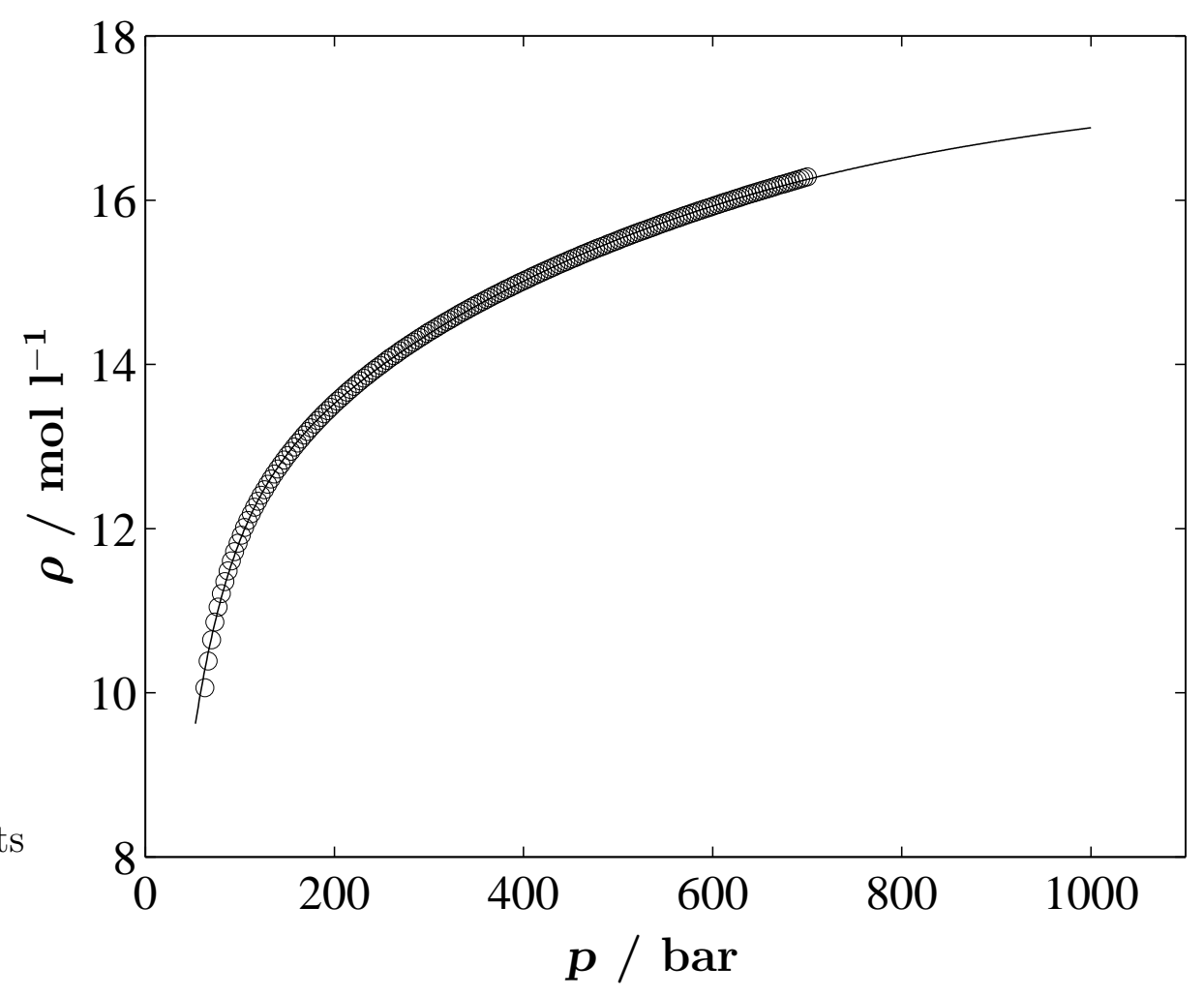

Abbildung A.4: Druck-/Dichtebeziehung für Ethan bei $310 \mathrm{~K}$ aus [140].

\section{A.3.4 Überkritisches Xenon}

\section{Druck-/Dichtebeziehung}

Für die Umrechnung der Druck- in eine Dichteskala von überkritischem Xenon wurde, wie bereits bei $\mathrm{CO}_{2}$ gezeigt (siehe Abschitt A.3.1), eine zweifachexponentielle Anpassung an experimentelle Druck-/Dichtedaten aus [140] verwendet. Die Funktion und die verwendeten Parameter für die Anpassung an die, in Abbildung A.5 dargestellten, Daten lauten wie folgt:

$$
\rho=p_{0}+A_{1} \cdot\left(1-\exp \left(-\frac{p}{p_{1}}\right)\right)+A_{2} \cdot\left(1-\exp \left(-\frac{p}{p_{2}}\right)\right)
$$

mit:

$$
\begin{array}{ll}
p_{0}=-15,4(14) & \\
A_{1}=8,904(28) & p_{1}=532,8(92) \\
A_{2}=28,3(13) & p_{2}=41,91(98)
\end{array}
$$

und $R^{2}=0,99958$ 


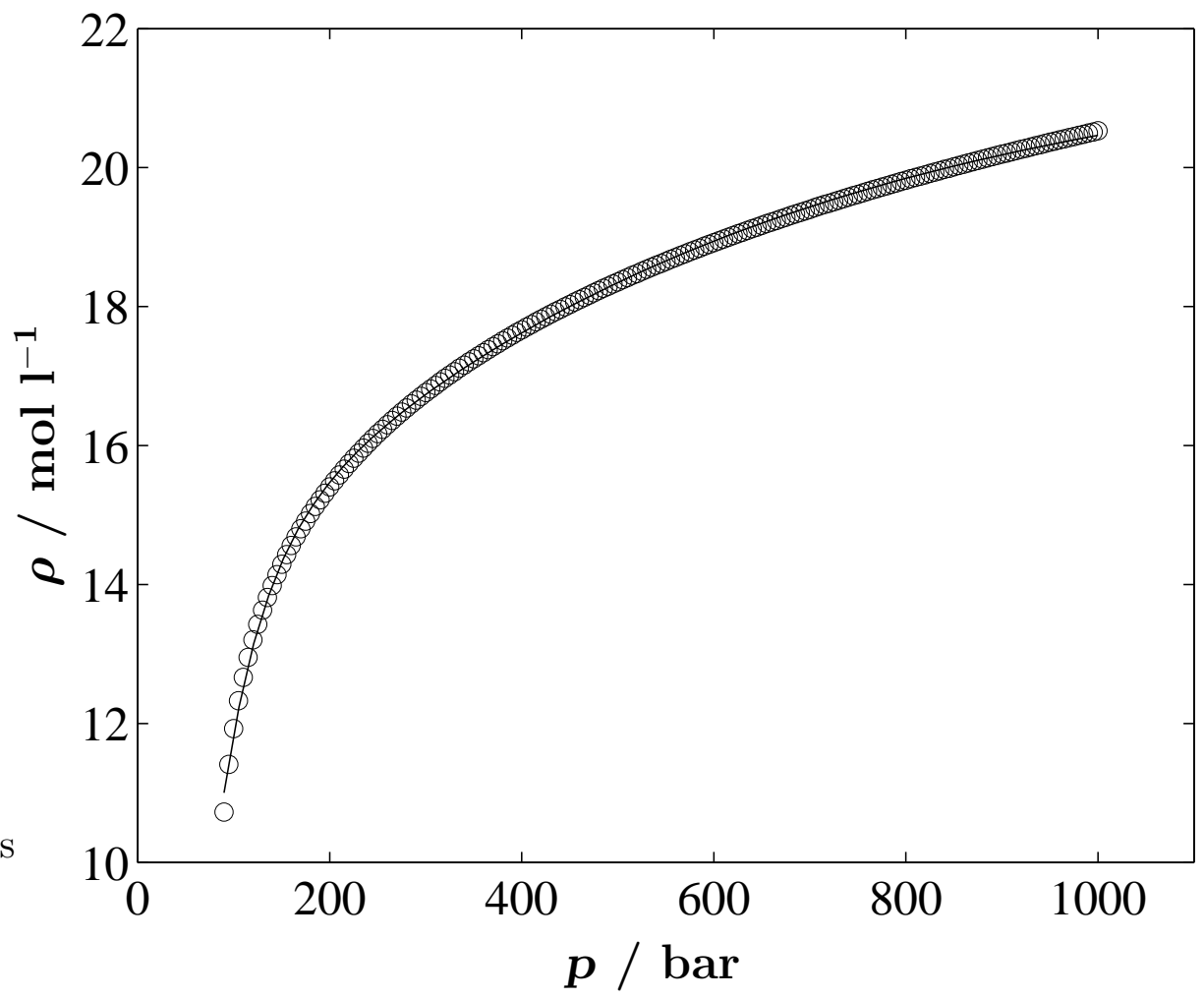

Abbildung A.5: Druck-/Dichtebeziehung für Xenon bei $308 \mathrm{~K}$ aus [140]. 


\section{Abbildungsverzeichnis}

$2.1 \mathrm{CH}_{2} \mathrm{I}_{2}$-Molekül. . . . . . . . . . . . . . . . . . . . . 9

2.2 Schematisches Energiediagramm des Diiodmethans. . . . . . . . . . . 11

2.3 Vergleich der Absorptionsspektren von $\mathrm{CH}_{2} \mathrm{I}_{2}$ [62] und $\mathrm{CH}_{2} \mathrm{I}$ [74] in der Gasphase. . . . . . . . . . . . . . . . . . . 12

2.4 Absorption der Photoprodukte (Isomer) in einer $\mathrm{N}_{2}$-Matrix nach UVAnregung [33]. . . . . . . . . . . . . . . . . . . . . 16

$2.5 \mathrm{CH}_{2} \mathrm{I}-\mathrm{I}-\mathrm{Molekül} \ldots \ldots \ldots \ldots \ldots \ldots$

3.1 Das verwendete Lasersystem im Überblick. . . . . . . . . . . . . . . . . 24

3.2 Prinzip des Faserlasers $[112,118] \ldots \ldots \ldots \ldots \ldots \ldots \ldots$

3.3 Verstärkungsprofil des Seedpulses im Resonator des Ti:Saphir-Verstärkers. 27

3.4 Autokorrelationsmessung der Pulse des CPA-2001. . . . . . . . . . 28

3.5 Schematischer Aufbau des verwendeten NOPA [113]. . . . . . . . . . . 29

3.6 Schematische Darstellung des verwendeten Pump/Probe-Interferometers. 33

3.7 Schematische Darstellung der elektronischen Steuerung des Experimentes. 37

3.8 Spektrum der Anregungspulse bei einer Zentralwellenlänge von $304 \mathrm{~nm} . \quad 40$

3.9 Beispielspektren der Abfragepulse bei verschiedenen Zentralwellenlängen. 41

3.10 Transiente Absorption bei $310 \mathrm{~nm}$ nach Anregung mit $266 \mathrm{~nm}$ in Propylencarbonat. . . . . . . . . . . . . . . . . . 42

3.11 Gesamtansicht der verwendeten Hochdruckmesszelle. . . . . . . . . . . 43

3.12 Schnittansicht der verwendeten Hochdruckmesszelle. . . . . . . . . . . . 44

3.13 Schematische Ansicht des verwendeten Hochdrucksystems. . . . . . . . 45

4.1 Stationäre Absorptionsspektren von $\mathrm{CH}_{2} \mathrm{I}_{2}$ in verschiedenen organischen Lösungsmitteln. . . . . . . . . . . . . . . . . . . 5 50

4.2 Stationäre Absorptionsspektren von $\mathrm{CH}_{2} \mathrm{I}_{2}$ in verschiedenen überkritischen Lösungsmitteln. . . . . . . . . . . . . . . . . . . . . 51

4.3 Transiente Absorption von $\mathrm{CH}_{2} \mathrm{I}_{2}$ in Acetonitril. . . . . . . . . . . . 52

4.4 Erstes Signal in der Hochdruckzelle. . . . . . . . . . . . . . . . . 54

4.5 Typisches Signal in überkritischem $\mathrm{CO}_{2} \ldots \ldots \ldots \ldots \ldots$

4.6 Transiente Messungen in überkritischem $\mathrm{CO}_{2}$ bei verschiedenen Probe-

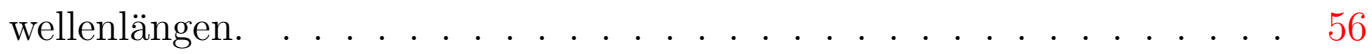


4.7 Transientes Absorptionsspektrum in überkritischem $\mathrm{CO}_{2}$ zu langen Zeiten. . . . . . . . . . . . . . . . . . . 57

4.8 Auf Fläche normiertes, transientes Absorptionsspektrum in überkritischem $\mathrm{CO}_{2}$ zu langen Zeiten. . . . . . . . . . . . . . 58

4.9 Transientes Absorptionsspektrum in überkritischem $\mathrm{CO}_{2}$ zu kurzen Zeiten. . . . . . . . . . . . . . . . . . . . 59

4.10 Auf Fläche normiertes, transientes Absorptionsspektrum in überkritischem $\mathrm{CO}_{2}$ zu kurzen Zeiten. . . . . . . . . . . . . . . . . . 60

4.11 Druckabhängige Messungen in überkritischem $\mathrm{CO}_{2} \ldots \ldots$. . . . . . . 61

4.12 Druckabhängigkeit der Zeitkonstanten des Anstiegs $\tau_{\text {Anstieg }}$ und der Amplitude $A_{\text {Anstieg }}$ in überkritischem $\mathrm{CO}_{2} \ldots \ldots \ldots \ldots \ldots$

4.13 Dichteabhängigkeit der Zeitkonstanten des Anstiegs $\tau_{\text {Anstieg }}$ und der relativen Amplitude $A_{\text {Anstieg }}$ in überkritischem $\mathrm{CO}_{2} \ldots \ldots \ldots$. . . . 64

4.14 Druck- bzw. Dichteabhängigkeit der Geschwindigkeitskonstanten des

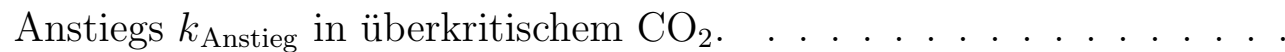

4.15 Dichteabhängigkeit der Geschwindigkeitskonstanten $k_{\text {Anstieg }}$ und der normierten Amplitude $A_{\text {Anstieg,norm }}$ des Anstiegs in überkritischem $\mathrm{CO}_{2}$ bei $304 \mathrm{~nm}$ Anregung verschiedenen Probewellenlängen. . . . . . . . .

4.16 Dichteabhängigkeit der relativen Geschwindigkeitskonstanten $k_{\text {Anstieg,rel }}$ und der relativen normierten Amplituden $A_{\text {Anstieg,norm,rel }}$ des Anstiegs in überkritischem $\mathrm{CO}_{2}$ bei $304 \mathrm{~nm}$ Anregung und verschiedenen Probewellenlängen. . . . . . . . . . . . . . . . . . .

4.17 Druckabhängigkeit der Zeitkonstanten des schnellen Abfalls $\tau_{\text {Abfall }}$ in überkritischem $\mathrm{CO}_{2} \ldots \ldots \ldots \ldots \ldots \ldots \ldots \ldots$

4.18 Auf Fläche normiertes, transientes Absorptionsspektrum in überkritischem $\mathrm{CO}_{2}$ bei verschiedenen Drücken. . . . . . . . . . . . 70

4.19 Beispiel einer Anisotropiemessung in überkritischem $\mathrm{CO}_{2} \ldots \ldots$. . . . 71

4.20 Dichteabhängigkeit der Orientierungsrelaxationszeiten $\tau_{\text {or }}$ aus Anisotropiemessungen in überkritischem $\mathrm{CO}_{2} . \ldots \ldots \ldots$. . . . . . 71

4.21 Typisches Signal in überkritischem $\mathrm{CHF}_{3} \ldots \ldots \ldots \ldots$. . . . . . 72

4.22 Transientes Absorptionsspektrum in überkritischem $\mathrm{CHF}_{3}$ zu langen Zeiten. . . . . . . . . . . . . . . . . . . 74

4.23 Auf Fläche normiertes, transientes Absorptionsspektrum in überkritischem $\mathrm{CHF}_{3}$ zu langen Zeiten. . . . . . . . . . . . . . . . . . . 74

4.24 Druckabhängigkeit der Zeitkonstanten des Anstiegs $\tau_{\text {Anstieg }}$ und der Amplitude $A_{\text {Anstieg }}$ in überkritischem $\mathrm{CHF}_{3} . \ldots \ldots \ldots \ldots$

4.25 Druck- bzw. Dichteabhängigkeit der Geschwindigkeitskonstanten des Anstiegs $k_{\text {Anstieg }}$ und der Amplitude $A_{\text {Anstieg }}$ in überkritischem $\mathrm{CHF}_{3} . \quad$. 76

4.26 Typisches Signal in überkritischem Ethan. . . . . . . . . . . . . . . . 77

4.27 Transientes Absorptionsspektrum in überkritischem Ethan zu langen Zeiten. . . . . . . . . . . . . . . . . . . 79 
4.28 Auf Fläche normiertes, transientes Absorptionsspektrum in überkritischem Ethan zu langen Zeiten. . . . . . . . . . . . . . . . . . . 79

4.29 Druckabhängigkeit der Zeitkonstanten des Anstiegs $\tau_{\text {Anstieg }}$ und der Amplitude $A_{\text {Anstieg }}$ in überkritischem Ethan. . . . . . . . . . . . . 80

4.30 Druck- bzw. Dichteabhängigkeit der Geschwindigkeitskonstanten des Anstiegs $k_{\text {Anstieg }}$ und der Amplitude $A_{\text {Anstieg }}$ in überkritischem Ethan. . 81

4.31 Typisches Signal in überkritischem Xenon. . . . . . . . . . . . . . . 82

4.32 Transientes Signal in überkritischem Xenon bei $310 \mathrm{~nm}$ Anregung und $425 \mathrm{~nm}$ Abfrage. . . . . . . . . . . . . . . . . . . 83

4.33 Transientes Absorptionsspektrum in überkritischem Xenon zu langen Zeiten. . . . . . . . . . . . . . . . . . . 85

4.34 Auf Fläche normiertes, transientes Absorptionsspektrum in überkritischem Xenon zu langen Zeiten. . . . . . . . . . . . . . . . . . . 85

5.1 Typisches Signal im Bereich kurzer Verzögerungszeiten. . . . . . . . . . 88

5.2 Kohärente Lösungsmittelantwort in überkritischem Xenon. . . . . . . . 89

5.3 Viskositätsabhängigkeit der Zeitkonstanten des schnellen Abfalls $\tau_{\text {Abfall }}$ in überkritischem $\mathrm{CO}_{2} \ldots \ldots \ldots \ldots \ldots$. . . . . . . . . 91

5.4 Extrapolation der aus Stoßwellendaten bestimmten Korrelation des Extinktionskoeffizienten $\varepsilon_{400 \mathrm{~nm}}$ mit der Überschussenergie des $\mathrm{CH}_{2} \mathrm{I}_{2}$ [88].

5.5 Viskositätsabhängigkeit der Rotationsrelaxationszeiten $\tau_{\text {rot }}$ aus Anisotropiemessungen in überkritischem $\mathrm{CO}_{2} . \ldots \ldots \ldots$. . . . . . 97

5.6 Aus den normierten Spektren ermittelte Abklingzeiten der spektralen Entwicklung $\tau_{\text {se }}$ aus den normierten Extinktionen bei verschiedenen Wellenlängen in den verwendeten Lösungsmitteln. . . . . . . . . . . 102

5.7 Abhängigkeit der Endamplitude $A_{\text {Anstieg }}$ des Signals von der reduzierten Dichte in verschiedenen überkritischen Lösungsmitteln. . . . . . . . . . 105

5.8 Abhängigkeit der normierten Endamplitude $A_{\text {Anstieg,norm }}$ des Signals von der reduzierten Dichte in verschiedenen überkritischen Lösungsmitteln. 106

5.9 Abhängigkeit der Geschwindigkeitskonstanten $k_{\text {Anstieg }}$ des Anstiegs von dem Clausius-Mosotti-Faktor in verschiedenen überkritischen Lösungsmitteln. . . . . . . . . . . . . . . . . . . . . . . . . . 108

5.10 Abhängigkeit der Geschwindigkeitskonstanten $k_{\text {Anstieg }}$ des Anstiegs von der reduzierten Dichte in verschiedenen überkritischen Lösungsmitteln. 110

A.1 Druck-/Dichtebeziehung für $\mathrm{CO}_{2}$ bei $308 \mathrm{~K}$ aus [140] . . . . . . . . 127

A.2 Druck-/Viskositätsbeziehung für $\mathrm{CO}_{2}$ bei $308 \mathrm{~K}$ aus [140]. . . . . . . . 127

A.3 Druck-/Dichtebeziehung für $\mathrm{CHF}_{3}$ bei $310 \mathrm{~K}$ gemäß [188]. . . . . . . . 129

A.4 Druck-/Dichtebeziehung für Ethan bei $310 \mathrm{~K}$ aus [140]. . . . . . . . . 131

A.5 Druck-/Dichtebeziehung für Xenon bei $308 \mathrm{~K}$ aus [140] . . . . . . . . . 132 



\section{Tabellenverzeichnis}

2.1 Normalmoden der Schwingungen in $\mathrm{CH}_{2} \mathrm{I}_{2}$ [58]. . . . . . . . . . . . . 14

2.2 Quantenchemisch berechnete Normalmoden der Schwingungen und Gleichgewichtsgeometrie von $\mathrm{CH}_{2} \mathrm{I}-\mathrm{I}$. . . . . . . . . . . . . . 21

3.1 Herkunft und Reinheit der verwendeten Chemikalien. . . . . . . . . . 47

A.1 Übersicht der Zeiten und Amplituden für eine zweifachexponentielle Anpassung der Signale von Diiodmethan in überkritischem $\mathrm{CO}_{2}$ bei $370 \mathrm{~nm}$ Abfrage. . . . . . . . . . . . . . . . . . . . 123

A.2 Übersicht der Zeiten und Amplituden für eine zweifachexponentielle Anpassung der Signale von Diiodmethan in überkritischem $\mathrm{CO}_{2}$ bei $405 \mathrm{~nm}$ Abfrage. . . . . . . . . . . . . . . . . . . 124

A.3 Übersicht der Zeiten und Amplituden für eine zweifachexponentielle Anpassung der Signale von Diiodmethan in überkritischem $\mathrm{CO}_{2}$ bei $435 \mathrm{~nm}$ Abfrage. . . . . . . . . . . . . . . . . . . . . 124

A.4 Übersicht der Zeiten und Amplituden für eine zweifachexponentielle Anpassung der Signale von Diiodmethan in überkritischem $\mathrm{CHF}_{3}$. . . . 125

A.5 Übersicht der Zeiten und Amplituden für eine zweifachexponentielle Anpassung der Signale von Diiodmethan in überkritischem Ethan. . . . 125 



\section{Literaturverzeichnis}

[1] R. Gilbert und S. Smith, Theory of Unimolecular and Recombination Reactions, Blackwell Scientific Publications, Oxford (1990). 3

[2] T. Baer und W. L. Hase, Unimolecular Reaction Dynamics: Theory and Experiments, Oxford University Press, New York (1996). 3

[3] J. Steinfeld, J. Francisco und W. Hase, Chemical Kinetics and Dynamics, Prentice Hall, Englewood Cliffs, 2. Aufl. (1998). 3

[4] J. T. Hynes, Ann. Rev. Phys. Chem., 36 (1985) 573. 3

[5] J. Franck und E. Rabinowitsch, Trans. Faraday Soc., 30 (1934) 120. 3

[6] J. Franck, Trans. Faraday Soc., 21 (1925) 536. 3

[7] J. Zimmerman und R. M. Noyes, J. Chem. Phys., 18 (1950) 658. 3

[8] F. W. Lampe und R. M. Noyes, J. Am. Chem. Soc., 76 (1954) 2140. 3

[9] L. F. Meadows und R. M. Noyes, J. Am. Chem. Soc., 82 (1960) 1872. 3

[10] D. Booth und R. M. Noyes, J. Am. Chem. Soc., 82 (1960) 1868. 3

[11] K. Luther und J. Troe, Chem. Phys. Lett., 24 (1974) 85. 4

[12] B. Otto, J. Schroeder und J. Troe, J. Chem. Phys., 81 (1984) 202. 4, 90

[13] J. Schroeder und J. Troe, Ann. Rev. Phys. Chem., 38 (1987) 163. 4, 6

[14] A. L. Harris, J. K. Brown und C. B. Harris, Ann. Rev. Phys. Chem., 39 (1988) 341. 4,89

[15] R. Zadoyan, Z. Li, P. Ashjian, C. C. Martens und V. A. Apkarian, Chem. Phys. Lett., 218 (1994) 504. 4

[16] J.-K. Wang, Q. Liu und A. H. Zewail, J. Phys. Chem., 99 (1995) 11309. 4

[17] C. Lienau und A. H. Zewail, J. Phys. Chem., 100 (1996) 18629. 4 
[18] C. Wan, M. Gupta, J. S. Baskin, Z. H. Kim und A. H. Zewail, J. Chem. Phys., 106 (1997) 4353. 4

[19] D. L. Bunker und B. S. Jacobson, J. Am. Chem. Soc., 94 (1972) 1843. 5

[20] J. N. Murrel, A. J. Stace und R. Dammel, J. Chem. Soc. Faraday Trans. 2, 74 (1978) 1532. 5

[21] J. P. Bergsma, J. R. Reimers und K. R. Wilson, J. Chem. Phys., 85 (1986) 5625. 5

[22] J. K. Brown, C. B. Harris und J. C. Tully, J. Chem. Phys., 89 (1988) 6687. 5

[23] M. Ben-Nun und R. D. Levine, J. Phys. Chem., 97 (1993) 2334. 5

[24] W. Wang, K. A. Nelson, L. Xiao und D. F. Coker, J. Chem. Phys., 101 (1994) 9663. 5

[25] M. Ben-Nun, R. D. Levine, D. M. Jonas und G. R. Fleming, Chem. Phys. Lett., 254 (1995) 629. 5

[26] V. S. Batista und D. F. Coker, J. Chem. Phys., 105 (1996) 4033. 5

[27] H. Ooe, Y. Kimura, M. Terazima und N. Hirota, J. Phys. Chem. A, 103 (1999) 7730. 5

[28] D. Schwarzer, J. Schroeder und C. Schröder, Z. Phys. Chem., 215 (2001) 183. 5, 101, 107

[29] R. A. Gregory und D. W. G. Style, Trans. Faraday Soc., 32 (1936) 724. 5, 9

[30] M. Kawasaki, S. J. Lee und R. Bersohn, J. Chem. Phys., 63 (1975) 809. 5, 9, $10,11,13,87$

[31] G. E. Busch und K. R. Wilson, J. Chem. Phys., 56 (1972) 3626. 5

[32] H. Mohan, K. N. Rao und R. M. Iyer, Radiat. Phys. Chem., 23 (1984) 505. 6, $15,16,17$

[33] G. Maier, H. P. Reisenauer, J. Hu, L. J. Schaad und B. A. Hess Jr., J. Am. Chem. Soc., 112 (1990) 5117. 6, 15, 16, 18, 19, 57, 120, 133

[34] B. J. Schwartz, J. C. King, J. Z. Zhang und C. B. Harris, Chem. Phys. Lett., 203 (1993) 503. 6, 17, 18, 20, 53

[35] K.-i. Saitow, Y. Naitoh, K. Tominaga und K. Yoshihara, Chem. Phys. Lett., 262 (1996) 621. 6, 17, 19, 20, 53, 90, 99, 118

[36] A. N. Tarnovsky, J.-L. Alvarez, A. P. Yartsev, V. Sundström und E. Åkesson, Chem. Phys. Lett., 312 (1999) 121. 6, 19, 20, 53, 57, 58, 59, 87, 89, 94, 100, $107,117,118,120$ 
[37] W. M. Kwock, C. Ma, A. W. Parker, D. Phillips, M. Towrie, P. Matousek und D. L. Phillips, J. Chem. Phys., 113 (2000) 7471. 6, 21, 94

[38] C. L. Thomsen, P. J. Reid und S. R. Keiding, J. Am. Chem. Soc., 122 (2000) 12795. 6

[39] J. Larsen, D. Madsen, J.-A. Poulsen, T. D. Poulsen, S. R. Keiding und J. Thøgersen, J. Chem. Phys., 116 (2002) 7997. 6

[40] X. Zheng und D. L. Phillips, J. Chem. Phys., 324 (2000) 175. 6

[41] M. Wall, A. N. Tarnovsky, T. Pascher, V. Sundström und E. Åkesson, J. Phys. Chem. A, 107 (2003) 211. 6

[42] X. Zheng, W. M. Kwock und D. L. Phillips, J. Phys. Chem. A, 104 (2000) 10464. 6

[43] A. N. Tarnovsky, M. Wall, M. Rasmusson, T. Pascher und E. Åkesson, J. Chin. Chem. Soc.-Taip., 47 (2000) 769. 6, 120

[44] W. M. Kwock, C. Ma, A. W. Parker, D. Phillips, M. Towrie, P. Matousek, X. Zheng und D. L. Phillips, J. Chem. Phys., 114 (2001) 7536. 6, 99, 120

[45] X. Zheng und D. L. Phillips, J. Chem. Phys., 113 (2000) 3194. 6

[46] W. M. Kwock, C. Ma, D. Phillips, A. W. Parker, M. Towrie, P. Matousek und D. L. Phillips, Chem. Phys. Lett., 341 (2001) 292. 6, 120

[47] A. N. Tarnovsky, M. Wall, M. Gustafsson, N. Lascoux, V. Sundström und E. Åkesson, J. Phys. Chem. A, 106 (2002) 5999. 6

[48] X. Zheng, C. W. Lee, Y.-L. Li, W.-H. Fang und D. L. Phillips, J. Chem. Phys., 114 (2001) 8347. 6

[49] G. M. Harris und J. E. Willard, J. Am. Chem. Soc., 76 (1954) 4678. 9

[50] R. Vogt, R. Sander, R. von Glasow und P. J. Crutzen, J. Atmos. Chem., 32 (1999) 375. 9

[51] K. G. Heumann, Anal. Chim. Act., 283 (1993) 230. 9

[52] H. E. Simmons und R. D. Smith, J. Am. Chem. Soc., 81 (1959) 4256. 9, 21

[53] D. C. Blomstrom, K. Herbig und H. E. Simmons, J. Org. Chem., 30 (1965) 959. 9

[54] O. Hassel und H. Viervoll, Acta Chem. Scand., 1 (1947) 149. 9

[55] M. Ito, P.-K. C. Huang und E. M. Kosower, Trans. Faraday Soc., 57 (1961) 1662. $9,10,14,50$ 
[56] P. M. Kroger, P. C. Demou und S. J. Riley, J. Chem. Phys., 65 (1976) 1823. 9, $10,12,90,92$

[57] F. L. Voelz, F. F. Cleveland und A. G. Meister, J. Opt. Soc. Am., 43 (1953) 1061. 10

[58] F. Duscheck, M. Schmitt, P. Vogt, A. Materny und W. Kiefer, J. Raman Spec., 28 (1997) 445. 10, 14, 137

[59] Z. Kisiel, L. Pszczółkowski, W. Caminati und P. G. Favero, J. Chem. Phys., 105 (1996) 1778. 10, 99

[60] J. Zhang, E. J. Heller, D. Huber und D. G. Imre, J. Chem. Phys., 89 (1988) 3602. 10, 13

[61] J. Zhang und D. G. Imre, J. Chem. Phys., 89 (1988) 309. 10, 13, 15, 49, 50, 90

[62] H. Xu, Y. Guo, S. Liu, X. Ma, D. Dai und G. Sha, J. Chem. Phys., 117 (2002) 5722. 10, 12, 14, 49, 50, 51, 92, 95, 133

[63] R. Nolte, A. Seibert und W. Seidel, Z. Naturforsch., 38a (1983) 591. 10

[64] G. Schmitt und F. J. Comes, J. Photochem., 14 (1980) 107. 10, 11, 87

[65] J. B. Koffend und S. R. Leone, Chem. Phys. Lett., 81 (1981) 136. 11, 87

[66] H. Okabe, M. Kawasaki und Y. Tanaka, J. Chem. Phys., 73 (1980) 6162. 11

[67] U. Marvet und M. Dantus, Chem. Phys. Lett., 256 (1996) 57.11

[68] U. Marvet, Q. Zhang, E. J. Brown und M. Dantus, J. Chem. Phys., 109 (1998) 4415. 11

[69] Q. Zhang, U. Marvet und M. Dantus, Faraday Discuss., 108 (1997) 63. 11

[70] I. Pastirk, E. J. Brown, Q. Zhang und M. Dantus, J. Chem. Phys., 108 (1998) 4375. 11

[71] S. L. Baughcum und S. R. Leone, J. Chem. Phys., 72 (1980) 6531. 11, 12, 94, 96

[72] T. F. Hunter und K. S. Kristjansson, Chem. Phys. Lett., 90 (1982) 35. 11

[73] K.-W. Jung, T. S. Ahmadi und M. A. El-Sayed, Bull. Korean Chem. Soc., 18 (1997) 1274. 11, 90

[74] J. Sehested, T. Ellermann und O. J. Nielsen, Int. J. Chem. Kinet., 26 (1994) 259. 12, 95, 133

[75] Ğ. Barinovs, N. Marković und G. Nyman, J. Chem. Phys., 111 (1999) 6705. 14, 90 
[76] D. Senapati, K. Kavita und P. K. Das, J. Phys. Chem. A, 106 (2002) 8479. 14

[77] W. M. Kwock und D. L. Phillips, Chem. Phys. Lett., 235 (1995) 260. 14

[78] W. M. Kwock und D. L. Phillips, J. Chem. Phys., 104 (1996) 2529. 14

[79] D. L. Phillips und W. M. Kwock, Chem. Phys. Lett., 241 (1995) 267. 15

[80] W. M. Kwock und D. L. Phillips, J. Chem. Phys., 104 (1996) 9816. 15

[81] W. M. Kwock und D. L. Phillips, Chem. Phys. Lett., 270 (1997) 506. 15

[82] W. M. Kwock und D. L. Phillips, Molec. Phys., 90 (1997) 315. 15

[83] X. Zheng und D. L. Phillips, Chem. Phys. Lett., 313 (1999) 467. 15

[84] J. P. Simons und P. E. R. Tatham, J. Chem. Soc. A, (1966) 854. 15, 16, 17

[85] H. Mohan und P. N. Moorthy, J. Chem. Soc. Perkin Trans. 2, (1990) 277. 17, 18,99

[86] D. Bingemann, A. M. King und F. Flemming Crim, Chem. Phys. Lett., 113 (2000) 5018. 18

[87] C. M. Cheatum, M. M. Heckscher, D. Bingemann und F. Flemming Crim, Chem. Phys. Lett., 115 (2001) 7086. 18, 103

[88] A. Charvat, J. Aßmann, B. Abel, D. Schwarzer, K. Henning, K. Luther und J. Troe, Phys. Chem. Chem. Phys., 3 (2001) 2230. 18, 93, 101, 103, 135

[89] K. Sekiguchi, A. Shimojima und O. Kajimoto, Chem. Phys. Lett., 356 (2002) 84. $18,92,95,96,103$

[90] C. Schroeder, Molekulardynamische Simulationen zum intra- und intermolekularen Schwingungsenergietransfer von ausgewählten Molekülen, Dissertation, Georg-August-Universität Göttingen (2002). 18, 103

[91] C. Heidelbach, D. Schwarzer, J. Schroeder und V. S. Vikhrenko, Chem. Phys. Lett., 291 (1999) 333. 18

[92] V. S. Vikhrenko, C. Heidelbach, D. Schwarzer, V. B. Nemtsov und J. Schroeder, J. Chem. Phys., 110 (1999) 5273. 18

[93] C. Heidelbach, V. S. Vikhrenko, D. Schwarzer und J. Schroeder, J. Chem. Phys., 110 (1999) 5286. 18

[94] M. N. Glukhovtsev und R. D. Bach, Chem. Phys. Lett., 269 (1997) 145. 19, 94

[95] A. E. Orel und O. Kühn, Chem. Phys. Lett., 304 (1999) 285. 19

[96] X. Zheng und D. L. Phillips, J. Phys. Chem. A, 104 (2000) 6880. 20, 21, 95 
[97] X. Zheng, W.-H. Fang und D. L. Phillips, J. Chem. Phys., 113 (2000) 10934. 21

[98] Y.-L. Li, D. Wang und D. L. Phillips, J. Chem. Phys., 117 (2002) 7931. 21

[99] Y.-L. Li, K. H. Leung und D. L. Phillips, J. Phys. Chem. A, 105 (2001) 10621. 21

[100] D. L. Phillips, W.-H. Fang und X. Zheng, J. Am. Chem. Soc., 123 (2001) 4197. 21,94

[101] W.-H. Fang, D. L. Phillips, D.-q. Wang und Y.-L. Li, J. Org. Chem., 67 (2002) 154. 21

[102] D. M. Chen, Y.-L. Li und D. L. Phillips, J. Org. Chem., 67 (2002) 4619. 21

[103] Y.-L. Li, C. Zhao, W. M. Kwock, X. Guan, P. Zuo und D. L. Phillips, J. Chem. Phys., 119 (2003) 4671. 21

[104] J. W. Shelton und J. Armstrong, IEEE J. Quantum Elektron., QE3 (1967) 696. 23

[105] F. Krausz, M. E. Fermann, T. Brabec, P. F. Curley, M. Hofer, M. H. Ober, C. Spielmann, E. Wintner und A. J. Schmidt, IEEE J. Quantum Elektron., 28 (1992) 2097. 23

[106] U. Keller, W. H. Knox und G. W. 'tHooft, IEEE J. Quantum Elektron., 28 (1992) 2123. 23

[107] C. Spielmann, M. Lenzner, A. Stingl, R. Szipöcs und F. Krausz, Phys. Bl., 51 (1995) 289. 23

[108] M. Kling, Experimentelle und Theoretische Untersuchungen der FemtosekundenKinetik des Photoinduzierten Zerfalls Aromatischer Peroxidverbindungen, Dissertation, Georg-August-Universität Göttingen (2002). 24, 41, 42, 56

[109] B. Abel, J. Aßmann, P. Botschwina, M. Buback, M. Kling, R. Oswald, S. Schmatz, J. Schroeder und T. Witte, J. Phys. Chem. A, 107 (2003) 5157. 24

[110] B. Abel, J. Aßmann, M. Buback, C. Grimm, M. Kling, S. Schmatz, J. Schroeder und T. Witte, Ultrafast Decarboxylation of Carbonyloxy Radicals: Influence of Molecular Structure (2003), J. Phys. Chem. A, im Druck. 24

[111] P. Maine, D. Strickland, P. Bado, M. Pessot und G. Mourou, IEEE J. Quantum Elektron., 24 (1988) 398. 24, 25

[112] Clark-MXR Inc., CPA-2001: User's Manual Version 1.0 (1998). 24, 25, 133 
[113] Jobin Yvon GmbH, Manual: NOPA Nichtkollinear gepumptes OPA-System (2001). 24, 29, 133

[114] T. Wilhelm, J. Piel und E. Riedle, Opt. Lett., 22 (1997) 1494. 24, 29, 30

[115] J. Piel, M. Beutter und E. Riedle, Opt. Lett., 25 (2000) 180. 24, 29, 30

[116] Light Conversion, TOPAS: User's Manual (2001). 24, 31

[117] W. Lauterborn und T. Kurz, Coherent Optics, Springer, 2. Aufl. (2003). 25, 28

[118] K. Tamura und M. Nakazawa, Appl. Phys. Lett., 67 (1995) 3691. 25, 26, 133

[119] K. Tamura, E. P. Ippen und H. A. Haus, Appl. Phys. Lett., 67 (1995) 158. 26

[120] O. E. Martinez, J. Opt. Soc. Am. B, 3 (1986) 929. 26

[121] W. Demtröder, Laserspektroskopie, Springer-Verlag, 4. Aufl. (2000). 26

[122] M. K. Reed, M. K. Steiner-Shepard, M. S. Armas und D. K. Negus, J. Opt. Soc. Am. B, 12 (1995) 2229. 29

[123] Z. Bor und B. Ràcz, Opt. Comm., 54 (1985) 165. 30

[124] R. H. Dicke, Phys. Rev., 93 (1954) 99. 31

[125] Hewlett Packard Company, HPVEE, Test and Measurement, Data Acquisition. 34

[126] J. Herrmann und B. Wilhelmi, Laser für ultrakurze Lichtimpulse, Physik Verlag - Weinheim (1984). 35

[127] H. Eichler, U. Klein und D. Langhans, Chem. Phys. Lett., 67 (1979) 21. 35

[128] H. E. Lessing, A. von Jena und M. Reichert, Chem. Phys. Lett., 36 (1975) 517. 36

[129] H. E. Lessing und A. von Jena, Chem. Phys. Lett., 42 (1976) 517. 36

[130] Clark-MXR Inc., DT-505: User's Manual Version 2.4 (1995). 36

[131] R. Mohrschladt, Dynamik von Umlagerungsprozessen über kleine Energiebarrieren in komprimierter Lösung: Tetraphenylethylen und 1,1'-Binaphthyl im $S_{1}$ Zustand, Dissertation, Georg-August-Universität Göttingen (1993). 36, 53

[132] Vielen Dank an Herrn Sauermann für die Konzeption und Herstellung der filigranen Durchflusszellen. 42

[133] Das Prinzip und Design der verwendeten Hochdruckzelle stammt aus der Arbeitsgruppe von Prof. Kajimoto aus Kyoto. 43 
[134] Vielen Dank an dieser Stelle an die Arbeitsgruppe von Prof. Dr. Dirk Schwarzer für die Hilfe und Kooperation beim Bau der Hochdruckzelle. Insbesondere auch Vielen Dank an Dr. Peter Kutne für seine Hilfe in der Testphase der Zelle. 43

[135] P. Kutne, Zeitaufgelöste Untersuchungen zum intramolekularen Schwingungsenergiefluss durch molekulare Ketten, Dissertation, Georg-August-Universität Göttingen (2003). 43

[136] Vielen Dank an Herrn Schmidt für den Bau der Hochdruckzelle. 43

[137] An dieser Stelle meinen Dank an Herrn Dipl. Ing. V. Meier, stellvertretend für die gesamte Mechanikwerkstatt, für die stets hervorragende Arbeit und das Konzipieren aller erdenklichen mechanischen Teile. 43

[138] Vielen Dank an die Elektronikwerkstatt unter Leitung von Dipl. Ing. A. Knorr für die schnelle und kompetente Lösung aller elektronischen Fragen und Probleme. 44

[139] C. W. Lawson, F. Hirayama und S. Lipinsky, J. Chem. Phys., 51 (1969) 1590. 50

[140] National Institute of Standards and Technology, NIST Chemistry WebBook, NIST Standard Reference Database, 69. Aufl. (2003), http://webbook.nist.gov/chemistry/. 54, 63, 72, 104, 126, 127, 130, $131,132,135$

[141] C. L. Thomsen, J. Thøgersen und S. R. Keiding, J. Phys. Chem. A, 102 (1998) 1062. 56

[142] P. Sulzer und K. Wieland, Helv. Phys. Acta, 25 (1951) 653. 59, 95, 101

[143] L. Brouwer und J. Troe, Chem. Phys. Lett., 82 (1981) 1. 59, 101

[144] L. Nikowa, D. Schwarzer und J. Troe, Chem. Phys. Lett., 233 (1995) 303. 59, 101

[145] R. J. Sension, S. T. Repinec, A. Z. Szarka und R. M. Hochstrasser, J. Chem. Phys., 98 (1993) 6291. 59

[146] D. Schwarzer, J. Troe, M. Votsmeier und M. Zerezke, J. Chem. Phys., 105 (1996) 3121. 59, 103

[147] K. Reuter, S. Rosenzweig und E. U. Franck, Physica A, 156 (1989) 294. 72

[148] S. V. Kamat, B. Iwaskewycz, E. J. Beckman und A. J. Russell, Proc. Natl. Acad. Sci. USA, 90 (1993) 2940. 72, 107

[149] W. Song, N. Patel und M. Maroncelli, J. Phys. Chem. B, 106 (2002) 8783.72

[150] J. Tellinghuisen, J. Chem. Phys., 58 (1973) 2821. 87 
[151] A. Becker und F. H. M. Faisal, Phys. Rev. A, 59 (1999) R3182. 88

[152] D. Descamps, L. Roos, C. Delfin, A. L'Huillier und C.-G. Wahlström, Phys. Rev. A, 64 (2001) 1. 88

[153] L. M. Chen, J. J. Park, K. H. Hong, I. W. Choi, J. L. Kim, J. Zhang und C. H. Nam, Phys. Plasmas, 9 (2002) 3595. 88

[154] C. Grimm, M. Kling, J. Schroeder, J. Troe und J. Zerbs, Density Dependent Photochemical Branching Ratio in Supercritical $\mathrm{CO}_{2}$ : Photodissociation and Isomerization of Diiodomethane (2003), Israel J. Chem., im Druck. 90, 99

[155] L. Nikowa, D. Schwarzer, J. Troe und J. Schroeder, J. Chem. Phys., 97 (1992) 4827. 90

[156] W. B. DeMore, D. M. Golden, R. F. Hampson, C. J. Howard, C. E. Kolb, M. J. Kurylo, M. J. Molina, A. R. Ravishankara und S. P. Sander, Chemical Kinetics and Photochemical Data for Use in Stratospheric Modeling, Evaluation Number 12, JPL Publication 97-4, NASA National Aeronautics and Space Administration / Jet Propulsion Laboratory, California Institute of Technology, Pasadena, CA (1997). 92

[157] S. P. Sander, R. R. Friedl, D. M. Golden, M. J. Kurylo, R. E. Huie, V. L. Orkin, G. K. Moortgat, A. R. Ravishankara, C. E. Kolb, M. J. Molina und B. J. Finlayson-Pitts, Chemical Kinetics and Photochemical Data for Use in Atmospheric Studies, Evaluation Number 14, JPL Publication 02-25, NASA National Aeronautics and Space Administration / Jet Propulsion Laboratory, California Institute of Technology, Pasadena, CA (2002), http://jpldataeval.jpl.nasa.gov/. 92

[158] R. M. Whitnell, K. R. Wilson und J. T. Hynes, J. Chem. Phys., 96 (1992) 5354. 96

[159] G. R. Flemming, Chemical Applications of Ultrafast Spectroscopy, Oxford University Press, New York (1986). 96

[160] M. D. Barkley, A. A. Kowalczyk und L. Brand, J. Chem. Phys., 75 (1981) 3581. 96,98

[161] M. Roy und S. Doraiswamy, J. Chem. Phys., 98 (1993) 3213. 96

[162] G. Seifert und N. Kadarisman, Chem. Phys., 288 (2002) 113. 97

[163] S. Okazaki, M. Matsumoto und I. Okada, J. Chem. Phys., 103 (1995) 8594. 97

[164] G. R. Alms, D. R. Bauer, J. I. Brauman und R. Pecora, J. Chem. Phys., 59 (1973) 5310. 97 
[165] G. R. Alms, D. R. Bauer, J. I. Brauman und R. Pecora, J. Chem. Phys., 59 (1973) 5321. 97

[166] J. L. Dote, D. Kivelson und R. N. Schwartz, J. Phys. Chem., 85 (1981) 2169. 98

[167] J. L. Dote und R. N. Schwartz, J. Phys. Chem., 85 (1981) 3756. 98

[168] G. T. Evans und D. Kivelson, J. Chem. Phys., 84 (1986) 385. 98

[169] J. A. Seetula, Phys. Chem. Chem. Phys., 4 (2002) 455. 99

[170] M. Tamres, R. Foster (Hg.), Molecular Complexes, Paul Elek (Scientific Books) Ltd., London, Bd. 1, 49-116. 99

[171] M. Tamres und R. L. Strong, R. Foster (Hg.), Molecular Associations, Academic Press Inc. (London) Ltd., London, Bd. 2, 331-456. 99, 100

[172] H. Hippler, J. Troe und H. J. Wendelken, J. Chem. Phys., 78 (1983) 5351. 101

[173] M. Quack, Nuovo Cim., 63B (1981) 358. 101

[174] L. Brouwer, H. Hippler, L. Lindemann und J. Troe, J. Phys. Chem., 89 (1985) 4608. 101

[175] K. Sekiguchi, A. Shimojima und O. Kajimoto, Chem. Phys. Lett., 370 (2003) 303. 103

[176] S. G. Kazarian und M. Poliakoff, J. Phys. Chem., 99 (1995) 8624. 107

[177] P. R. Rablen, S. A. Pearlman und D. A. Miller, J. Am. Chem. Soc., 121 (1999) 227. 107

[178] K. Dahl, R. Biwas und M. Maroncelli, J. Phys. Chem. B, 107 (2003) 7838. 107

[179] P. Debye, Polar Molecules, Dover Publications Inc., New York (1929), reprinted. 107

[180] J. E. Lewis, R. Biswas, A. G. Robinson und M. Maroncelli, J. Phys. Chem. B, 105 (2001) 3306. 109, 110, 111, 128, 130

[181] D. Schwarzer, J. Troe und M. Zerezke, J. Chem. Phys., 107 (1997) 8380. 109, 110,111

[182] S. C. Tucker und M. W. Maddox, J. Phys. Chem. B, 102 (1998) 2437. 110, 119

[183] R. Biswas, J. E. Lewis und M. Maroncelli, Chem. Phys. Lett., 310 (1999) 485. 110

[184] W. Song, R. Biwas und M. Maroncelli, J. Phys. Chem. A, 104 (2000) 6924. 110, 111,119 
[185] N. P. Ernsting, S. A. Kovalenko, T. Sensyushkina, J. Saam und V. Farztdinov, J. Phys. Chem. A, 105 (2001) 3443. 116

[186] J. Hahn, Dissertation, Georg-August-Universität Göttingen (2003), in Vorbereitung. 118, 119

[187] K. Oum, Persönliche Mitteilung. 119

[188] R. G. Rubio, J. A. Zollweg, J. M. G. Palanco, J. C. G. Calado, J. Miller und W. B. Streett, J. Chem. Eng. Data, 36 (1991) 171. 128, 129, 135 



\section{Lebenslauf}

Am 11. Juni 1974 wurde ich, Christian Grimm, als Sohn von Hans-Georg Grimm und dessen Ehefrau Ursula Grimm, geb. Waldheim, in Göttingen geboren.

Von 1980 bis 1984 besuchte ich die Hagenberg-Grundschule in Göttingen. Im August 1984 wechselte ich auf die Berthold-Brecht-Orientierungsstufenschule in Göttingen. Von 1987 bis 1993 besuchte ich das Hainberg-Gymnasium in Göttingen, an dem ich am 12. Mai 1993 mit dem Abitur das Zeugnis der allgemeinen Hochschulreife erhielt.

Von Juli 1993 bis Juni 1994 leistete ich meinen Wehrdienst bei der 4. FlaRakGrp 25 in Lohne/Ahlhorn.

Zum Wintersemester 1994/1995 immatrikulierte ich mich an der Georg-AugustUniversität Göttingen für den Diplom-Studiengang Physik und legte dort am 18. Oktober 1996 die Diplomvorprüfung ab. Im Oktober 1998 begann ich mit meiner Diplomarbeit am Institut für Physikalische Chemie unter der Leitung von Prof. J. Troe mit dem Thema „Untersuchungen der Fluoreszenzlebensdauern von Benzocyclobutenylidenbenzocyclobuten in der Gasphase“, welche ich im Mai 2000 mit der Diplomprüfung abschloss.

Anschließend beschäftigte ich mich als wissenschaftlicher Mitarbeiter im Teilprojekt B7 des Sonderforschungsbereichs 357 „Molekulare Mechanismen unimolekularer Prozesse" am Institut für Physikalische Chemie in Göttingen mit der Femtosekundenspektroskopie zur Untersuchung der Beeinflussung unimolekularer Reaktionen durch die Umgebung und insbesondere mit der Photodissoziation und den Käfigprozessen kleiner Moleküle. Als Ergebnis entstand die vorliegende Dissertation. 